\title{
Advanced insights in upper limb function of individuals with cervical spinal cord injury
}

Inge-Marie Velstra

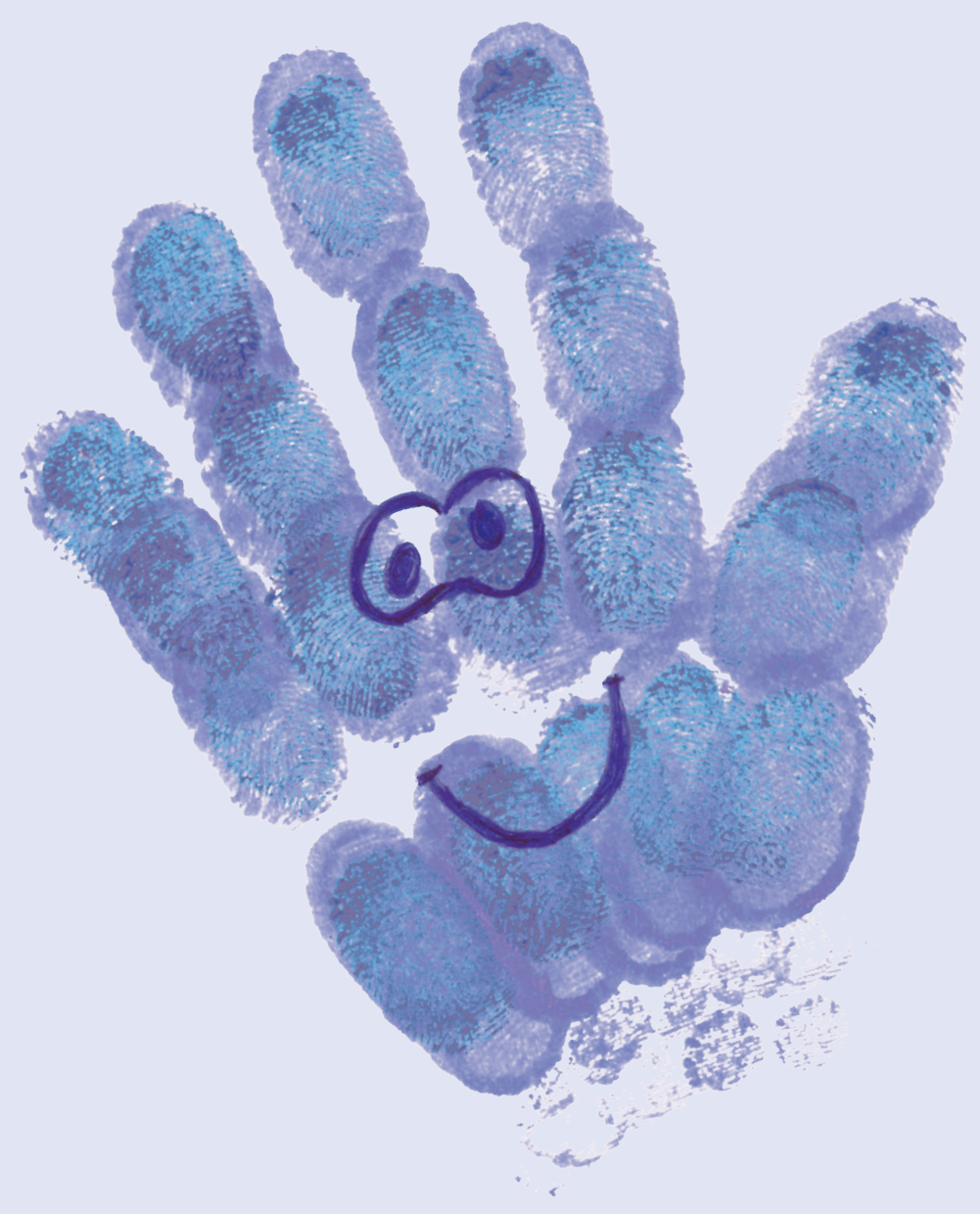



ADVANCED INSIGHTS IN UPPER LIMB FUNCTION OF INDIVIDUALS WITH CERVICAL SPINAL CORD INJURY

INGE-MARIE VELSTRA 
Address of correspondence

Inge-Marie Velstra

Schweizer Paraplegiker-Zentrum

Clinical Trial Unit

Guido A. Zäch Strasse 1

6207 Nottwil

Switzerland

inge-marie.velstra@paraplegie.ch

Cover Quirine Velstra

Layout Renate Siebes, Proefschrift.nu

Printed by Ridderprint, Ridderkerk

ISBN 978-90-365-3929-6

DOI $\quad 10.3990 / 1.9789036539296$

\section{(C) 2015 copyright: I.-M. Velstra}

All right reserved. No part of this publication may be reproduced, stored in a retrieval system, or transmitted, in any means, electronic, photocopying, or otherwise, without permission of the author. The copyright of the articles that have been published, has been referred to the respective journals. 


\title{
ADVANCED INSIGHTS IN UPPER LIMB FUNCTION OF INDIVIDUALS WITH CERVICAL SPINAL CORD INJURY
}

\section{DISSERTATION}

\author{
to obtain \\ the degree of doctor at the University of Twente, \\ on the authority of the rector magnificus, \\ Prof.dr. H. Brinksma, \\ on account of the decision of the graduation committee, \\ to be publicly defended \\ on Friday 23 October 2015 at 12:45
}

by

\section{INGE-MARIE VELSTRA}

born on 1 May 1967

at Apeldoorn 
The supervisors and the co-supervisor have approved the PhD thesis/technical design.

\section{Supervisors}

Prof.dr. J.S. Rietman

Prof.dr. A. Curt

\section{Co-supervisor}

Dr. M. Bolliger 


\section{Graduation committee}

Chairman:

Supervisors:

Co-supervisor:

Members:
Prof.dr. D.G.M.R. Dewulf

Prof.dr. J.S. Rietman

Prof.dr. A. Curt

Dr. M. Bolliger

Prof.dr. V. Dietz

Prof.dr. M.W.M. Post

Prof.dr.ir. H.F.J.M. Koopman

Prof.dr.ir. H.J. Hermens 
The work presented in this thesis was carried out at the Clinical Trial Unit Nottwil and supported by the Swiss Paraplegic Centre Nottwil.

This thesis was printed with the financial support of the Swiss Paraplegic Centre Nottwil, Roessingh Research and Development, and Orthopartner. 


\section{Contents}

$\begin{array}{lll}\text { Chapter } 1 & \text { Introduction } & 9\end{array}$

Chapter 2 A systematic literature review of outcome measures for upper $\quad 21$ extremity function using the international classification of functioning, disability, and health as reference

Chapter 3 Changes in strength, sensation and prehension in acute cervical 57 spinal cord injury: European multicenter responsiveness study of the Graded Redefined Assessment of Strength, Sensibility and Prehension (GRASSP)

Chapter 4 Epicritic sensation in cervical spinal cord injury: diagnostic gains 85 beyond testing light touch

Chapter 5 Prediction and stratification of upper limb function and self103 care in acute cervical spinal cord injury (SCI) with the Graded Redefined Assessment of Strength, Sensibility and Prehension (GRASSP)

Chapter 6 Predictive value of upper limb muscles and grasp patterns on functional outcome in cervical spinal cord injury

Chapter 7 General discussion

Samenvatting

Danksagungen / Acknowledgements / Dankwoord

Curriculum vitae

RRD Progress-range 

Chapter 1

\section{Introduction}




\section{Spinal cord injury (SCl)}

A spinal cord injury ( $\mathrm{SCl}$ ) is a damage or trauma to any part of the spinal cord. This often causes permanent changes in strength, sensation, autonomic functions and other body functions below the level of the injury. The extent of SCl depends on the level and completeness of the lesion. The lesion level is classified using the American Spinal Injury Association's (ASIA) Impairment Scale (AIS) classification according to the International Standards for Neurological Classification of Spinal Cord Injury (ISNCSCI) ${ }^{1}$ which focusses on key muscles and key sensory points. The sensory and motor level are defined as the most caudal spinal levels demonstrating normal sensation for both pin prick (PP) and light touch (LT) and normal key muscle group strength. The neurological level of injury is the most caudal level at which both motor and sensory level are intact. The level of completeness is defined using the AIS. The ISNCSCI differentiates between AIS A, B, $\mathrm{C}, \mathrm{D}$ and $\mathrm{E}$. A complete $\mathrm{SCl}$ implies that there is no function below the level of injury: no sensation and no voluntary movement. Individuals with AIS A or B are categorized as having motor complete lesions and individuals with an AIS C or D are classified as having motor incomplete lesions. Spinal cord injury results in tetraplegia when the lesion is at or above the level of T1 and affects arms, hands, trunk, legs and pelvic organs. Paraplegia is caused by lesions below T1 and the trunk, legs and pelvic organs are affected but the upper limb is intact. Besides the neurological dysfunctions, many secondary problems like urinary tract infections, pulmonary complications, blood pressure disturbances, sexual dysfunction, spasticity or pain may develop. The combination of neurological dysfunction and secondary problems in general results in reduced functioning in activities of daily living (ADLs) and affects an individual's independence, participation and quality of life.

\section{Epidemiology}

Injuries to the spinal cord may either be traumatic (due to motor vehicle accidents, falls or sport/leisure/work related accidents) or non-traumatic (due to spinal canal stenosis, tumors, infections, myelitis or ischemia). In general, traumatic SCls affect more commonly males than females, although large variations exist concerning the male-female ratio between different countries. ${ }^{2}$ The mean age at injury for traumatic lesions lies between 20 and 40 in the majority of cases. ${ }^{2-4}$ This indicates that individuals with traumatic $\mathrm{SCl}$ are relatively young when the injury occurs and thus they need specific care for prolonged periods of time which generates larger lifetime costs than other diseases such as stroke. In contrast, a growing number of elderly in traumatic SCI was observed in the Netherlands in $2010 .^{5}$ 
Furthermore in a recent publication, ${ }^{6}$ three age peaks were noticed in acute traumatic and non-traumatic SCl in Switzerland (e.g. 30-40, 45-55 and 60-75 years).

Nijendijk et al. ${ }^{5}$ reported that the incidence rate of traumatic $\mathrm{SCl}$ in the Netherlands in 2010 was around 11.7 per million population per year. The crude annual incidence rate of traumatic SCI in Switzerland between 2005-2011 was estimated at 18 per million population. ${ }^{7}$ The worldwide annual incidence of traumatic $\mathrm{SCl}$ ranged from 8 to 49 persons per million population. ${ }^{2}$ Although incidence rates vary widely across countries, the incidence of $\mathrm{SCl}$ is relatively low compared with other conditions, which is a challenge for the recruitment of suitable participants for clinical studies.

\section{Evaluation of upper limb function in cervical SCI}

The upper limbs play an essential role in people's lifes, because they are fundamental for performing ADLs such as self-care, various types of work, leisure and social activities. Individuals with cervical SCI suffer from motor and sensory impairments which cause limited upper limb function and effect the performance of ADLs. ${ }^{8}$ This ultimately leads to impaired independence ${ }^{9}$ and restricted participation as well as decreased quality of life. Indeed, previous studies have shown that individuals with tetraplegia consider improvements in upper limb function to be one of the most significant factors in improving their quality of life. ${ }^{10-12}$ Several upper limb outcome measures are available. However, only a few have been specifically developed for $\mathrm{SCl}$ and these have limited psychometric properties. ${ }^{13-15}$ Therefore, there is a clear need for valid, reliable and responsive outcome measures in cervical $\mathrm{SCl}$, in order to assess upper limb function accurately.

The ISNCSCI ${ }^{1}$ is the current standard for evaluating the neurologic status and the recovery after $\mathrm{SCI}$ and includes the AIS. The AIS classifies individuals with $\mathrm{SCI}$ in wideranging categories and comprises a highly heterogeneous population in terms of level and severity of the injury with respect to the whole body. ${ }^{16}$ Thus, the ISNCSCI was not designed to be specific to the upper limb function which undermines the effectiveness of the AIS in the assessment of upper limb neurological recovery.

The Spinal Cord Independence Measure (SCIM) is the most widely used outcome measure to assess independence in fundamental daily activities and is useful to document changes in ADLs in individuals with $\mathrm{SCl} .{ }^{17}$ The utilization of a global outcome measure such as the SCIM, although providing clinically meaningful categorization of functioning in ADLs, does not provide insights into the underlying sensorimotor function driving functional recovery. Accordingly, the SCIM is not suitable to discern functional improvement arising 
from actual healing of damaged spinal cord tissue versus rehabilitation training, mood factors and whether the performed tasks are performed bimanually or with compensatory movements, given the SCIM's focus on gained independence. ${ }^{17}$

Likewise, assessments such as the Tetraplegia Hand Activity Measure (THAQ), ${ }^{18}$ the Van Lieshout test (VLT) ${ }^{19,20}$ and the Capability of Upper Extremity Test (CUE) $)^{21,22}$ provide important information regarding the overall arm and hand usage. They are not designed to provide detailed and reliable information about changes in specific sensory and motor impairments affecting upper limb function. Also assessments like the Grasp Release Test $(\mathrm{GRT})^{23}$ and the Motor Capacity Scale(MCS) ${ }^{24,25}$ are specifically designed to assess the effect of neuroprosthetic interventions or upper limb surgery and have not been adopted universally. Furthermore, they do not provide information how changes in impairment contribute to complex upper limb functional tasks.

Moreover, as a result of a wide range of recovery ${ }^{26,27}$ after cervical SCI and the state of upper limb restoration research in $\mathrm{SCl}$, it is of paramount importance to evaluate upper limb function in cervical SCI comprehensively. The widely used International Classification of Functioning, Disability and Health $(\mathrm{ICF})^{28}$ provides a useful framework to improve the appreciation of clinical recovery.

Considering the aforementioned limitations, the Graded Redefined Assessment of Strength, Sensibility and Prehension (GRASSP) was developed as a clinical outcome measure specific to upper limb function in cervical SCI. The items of the GRASSP were generated from existing tests and measures which were reviewed by researchers and clinicians to determine suitability. The initial GRASSP ${ }^{29}$ combined parts of the preexisting Link Hand Function Test ${ }^{30}$ (LiHFT) which is a modification of the Sollerman Hand Function Test $^{31}$ (SHFT), the Tetraplegia Hand Measure (THM) and sensory testing instruments for peripheral hand injury. ${ }^{32}$ It incorporated three domains: strength, sensibility and prehension, which is the basis for the name of the measure. GRASSP - strength is assessed using manual muscle testing (MMT) of 10 muscles of both upper limbs ( 3 in the arm, 7 in the hand). GRASSP - sensibility is assessed with the pocket version of the Semmes and Weinstein monofilament (SWM) at 3 dorsal and palmar sensory test locations of each hand. GRASSP - qualitative grasping (QIG) assesses three predefined grasp forms (cylindrical grasp, lateral key pinch, and tip-to-tip pinch) in both hands and does not require the ability to actually grasp an object. Quantitative grasping (QtG) assesses 6 prehension tasks (e.g. grasping or moving a coin) in a standardised way for each arm separately. Each domain can be tested individually or in conjunction with another domain. Therefore, the GRASSP 
covers different aspects of upper limb function for evaluating changes within the motor and sensory systems. Furthermore, it reflects impairment changes that fall into the ICF ${ }^{28}$ component "body structure and body function". These changes contribute to complex upper limb tasks, which refer to the ICF component "activity and participation".

In individuals with chronic cervical SCI (i.e. more than 6 months post injury), the GRASSP has shown high validity and excellent overall inter- and intra-rater reliability. ${ }^{33} \mathrm{An}$ important criterion for a clinical outcome measure, such as the GRASSP, is its sensitivity to detect changes in upper limb function over time..$^{34-37}$ This facilitates the evaluation of recovery patterns and treatment efficacy of experimental interventions in cervical SCI. The use of the GRASSP is recommended in the very early acute phases after injury to approximately one year post injury. However, responsiveness has not yet been investigated and is therefore one of the aims within this thesis. In general, little has been published on prediction of functional outcome following $\mathrm{SCl},{ }^{38-40}$ and in particular, data on prediction and stratification of upper limb function and self-care after incomplete cervical SCl is lacking. ${ }^{41}$

\section{Aims}

The overall aim of this PhD thesis is therefore to study the assessment, evaluation and prediction of upper limb function up to one year post injury using the GRASSP in individuals with cervical SCI.

The specific aims of this thesis are:

a. To provide information regarding the (1) responsiveness and reliability of different outcome measures used with persons who have impairments in upper extremity function and (2) their content validity based on the International Classification of Functioning, Disability, and Health (ICF).

b. To investigate the internal and external responsiveness and recovery profiles of the GRASSP instrument in revealing changes in upper limb function within the first year following cervical SCI.

c. To compare the epicritic sensation assessed by Ligth Touch (LT), SemmesWeinstein monofilament (SWM), and electrical perception threshold (EPT) across cervical dermatomes (C3-C8) in individuals with cervical SCI.

d. To evaluate the value of GRASSP in predicting upper limb function and self-care outcomes in individuals with cervical SCI.

e. To determine which single or combined upper limb muscles as defined by the ISNCSCI upper extremity motor score (UEMS) and the GRASSP, 
best predict upper limb function and independence in ADLs, and to assess the predictive value of qualitative grasp movements (QIG) on upper limb function in individuals with acute tetraplegia.

\section{Outline of this thesis}

This thesis contains five papers, which were originally written as separate manuscripts and presented as chapters in logical order. Below, a brief description of the content of these chapters is given.

First, chapter 2 reports the results of a systematic literature review on current outcome measures regarding upper limb function in individuals with (1) peripheral upper extremity conditions, (2) rheumatologic diseases, (3) stroke, and (4) tetraplegia. All outcome measures have been classified according to the ICF. For each outcome measure a description of the concept, operationalisation into variables and instruments as well as psychometric properties was given to determine the availability of preferably objective upper limb function outcome measures within each health condition that are relevant for research and rehabilitation.

Chapter 3 focuses on responsiveness and recovery profiles of the GRASSP in a longitudinal multi-center study. While it was shown that the GRASSP is a valid and reliable measure of upper limb function cross-sectionally, its responsiveness ${ }^{34-37}$ was still unknown. Measures that are valid cross-sectionally are not necessary responsive. ${ }^{42}$ The GRASSP should be able to determine subtle neurological changes in the upper limb and provide information concerning the rehabilitation progress, in order to be able to evaluate the efficacy of rehabilitation and experimental interventions. Therefore, it should be sensitive to detect changes in upper limb function up to 1 year after cervical $\mathrm{SCl}$. In addition, the responsiveness of GRASSP subtests is compared to other clinical outcome measures as well as to a clinician-rated outcome measure (CROM) in order to explore clinical relevance.

Chapter 4 addresses the comparison of epicritic sensation assessed by Light Touch (LT), Semmes-Weinstein monofilament (SWM) and electrical perception threshold (EPT) across cervical dermatomes (C3-C8) in individuals with cervical SCI. The LT assessment of sensation roughly grades the ability of detecting a light touch in the affected dermatome by "absent", "impaired", or "normal". It was not yet known, if the segmental assessment of epicritic sensation in cervical $\mathrm{SCl}$ can be improved by additional semiquantitative sensory measures like the SWM and EPT complementary to LT. These findings are required to evaluate, if LT testing is sensitive enough in interventional studies. 
Chapter $\mathbf{5}$ presents a study on the prediction of upper limb function and self-care following cervical $\mathrm{SCl}$ within 1 year of injury. After cervical $\mathrm{SCl}$, arm and hand function outcomes vary significantly and are not only dependent on the level and completeness of the lesion but also on the degree of recovery, motivation, and performance of the individual. This inherent heterogeneity within individuals following cervical $\mathrm{SCl}^{25,26}$ renders early prediction of upper limb function and self-care challenging. ${ }^{43} \mathrm{~A}$ thorough and adequate clinical assessment of upper limb function in cervical $\mathrm{SCl}$ is important to predict potential functional outcome after rehabilitation. In this longitudinal multi-center cohort study, outcome of upper limb function and self-care measured by subtests of GRASSP and SCIM and predicted by subtests of GRASSP, ISNCSCI and SCIM, is described in individuals with cervical SCI. Chapter 6 describes the influence of individual muscles or muscle groups defined by GRASSP and ISNCSCI on the prediction of upper limb function and ADLs. Furthermore, the effect of specific grasp patterns, described in the GRASSP, on the prediction of upper limb function in individuals with acute tetraplegia is identified.

The last chapter, chapter 7 , provides a general discussion and reflects on the findings from the various studies. Conclusions are translated into clinical implications and methodological considerations as well as recommendations for future research are formulated.

\section{References}

1. Kirshblum SC, Waring W, Biering-Sorensen F, et al. Reference for the 2011 revision of the International Standards for Neurological Classification of Spinal Cord Injury. J Spinal Cord Med 2011;34:547-554.

2. Singh A, Tetreault L, Kalsi-Ryan S, Nouri A, Fehlings MG. Global prevalence and incidence of traumatic spinal cord injury. Clinical epidemiology 2014;6:309-331.

3. van den Berg ME, Castellote JM, Mahillo-Fernandez I, de Pedro-Cuesta J. Incidence of spinal cord injury worldwide: a systematic review. Neuroepidemiology 2010;34:184-192; discussion 192.

4. Wyndaele M, Wyndaele JJ. Incidence, prevalence and epidemiology of spinal cord injury: what learns a worldwide literature survey? Spinal Cord 2006;44:523-529.

5. Nijendijk JH, Post MW, van Asbeck FW. Epidemiology of traumatic spinal cord injuries in The Netherlands in 2010. Spinal Cord 2014;52:258-263.

6. Krebs J, Katrin Brust A, Tesini S, et al. Study participation rate of patients with acute spinal cord injury early during rehabilitation. Spinal Cord 2015.

7. Meier S, for the SwiSCl study group. Epidemiology of Traumatic Spinal Cord Injury in Switzerland: etiology and rehabilitation outcomes. Poster at ISCos congress. Istanbul, 2013. 
8. Burns AS, Ditunno JF. Establishing prognosis and maximizing functional outcomes after spinal cord injury: a review of current and future directions in rehabilitation management. Spine (Phila Pa 1976) 2001;26:S137-145.

9. Lysack CL, Zafonte CA, Neufeld SW, Dijkers MP. Self-care independence after spinal cord injury: patient and therapist expectations and real life performance. J Spinal Cord Med 2001;24:257265.

10. Anderson KD. Targeting recovery: priorities of the spinal cord-injured population. J Neurotrauma 2004;21:1371-1383.

11. Snoek GJ, MJ IJ, Hermens HJ, Maxwell D, Biering-Sorensen F. Survey of the needs of patients with spinal cord injury: impact and priority for improvement in hand function in tetraplegics. Spinal Cord 2004;42:526-532.

12. Hanson RW, Franklin MR. Sexual loss in relation to other functional losses for spinal cord injured males. Arch Phys Med Rehabil 1976;57:291-293.

13. Dunn JA, Sinnott KA, Bryden AM, Connolly SJ, Rothwell AG. Measurement issues related to upper limb interventions in persons who have tetraplegia. Hand Clin 2008;24:161-168.

14. van Tuijl JH, Janssen-Potten YJ, Seelen HA. Evaluation of upper extremity motor function tests in tetraplegics. Spinal Cord 2002;40:51-64.

15. Velstra IM, Ballert CS, Cieza A. A systematic literature review of outcome measures for upper extremity function using the international classification of functioning, disability, and health as reference. PM R 2011;3:846-860.

16. Fawcett JW, Curt A, Steeves JD, et al. Guidelines for the conduct of clinical trials for spinal cord injury as developed by the ICCP panel: spontaneous recovery after spinal cord injury and statistical power needed for therapeutic clinical trials. Spinal Cord 2007;45:190-205.

17. Catz A, Itzkovich M, Agranov E, Ring H, Tamir A. SCIM--spinal cord independence measure: a new disability scale for patients with spinal cord lesions. Spinal Cord 1997;35:850-856.

18. Land NE, Odding E, Duivenvoorden HJ, Bergen MP, Stam HJ. Tetraplegia Hand Activity Questionnaire (THAQ): the development, assessment of arm-hand function-related activities in tetraplegic patients with a spinal cord injury. Spinal Cord 2004;42:294-301.

19. Post MW, Van Lieshout G, Seelen HA, Snoek GJ, ljzerman MJ, Pons C. Measurement properties of the short version of the Van Lieshout test for arm/hand function of persons with tetraplegia after spinal cord injury. Spinal Cord 2006;44:763-771.

20. Spooren Al, Janssen-Potten YJ, Post MW, Kerckhofs E, Nene A, Seelen HA. Measuring change in arm hand skilled performance in persons with a cervical spinal cord injury: responsiveness of the Van Lieshout Test. Spinal Cord 2006;44:772-779.

21. Marino RJ, Patrick M, Albright W, et al. Development of an objective test of upper-limb function in tetraplegia: the capabilities of upper extremity test. Am J Phys Med Rehabil 2012;91:478486.

22. Marino RJ, Shea JA, Stineman MG. The Capabilities of Upper Extremity instrument: reliability and validity of a measure of functional limitation in tetraplegia. Arch Phys Med Rehabil 1998;79:1512-1521.

23. Wuolle KS, Van Doren CL, Thrope GB, Keith MW, Peckham PH. Development of a quantitative hand grasp and release test for patients with tetraplegia using a hand neuroprosthesis. J Hand Surg Am 1994;19:209-218. 
24. Fattal C, Thery JM, Micallef JP. [Validation of the motor capacities scale: a specific evaluation of manual abilities in tetraplegics who undergo functional surgery of the upper limbs]. Annales de readaptation et de medecine physique : revue scientifique de la Societe francaise de reeducation fonctionnelle de readaptation et de medecine physique 2004;47:537-545.

25. Fattal C. Motor capacities of upper limbs in tetraplegics: a new scale for the assessment of the results of functional surgery on upper limbs. Spinal Cord 2004;42:80-90.

26. Steeves JD, Kramer JK, Fawcett JW, et al. Extent of spontaneous motor recovery after traumatic cervical sensorimotor complete spinal cord injury. Spinal Cord 2011;49:257-265.

27. Kramer JL, Lammertse DP, Schubert M, Curt A, Steeves JD. Relationship between motor recovery and independence after sensorimotor-complete cervical spinal cord injury. Neurorehabil Neural Repair 2012;26:1064-1071.

28. Organization WH. International Classification of Functioning, Disability and Health (ICF) [online]. Available at: http://www.who.int/.

29. Kalsi-Ryan S, CurtA, Fehlings MG, Verrier MC. Assessment of the Hand in Tetraplegia Using the Graded Redefined Assessment of Strength, Sensibility and Prehension (GRASSP): Impairment Versus Function. Top Spinal Cord Inj Rehabil 2009;14:34-46.

30. Rudhe C. The Link Hand Function Test for Patients with a Cervical Spinal Cord Injury: An Intra-Rater and Inter-Rater Reliability and Expert Opinion Evaluation Study. unpublished work, 2004.

31. Sollerman C, Ejeskar A. Sollerman hand function test. A standardised method and its use in tetraplegic patients. Scandinavian journal of plastic and reconstructive surgery and hand surgery / Nordisk plastikkirurgisk forening [and] Nordisk klubb for handkirurgi 1995;29:167-176.

32. Bell-Krotoski JA. Sensibility testing: current concepts. In: Hunter JM, Mackin EJ, Callahan AD, eds. Rehabilitation of the hand: surgery and therapy. St Louis: CV Mosby, 1995, 129-152.

33. Kalsi-Ryan S, Beaton D, Curt A, et al. The Graded Redefined Assessment of Strength Sensibility and Prehension: reliability and validity. J Neurotrauma 2012;29:905-914.

34. Beaton DE, Bombardier C, Katz JN, et al. Looking for important change/differences in studies of responsiveness. OMERACT MCID Working Group. Outcome Measures in Rheumatology. Minimal Clinically Important Difference. J Rheumatol 2001;28:400-405.

35. Husted JA, Cook RJ, Farewell VT, Gladman DD. Methods for assessing responsiveness: a critical review and recommendations. J Clin Epidemiol 2000;53:459-468.

36. Deyo RA, Diehr P, Patrick DL. Reproducibility and responsiveness of health status measures. Statistics and strategies for evaluation. Controlled clinical trials 1991;12:142S-158S.

37. Deyo RA, Centor RM. Assessing the responsiveness of functional scales to clinical change: an analogy to diagnostic test performance. Journal of chronic diseases 1986;39:897-906.

38. Zorner B, Blanckenhorn WU, Dietz V, Curt A. Clinical algorithm for improved prediction of ambulation and patient stratification after incomplete spinal cord injury. J Neurotrauma 2010;27:241-252.

39. Wilson JR, Grossman RG, Frankowski RF, et al. A clinical prediction model for long-term functional outcome after traumatic spinal cord injury based on acute clinical and imaging factors. J Neurotrauma 2012;29:2263-2271.

40. van Middendorp JJ, Hosman AJ, Donders AR, et al. A clinical prediction rule for ambulation outcomes after traumatic spinal cord injury: a longitudinal cohort study. Lancet 2011;377:10041010. 
41. Steeves JD, Lammertse DP, Kramer JL, et al. Outcome Measures for Acute/Subacute Cervical Sensorimotor Complete (AIS-A) Spinal Cord Injury During a Phase 2 Clinical Trial. Top Spinal Cord Inj Rehabil 2012;18:1-14.

42. Guyatt G, Walter S, Norman G. Measuring change over time: assessing the usefulness of evaluative instruments. Journal of chronic diseases 1987;40:171-178.

43. Schonherr MC, Groothoff JW, Mulder GA, Eisma WH. Prediction of functional outcome after spinal cord injury: a task for the rehabilitation team and the patient. Spinal Cord 2000;38:185191. 


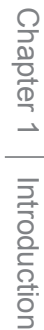



Chapter 2

\section{A systematic literature review of outcome measures for upper extremity function using the international classification of functioning, disability, and health as reference}

Inge-Marie Velstra Carolina S. Ballert Alarcos Cieza 


\section{Abstract}

Objective: To provide information regarding the (1) responsiveness and reliability of different outcome measures used with persons who have impairments in upper extremity function and (2) their content validity based on the International Classification of Functioning, Disability, and Health (ICF).

Data sources: MEDLINE, CINAHL, PsycINFO, and EMBASE databases were systematically searched for studies on outcome measures used to evaluate upper extremity function; only studies written in English and published between July 1997 and July 2010 were considered.

Study selection: One investigator reviewed titles and abstracts of the identified studies to determine whether the studies met predefined eligibility criteria (e.g., study design, age $<18$ years). Another investigator did the same for $70 \%$ of the studies.

Data extraction: All types of outcome measures in the included studies were extracted, and the information retrieved from these outcome measures was linked to the ICF by 2 independent investigators who used standardized linking rules. In addition, studies reporting the clinical responsiveness, interrater reliability, and test-retest reliability of the outcome measures were identified.

Data synthesis: From among the 894 studies that were included in this review, 17 most frequently used outcome measures in the different study populations were identified. Five were patient-reported outcome measures and 12 were clinical outcome measures. The outcome measures show large variability with regard to the areas of functioning and disability addressed. Reliability and responsiveness data are missing for a few outcome measures or for certain populations for which they have been used.

Conclusion: This systematic review provides an overview of the outcome measures used to address functioning and disability as they are related to the upper extremity. The results of this study may help clinicians and researchers select the most appropriate outcome measure for their clinical population or research question according to ICFbased content validity, and additional information on the reliability and responsiveness of the measures is provided. Our findings also can provide directions for further research. 


\section{Introduction}

The upper extremities play an essential role in people's lives because they are integral to performing activities of daily living such as self-care, various types of work, leisure, and social activities. Impairment of the upper extremity can affect other body functions such as sleep or emotional functions ${ }^{1-4}$ and can influence the individual's experience of autonomy and independence..$^{3,5-7}$ Impairment of an upper extremity (e.g., finger amputations or carpal tunnel syndrome) is not only related to peripheral upper extremity conditions but also to other health conditions, such as rheumatologic diseases (e.g., rheumatoid arthritis), stroke, and tetraplegia.

Other investigators report that approximately $70-80 \%$ of persons with stroke have upper extremity impairment ${ }^{8}$ and that more than $75 \%$ of persons with rheumatoid arthritis ${ }^{9}$ show impairments in body functions and limitations in activities associated with upper extremity function. In addition, restoration of upper extremity function is reported to be a major priority for people with tetraplegia. ${ }^{10,11}$

Considering the significant consequences that result from upper extremity impairments, ${ }^{3,4,12-15}$ efforts during the last 2 decades have focused on developing condition-specific outcome measures to assess bodily impairments, activity limitations, and participation restrictions. With the exception of the tetraplegic population, for which few outcome measures specific to upper extremity function have been applied, ${ }^{16}$ a proliferation of outcome measures has been seen in the fields of peripheral upper extremity conditions, ${ }^{17-20}$ rheumatologic disease ${ }^{17,18}$ and stroke. ${ }^{21}$

Thus it is worthwhile to study the areas of functioning, disability, and health that are addressed by different outcome measures that focus on the upper extremity. A comparative examination would allow researchers and health professionals to select the best outcome measure to address the impairments and needs of a specific population in research or practice. The International Classification of Functioning, Disability, and Health (ICF) ${ }^{22}$ is a useful tool for performing such a comparison. ${ }^{23}$ The ICF provides a comprehensive framework for classifying and describing functioning, disability, and health in people with various types of diseases or conditions. The ICF is composed of 4 components-Body Functions, Body Structures, Activities and Participation, and Environmental Factors-that are organized into a hierarchical structure (Figure 2.1). Chapters are related to each component, and each chapter is divided into different levels of categories. ${ }^{23,24}$ For example, the third-level ICF category "d4452 Reaching" is one element of the second-level category "d 445 Hand and Arm Use", which in turn is an element of the chapter "d4 Mobility", which is part of the ICF component "d Activities and Participation". 


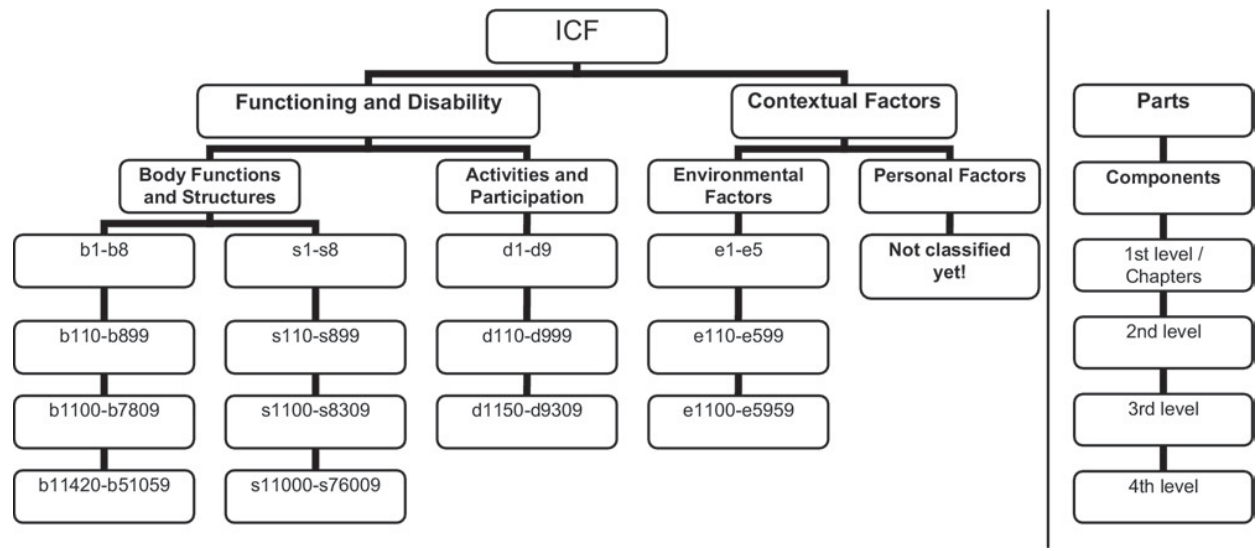

Figure 2.1 The structure of the International Classification of Functioning, Disability and Health (ICF).

An ICF-based comparison also enables the selection of outcome measures that best address the functioning domain in relation to an intervention, which may be at the level of Body Function or Structure, Activities and Participation, or the Environment. Thus the objective of this literature review is to provide an overview of different outcome measures used to address functioning and disability by focusing on persons with impairments in upper extremity function. Our specific aims are to (1) identify outcome measures that address functioning and disability in studies that involve persons with impairments in upper extremity function; (2) compare the content of the identified outcome measures with the ICF as a reference; and (3) report the reliability and responsiveness data of the identified outcome measures when these data are available.

Reliability means "repeatability" or "consistency". An outcome measure can be considered reliable if it provides the same result with repeated applications. Interrater reliability is used to assess the degree to which different raters are consistent on ratings with the same outcome measure. The test-retest reliability is used to find the consistency of a measure over time. Responsiveness describes the ability of an outcome measure to detect clinically important change..$^{25}$ 


\section{Methods}

\section{Study design}

A systematic review encompassing 3 steps was performed. The first step was selection of studies. The second step was identification of outcome measures and extraction of information on the reliability and responsiveness of the extracted outcome measures. The third step was linkage of the information contained within the outcome measures to the corresponding categories of the ICF.

\section{Search strategy and eligibility criteria}

In step 1, the MEDLINE, CINAHL, PsycINFO, and EMBASE databases were used to select interventional and observational studies. We selected studies published from July 1997 to July 2010 and used specific terms related to upper extremity function, such as "hand", "arm", "upper extremity", "function", "activity", "activities", "performance", or "skill". The Boolean operator AND was used to combine these terms with the following terms: "assessment", "measure", "measurement", "instrument", "test”, "evaluation”, "questionnaire", "interview", or "outcome". The following exclusion criteria were used: nonhuman population, language other than English, patient age < 18 years, review or meta-analysis, case report/case series, phase 1 or 2 study, ecological study, economic-evaluation study or decision analysis, comment, letter, editorial, guideline, conference report, book chapter, or dissertation. An initial search was developed for Medline, and this search was then adapted to the other 3 databases.

We checked the abstracts by applying the same general and specific eligibility criteria. Study populations with no impairment in upper extremity function and studies with a sample size $<10$ were excluded. The full text was ordered for selected studies, and the same criteria were used to review the text. Studies that did not provide any reference or information about the psychometric properties of any of the outcome measures used also were excluded.

\section{Data extraction procedure}

In step 2, outcome measure extraction, all types of outcome measures with a reference or information about psychometric properties were extracted. Outcome measures included were categorized according to the study population for which they were used. The following groups were differentiated: (1) peripheral upper extremity conditions, (2) rheumatologic diseases, (3) stroke, and (4) tetraplegia, all of which affect the upper extremity. Because the number of retrieved outcome measures turned out to be very large, the 5 (arbitrary) 
most frequently used outcome measures in each of the study populations were selected for further analysis (Figure 2.2).

In our study the selected outcome measures were categorized into 2 different types of measures: (1) patient-reported outcome measures and (2) clinical outcome measures observed or rated by health professionals. Patient-reported outcome measures contained items reported by the patients or proxy respondents, such as "Are you able to shampoo your hair?" from the Health Assessment Questionnaire (HAQ). ${ }^{26}$ Clinical outcome measures observed or rated by health professionals contained items such as "Wash/dry upper body" from the Quadriplegia Index of Function Scale (QIF), ${ }^{27,28}$ parameters such as "joint range of motion", or tasks such as "Picking up wooden pegs and dropping them in a box" from the Grasp Release Test (GRT). ${ }^{29}$

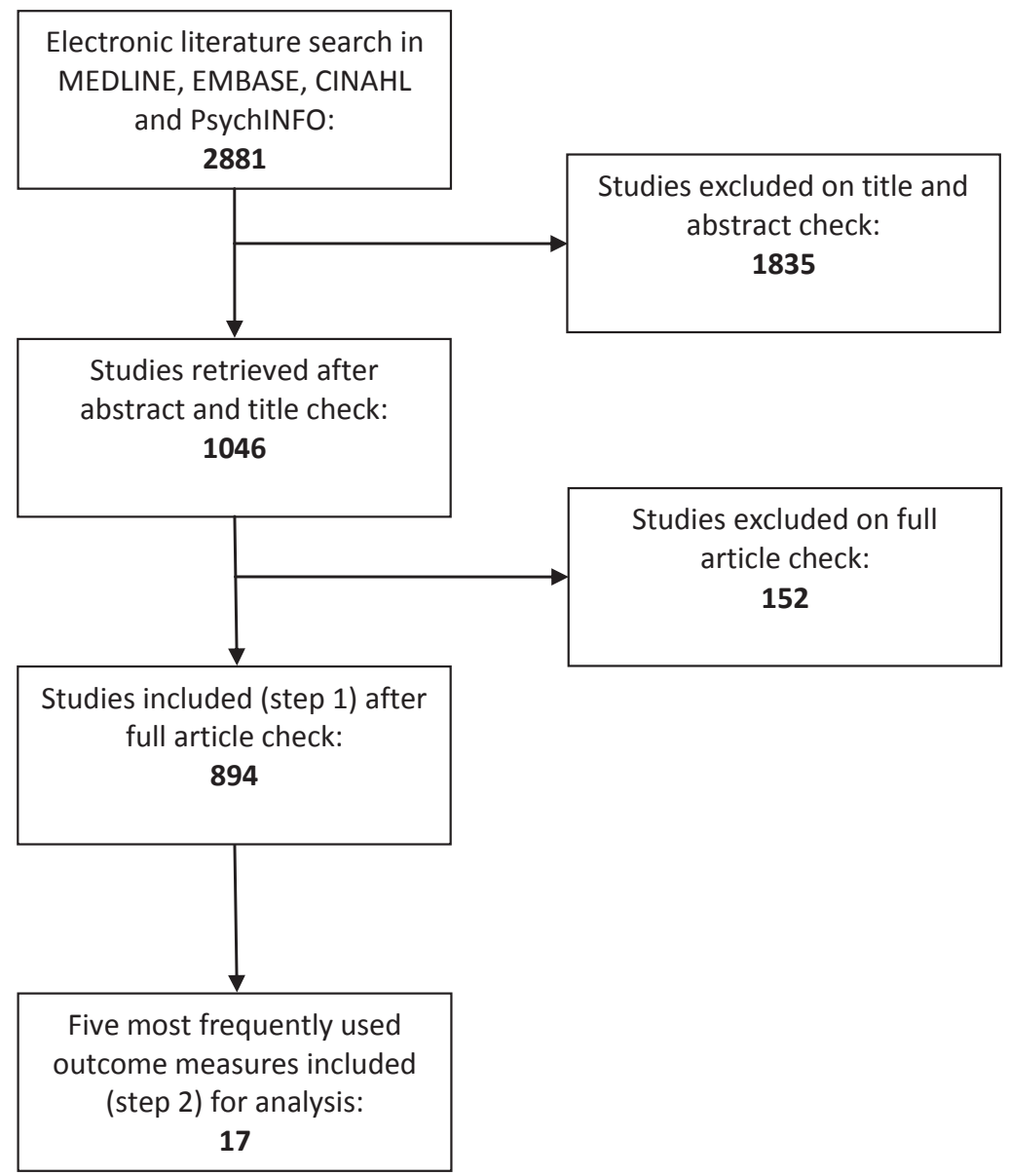

Figure 2.2 Flow chart of the study selection process. 
After having identified the outcome measures, we searched for articles in which investigators examined the reliability and responsiveness of these measures in the populations in which they had been used. The number of patients included in the psychometric study, their health condition, the method applied to study the reliability or responsiveness, the corresponding results, and references were extracted from the identified investigations. Validations of cultural adaptations or translations of the selected outcome measures were not considered in the psychometric properties search. Qualitative studies of the outcome measures of interest performed on subjects with another health condition, from the general population, or with adolescents or children were not included.

\section{Linking to the ICF}

Step 3 was linkage of the information contained in the outcome measures. In this step, each item of the patient-reported outcome measures and each item, aim of each task, or aim of the clinical outcome measure was extracted and linked to the ICF by 2 independent investigators according to a set of linking rules. ${ }^{23,24} \mathrm{An}$ item could be linked to one or more ICF categories, depending on the number of concepts contained in that item. ${ }^{30}$ If specific information could not be linked to the ICF, this was documented and classified in 2 ways. First, if the information was not sufficiently specified to make a decision as to which ICF category or categories should be selected, the option "not definable" was chosen (linking rule 9). For example, the item "My health is excellent" found in the Short Form-36 Health Survey Questionnaire (SF-36) 31,32 $^{2}$ was considered "not definable" for linking. Second, if the information was not represented in the ICF, the option "not covered" was chosen (linking rule 10). For example, the item "How satisfied are you with your HEALTH NOW" of the

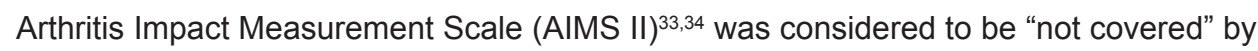
the ICF. If the information referred to a determined diagnosis or disease, "health condition" was used. Personal factors are not yet classified in the ICF. However, when information was considered to address personal factors per definition in the ICF, "personal factor" was used. For example, the item "I seem to get sick a little easier than other people" of the SF-36 was considered a personal factor.

Consensus between the 2 investigators was reached to decide which ICF categories should be linked to the different items or aims. To resolve disagreements between the 2 investigators, a third person trained in the linking rules was consulted. In a discussion led by the third person, the 2 investigators who linked the outcome measures stated their pros and cons for the linking of the information by taking a specific ICF category 
into consideration. The third person made an informed decision on the basis of these statements. The application of the predefined linking rules has been shown to yield high agreement between raters (i.e., $91 \%$ at the second level of the classification). ${ }^{23}$

\section{Quality assurance}

In the abstract checking phase, one investigator reviewed all abstracts for eligibility. Approximately $70 \%$ of abstracts were screened by another investigator. In case of disagreement, the 2 investigators discussed the reasons for inclusion or exclusion of a study. All included articles were examined by one investigator for outcome measure extraction. The linking of the outcome measures selected for further analyses was performed by 2 investigators. Finally, the search of the literature to find the psychometric studies of the selected outcome measures and the corresponding data extraction was performed by one investigator.

\section{Analyses}

Descriptive statistics were used to document the most frequently used outcome measures

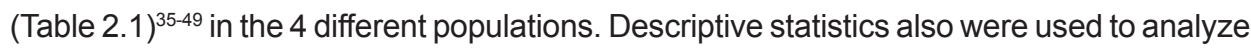
the areas of functioning and disability (i.e., ICF categories) in the most frequently used patient-reported and clinical outcome measures. In addition, the frequency with which an ICF category was being used in the measures was documented.

The degree of agreement between the 2 investigators at the first, second, and third ICF levels was calculated by means of the K statistic and bootstrapped intervals. ${ }^{50,51}$ These analyses were performed with SAS for Windows V 9.1 (SAS Institute, Cary, NC).

\section{Results}

In step 1, the electronic literature searches in MEDLINE, CINAHL, PsycINFO, and EMBASE yielded 2881 hits. One thousand forty-six studies were included after the abstracts were checked for eligibility. The 2 investigators who together checked approximately $70 \%$ of the abstracts $(69.15 \% ; 2881 / 100 * 69.15=1992)$ agreed on 1746 of them $(87.65 \%)$. Of the 2881 studies identified, 894 studies contained a reference or information on the psychometric properties of at least one of the outcome measures used and were definitely included. Figure 2.2 provides an overview of the selection process.

In step 2, the 5 most frequently used outcome measures in each of the study populations were determined, which resulted in a total of 17 outcome measures. 


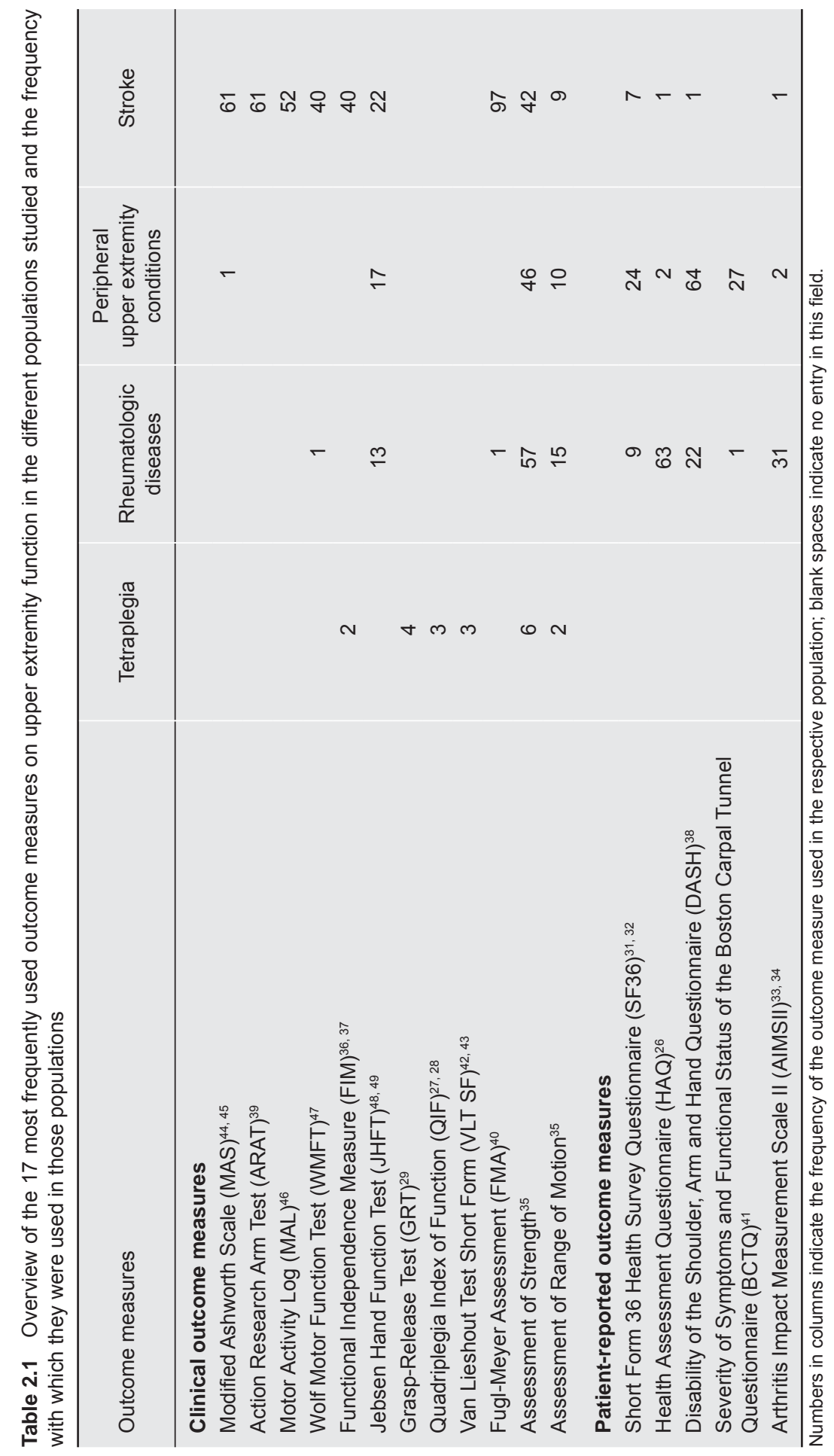


Table 2.1 presents the 17 outcome measures and the frequency with which they were used in the different populations. Five of these outcome measures were patient-reported outcome measures and 12 were clinical outcome measures.

Forty-four publications were identified that included information about the reliability and responsiveness of 15 of the 17 identified outcome measures. Table 2.2 presents the populations for which reliability and responsiveness studies for those outcome measures have been reported, the number of patients included in the studies, the method applied to study the reliability or responsiveness, and the corresponding results and references. ${ }^{52-87}$ For 2 clinical outcome measures (assessment of muscle strength and assessment of joint range of motion), we did not provide information on reliability and responsiveness in Table 2.2. Various devices are available to measure strength (e.g., the dynamometer, the vigorimeter, or the microFET [The Trigenics Institute of Functional Neurology, Toronto, Canada]) and joint range of motion (e.g., the goniometer or the inclinometer), and their assessment corresponds to an objective measurement. The complexity of the resulting psychometric data with different populations and different experimental settings could not be integrated in the analysis of the quality of outcome measures. ${ }^{35}$

The results of step 3 are presented in Tables 2.3-2.5, which show the coverage of ICF categories for the components Body Functions and Structures, Activities and Participation, and Environmental Factors, respectively, by the selected outcome measures. The tables display the linkage results at all levels of the ICF hierarchy, including the frequency with which the ICF categories were covered in the outcome measures. The first column represents the list of ICF categories (first, second, third, and fourth level) addressed in at least one of the measures, whereas the following columns represent the outcome measures. The numbers in each of the columns indicate which of the ICF categories were represented and how often the category was used in the outcome measure.

The outcome measures cover 33 ICF categories of the component Body Function. Eleven outcome measures refer to Chapter b1, Mental Functions; 6 refer to Chapter b2, Sensory Functions and Pain; 3 refer to Chapter b5, Functions of the Digestive, Metabolic, and Endocrine Systems; 1 refers to Chapter b6, Genitourinary and Reproductive Functions; and 12 refer to Chapter b7, Neuromusculoskeletal and Movement-Related Functions.

Two outcome measures cover 8 ICF categories of the component Body Structure, and they belong to Chapter s7, Structures Related to Movement. Fourteen of the 17 outcome measures cover 96 ICF categories of the component Activities and Participation. All ICF chapters of this ICF component are represented. 

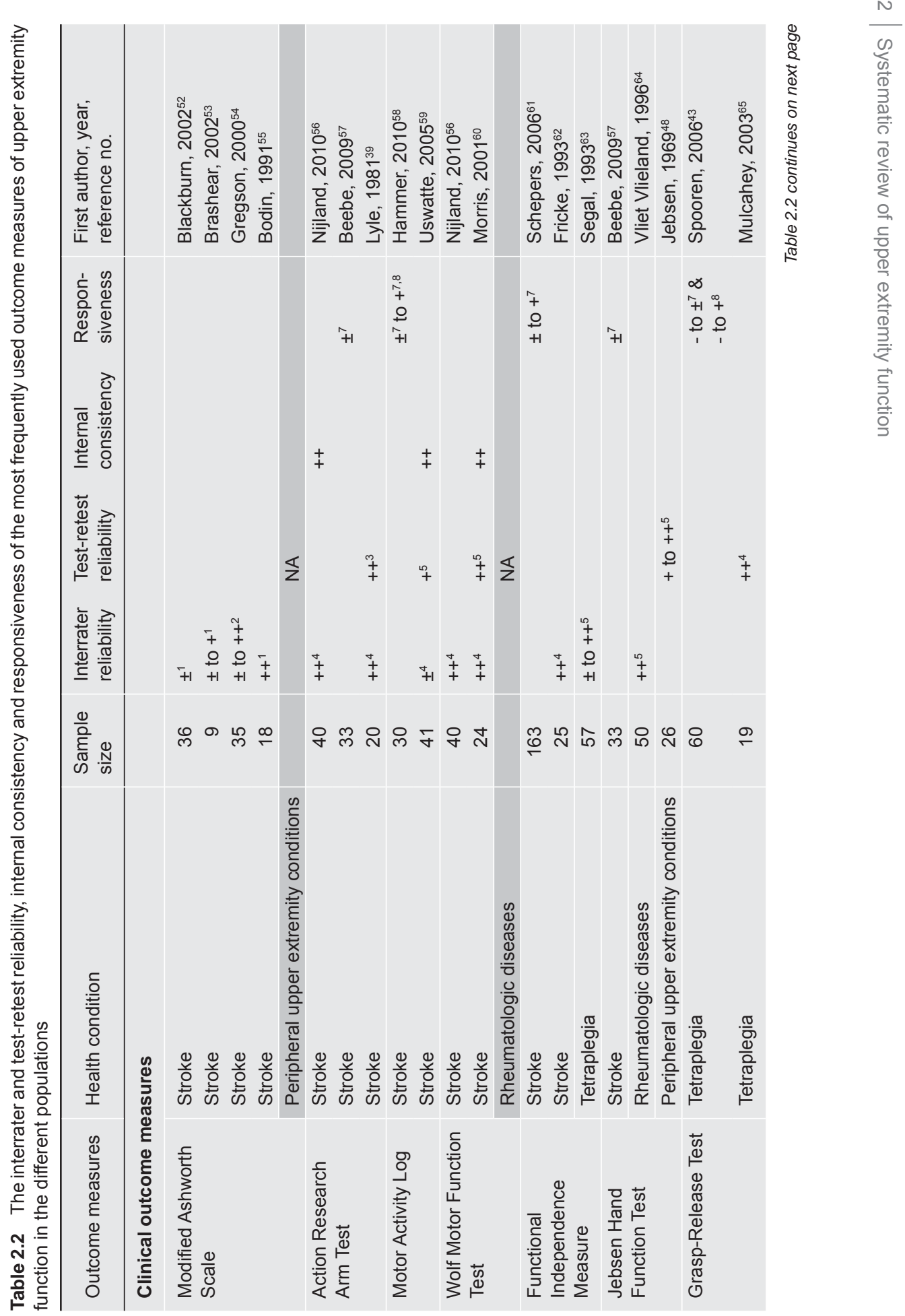


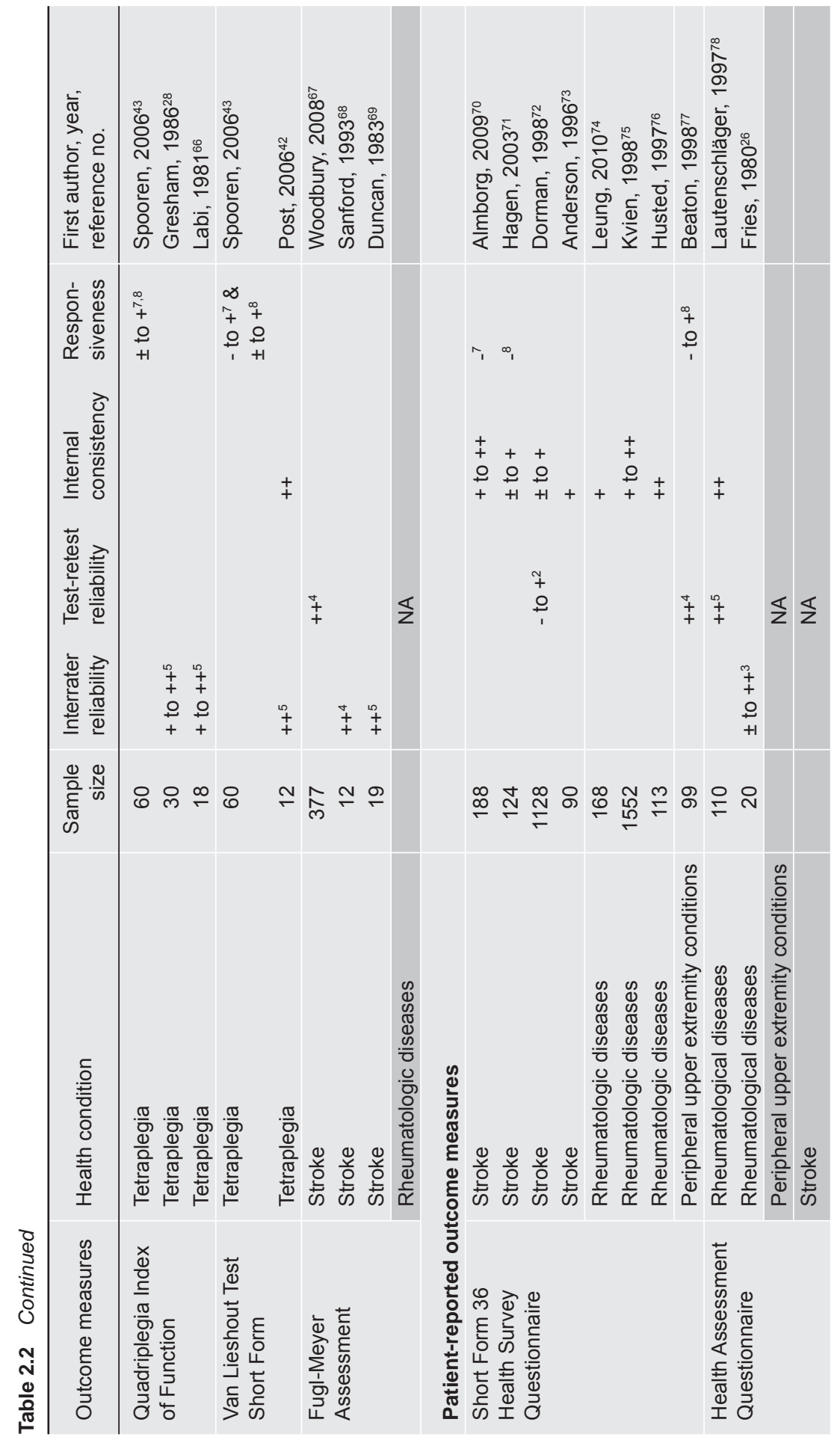




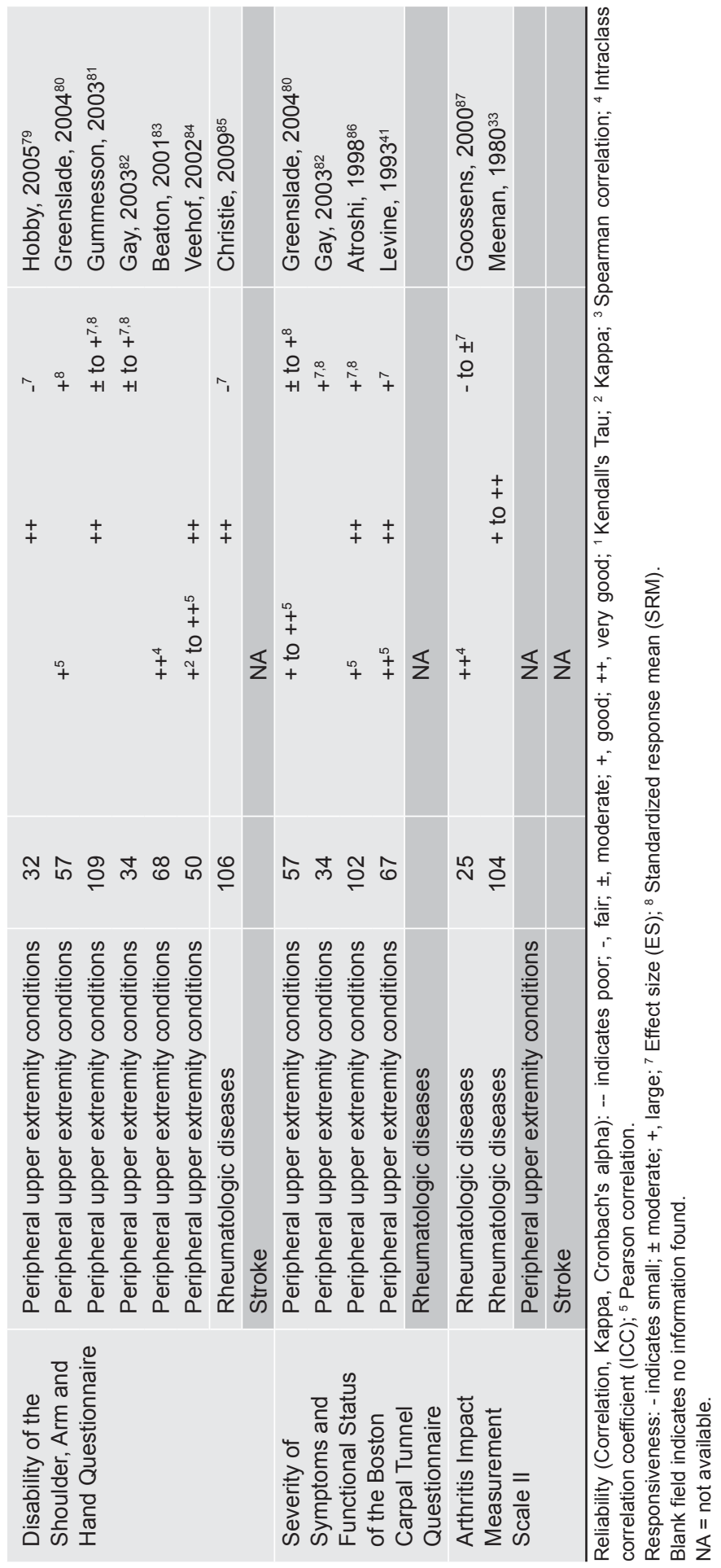




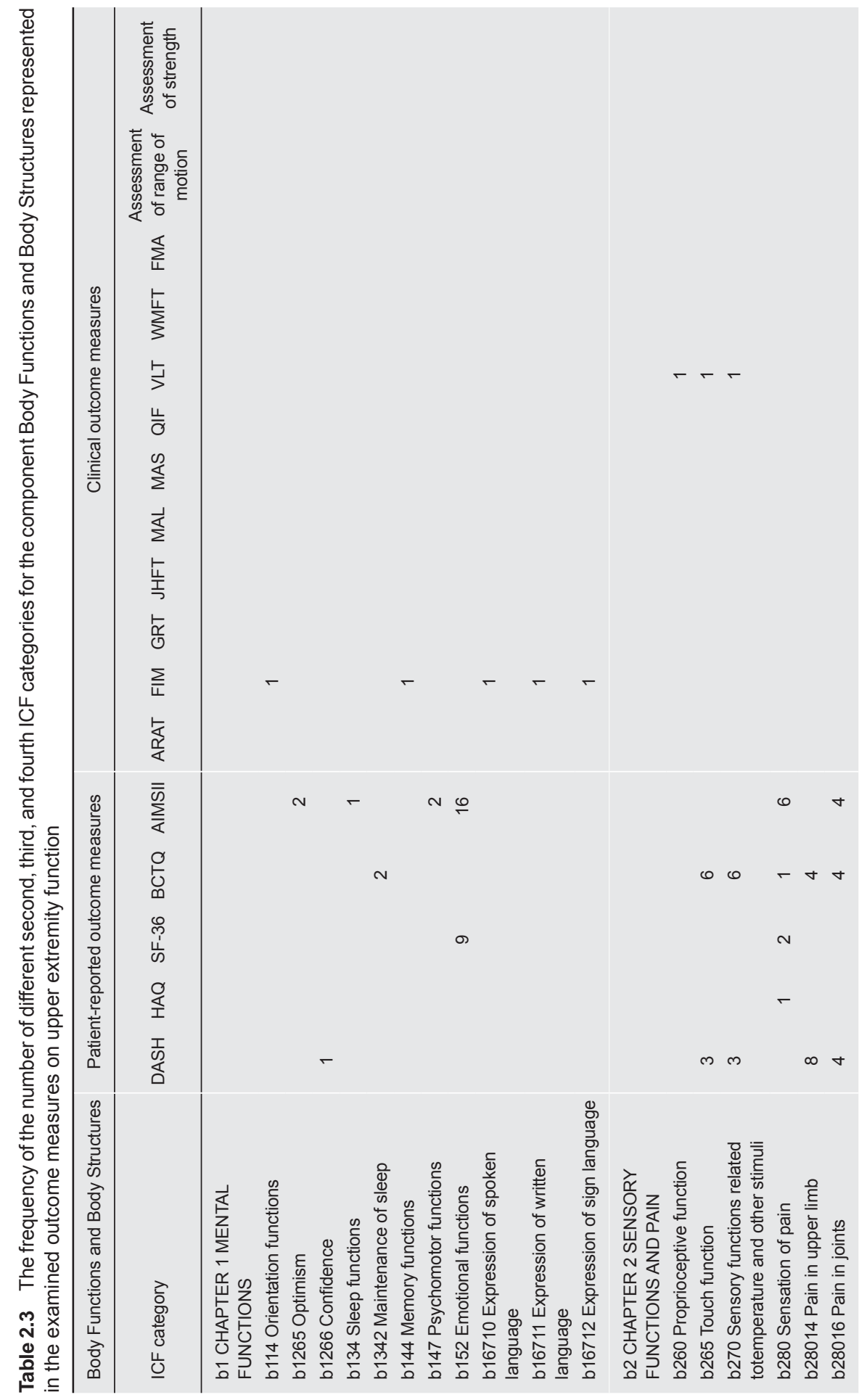


ร

$\simeq$

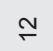






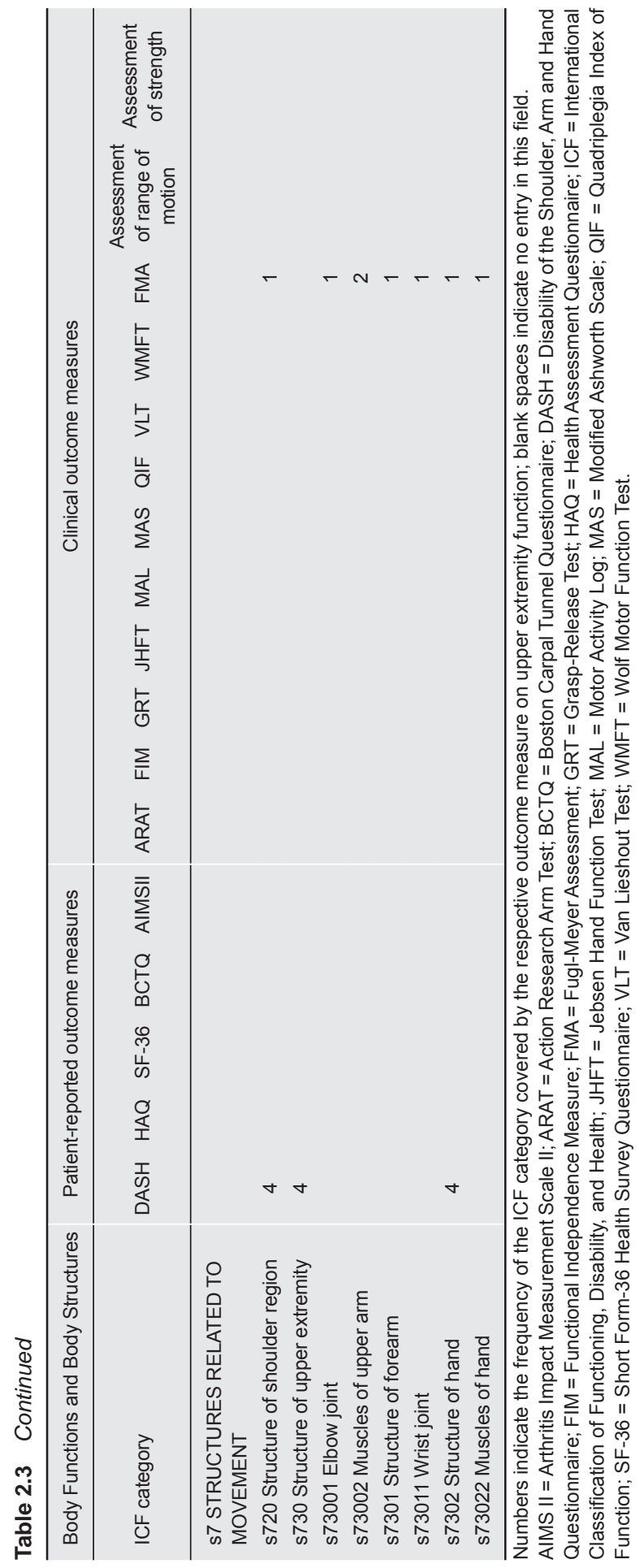



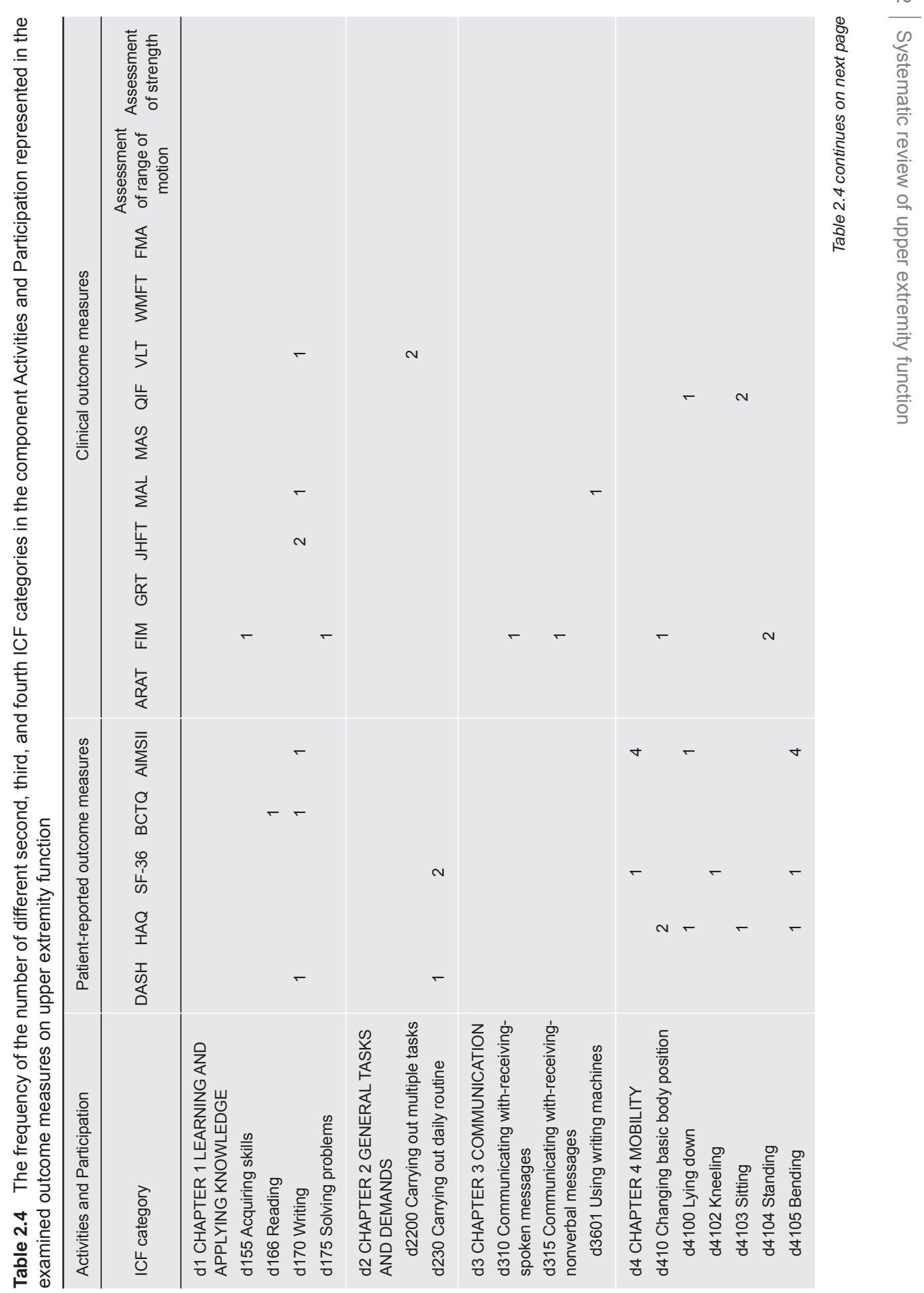


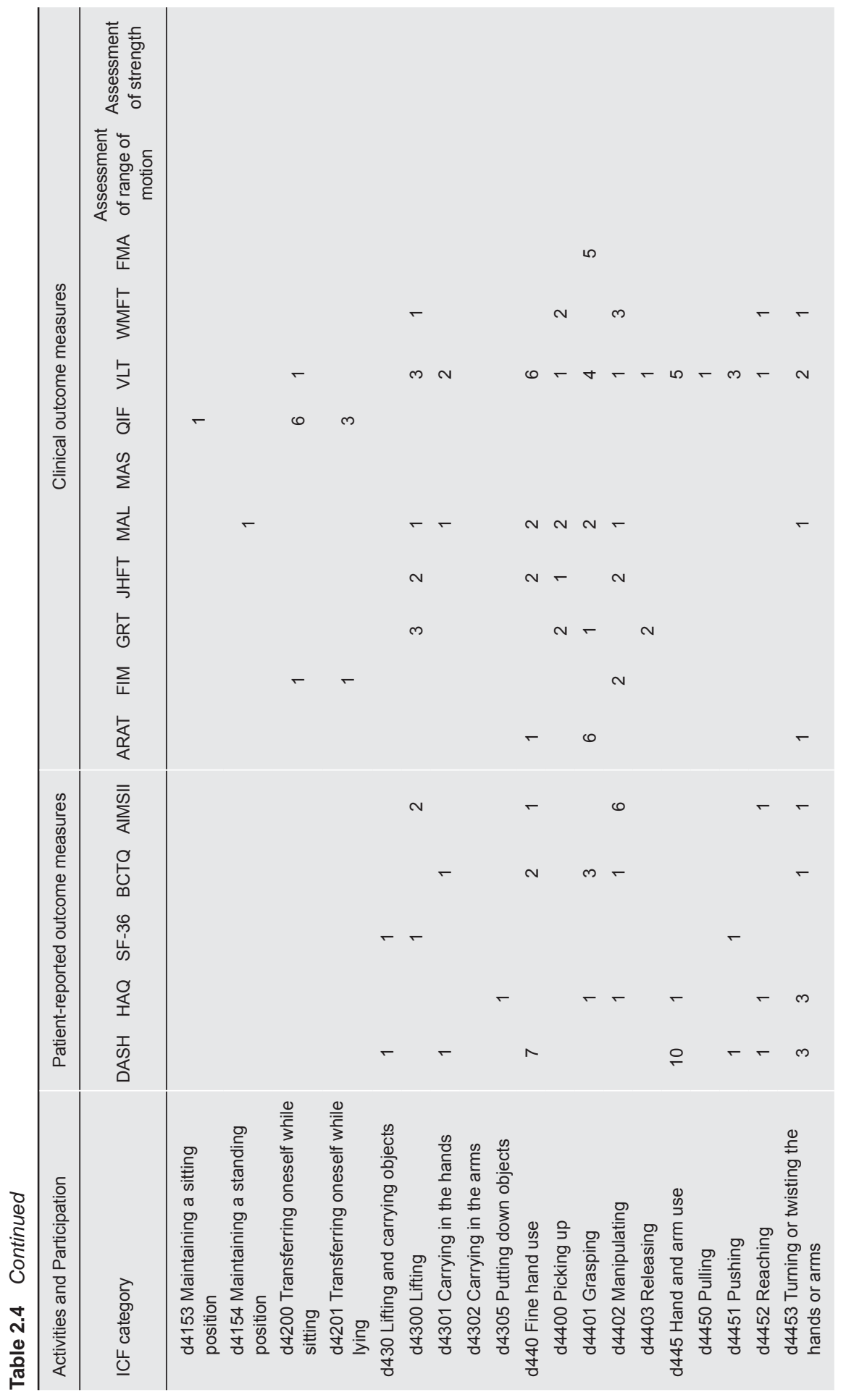




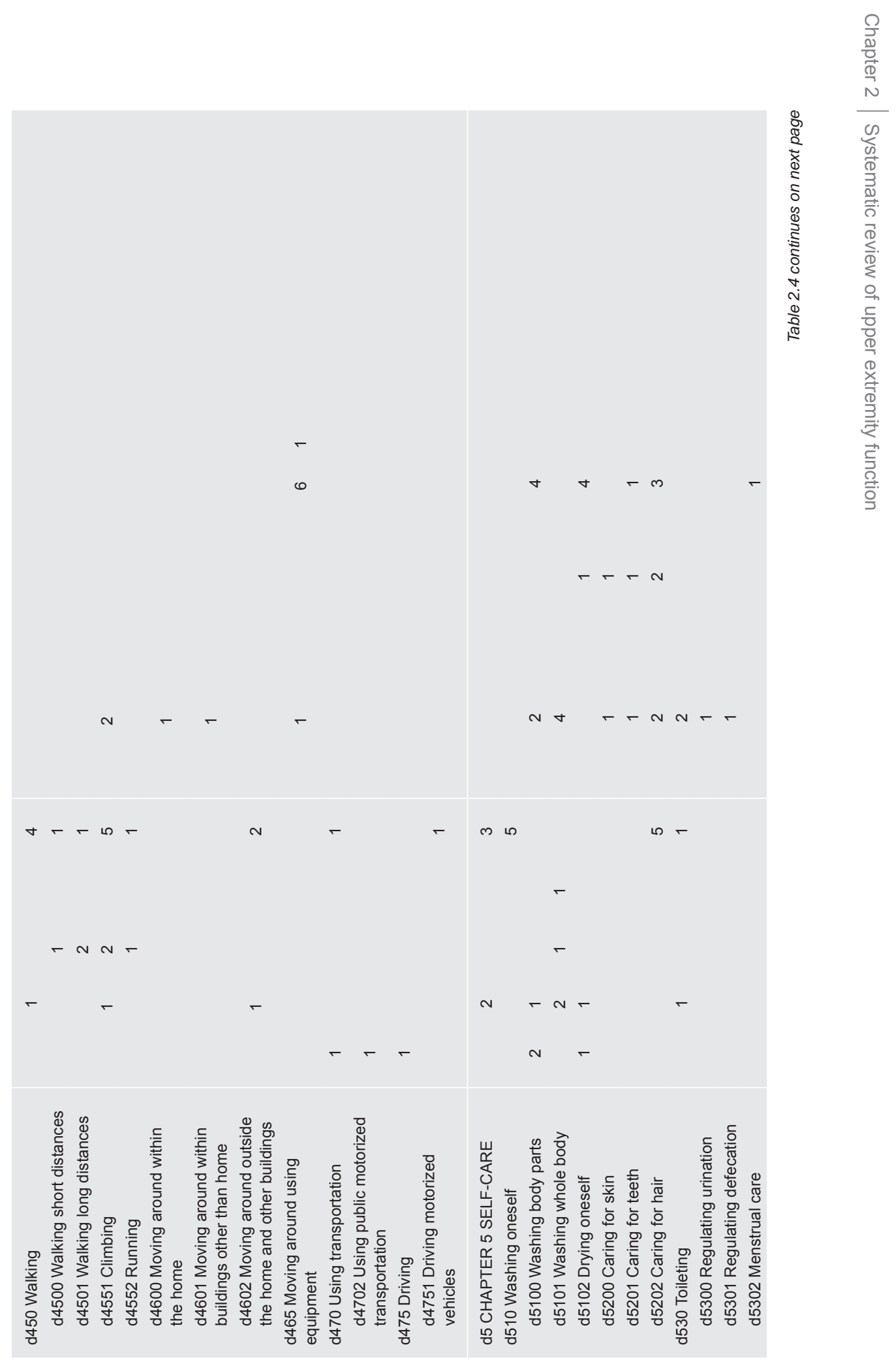




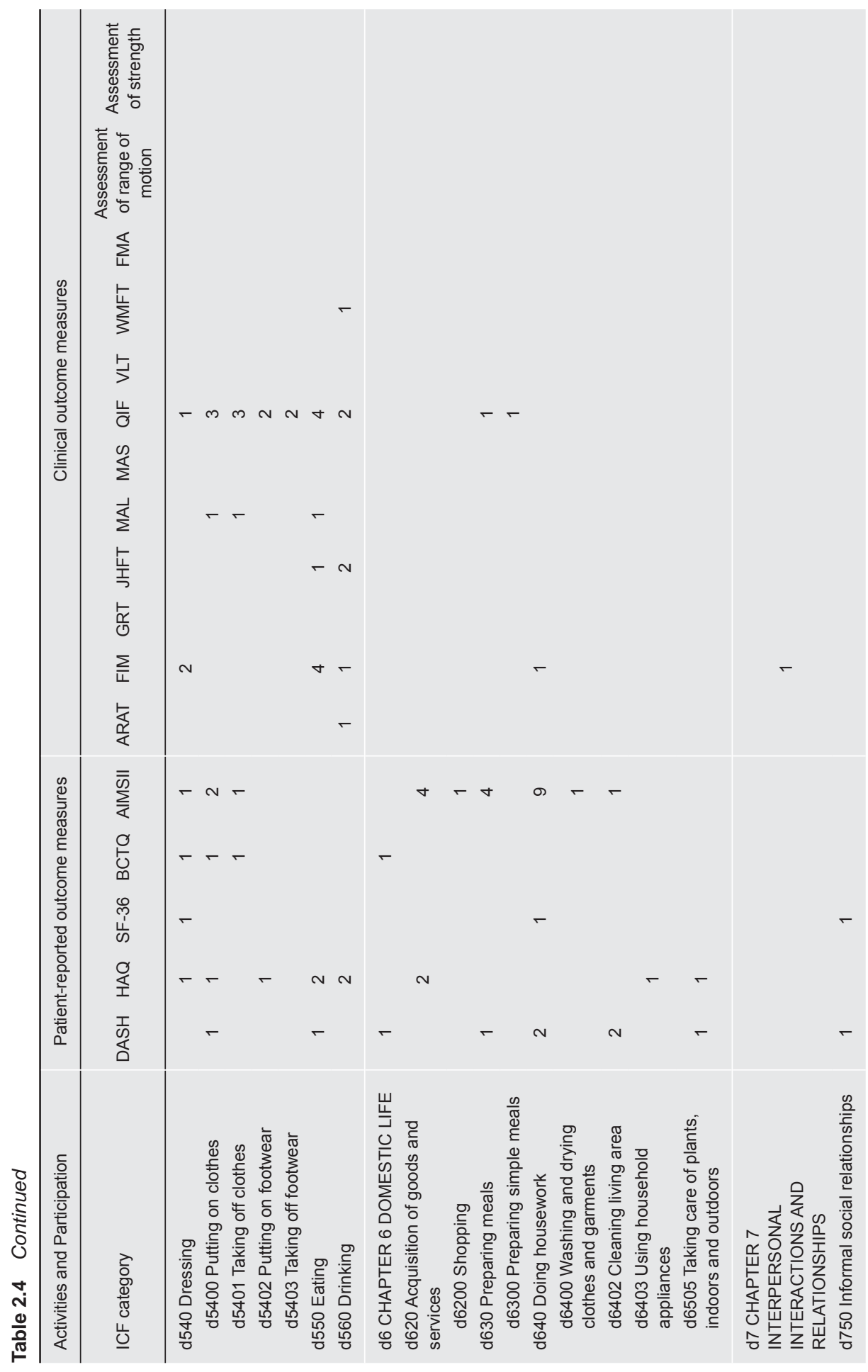




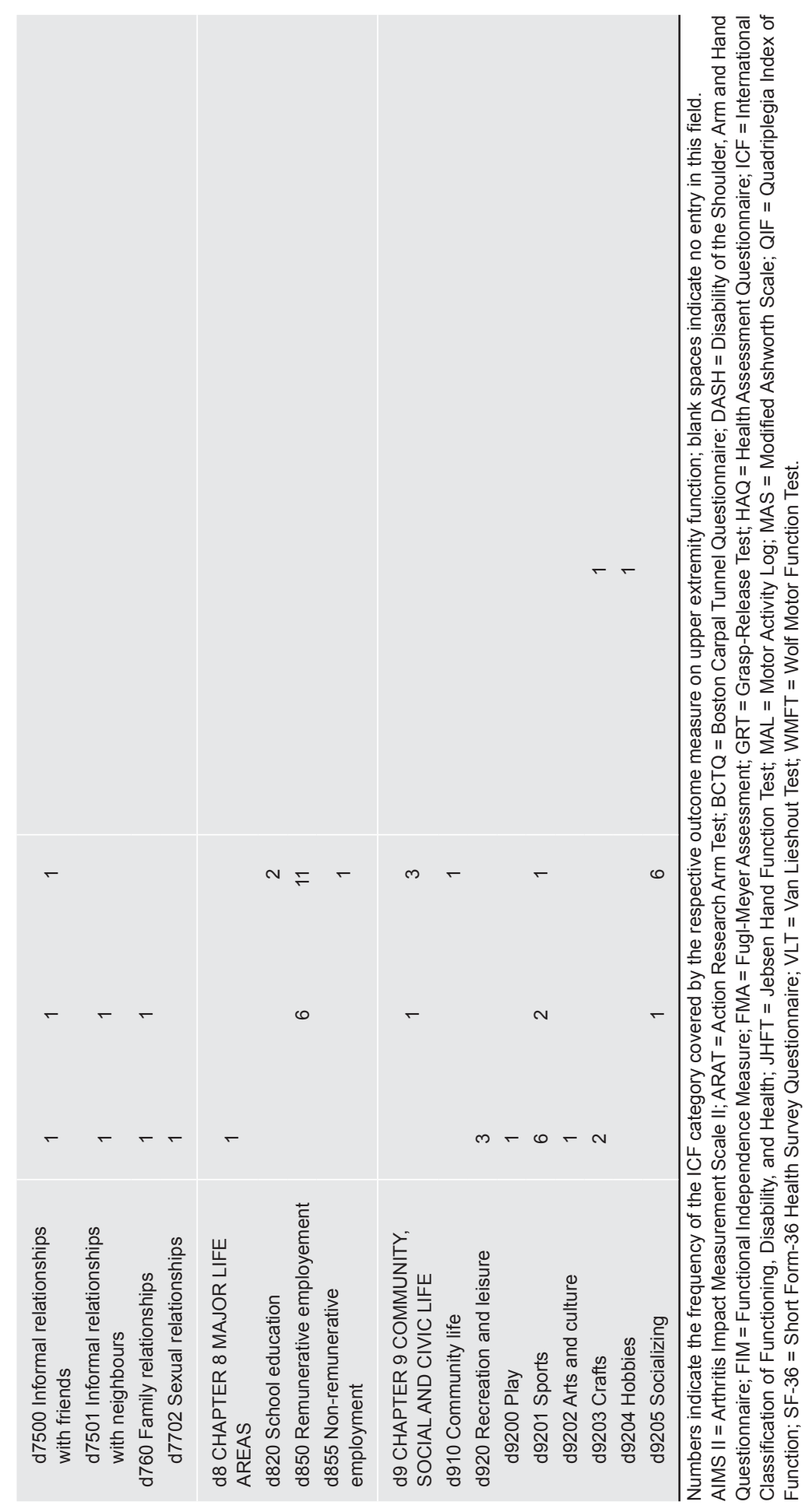




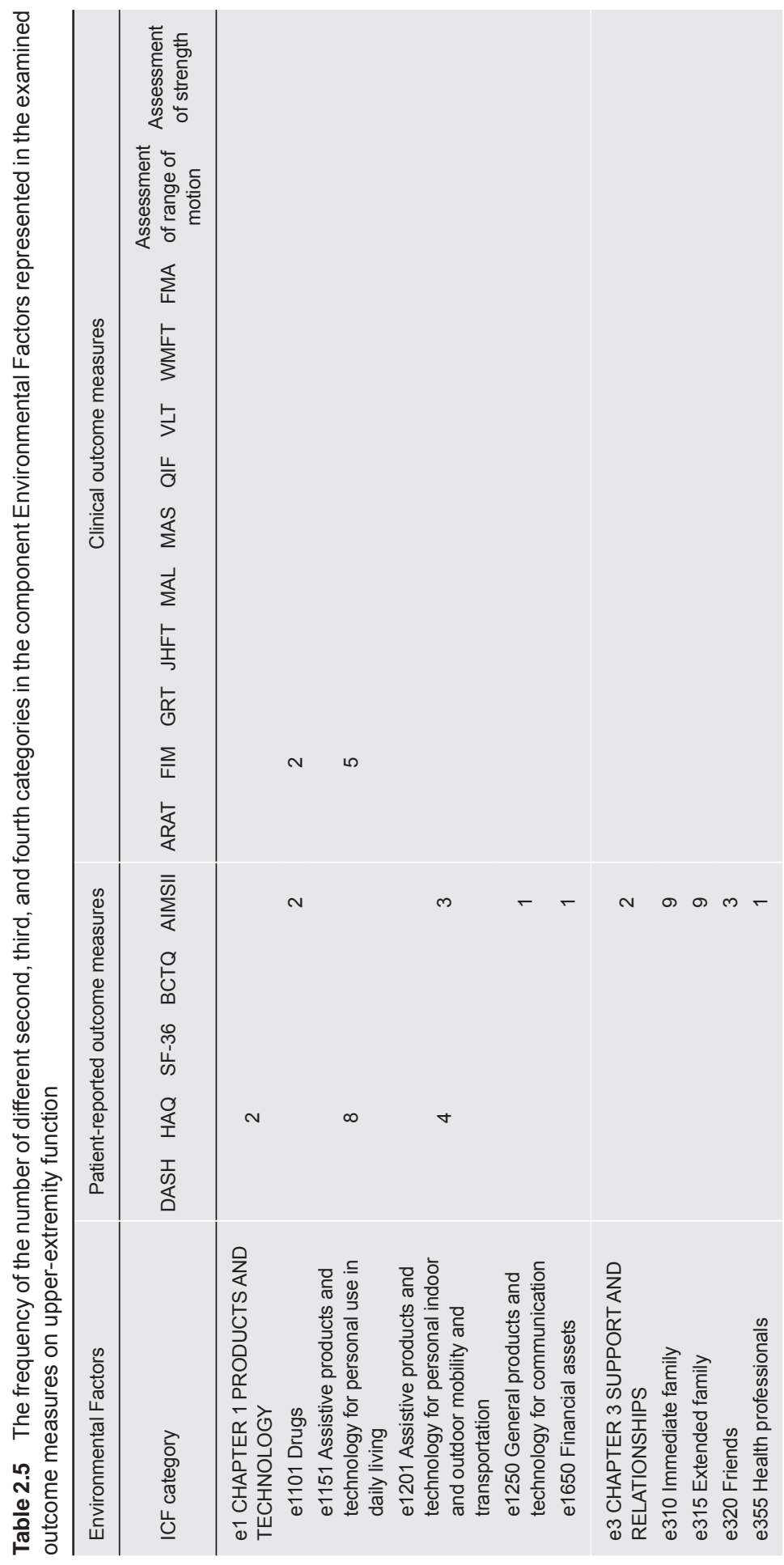



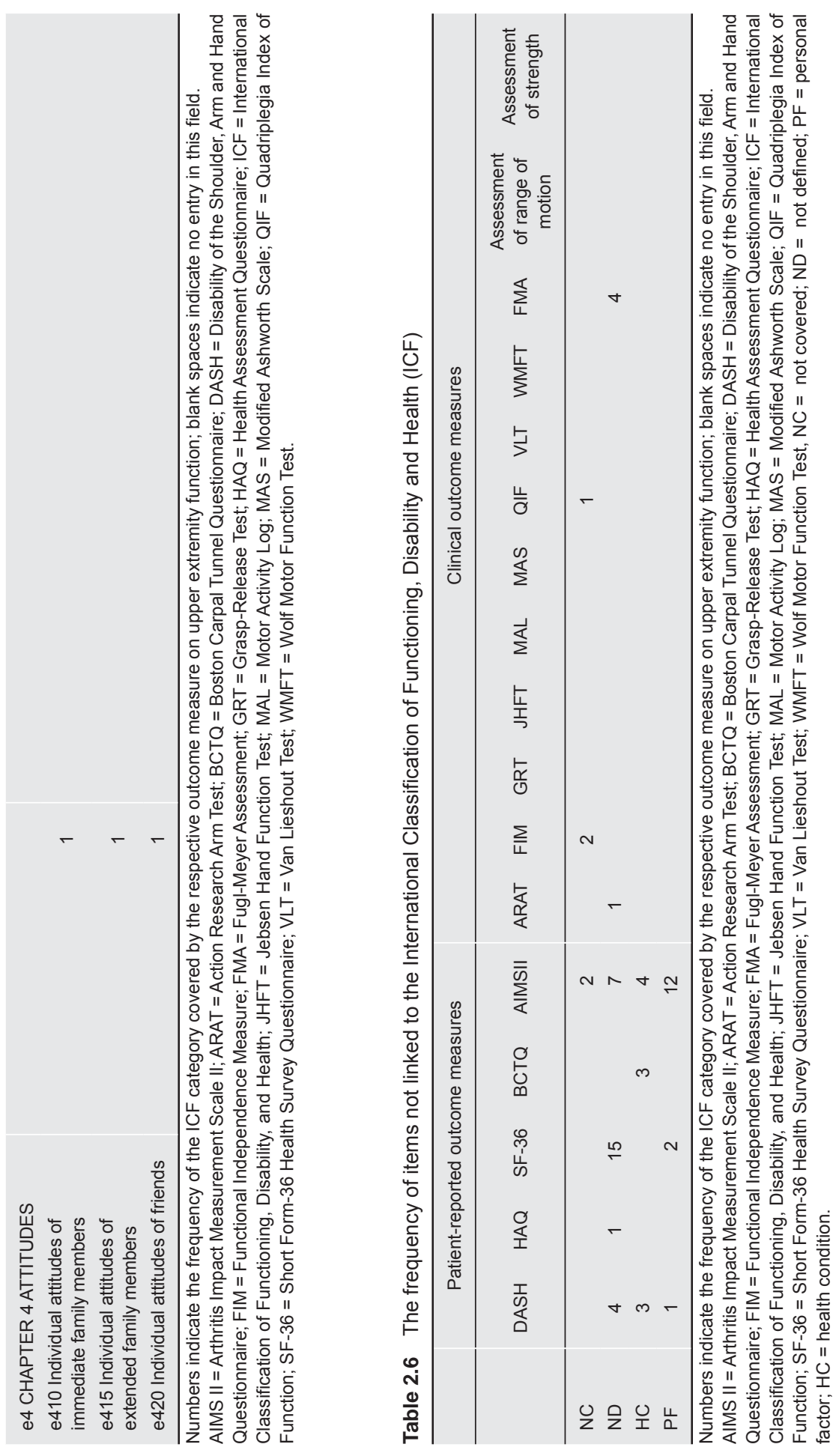
The number of ICF categories from the different chapters represented in the outcome measures ranged from 39 in Chapter 4, Mobility, to 2 in Chapter d2, General Tasks and Demands.

Fourteen ICF categories of the component Environmental Factors are covered in 3 of the 17 outcome measures. Six belong to Chapter e1, Products and Technology, 5 belong to Chapter e3, Support and Relationships; and 3 belong to Chapter e4, Attitudes.

Three outcome measures had content that was considered not covered in the ICF. These measures were the AIMS II, Functional Independence Measure (FIM), ${ }^{36,37}$ and QIF. Six outcome measures had content considered not sufficiently specified to be assigned to a specific ICF category. These measures were Disability of the Shoulder, Arm and Hand Questionnaire (DASH), ${ }^{38} \mathrm{HAQ}$, SF-36, AIMS II, Action Research Arm Test (ARAT), ${ }^{38}$ and the Fugl-Meyer Assessment (FMA). ${ }^{40}$ Three outcome measures addressed personal factors. These measures were the DASH, SF-36, and AIMS II. Two outcome measures cover 2 health conditions, and they were the Severity of Symptoms and Functional Status of the Boston Carpal Tunnel Questionnaire (BCTQ) $)^{41}$ and AIMS II. This information is shown in Table 2.6.

The K statistic (with bootstrapped confidence intervals) was $0.52(0.48-0.55)$ at the first level, $0.47(0.44-0.50)$ at the second level, and $0.45(0.41-0.48)$ at the third level of the classification. No confidence interval includes the value zero, which indicates that the level of agreement is beyond chance.

\section{Discussion}

This literature review provides an overview of outcome measures used to address functioning and disability in persons with impairment in upper extremity function. Moreover, it presents an overview of the content addressed in these outcome measures when the ICF is used as a reference. It was possible to identify outcome measures from a comprehensive perspective rather than from just one specific patient population. We also present information on the reliability and responsiveness of the outcome measures and the populations in which these psychometric properties were studied. Thus this investigation provides clinicians and researchers with a guide for selecting the most appropriate outcome measure for their clinical population or research question, taking ICF-based content validity ("what do the outcome measures address?"), reliability, and responsiveness into consideration.

Patient-reported outcome measures for the upper extremities are most frequently used in rheumatologic diseases and peripheral upper extremity-specific conditions, 
whereas clinical outcome measures are most frequently used in persons who have had a stroke. Outcome assessment in rheumatologic diseases with use of patient-reported outcome measures has a long tradition. In 1993, the Outcome Measures in Rheumatology ${ }^{88}$ emphasized the importance of including patient-recorded outcome measures in rheumatoid arthritis clinical trials. In peripheral upper extremity-specific conditions, the patient's view also is considered an important outcome measure.$^{89-92}$ In persons who have had a stroke, clinicians are still reluctant to use patient-reported outcome measures, which usually reflect the patient's own perception, because of cognitive impairments frequently associated with this health condition. ${ }^{93}$

This review shows that only a few upper extremity-specific measures are used in studies of persons with tetraplegia, and none of them is a patient-reported outcome measure. Information on psychometric properties of outcome measures is lacking for this specific population. ${ }^{16}$ This defiency can be a hindrance for rehabilitation and research in the tetraplegic population because relevant outcome measures are not validated and consequently are not used. For example, the DASH has not yet been validated for tetraplegia, even though it could provide great insight into activity limitations and participation restrictions of persons with tetraplegia. Further research should be performed to study the psychometric properties of the DASH when it is used in the tetraplegic population.

Regarding the content comparison of the specific outcome measures analyzed in this review, different issues require special annotation. In the component Body Functions, all patient-reported outcome measures address mental functions (e.g., maintenance sleep), sensory functions, and pain. Mental functions in upper extremity impairment are major concerns. ${ }^{1-4}$ However, only the FIM as a clinical outcome measure addresses both mental functions and pain. In addition, the FIM also includes body functions that are not related to impairment of upper extremity function such as chewing, swallowing, and fecal and urinary continence..$^{94}$ The Van Lieshout Test ${ }^{42,43}$ exclusively addresses sensory functions. Our work shows that almost all the patient-reported and clinical outcome measures address body functions related to the musculoskeletal system. This finding is consistent with the literature. ${ }^{35,95-97}$

In the Activities and Participation component, all outcome measures comprehensively address Chapter d4, Mobility, except for the Modified Ashworth Scale. ${ }^{44,45}$ However, the $\mathrm{HAQ}$ and the AIMS II address not just aspects of mobility of the upper extremities alone but also of the lower extremities. This finding reflects the fact that both outcome measures were originally developed for patients with rheumatoid arthritis whose lower extremities 
also were affected. ${ }^{9}$ Several outcome measures address ICF category d170, Writing, and the BCTQ also addresses category d166, Reading. It must be considered that the content of outcomes addressing writing was not linked only to the mentioned ICF category d170 but also to the category d440, Fine Hand Use, and d445, Arm Hand Use, because the definition of "writing" provided by the ICF refers exclusively to the cognitive components of this Activity and Participation domain. The same scenario applies to items such as "Holding a Book While Reading" (BCTQ), which was linked to both d166, Reading, and d445, Hand and Arm Use.

Chapter d5, Self-care, is covered by all patient-reported outcome measures, whereas the HAQ and the AIMS II address self-care in a comprehensive way. These 2 measures not only address washing and dressing, as do the other patient-reported measures, but also toileting. In the case of clinical outcome measures, only 3 address self-care, namely the FIM, Motor Activity Log, ${ }^{46}$ and QIF. All of them also cover this chapter in a comprehensive way because they include washing, dressing, toileting, eating, and drinking.

Only 3 patient-reported outcome measures in the component Activity and Participation cover Chapter 8, Major Life Areas, and Chapter 9, Community, Social, and Civic Life, with the exception of the MAL, which covers d9203, Crafts, and d9204, Hobbies. This situation indicates that few outcome measures related to the upper extremity can be used to cover these relevant areas of life, which are usually restricted in the studied populations considered in this review.

In the component Environmental Factors, only 3 outcome measures, the $H A Q$, AIMSII, and FIM, address to some extent the influence of the environment. However, although the HAQ and FIM exclusively address medication and assistive devices, the AIMS II also addresses the social environment and its attitudes, which can have a very relevant positive or negative influence on the lives of persons with upper extremity problems. ${ }^{5,15,100-105}$ Attitudes and support of health professionals, family members, and friends or colleagues are essential in a person's ability to cope with the consequences of the disability. ${ }^{102-105}$ Our results show that current outcome measures lack information on Environmental Factors. Therefore it would be worthwhile to either increase the coverage of Environmental Factors when developing upper extremity function outcome measures or add domains to existing measures.

An important finding of this study is that most information related to patient-reported and clinical outcome measures could be linked to the ICF. The linking option "not covered" was seldom used, which indicates that the ICF is a potentially useful tool for describing 
the problems of functioning and disability associated with the upper extremities with the outcome measures we found. In contrast, the option "not definable" was more frequently used. In particular, this option was used 17 times in the SF-36, because the information "general health" and "physical health" contained in its items were linked to that option. The same situation applies to the items of the AIMS II. It also is apparent that the AIMS II is the outcome measure that contains the most personal factors such as race, age, and gender.

The information regarding reliability and responsiveness shows that for a number of outcome measures, no data are available on responsiveness for different populations, and reliability data also are lacking for different outcome measures and populations (Table 2.2). Thus this overview table can be used to facilitate the selection process of outcome measures for investigations or clinical practice and provides an indication of the areas of upper extremity outcome measures in which future research is needed.

\section{Limitations}

The current review is subject to some limitations. We excluded case series; the minimum sample size was 10; and the minimum age of subjects was 18 years. These exclusions must be considered when interpreting the results of this review. An additional limitation is that we considered only outcome measures for which a reference or information on the psychometric properties were provided. This approach could have biased the frequency with which the outcome measures were identified. However, it may encourage authors of scientific publications to always properly reference the outcome measures used in their studies. Furthermore, we included the 5 most frequently used outcome measures in each of the study populations. Outcome measures developed in recent years that have not yet been frequently used, such as the Graded and Redefined Assessment of Strength, Sensibility and Prehension, ${ }^{106}$ were not considered in this review. On the basis of the $\mathrm{K}$ coefficient and the lower limits of the confidence intervals, the degree of agreement between health professionals is acceptable.

\section{Conclusion}

In conclusion, the ICF provides a useful reference for identifying the content validity of outcome measures used to address functioning and disability in persons with impairment in upper extremity function. This overview can be helpful when planning studies, deciding which outcome measure to use in clinical practice, and determining whether development of a new outcome measure is necessary. In clinical practice, the selection of outcome 
measures can properly capture change resulting from an intervention, and in research, a proper outcome measure can provide answers to a research question. For example, before deciding to develop a new outcome measure for tetraplegia, the content of outcome measures used for other specific conditions should be consulted. However, not only the content is relevant when selecting outcome measures or deciding whether the development of a new outcome measure is necessary; psychometric properties such as reliability and responsiveness also are relevant. Therefore reliability and responsiveness data of the outcome measures selected were also provided in this study.

Our results show that reliability and responsiveness data are not always available for the populations in which the outcome measures have been used. In selecting appropriate outcome measures, one needs to consider clinical and patient-reported measures with broad coverage of the domains of functioning. Such a selection will depend on the research question and study design.

The findings from our study show that research with a wider focus is needed to encompass the multifaceted problems experienced by persons with upper extremity function impairment. It is therefore important that outcome measures related to upper extremity function capture the entire spectrum of functioning and disability.

\section{Acknowlegements}

We are grateful to Reuben Escorpizo, Alexandra Rauch, and Stefan van Drongelen from Swiss Paraplegic Research, Nottwil, Switzerland, and to Edda Amann, Christina Bostan, Heinrich Gall, Barbara Kollerits, and Sandra Kus from the Research Unit of Biopsychosocial Health at the Institute for Health and Rehabilitation Sciences at the Ludwig-MaximiliansUniversity, Munich, Germany, for their valuable contribution and support in all different steps of this study.

\section{References}

1. Stamm T, van der Giesen F, Thorstensson C, et al. Patient perspective of hand osteoarthritis in relation to concepts covered by instruments measuring functioning: A qualitative European multicentre study. Ann Rheum Dis 2009;68:1453-1460.

2. Jerosch-Herold C, Mason R, Chojnowski AJ. A qualitative study of the experiences and expectations of surgery in patients with carpal tunnel syndrome. J Hand Ther 2008;21:54-61.

3. Gustafsson M, Ahlstrom G. Emotional distress and coping in the early stage of recovery following acute traumatic hand injury: A questionnaire survey. Int J Nurs Stud 2006;43:557-565. 
4. Carlsson IK, Edberg AK, Wann-Hansson C. Hand-injured patients' experiences of cold sensitivity and the consequences and adaptation for daily life: A qualitative study. J Hand Ther 2010;23:5361.

5. Grunert BK, Devine CA, Matloub HS, et al. Psychological adjustment following work-related hand injury: 18-month follow-up. Ann Plast Surg 1992;29:537-542.

6. Gustafsson M, Ahlstrom G. Problems experienced during the first year of an acute traumatic hand injury—a prospective study. J Clin Nurs 2004;13:986-995.

7. Larsson TJ, Bjornstig U. Persistent medical problems and permanent impairment five years after occupational injury. Scand J Soc Med 1995;23:121-128.

8. Nakayama H, Jorgensen HS, Raaschou HO, Olsen TS. Recovery of upper extremity function in stroke patients: The Copenhagen Stroke Study. Arch Phys Med Rehabil 1994;75:394-398.

9. Ewert T, FuessI M, Cieza A, et al. Identification of the most common patient problems in patients with chronic conditions using the ICF checklist. J Rehabil Med 2004;44S:186-188.

10. Anderson KD. Targeting recovery: Priorities of the spinal cord-injured population. J Neurotrauma 2004;21:1371-1383.

11. Snoek GJ, IJzerman MJ, Hermens HJ, Maxwell D, Biering-Sorensen F. Survey of the needs of patients with spinal cord injury: Impact and priority for improvement in hand function in tetraplegics. Spinal Cord 2004;42:526-532.

12. Trybus M, Lorkowski J, Brongel L, Hladki W. Causes and consequences of hand injuries. Am J Surg 2006;192:52-57.

13. Rosberg HE, Carlsson KS, Dahlin LB. Prospective study of patients with injuries to the hand and forearm: Costs, function, and general health. Scand J Plast Reconstr Surg Hand Surg 2005;39:360-369.

14. Malcus Johnsson P, Sandqvist G, Bengtsson A, Nived O. Hand function and performance of daily activities in systemic lupus erythematosus. Arthritis Rheum 2008;59:1432-1438.

15. Kjeken I, Dagfinrud H, Slatkowsky-Christensen B, et al. Activity limitations and participation restrictions in women with hand osteoarthritis: Patients' descriptions and associations between dimensions of functioning. Ann Rheum Dis 2005;64:1633-1638.

16. van Tuijl JH, Janssen-Potten YJ, Seelen HA. Evaluation of upper extremity motor function tests in tetraplegics. Spinal Cord 2002;40: 51-64.

17. van de Ven-Stevens LA, Munneke M, Terwee CB, Spauwen PH, van der Linde H. Clinimetric properties of instruments to assess activities in patients with hand injury: A systematic review of the literature. Arch Phys Med Rehabil 2009;90:151-169.

18. Schoneveld K, Wittink H, Takken T. Clinimetric evaluation of measurement tools used in hand therapy to assess activity and participation. J Hand Ther 2009;22:221-235.

19. Changulani M, Okonkwo U, Keswani T, Kalairajah Y. Outcome evaluation measures for wrist and hand: Which one to choose? Int Orthop 2008;32:1-6.

20. Bindra RR, Dias JJ, Heras-Palau C, Amadio PC, Chung KC, Burke FD. Assessing outcome after hand surgery: The current state. J Hand Surg Br 2003;28:289-294.

21. Okkema KA, Culler KH. Functional evaluation of upper extremity use following stroke: A literature review. Top Stroke Rehabil 1998;4:54-75.

22. World Health Organization. International Classification of Functioning, Disability and Health. 2001. Available at http://www.who.int/classifications/icf/en/. Accessed March 31, 2011. 
23. Cieza A, Brockow T, Ewert $\mathrm{T}$, et al. Linking health-status measurements to the international classification of functioning, disability and health. J Rehabil Med 2002;34:205-210.

24. Cieza A, Geyh S, Chatterji S, Kostanjsek N, Ustun B, Stucki G. ICF linking rules: An update based on lessons learned. J Rehabil Med 2005;37:212-218.

25. Boers M, Brooks $P$, Strand CV, Tugwell P. The OMERACT filter for outcome measures in rheumatology. J Rheumatol 1998;25:198-199.

26. Fries JF, Spitz P, Kraines RG, Holman HR. Measurement of patient outcome in arthritis. Arthritis Rheum 1980;23:137-145.

27. Marino RJ, Goin JE. Development of a short-form Quadriplegia Index of Function scale. Spinal Cord 1999;37:289-296.

28. Gresham GE, Labi ML, Dittmar SS, Hicks JT, Joyce SZ, Stehlik MA. The Quadriplegia Index of Function (QIF): Sensitivity and reliability demonstrated in a study of thirty quadriplegic patients. Paraplegia 1986;24:38-44.

29. Wuolle KS, Van Doren CL, Thrope GB, Keith MW, Peckham PH. Development of a quantitative hand grasp and release test for patients with tetraplegia using a hand neuroprosthesis. J Hand Surg Am 1994;19:209-218.

30. Karlsson G. Psychological qualitative Research From a Phenomenological Perspective. Stockholm, Sweden: Almquist \& Wiskell International,1995.

31. Bullinger M. [Assessment of health related quality of life with the SF-36 Health Survey]. Rehabilitation (Stuttg) 1996;35:XVII-XXVII.

32. Ware JE Jr, Sherbourne CD. The MOS 36-item short-form health survey (SF-36). I. Conceptual framework and item selection. Med Care 1992;30:473-483.

33. Meenan RF, Gertman PM, Mason JH. Measuring health status in arthritis. The arthritis impact measurement scales. Arthritis Rheum 1980;23:146-152.

34. Meenan RF, Mason JH, Anderson JJ, Guccione AA, Kazis LE. AIMS2. The content and properties of a revised and expanded Arthritis Impact Measurement Scales Health Status Questionnaire. Arthritis Rheum 1992;35:1-10.

35. Casanova JS, ed. Clinical Assessement Recommendations. 2nd ed. Chicago, IL: American Society of Hand Therapists, 1992.

36. Hamilton BB, Laughlin JA, Fiedler RC, Granger CV. Interrater reliability of the 7-level functional independence measure (FIM). Scand J Rehabil Med 1994;26:115-119.

37. Granger CV, Hamilton BB, Keith RA, Zielezny M, Sherwin FS. Advances in functional assessment for medical rehabilitation. Top Geriatr Rehabil 1986;1:59-74.

38. Hudak PL, Amadio PC, Bombardier C. Development of an upper extremity outcome measure: The DASH (disabilities of the arm, shoulder and hand) [corrected]. The Upper Extremity Collaborative Group (UECG). Am J Ind Med 1996;29:602-608.

39. Lyle RC. A performance test for assessment of upper limb function in physical rehabilitation treatment and research. Int J Rehabil Res 1981;4:483-492.

40. Fugl-Meyer AR, Jääskö L, Leyman I, Olsson S, Steglind S. The poststroke hemiplegic patient. 1. A method for evaluation of physical performance. Scand J Rehabil Med 1975;7:13-31.

41. Levine DW, Simmons BP, Koris MJ, et al. A self-administered questionnaire for the assessment of severity of symptoms and functional status in carpal tunnel syndrome. J Bone Joint Surg Am 1993;75:1585-1592. 
42. Post MW, Van Lieshout G, Seelen HA, Snoek GJ, ljzerman MJ, Pons C. Measurement properties of the short version of the Van Lieshout test for arm/hand function of persons with tetraplegia after spinal cord injury. Spinal Cord 2006;44:763-771.

43. Spooren Al, Janssen-Potten YJ, Post MW, Kerckhofs E, Nene A, Seelen HA. Measuring change in arm hand skilled performance in persons with a cervical spinal cord injury: Responsiveness of the Van Lieshout Test. Spinal Cord 2006;44:772-779.

44. Ashworth B. Preliminary trial of carisoprodol in multiple sclerosis. Practitioner 1964;192:540542.

45. Bohannon RW, Smith MB. Interrater reliability of a modified Ashworth scale of muscle spasticity. Phys Ther 1987;67:206-207.

46. Carr JH, Shepherd RB, Nordholm L, Lynne D. Investigation of a new motor assessment scale for stroke patients. Phys Ther 1985;65:175-180.

47. Wolf SL, Lecraw DE, Barton LA, Jann BB. Forced use of hemiplegic upper extremities to reverse the effect of learned nonuse among chronic stroke and head-injured patients. Exp Neurol 1989;104:125-132.

48. Jebsen RH, Taylor N, Trieschmann RB, Trotter MJ, Howard LA. An objective and standardized test of hand function. Arch Phys Med Rehabil 1969;50:311-319.

49. Stern EB. Stability of the Jebsen-Taylor Hand Function Test across three test sessions. Am J Occup Ther 1992;46:647-649.

50. Cohen J. A coefficient of agreement for nominal scales. Educ Psychol Meas 1960;20:37-46.

51. Vierkant RA. A SAS $\otimes$ macro for calculating bootstrapped confidence intervals about a Kappa coefficient. SAS Users Group International Online Proceedings, 2000. Available at http://www2. sas.com/proceedings/sugi22/STATS/PAPER295.PDF. Accessed March 31, 2011.

52. Blackburn M, van Vliet $P$, Mockett SP. Reliability of measurements obtained with the modified Ashworth scale in the lower extremities of people with stroke. Phys Ther 2002;82:25-34.

53. Brashear A, Zafonte R, Corcoran M, et al. Inter- and intrarater reliability of the Ashworth Scale and the Disability Assessment Scale in patients with upper-limb poststroke spasticity. Arch Phys Med Rehabil 2002;83:1349-1354.

54. Gregson JM, Leathley MJ, Moore AP, Smith TL, Sharma AK, Watkins CL. Reliability of measurements of muscle tone and muscle power in stroke patients. Age Ageing 2000;29:223228.

55. Bodin P, Morris ME. Inter-rater reliability of the modified Ashworth scale for spasticity in hemiplegic patients. In: Proceedings of the 11th Congress of the World Federation of Physical Therapy. London, England: World Federation of Physical Therapy, 1991, 505-507.

56. Nijland R, van Wegen E, Verbunt J, van Wijk R, van Kordelaar J, Kwakkel G. A comparison of two validated tests for upper limb function after stroke: The Wolf Motor Function Test and the Action Research Arm Test. J Rehabil Med 2010;42:694-696.

57. Beebe JA, Lang CE. Relationships and responsiveness of six upper extremity function tests during the first six months of recovery after stroke. J Neurol Phys Ther 2009;33:96-103.

58. Hammer AM, Lindmark B. Responsiveness and validity of the Motor Activity Log in patients during the subacute phase after stroke. Disabil Rehabil 2010;32:1184-1193.

59. Uswatte G, Taub E, Morris D, Vignolo M, McCulloch K. Reliability and validity of the upper extremity Motor Activity Log-14 for measuring real-world arm use. Stroke 2005;36:2493-2496. 
60. Morris DM, Uswatte G, Crago JE, Cook EW III, Taub E. The reliability of the wolf motor function test for assessing upper extremity function after stroke. Arch Phys Med Rehabil 2001;82:750755.

61. Schepers VP, Ketelaar M, Visser-Meily JM, Dekker J, Lindeman E. Responsiveness of functional health status measures frequently used in stroke research. Disabil Rehabil 2006;28:1035-1040.

62. Fricke J, Unsworth C, Worrell D. Reliability of the Functional Independence Measure with occupational therapists. Austr Occup Ther J 1993;40:7-15.

63. Segal ME, Ditunno JF, Staas WE. Interinstitutional agreement of individual functional independence measure (FIM) items measured at two sites on one sample of SCl patients. Paraplegia 1993;31:622-631.

64. Vliet Vlieland TP, van der Wijk TP, Jolie IM, Zwinderman AH, Hazes JM. Determinants of hand function in patients with rheumatoid arthritis. J Rheumatol 1996;23:835-840.

65. Mulcahey MJ, Smith BT, Betz RR. Psychometric rigor of the Grasp and Release Test for measuring functional limitation of persons with tetraplegia: A preliminary analysis. J Spinal Cord Med 2004;27:41-46.

66. Labi MLC, Dittmar SS, Hicks JT, Joyce SZ, Phillips MA, Gresham GE. Quadriplegia index of function one year follow-up. Arch Phys Med Rehabil 1981;62:532-533.

67. Woodbury ML, Velozo CA, Richards LG, Duncan PW, Studenski S, Lai SM. Longitudinal stability of the Fugl-Meyer Assessment of the upper extremity. Arch Phys Med Rehabil 2008;89:15631569.

68. Sanford J, Moreland J, Swanson LR, Stratford PW, Gowland C. Reliability of the Fugl-Meyer assessment for testing motor performance in patients following stroke. Phys Ther 1993;73:447454.

69. Duncan PW, Propst M, Nelson SG. Reliability of the Fugl-Meyer assessment of sensorimotor recovery following cerebrovascular accident. Phys Ther 1983;63:1606-1610.

70. Almborg AH, Berg S. Quality of life among Swedish patients after stroke: Psychometric evaluation of SF-36. J Rehabil Med 2009;41:48-53.

71. Hagen S, Bugge C, Alexander H. Psychometric properties of the SF-36 in the early post-stroke phase. J Adv Nurs 2003;44:461-468.

72. Dorman P, Slattery J, Farrell B, Dennis M, Sandercock P. Qualitative comparison of the reliability of health status assessments with the EuroQol and SF-36 questionnaires after stroke. United Kingdom Collaborators in the International Stroke Trial. Stroke 1998;29:63-68.

73. Anderson C, Laubscher S, Burns R. Validation of the Short Form 36 (SF-36) health survey questionnaire among stroke patients. Stroke1996;27:1812-1816.

74. Leung YY, Ho KW, Zhu TY, Tam LS, Kun EW, Li EK. Testing scaling assumptions, reliability and validity of medical outcomes study shortform 36 health survey in psoriatic arthritis. Rheumatology (Oxford) 2010;49:1495-1501.

75. Kvien TK, Kaasa S, Smedstad LM. Performance of the Norwegian SF-36 Health Survey in patients with rheumatoid arthritis. II. A comparison of the SF-36 with disease-specific measures. J Clin Epidemiol 1998;51:1077-1086.

76. Husted JA, Gladman DD, Farewell VT, Long JA, Cook RJ. Validating the SF-36 health survey questionnaire in patients with psoriatic arthritis. J Rheumatol 1997;24:511-517.

77. Beaton D, Richards RR. Assessing the reliability and responsiveness of 5 shoulder questionnaires. J Shoulder Elbow Surg 1998;7:565-572. 
78. Lautenschläger J, Mau W, Kohlmann W, et al. Vergleichende Evaluation einer deutschen Version des Health Assessment Questionnaires (HAQ) und des Functionsfragebogens Hannover (FFbH). Zeitschrift für Rheumatologie 1997;56:144-155.

79. Hobby JL, Watts C, Elliot D. Validity and responsiveness of the patient evaluation measure as an outcome measure for carpal tunnel syndrome. J Hand Surg Br 2005;30:350-354.

80. Greenslade JR, Mehta RL, Belward P, Warwick DJ. Dash and Boston questionnaire assessment of carpal tunnel syndrome outcome: What is the responsiveness of an outcome questionnaire? J Hand Surg Br 2004;29:159-164.

81. Gummesson C, Atroshi I, Ekdahl C. The disabilities of the arm, shoulder and hand (DASH) outcome questionnaire: Longitudinal construct validity and measuring self-rated health change after surgery. BMC Musculoskelet Disord 2003;4:11.

82. Gay RE, Amadio PC, Johnson JC. Comparative responsiveness of the disabilities of the arm, shoulder, and hand, the carpal tunnel questionnaire, and the SF-36 to clinical change after carpal tunnel release. J Hand Surg Am 2003;28:250-254.

83. Beaton DE, Katz JN, Fossel AH, Wright JG, Tarasuk V, Bombardier C. Measuring the whole or the parts? Validity, reliability, and responsiveness of the Disabilities of the Arm, Shoulder and Hand outcome measure in different regions of the upper extremity. J Hand Ther 2001;14:128146.

84. Veehof MM, Sleegers EJ, van Veldhoven NH, Schuurman AH, van Meeteren NL. Psychometric qualities of the Dutch language version of the Disabilities of the Arm, Shoulder, and Hand questionnaire (DASHDLV). J Hand Ther 2002;15:347-354.

85. Christie A, Hagen KB, Mowinckel P, Dagfinrud H. Methodological properties of six shoulder disability measures in patients with rheumatic diseases referred for shoulder surgery. J Shoulder Elbow Surg 2009;18:89-95.

86. Atroshi I, Johnsson R, Sprinchorn A. Self-administered outcome instrument in carpal tunnel syndrome. Reliability, validity and responsiveness evaluated in 102 patients. Acta Orthop Scand 1998;69:82-88.

87. Goossens PH, Heemskerk B, van Tongeren J, Zwinderman AH, Vliet Vlieland TP, Huizinga TW. Reliability and sensitivity to change of various measures of hand function in relation to treatment of synovitis of the metacarpophalangeal joint in rheumatoid arthritis. Rheumatology (Oxford) 2000;39:909-913.

88. Tugwell $\mathrm{P}$, Boers M. OMERACT conference on outcome measures in rheumatoid arthritis clinical trials: Introduction. J Rheumatol 1993;20:528-530.

89. Amadio PC. Outcomes assessment in hand surgery. What's new? Clin Plast Surg 1997;24:191194.

90. Bullinger M. [Health related quality of life and subjective health. Overview of the status of research for new evaluation criteria in medicine]. Psychother Psychosom Med Psychol 1997; 47:76-91.

91. Jester A, Harth A, Wind G, Germann G, Sauerbier M. Disabilities of the arm, shoulder and hand (DASH) questionnaire: Determining functional activity profiles in patients with upper extremity disorders. J Hand Surg Br 2005;30:23-28.

92. McAuliffe JA. Outcome research: definitions and directions. J Hand Ther 1998;11:164-170. 
93. Geyh S, Cieza A, Kollerits B, Grimby G, Stucki G. Content comparison of health-related quality of life measures used in stroke based on the international classification of functioning, disability and health (ICF): A systematic review. Qual Life Res 2007;16:833-851.

94. Grill E, Stucki G, Scheuringer M, Melvin J. Validation of International Classification of Functioning, Disability, and Health (ICF) Core Sets for early postacute rehabilitation facilities: Comparisons with three other functional measures. Am J Phys Med Rehabil 2006;85:640-649.

95. Hughes SL, Gibbs J, Edelman P, Singer R, Chang RW. Joint impairment and hand function in the elderly. J Am Geriatr Soc 1992;40:871-877.

96. Ditunno JF Jr, Cohen ME, Hauck WW, Jackson AB, Sipski ML. Recovery of upper extremity strength in complete and incomplete tetraplegia: A multicenter study. Arch Phys Med Rehabil 2000;81:389-393.

97. Bertrand AM, Mercier C, Bourbonnais D, Desrosiers J, Gravel D. Reliability of maximal static strength measurements of the arms in subjects with hemiparesis. Clin Rehabil 2007;21:248257.

98. de Mos M, Huygen FJ, van der Hoeven-Borgman M, Dieleman JP, Ch Stricker BH, Sturkenboom MC. Outcome of the complex regional pain syndrome. Clin J Pain 2009;25:590-597.

99. Martimo KP, Shiri R, Miranda H, Ketola R, Varonen H, Viikari-Juntura E. Self-reported productivity loss among workers with upper extremity disorders. Scand J Work Environ Health 2009;35:301308.

100. Strating MM, van Schuur WH, Suurmeijer TP. Contribution of partner support in self-management of rheumatoid arthritis patients. An application of the theory of planned behavior. J Behav Med 2006;29:51-60.

101. Strating MM, Suurmeijer TP, van Schuur WH. Disability, social support, and distress in rheumatoid arthritis: Results from a thirteen year prospective study. Arthritis Rheum 2006;55:736-744.

102. Lai $\mathrm{CH}$. Motivation in hand-injured patients with and without work-related injury. J Hand Ther 2004;17:6-17.

103. Katz JN, Amick BC III, Keller R, et al. Determinants of work absence following surgery for carpal tunnel syndrome. Am J Ind Med 2005;47:120-130.

104. Evers AW, Kraaimaat FW, Geenen R, Jacobs JW, Bijlsma JW. Pain coping and social support as predictors of long-term functional disability and pain in early rheumatoid arthritis. Behav Res Ther 2003;41:1295-1310.

105. Afshar A, Afshar N. Long-term follow-up evaluation of bilateral total hand loss. J Hand Surg Am 2007;32:1148-1153.

106 Kalsi-Ryan S, Curt A, Fehlings MM, Verrier MC. Assessment of the hand in tetraplegia using the Graded Redefined Assessment of Strength, Sensibility and Prehension (GRASSP): Impairment versus function. Top Spinal Cord Inj Rehabil 2009;14:34-46. 

Changes in strength, sensation and prehension in acute cervical spinal cord injury: European multicenter responsiveness study of the Graded Redefined Assessment of Strength, Sensibility and Prehension (GRASSP)

Inge-Marie Velstra Armin Curt Angela Frotzler Rainer Abel Sukhvinder Kalsi-Ryan Johan S. Rietman Marc Bolliger 


\section{Abstract}

Objective: To investigate the internal and external responsiveness and recovery profiles of the Graded Redefined Assessment of Strength, Sensibility, and Prehension (GRASSP) instrument in revealing changes in upper limb function within the first year following cervical spinal cord injury (SCI).

Method: A European prospective, longitudinal, multicenter study assessing the GRASSP at 1, 3, 6, and 12 months after cervical SCI. Subtests of GRASSP were compared to the upper extremity motor (UEMS) and light touch scores (LT) according to the International Standards of Neurological Classification of Spinal Cord Injury (ISNCSCI), the Spinal Cord Independence Measure self-care subscore (SCIM-SS), as well as a clinician-rated outcome measure (CROM) of clinical relevance. Data were analyzed for GRASSP responsiveness and recovery rate over time.

Results: Seventy-four participants entered the study. GRASSP subtests proved responsive (standardized response mean [SRM] ranged from 0.79 to 1.48 for strength, 0.50 to 1.03 for prehension, and 0.14 to 0.64 for sensation) between all examination time points. In comparison, UEMS and LT showed lower responsiveness (SRM UEMS ranged from 0.69 to 1.29 and SRM LT ranged from 0.30 to -0.13 ). All GRASSP subtests revealed significant, moderate-to-excellent correlations with UEMS, LT, and SCIM-SS at each time point, and changes in GRASSP subtests were in accordance with the CROM. GRASSP prehension and motor recovery was largest between 1 and 3 months. Conclusion: The GRASSP showed excellent responsiveness, detecting distinct changes in strength and prehension relating to the severity of cervical SCI. It detected clinically significant changes complimentary to the ISNCSCI and SCIM-SS assessments. 


\section{Introduction}

After cervical spinal cord injury (SCl), motor and sensory impairments cause limitations in upper limb function which affect performance of activities of daily living (ADLs), independence and, ultimately, restrict participation and quality of life. Previous studies have shown that individuals with tetraplegia consider improvements in upper limb function to be one of the most significant factors in improving quality of life..$^{1,2}$ Longitudinal studies in acute $\mathrm{SCl}$ reveal that most recovery occurs within the first months after cervical $\mathrm{SCl}^{3,4}$ even though the degree of upper limb functional recovery is highly variable. ${ }^{5,6}$ Nevertheless, clinical recovery assumes rather complex dimensions that are not sufficiently appreciated by a single outcome measure (such as motor scores) but stem from multiple factors following acute tetraplegia. The International Classification of Functioning, Disability and Health $(\mathrm{ICF})^{7}$ provides a comprehensive framework to improve the appreciation of clinical recovery.

Several upper limb outcome measures are available, however only a few have been specifically developed for $\mathrm{SCl}^{8,9}$ and these have limited psychometric properties. ${ }^{9} \mathrm{At}$ present, the International Standards of Neurological Classification of Spinal Cord Injury ${ }^{10}$ (ISNCSCI) is the current standard to assess neurological recovery and the Spinal Cord Independence Measure ${ }^{11}$ (SCIM) is the most widely used outcome measure to document change in ADLs in individuals with SCI. A tetraplegia-specific outcome measure, the Graded and Redefined Assessment of Strength, Sensibility and Prehension (GRASSP) was developed ${ }^{12}$ in an attempt to demonstrate how changes in impairment (i.e. neurological deficit which falls into the ICF component 'body structure and body function') may change over time and contribute to complex upper limb tasks, which refer to the ICF component 'activity and participation'. In individuals with chronic cervical SCI (i.e. more than 6 months post-injury), all subtests within the GRASSP have shown high inter-rater and test-retest reliability (0.84-0.96 and $0.86-0.98$, respectively) and favorable validity ${ }^{13}$ and it is highly predictive of upper limb function and self-care in acute cervical SCI. ${ }^{14}$

Responsiveness of the GRASSP, so far not yet established, may be defined as its sensitivity in detecting changes in upper limb function over time, allowing for the evaluation of patterns of recovery in cervical $\mathrm{SCl}$ during rehabilitation and in the assessment of treatments for SCl. For further evaluation of the clinically relevant changes it is important to also include the clinician's perception of change in upper limb function and daily functioning. The latter aims to capture clinical judgments reflecting the degree of changes in the patients' life beyond the changes as measured by a standardized clinical assessment tool. ${ }^{15}$

This prospective study in acute cervical $\mathrm{SCI}$ up to 1 year post injury thus aimed to investigate: (1) the responsiveness of the GRASSP subtests; (2) the responsiveness of the 
GRASSP subtests compared and related to ISNCSCI and the spinal cord independence self-care subscore (SCIM-SS); (3) the clinical appreciation of changes in GRASSP and SCIM-SS by using a clinician-rated outcome measure (CROM), and (4) recovery profiles in GRASSP strength and prehension.

\section{Methods}

\section{Study design}

The study was designed as a prospective longitudinal multicenter study.

\section{Study population}

Participants were recruited from five European SCI centers specializing in the rehabilitation of individuals with SCI. Participants were recruited between January 2009 and June 2011. Inclusion criteria consisted of traumatic or non-traumatic tetraplegia, enrollment within 0 to 10 days post injury and assessment of the American Spinal Injury Association (ASIA) Impairment Scale (AIS grade of A, B, C, or D) ${ }^{10}$ Individuals were included if their injury level was between $\mathrm{C} 3$ and $\mathrm{T} 1$ in the case of AIS A patients and C1-T1 in those with incomplete injuries. Excluded were those individuals with any accompanying severe neurological (e.g., traumatic brain injury) or medical disorders or those aged less than 16 years. Participants were recruited after providing written informed consent. The study was approved by the relevant local ethics committees.

\section{Procedures}

Assessors who had at least one year experience in working with individuals with $\mathrm{SCl}$ were trained to ensure high-quality examinations and to reduce inter-observer variability. Occupational therapists performed the GRASSP and rated the questionnaires. For organizational reasons, it was unavoidable that, in some cases, the two assessments were performed by the same therapist. Physicians performed the ASIA testing and the SCIM III was completed by physical therapists, nurses and occupational therapists. The GRASSP takes between 30-45 minutes to complete.

The assessments and clinical examination were performed during inpatient rehabilitation between 0-10 days, at 1 month (range 16-40 days), 3 months (range 70-98 days), 6 months (range 150-186 days) after cervical SCI and in outpatient clinics at 12 months (range 300-400 days) after cervical SCI. The clinician-reported outcome measure 
(CROM) were performed at 3, 6 and 12 months post-injury. The AIS classifications were calculated by a computer algorithm, ${ }^{16}$ according to the definitions in the International Standards. ${ }^{10}$

\section{Outcome measures}

We have included a table (Table 3.1) to explain the acronyms of outcome measures used in this study.

The GRASSP is a three-domain, upper limb clinical outcome measure for individuals with tetraplegia, contains five subtests and measures each upper limb separately. The subtests within GRASSP are:

Manual muscle testing ${ }^{17}$ (MMT). Ten muscles in the arm and hand were assessed on both sides. Each item (muscle) was scored between 0 and 5 , whereby score 5 represented normal strength and score 0 total paralysis. The total score for both sides is the sum of all item scores with a maximum of 100 .

Semmes and Weinstein Monofilament (SWM). The touch threshold was assessed using the pocket version of $\mathrm{SWM}^{18}$ (North Coast Medical, Inc, Campbell, CA), with four probes (monofilaments) across three dorsal and three palmar locations for each hand as described in the instructions of the SWM mini-kit ${ }^{18}$ and the GRASSP manual. The pressure applied and sensation elicited was represented by numeric values ranging from

Table 3.1 Summary of outcome measures

\begin{tabular}{cl}
\hline Abbreviation & Outcome measure name \\
\hline GRASSP & Graded Redefined Assessment of Strength, Sensibility and Prehension \\
MMT & Manual muscle testing \\
SWM & Semmes and Weinstein monofilament \\
SWMP & Semmes and Weinstein monofilament; palmar \\
SWMD & Semmes and Weinstein monofilament; dorsal \\
QIG & Qualitative grasping \\
QtG & Quantitative grasping \\
ISNCSCI & International Standards for Neurological Classification of Spinal Cord Injury \\
UEMS & Upper extremity motor score \\
LT & Light touch \\
SCIM & Spinal Cord Independence Measure \\
SCIM-SS & Spinal Cord Independence Measure; self-care subscale \\
CROM & Clinician-rated outcome measure \\
ICF & International Classification of Functioning, Disability and Health \\
\hline
\end{tabular}


0 (no response) to 4 (normal sensation). Three locations for the dorsal (SWMD) or palmar (SWMP) side are summed as subtest total score for both sides, ranging from 0 to 24 points.

Qualitative Grasping (Q/G). Three grasps were assessed according to the grasp acquisition outlined in the GRASSP manual and developed by the GRASSP International Research and Design Team. The grasps were scored between 0 (no voluntary control of wrist and digits when grasping) and 4 (normal voluntary control of wrist and digits when generating the grasp). The sum of the grasping quality subtest scores for both upper limbs ranges from 0 to 24 points.

Quantitative Grasping (QtG). In a strictly standardized way, six prehension tasks, such as picking up a key from a table, were performed for each arm separately, as adapted from the Sollerman Hand Function Test. ${ }^{19}$ Each task was scored on a 0 to 5 scale according to the grasp used. Details of scoring are available in the GRASSP manual. The total score was the sum of all task scores with a range of 0 to 60 for both sides.

The SCIM is a global measure of fundamental daily activities specific to individuals with $\mathrm{SCI}$ and focuses on gained independence. ${ }^{11}$ The SCIM III has well-validated psychometric properties ${ }^{20-22}$ and the SCIM-SS reflects upper limb performance. ${ }^{23}$ In our study, the SCIM III-SS was therefore selected. The sum of the SCIM III-SS ranges between 0 and 20.

The CROM was developed by the GRASSP International Research and Design Team and consists of four questions regarding perceived physical change over time. The questions of the CROM were rated by occupational therapists, based on their perceived impressions of the change in performance of patients' (1) strength; (2) sensation; (3) fine motor tasks (ability to perform tasks such as screwing the cap from a toothpaste tube) and (4) functional tasks (ability to perform tasks such as eating independently, holding a cup and brushing one's teeth) between 1 and 3 months, between 3 and 6 months and between 6 and 12 months post-cervical SCI. The scale of the CROM has seven categories ranging from 1 (much worse) to 7 (much better).

The neurological assessment was performed according to the ISNCSCI protocol. ${ }^{10}$ The ISNCSCI was selected to classify the neurological level of injury (NLI) and the overall AIS grade. The ISNCSCI UEMS and the ISNCSCI LT (C6-C8) were used in this study. Five key muscle groups of the upper limb in both arms were assessed and scored between 0 (total paralysis) and 5 (normal strength). The sum value of this score ranges from 0 and 50 for both sides. Sensation (LT) in three dermatomes (C6-C8) were scored as follows: 0 (absent sensation); 1 (impaired sensation) and 2 (normal sensation). The sum value of this score ranges from 0 to 12 points for both sides. 


\section{Data analysis}

Descriptive statistics were used to determine the frequency, mean, standard deviation and range of the study participants' characteristics including AIS grade, lesion level, sex and age. Parameters were visually checked for normal distribution by a $Q Q$ plot.

There are many approaches for assessing responsiveness but no general consensus has yet been reached on the best method to use..$^{24,25}$ Internal responsiveness is defined as the ability of a measure to change over a particular, specified time period. ${ }^{25}$ External responsiveness reflects the extent to which changes in a measure over a specified time relate to corresponding changes in referential measurement of health status. ${ }^{25}$ Both internal and external responsiveness have been used in our comparisons.

Because differences in recovery and responsiveness depending on completeness of the lesion can be expected, we performed additional subgroup analyses (motor complete AIS: A-B and motor incomplete AIS: C-D).

\section{Internal responsiveness}

For internal responsiveness, a linear mixed model was used to assess change of the measure controlling for time. The level of significance was set at $\alpha<0.05$.

Paired t-tests (Bonferroni adjusted) based on the linear mixed model were performed to localize significant differences between time intervals. The following time intervals were selected: 3,6 , and 12 months to 1 month; 6 and 12 to 3 months and lastly 12 months to 6 months. $95 \%$ confidence intervals $(\mathrm{Cl})$ of changes between time intervals were calculated. The standardized response mean (SRM) is now widely used ${ }^{26}$ to express effect size and was calculated for the six intervals mentioned above. SRM is the mean delta score between the above defined intervals divided by the standard deviation (SD) of the delta score. ${ }^{26-28}$ Values of 0.20 indicate low responsiveness; 0.50 medium responsiveness; and above 0.80 , high responsiveness. ${ }^{29}$

\section{External responsiveness}

The external responsiveness of the GRASSP was quantified using correlation analysis and receiver operating characteristics (ROC) analysis. The level of significance was set at $\alpha<0.05$. UEMS, LT (C6-C8) and SCIM III-SS were used as reference measures of GRASSP and CROM was used as an external standard for GRASSP.

Correlation analysis was performed between subtests of GRASSP and the reference measures using Spearman's rho correlation coefficient to determine the relationship 
between them. Correlations in the range of 0 to 0.25 were interpreted as none to poor, 0.26 to 0.50 as fair, 0.51 to 0.75 as moderate to good, and 0.76 to 1.0 as very good to excellent. ${ }^{29}$ With respect to aim 3, ROC analysis was performed to analyse the area under the curve (AUC) of MMT, SWM, QtG and SCIM III-SS delta values, in order to discriminate between patients with and without improvement as rated by clinicians (CROM). Values of the AUC between 0.7 and 1.00 indicate acceptable to excellent discrimination. ${ }^{30}$ For $\mathrm{ROC}$ analysis we dichotomized the four CROM questions strength, sensation, fine motor tasks and functional tasks to assign individuals into an improved or non-improved group. Individuals were allocated to the improved group if the reported outcomes were: 'somewhat better', 'better' or 'much better'. All participants with: the 'same', 'somewhat worse', 'worse' or 'much worse' reported outcomes were allocated to the non-improved group. Furthermore we calculated mean delta scores for MMT, SWM, QtG and SCIM III-SS between the assessment stages (1-3 months, 3-6 months and 6-12 months).

\section{Recovery profile}

The annualized rate of recovery was calculated by dividing the amount of recovery between two assessments by the time interval between the two assessments. This value was then multiplied by 365 to express the rate of recovery during a particular interval that would have been expected if it were to have continued over 1 year, as used by Waters et al. . $^{3,4}$ All data were analyzed using SPSS version 18.0 for Windows.

\section{Results}

Of the total 74 participants included, 69 had a traumatic and 5 a non-traumatic SCI (two ischemic in origin and three cases of spinal canal stenosis). Some GRASSP data were missing for 14 participants at the 6 months assessment and for 15 participants at the 12 months assessment (due to medical $(n=2)$ and logistical reasons (e.g. discharge, $\mathrm{n}=12)$ ). Because of the lower number of participants between 0-10 days post-injury ( $n=40$ ) we excluded this baseline time point from our analyses. Data of all parameters were approximately normally distributed. There was no statistically significant difference between the sum scores of the right and left sides and, therefore, all analyses were made for the sum score of the right and left sides combined. Detailed cohort characteristics are presented in Table 3.2 
Table 3.2 Demographic and clinical characteristics of participants $(n=74)$

\begin{tabular}{|c|c|}
\hline Characteristics & $\mathrm{n}(\%)$ \\
\hline \multicolumn{2}{|l|}{ Cause of $\mathrm{SCl}$} \\
\hline Traumatic & $69(93.2 \%)$ \\
\hline Non-traumatic & $5(6.8 \%)$ \\
\hline \multicolumn{2}{|l|}{ Site } \\
\hline Klinik Hohe Warte Bayreuth (D) & $25(33.8 \%)$ \\
\hline Unfallklinik Murnau (D) & $1(1.4 \%)$ \\
\hline Orthopädische Universitätsklinik Heidelberg (D) & $9(12.2 \%)$ \\
\hline Balgrist University Hospital Zurich $(\mathrm{CH})$ & $14(18.9 \%)$ \\
\hline Swiss Paraplegic Center Nottwil $(\mathrm{CH})$ & $25(33.8 \%)$ \\
\hline \multicolumn{2}{|l|}{ Gender } \\
\hline Female & $23(31.1 \%)$ \\
\hline Male & $51(68.9 \%)$ \\
\hline Age (mean years; SD; min/max) & $49( \pm 18 ; 18-87)$ \\
\hline \multicolumn{2}{|l|}{ AIS } \\
\hline 1 month (range $16-40$ days) $(n=74)$ & $\begin{array}{l}\text { A: } 18(24.3 \%) \text {; B: } 12(16.2 \%) \text {; } \\
\text { C: } 10(13.5 \%) \text {; }: 34(45.9 \%)\end{array}$ \\
\hline 3 months (range $70-98$ days) $(n=68)$ & $\begin{array}{l}\text { A: } 13(17.6 \%) \text {; }: 10(13.5 \%) \text {; } \\
\text { C: } 8(10.8 \%) \text {; }: 37(50.0 \%)\end{array}$ \\
\hline 6 months (range $150-186$ days) $(n=60)$ & $\begin{array}{l}\text { A: } 14(18.9 \%) ; \text { B: } 7(9.5 \%) \\
\text { C: } 4(5.4 \%) ; \text { D: } 35(47.3 \%)\end{array}$ \\
\hline 12 months (range $300-400$ days) $(n=58)$ & $\begin{array}{l}\text { A: } 10(13.5 \%) ; B: 6(8.1 \%) ; \\
\text { C: } 6(8.1 \%) ; D: 36(48.5 \%)\end{array}$ \\
\hline \multicolumn{2}{|l|}{ Neurological level at 1 month } \\
\hline C1 & $4(5.4 \%)$ \\
\hline $\mathrm{C} 2$ & $6(8.1 \%)$ \\
\hline C3 & $11(14.4 \%)$ \\
\hline $\mathrm{C} 4$ & $26(35.1 \%)$ \\
\hline C5 & $17(23.0 \%)$ \\
\hline C6 & $7(9.5 \%)$ \\
\hline $\mathrm{C} 7$ & $1(1.4 \%)$ \\
\hline C8 & $1(1.4 \%)$ \\
\hline T1 & $1(1.4 \%)$ \\
\hline
\end{tabular}

Abbreviations: $\mathrm{n}$, sample size; $\mathrm{SCl}$, spinal cord injury; D, Germany; $\mathrm{CH}$, Switzerland; AIS, American Spinal Injury Association Impairment Scale; C2, cervical dermatome 2; C, cervical T1, thoracic dermatome 1; T, thoracic; dermatomes are indicated by numbers; SD, standard deviation.

\section{Internal responsiveness GRASSP}

Linear mixed model analysis showed that overall and in both subgroups, the GRASSP subtest mean scores MMT, SMW, SWMP, SWMD, QIG and QtG differed significantly over time $(p<0.0001)$. 
Pairwise comparison showed that MMT mean score significantly improved over all time intervals, both overall and for both subgroups with exception of the AIS C-D group, in which no significant change between 6-12 months was seen. Overall and in both subgroups, SWM, SWMP, SWMD, QIG and QtG mean scores significantly improved from 1 month to 12 months, but no significant difference was found between 3-6 months and 6-12 months.

Overall and in both subgroups, the SRM for MMT was large between all intervals except for the entire group and AIS C-D group, where a moderate SRM between 6-12 months was found. For QIG, moderate-to-large responsiveness was found from 1 month to 12 months and between 3-6 months in the group as a whole and in the AIS C-D subgroup. A moderate-to-large responsiveness was observed for QtG overall and in both subgroups over all time intervals except for the AIS A-B group between 3-6 months. The SWM tests showed poorer internal responsiveness compared to the other GRASSP subtests results. Detailed results of the pairwise comparison and the SRM of all time intervals for GRASSP subtests and the different groups are presented in Table 3.3.

Internal responsiveness of GRASSP compared to internal responsiveness of the reference measures

The results of the linear mixed model showed that the UEMS, LT (C6-C8) and SCIM-SS mean scores differed significantly over time $(p<0.0001)$.

As shown in Table 3.3, the GRASSP subtests showed similar, significant differences over the same time intervals as the scores of the UEMS and SCIM-SS (Table 3.4) with exception of LT (C6-C8), where no significant differences between time intervals was found and UEMS, in which no significant difference between 6-12 months was observed.

Both overall and for both subgroups, the SRM values of the GRASSP subtests (Table 3.3) were higher compared to the reference measures (Table 3.4 and Figure 3.1) with the exception of SCIM-SS. Detailed results of the pairwise comparison and the SRM of all time intervals for the reference measures and the different groups are available in Table 3.4. Figure 3.1 shows the SRM up to 12 months for the GRASSP subtests compared to the reference measures (as visual complement with Table 3.3 and Table 3.4). 

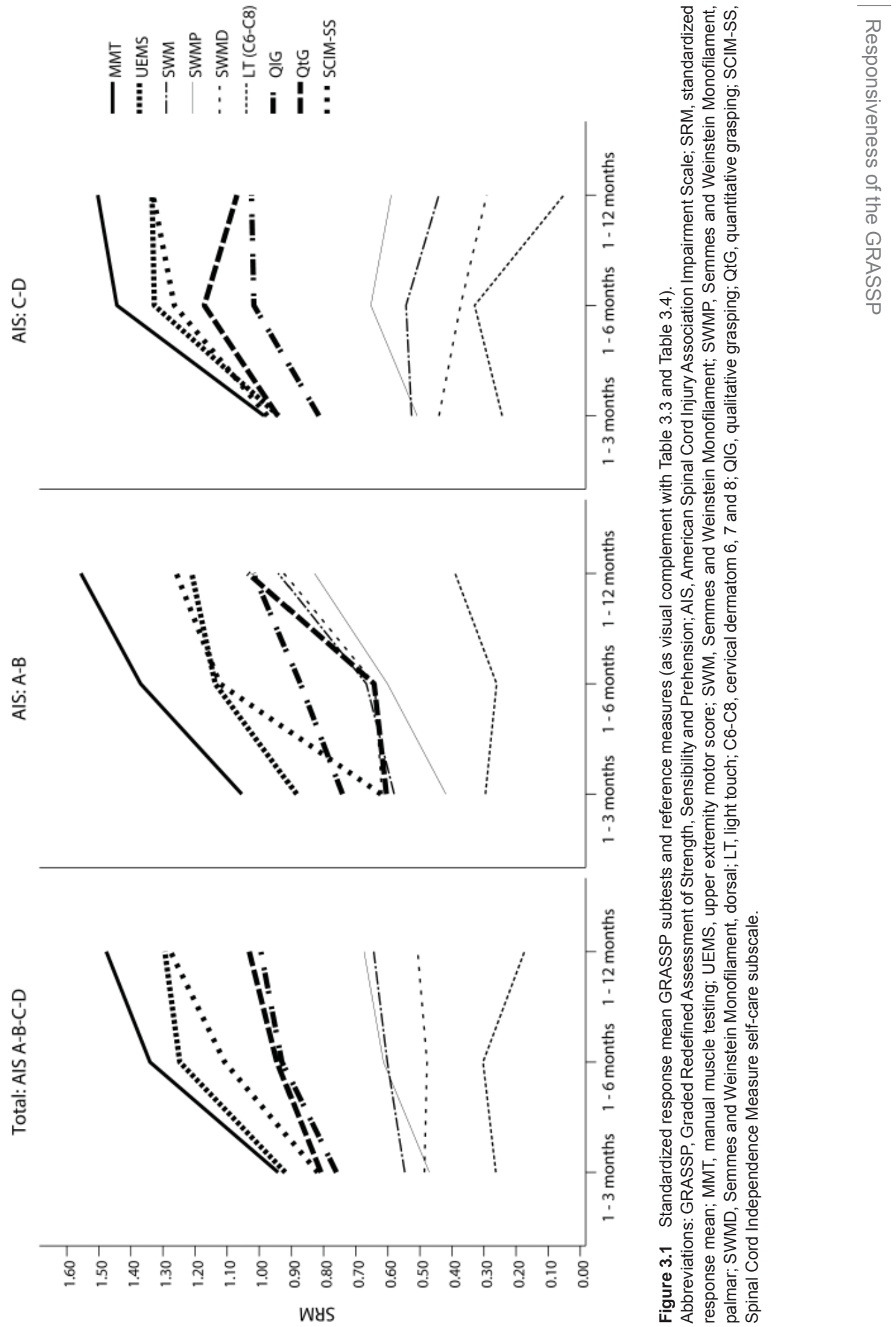


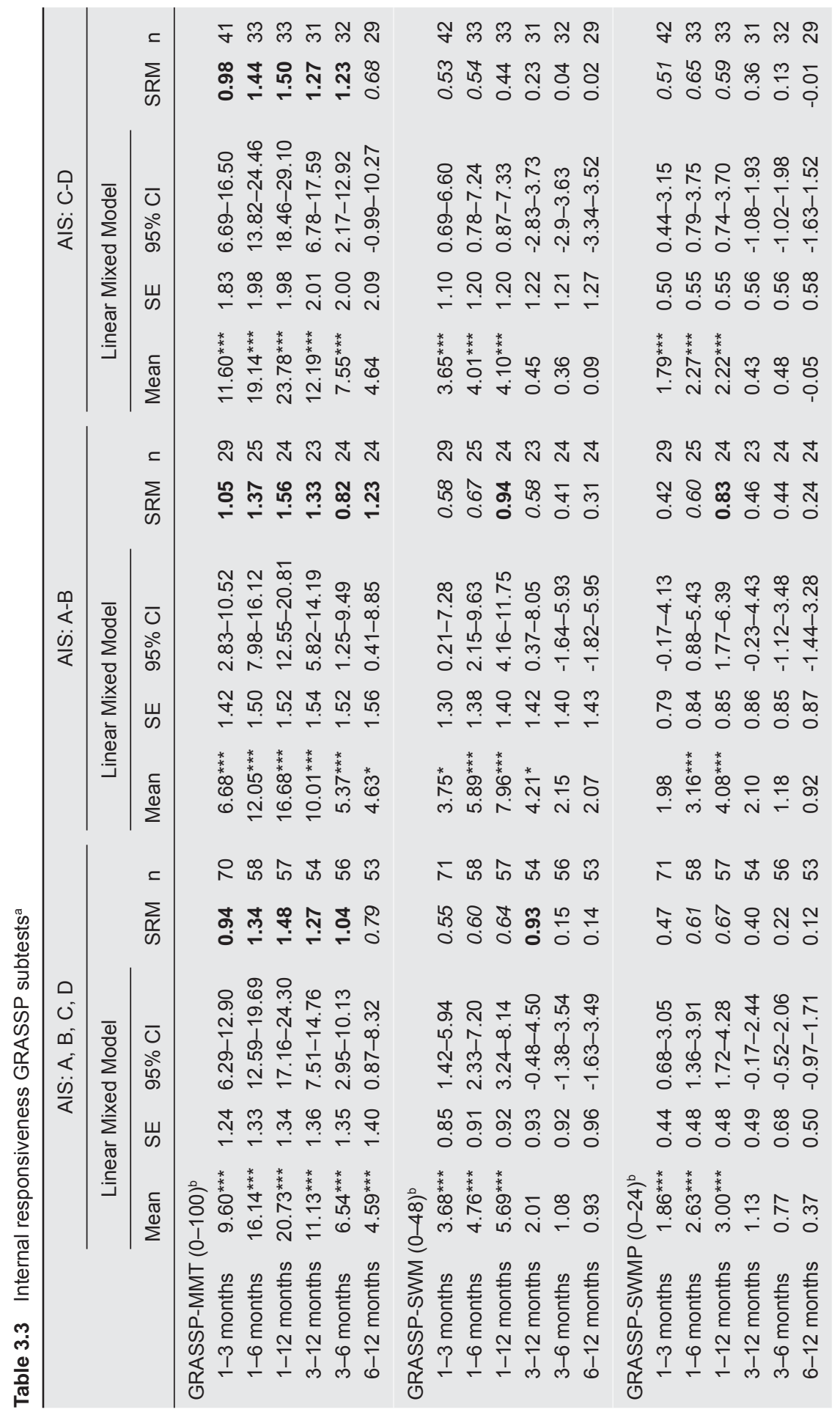




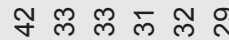

Ұ

० 0000

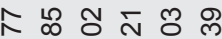
๓ め

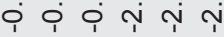

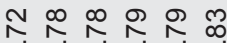
० 00000

๘ $\leftarrow+\frac{1}{-} \dot{0}$

ㅇํㄴ 논

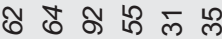
० 00000

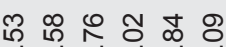
\% ए $\begin{array}{cccc}0 & 0 \\ 0 & 0 & 0 & 0 \\ 0 & 0 & 0 & 0\end{array}$

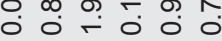
융용우둥요 유 OOOOO

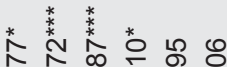
-

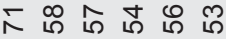

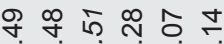
० 00000

느는융요 ए के ช. 잉

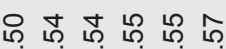
० 00000

武絭喜类品的品

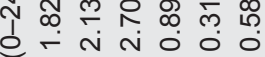

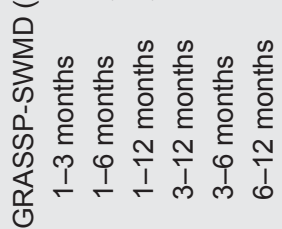

ఫ

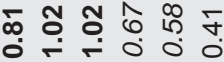

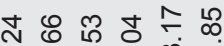
फ $\varphi$ 个

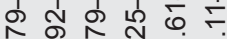

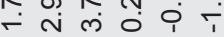

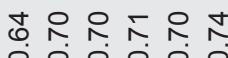
००००00

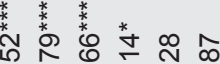
mं

ㄱํㄴ र 0 O 0 . 000 œ 요 ๙ กับ

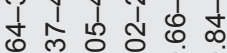
○

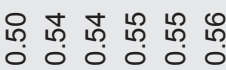

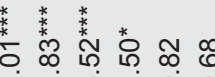
서ํ

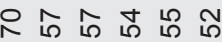
ำร 月 \& ิำ 00000 8 \% $\%$ \& 8

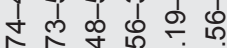

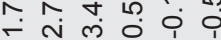

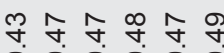
$\circ 00000$ - மं ம

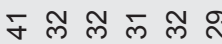

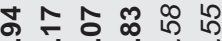
० ஜ ஷ เి

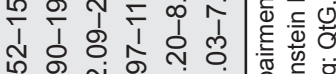
ט

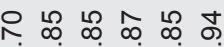

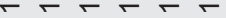

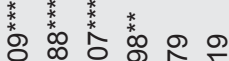
F芒的

ผ กิ กิ สิก ㅇํㅇㅇㅛ 웅 $00 \mathrm{H}, \mathrm{A} 0$

๓ $\stackrel{0}{2} \stackrel{\infty}{\infty}$ के

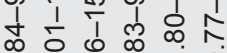

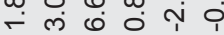
守 $\overline{0} 0$ 운

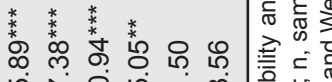
ம $\sim$ 은

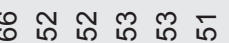

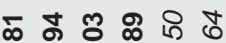
० ०

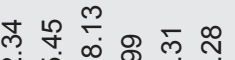
ㄴ.

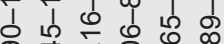
它

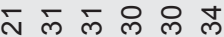
సৃ

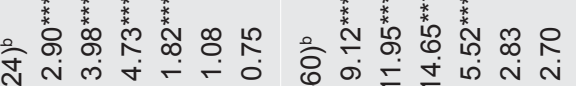
o O कि है है ह $\stackrel{\varepsilon}{\sim}$ ह ह ह

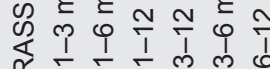

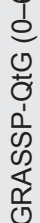

曾

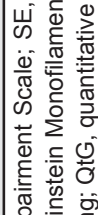
हू $\sum_{3}^{\circ}$ के 들 든 क ल की 


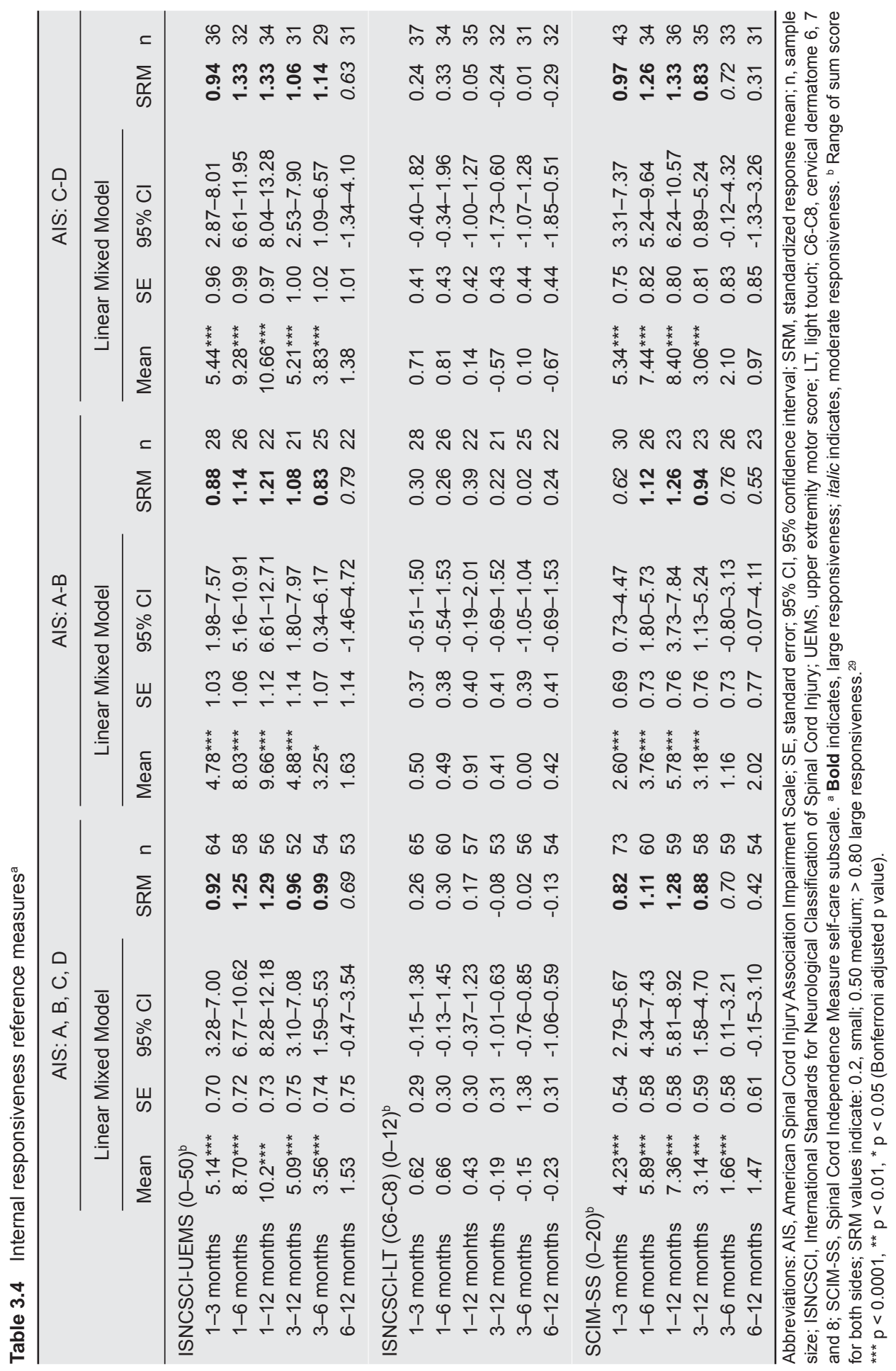




\section{External responsiveness}

The Spearman correlations between GRASSP subtests MMT, SWM and QtG with the reference measures at 1, 3, 6 and 12 months were moderate to high. Detailed results of the correlation analysis are presented in Table 3.5.

ROC analyses showed that the AUC value for MMT, SWM, QtG and SCIM-SS were acceptable to excellent (ranging from 0.68 to $0.87, p<0.05$ to $p<0.001$ ) at $1,3,6$ and 12 months post-injury. Detailed results for ROC analysis can be found in Table 3.6 and Figure 3.2 (as visual complement with Table 3.6).

\section{Recovery profile}

The overall, annualized motor and prehension recovery rate showed a comparable course in the AIS A-B and AIS C-D subgroups (Figure 3.4) although in the AIS C-D subgroup individuals had higher scores at the beginning of rehabilitation (Figure 3.3 and Figure 3.4). A very high motor and prehension recovery rate between 1-3 months after injury was achieved in both subgroups. After 3 months, this rate rapidly declined (QtG more steeply than MMT) and between 6-12 months the motor and prehension recovery rate was very low, although MMT and QtG continued to show improvement up to 12 months in both subgroups. For detailed results, see Figure 3.4.

GRASSP-MMT (3-6 months)

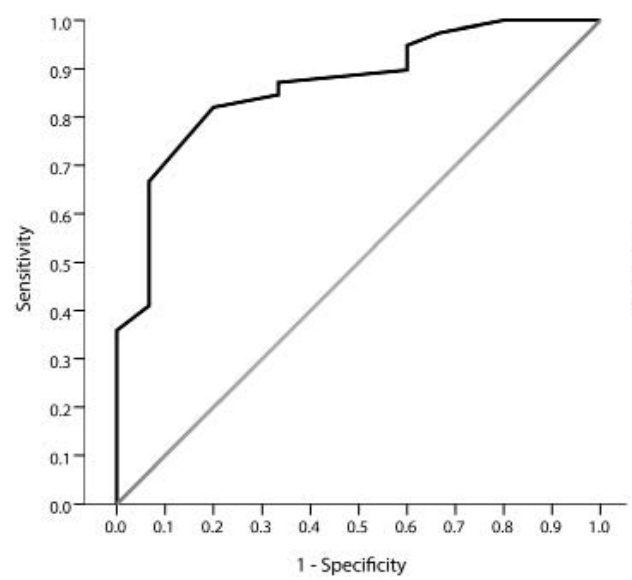

GRASSP-QtG (3-6 months)

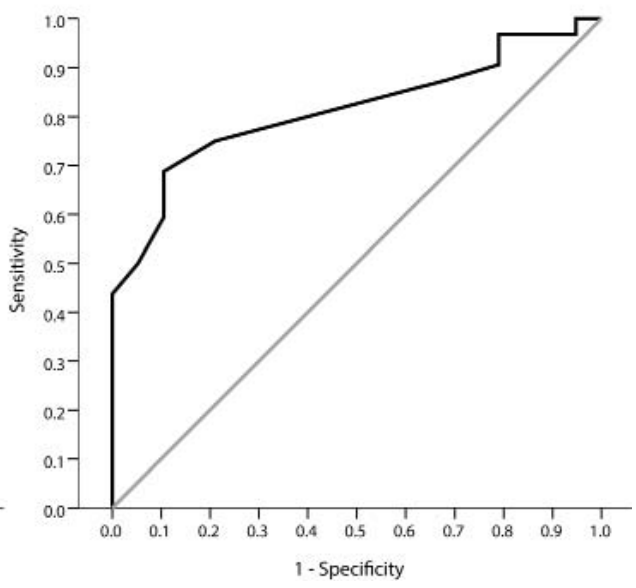

Figure 3.2 Receiver operating characteristics (ROC) curves.

The figures show the GRASSP-MMT and GRASSP-QtG ROC curves between patients with and without improvement as rated by clinicians at 6 months compared to 3 months (as visual complement with Table 3.6). The diagonal represents the line of no discrimination. Points above this line indicate good classification results. The bigger the area under the ROC curve, the better the accuracy between GRASSP changes and clinicians' ratings.

Abbreviations: GRASSP, Graded and Redefined Assessment of Strength, Sensibility and Prehension; MMT, manual muscle testing; QtG, quantitative grasping. 


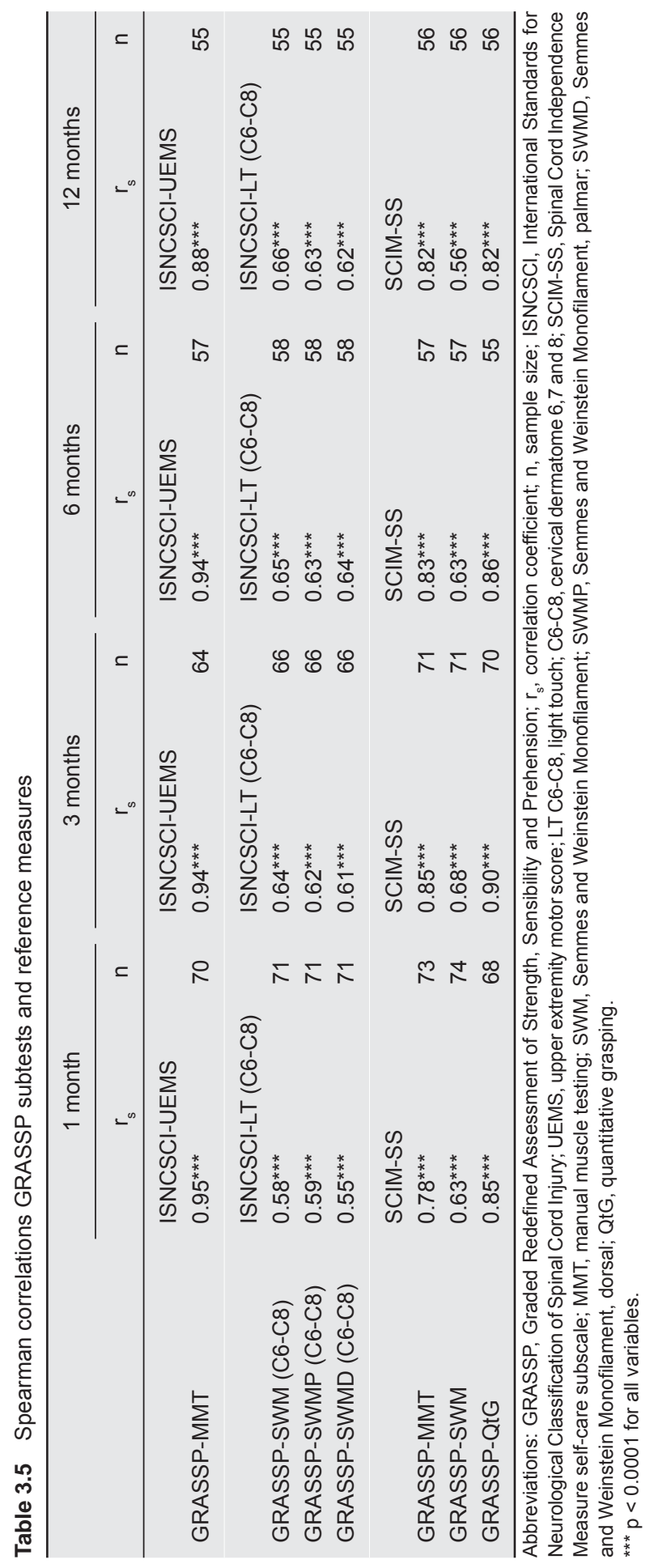




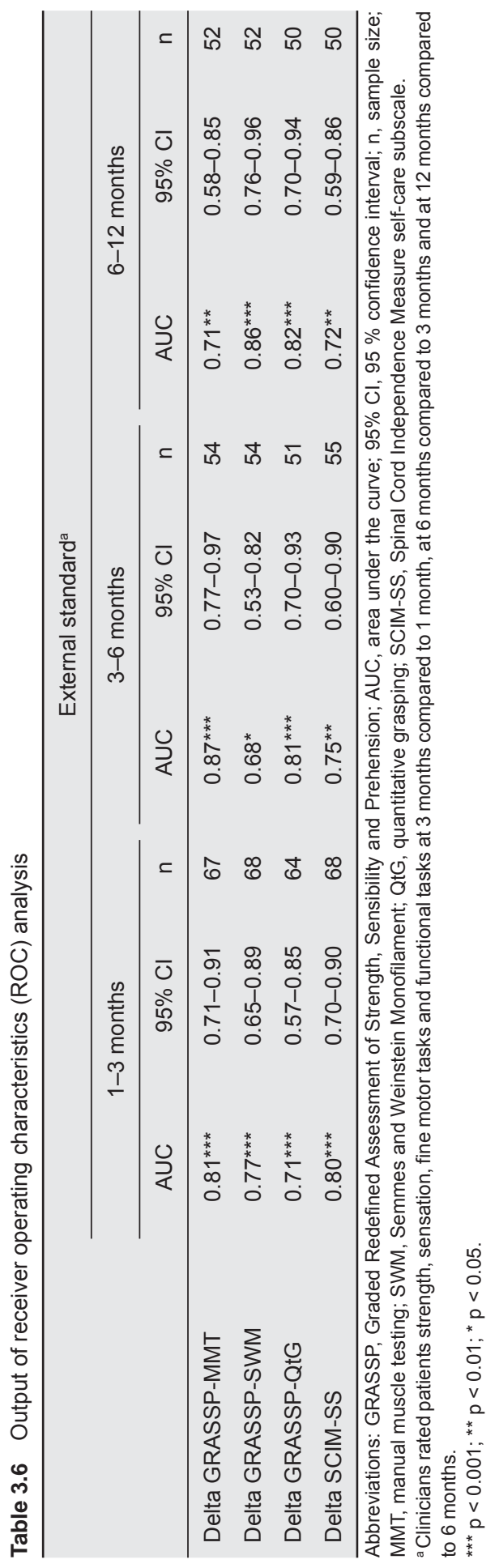



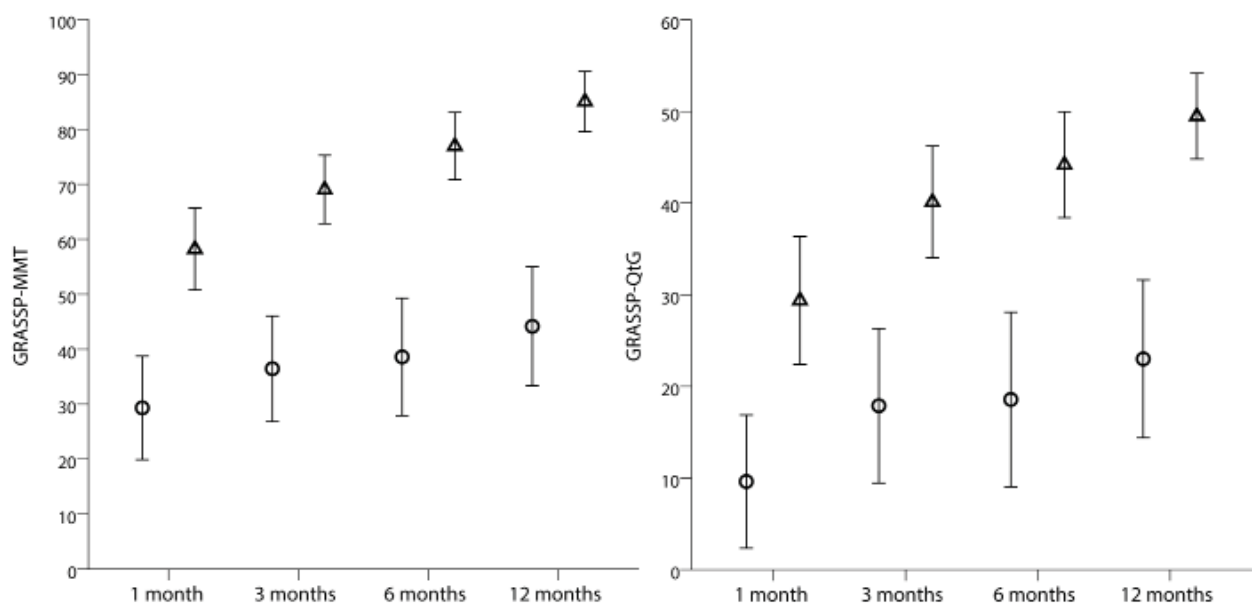

Figure 3.3 GRASSP-MMT and GRASSP-QtG recovery over time.

Indicated are the mean and $95 \%$ confidence interval from 1 month to 1 year after cervical spinal cord injury. Abbreviations: GRASSP, Graded and Redefined Assessment of Strength, Sensibility and Prehension; MMT, manual muscle testing; QtG, quantitative grasping; AIS, American Spinal Cord Injury Association Impairment Scale. o, AIS: A-B, $\Delta$, AIS: C-D.
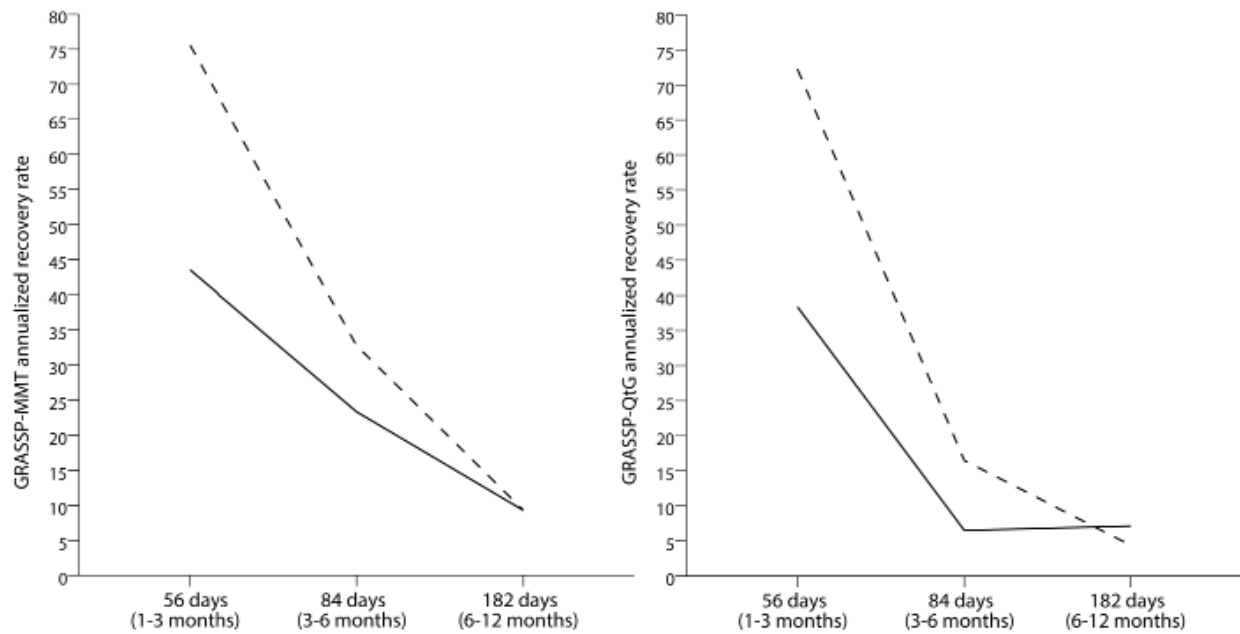

Figure 3.4 GRASSP-MMT and GRASSP-QtG annualized recovery rate.

The figures show the MMT and QtG recovery rate at 56, 84 and 182 days after cervical spinal cord injury. The motor (MMT) and functional (QtG) recovery rate was calculated by dividing the amount of recovery observed between two assessments by the time interval in between the two assessments and by converting this value to change per year. This value indicates the recovery rate, assuming it had remained constant over 1 year. Abbreviations: GRASSP, Graded and Redefined Assessment of Strength, Sensibility and Prehension; MMT, manual muscle testing; QtG, quantitative grasping; —_, AIS: A-B; - - - - - AIS: C-D. 


\section{Discussion}

This study showed the excellent internal and external responsiveness of the GRASSP during the first year after cervical $\mathrm{SCl}$ and provided distinct motor and prehension recovery profiles. More importantly, the GRASSP is complementary to clinical assessment using ISNCSCI standards due to its higher responsiveness. Furthermore, improvements as indicated by the GRASSP were rated as clinically meaningful changes according the clinicians' impressions of patients' recoveries. These findings suggest that the GRASSP can provide meaningful information for clinical trials beyond the ISNCSCI standards.

\section{Responsiveness}

All GRASSP subtests showed good internal responsiveness over time.

MMT was the most responsive GRASSP subtest with even higher, significant changes seen in the AIS A-B group between 6-12 months than in the AIS C-D group.

MMT was more responsive than UEMS throughout, likely due to the expanded combination of muscle groups. MMT, as defined in the GRASSP, incorporated additional distal (extensor digitorum, opponens pollicis, flexor pollicis longus and first dorsal interosseus,) and proximal (anterior deltoid) muscle groups in addition to the standard muscles (elbow flexors, wrist extensors, elbow extensors, finger flexors and small finger abductors) assessed by the ISNCSCI protocol. These additional muscles provide important information regarding innervation and contributed to the higher responsiveness in this study in accordance with findings reported in a recent longitudinal study in acute cervical $\mathrm{SCl}^{14}$ as well as in a cross sectional study in chronic SCl. ${ }^{13}$ Therefore, the assessment of additional arm and hand muscles as defined by the GRASSP provides a more sensitive appreciation of upper limb function and supports the application of the GRASSP as a more accurate assessment tool in cervical SCI than those used previously (ISNCSCI).

MMT in the AIS C-D group between 6-12 months post-injury might show a ceiling effect in detecting further small changes at this stage. For future studies, we recommend the additional assessment of strength using hand-held dynamometry in those individuals with less paretic muscles (minimum MMT score of 3 ) to avoid ceiling effects in individuals with high MMT scores at the beginning of rehabilitation. In general QtG was equally sensitive as the SCIM-SS to changes in cervical SCI but was more responsive between 6-12 months. SCIM-SS provides a clinically meaningful categorization of functioning in ADLs with a focus on gained independence while the QtG stresses the capacity to 
perform standardized movement tasks. Although the QtG score is based on unilateral performance of tasks with no compensatory movements, it reflects similar changes to those detected by the SCIM-SS. The advantage of using GRASSP over other outcome measures such as the Van Lieshout test ${ }^{31,32}$ or Capabilities of Upper Extremity Test, ${ }^{33}$ that tend to address overall arm and hand usage, is that it provides more detailed information on how functional improvements are achieved. Likewise, a functional measure such as the SCIM III is not designed to establish the neurological state of an individual when performing tasks or whether those tasks are performed bimanually or with compensatory movements. In contrast, the subtest scores of GRASSP are specifically designed to disentangle detailed motor and sensory functions contributing to the outcome of upper limb function. The detailed assessment reveals how changes in function are related to neurological improvements following spinal cord injury, something which cannot be elucidated by the SCIM III. In addition, the GRASSP subtests also include standardized prehension tests that are related to changes in neurological outcomes. These combined assessments permit the determination of whether changes in function are based on improvement through compensatory movements or on improvement of neurological function. Accordingly, QtG provides a detailed scoring of standardized tasks focusing on the form of the grasp and therefore is able to explain how changes up to 1 year post-injury are achieved.

Sensory changes were rather limited as assessed by the SWM. However, SWM was significant and more sensitive to small gains between 1-3 months, something not found with $L T$ (C6-C8). The present results suggest that SWM is sensitive to minor impairments, which are less detectable by LT testing; findings also confirmed in cross-sectional studies by Kalsi-Ryan et al. and Velstra et al. ${ }^{13,34}$ These authors observed greater sensitivity of the SWM in individuals with acute and chronic SCI compared with the values reported when using LT.

Likely due to the broader scaling of the SWM (by applying different sensory modalities) as well as the additional palmar test locations, more changes in sensation were detected with SWM than with LT.

All GRASSP subtests revealed significant, moderate-to-excellent correlations with the established reference measures at each time point (external responsiveness). These findings support previously published data that showed significant and moderate-to-strong correlations of MMT, SWM, QtG or cervical motor levels with self-care in longitudinal as well as cross-sectional studies in acute and chronic cervical SCI.5,6,13,14,23,35 The large 
SRMs, particularly for MMT and QtG, reflect great clinical significance ${ }^{25,36,37}$ which was supported by the clinicians' ratings (external standard), used as an indicator for clinically meaningful change. The results showed large AUC, indicating that changes in GRASSP subtests and SCIM-SS were rated as clinically meaningful in accordance with the external standard (external responsiveness). ${ }^{25,28,38,39}$

\section{Recovery profiles}

It was expected that strength (MMT) and prehension (QtG) would mostly improve within the first 3 months of injury where improvements in general functional skills and motor recovery are most prominent compared to later stages of rehabilitation. ${ }^{3-5,40-45}$ In contrast the present study revealed significant improvements in strength between all timepoints up to 12 months post-injury in the entire group and A-B subgroup and up to 6 months post-injury in the C-D subgroup.

The prehension $(\mathrm{QtG})$ and motor (MMT) recovery rate are comparable with the annualized recovery rate reported elsewhere..$^{3,4,44,45}$ This rate decreased with time after cervical SCI. Between 1-3 months post-injury, individuals with AIS C-D showed a motor and prehension recovery rate almost twice as high as that in individuals in the AIS A-B subgroup. From 3 months to 1 year after injury, the motor and prehension recovery rate declined rapidly, although motor changes were larger compared to prehension changes and reached a rate similar in both subgroups at 6-12 months. Strength and prehension still showed improvement up to 12 months in both subgroups, which is a new finding.

\section{Implications for rehabilitation and clinical trials}

This study provides data to assist clinicians and researchers on the value of the GRASSP in acute tetraplegia. We found that the responsiveness of GRASSP is excellent and it is applicable as a primary outcome measure in rehabilitation. Specifically MMT and QtG seem to be most valuable for clinical trials as they are strongly responsive to change over the course of recovery and identify clinically meaningful changes complementary to ISNCSCI and SCIM. Measures with greater responsiveness indices provide greater study power, thereby allowing a study to be completed with fewer individuals. ${ }^{46}$

The observed GRASSP subtest changes were also in accordance with the clinicians' impressions of patient changes, which is a novel finding. Therefore, CROM may be useful in clinical trials to incorporate a clinical judgment that references past experiences to benchmark the progress of a patient. ${ }^{47}$ Experienced clinicians, such as those involved 
in this study, have a good understanding of neurological impairment and functional performance. However, novel questionnaires like the CROM have to be interpreted with caution as they may be influenced by other factors (e.g. clinical judgment, past experience, beliefs regarding treatment effectiveness etc.). Systematic bias in our results cannot be entirely excluded as some assessments of GRASSP and CROM in individual patients were performed by the same therapist. Depending on the study design and research question, it is of course advisable that independent clinicians perform the GRASSP and CROM, thereby minimizing examiner bias.

\section{Limitations}

The effect of different baseline levels of lesion was not assessed in this investigation, but consideration of the amount of change for such patients should be investigated with a higher sample size in future studies.

\section{Conclusion}

The GRASSP is a responsive and clinically meaningful tool for the evaluation of upper limb outcomes in cervical SCl and can be recommended for follow-up assessments. The combined assessment of neurological (body structure and body function) and functional outcomes, e.g. prehension (activity and participation), focused on segmental cervical spinal cord functions that are closely related to other standard assessment tools (ISNCSCI and SCIM) supports the use of GRASSP in the assessment of rehabilitation as well as in interventional clinical trials that seek to detect both subtle and clinically meaningful changes.

\section{Acknowledgments}

We thank the International GRASSP Research and Design Team: Armin Curt, Susan Duff, Michael Fehlings, Sukhvinder Kalsi-Ryan, Claudia Rudhe, and Mary C. Verrier. We are grateful to the individuals with $\mathrm{SCI}$ who participated in this study and the occupational therapists at the participating centers: Spinal Cord Injury Center, Hohe Warte, Bayreuth, Germany; Orthopädische Universitätsklinik, Heidelberg, Germany; Unfallklinik Murnau, Germany; Balgrist University Hospital, Zurich, Switzerland; and the Swiss Paraplegic Center, Nottwil, Switzerland. Furthermore, we thank Dr Aurelio Tobón (Nottwil) for performing the clinical neurological examinations, Mr Tim Killeen (Balgrist) for critically reviewing the article and his invaluable assistance with English language editing, and Dr Doris Maier (Murnau) and Dr Rüdiger Rupp (Heidelberg) for their collaboration on this project. 


\section{Funding}

The authors disclosed receipt of the following financial support for the research, authorship, and/or publication of this article: The study was supported by the Swiss Paraplegic Center, Nottwil, Switzerland, and funded by the NeuroRehab Clinical Research Priority Program at the University of Zurich, Switzerland.

\section{References}

1. Anderson KD. Targeting recovery: priorities of the spinal cord-injured population. J Neurotrauma 2004:21:1371-1383.

2. Snoek GJ, MJ IJ, Hermens HJ, Maxwell D, Biering-Sorensen F. Survey of the needs of patients with spinal cord injury: impact and priority for improvement in hand function in tetraplegics. Spinal Cord 2004;42:526-532.

3. Waters RL, Adkins RH, Yakura JS, Sie I. Motor and sensory recovery following complete tetraplegia. Arch Phys Med Rehabil 1993;74:242-247.

4. Waters RL, Adkins RH, Yakura JS, Sie I. Motor and sensory recovery following incomplete tetraplegia. Arch Phys Med Rehabil 1994;75:306-311.

5. Kramer JL, Lammertse DP, Schubert M, Curt A, Steeves JD. Relationship between motor recovery and independence after sensorimotor-complete cervical spinal cord injury. Neurorehabil Neural Repair 2012;26:1064-1071.

6. Steeves J, Lammertse D, Kramer J, et al. Outcome Measures for Acute/Subacute Cervical Sensorimotor Complete (AIS-A) Spinal Cord Injury During a Phase 2 Clinical Trial. Topics in Spinal Cord Injury Rehabilitation 2012;18:1-14.

7. World Health Organization. International Classification of Functioning, Disability and Health (ICF) [online]. Available at: http://www.who.int/classifications/icf/en/ (Accessed 13.11.2014).

8. van Tuijl JH, Janssen-Potten YJ, Seelen HA. Evaluation of upper extremity motor function tests in tetraplegics. Spinal Cord 2002;40:51-64.

9. Velstra IM, Ballert CS, Cieza A. A systematic literature review of outcome measures for upper extremity function using the international classification of functioning, disability, and health as reference. PM R 2011;3:846-860.

10. Kirshblum SC, Waring W, Biering-Sorensen F, et al. Reference for the 2011 revision of the International Standards for Neurological Classification of Spinal Cord Injury. J Spinal Cord Med 2011;34:547-554.

11. Catz A, Itzkovich M, Agranov E, Ring H, Tamir A. SCIM--spinal cord independence measure: a new disability scale for patients with spinal cord lesions. Spinal Cord 1997;35:850-856.

12. Kalsi-Ryan S, CurtA, Fehlings MG, Verrier MC. Assessment of the Hand in Tetraplegia Using the Graded Redefined Assessment of Strength, Sensibility and Prehension (GRASSP): Impairment Versus Function. Top Spinal Cord Inj Rehabil 2009;14:34-46.

13. Kalsi-Ryan S, Beaton D, Curt A, et al. The Graded Redefined Assessment of Strength Sensibility and Prehension (GRASSP): Reliability and Validity. J Neurotrauma 2012;29:905-914. 
14. Velstra IM, Bolliger M, Tanadini LG, et al. Prediction and Stratification of Upper Limb Function and Self-Care in Acute Cervical Spinal Cord Injury With the Graded Redefined Assessment of Strength, Sensibility, and Prehension (GRASSP). Neurorehabil Neural Repair 2014; 28:632642.

15. Schneider LS, Olin JT. Clinical global impressions in Alzheimer's clinical trials. International psychogeriatrics / IPA 1996;8:277-288; discussion 288-290.

16. Schuld C, Wiese J, Hug A, et al. Computer implementation of the international standards for neurological classification of spinal cord injury for consistent and efficient derivation of its subscores including handling of data from not testable segments. J Neurotrauma 2012;29:453461.

17. Daniels L, Worthingham C. Daniels and Worthingham's Muscle Testing: Techniques of Manual Examination, 6th ed. Washington, 1995.

18. Mackin E, Callahan A, Skiver T, Schneider L, Osterman A. Hunter-Mackin Callahan Rehabilitation of the Hand and Upper Extremity, 5th ed: Mosby: St. Louis, 2003.

19. Sollerman C, Ejeskar A. Sollerman hand function test. A standardised method and its use in tetraplegic patients. Scandinavian journal of plastic and reconstructive surgery and hand surgery / Nordisk plastikkirurgisk forening [and] Nordisk klubb for handkirurgi 1995;29:167-176.

20. Anderson KD, Acuff ME, Arp BG, et al. United States (US) multi-center study to assess the validity and reliability of the Spinal Cord Independence Measure (SCIM III). Spinal Cord 2011;49:880-885.

21. Catz A, Itzkovich M, Tesio L, et al. A multicenter international study on the Spinal Cord Independence Measure, version III: Rasch psychometric validation. Spinal Cord 2007;45:275291.

22. Itzkovich M, Gelernter I, Biering-Sorensen F, et al. The Spinal Cord Independence Measure (SCIM) version III: reliability and validity in a multi-center international study. Disabil Rehabil 2007;29:1926-1933.

23. Rudhe C, van Hedel HJ. Upper extremity function in persons with tetraplegia: relationships between strength, capacity, and the spinal cord independence measure. Neurorehabil Neural Repair 2009;23:413-421.

24. Beaton DE, Bombardier C, Katz JN, et al. Looking for important change/differences in studies of responsiveness. OMERACT MCID Working Group. Outcome Measures in Rheumatology. Minimal Clinically Important Difference. J Rheumatol 2001;28:400-405.

25. Husted JA, Cook RJ, Farewell VT, Gladman DD. Methods for assessing responsiveness: a critical review and recommendations. J Clin Epidemiol 2000;53:459-468.

26. Liang MH, Fossel AH, Larson MG. Comparisons of five health status instruments for orthopedic evaluation. Medical care 1990;28:632-642.

27. Beaton DE, Hogg-Johnson S, Bombardier C. Evaluating changes in health status: reliability and responsiveness of five generic health status measures in workers with musculoskeletal disorders. J Clin Epidemiol 1997;50:79-93.

28. Stratford PW, Binkley JM, Riddle DL. Health status measures: strategies and analytic methods for assessing change scores. Physical therapy 1996;76:1109-1123.

29. Cohen J. Statistical Power Analysis for the Behavioral Sciences. New York, NY: Academic Press Inc., 1977. 
30. Terwee CB, Bot SD, de Boer MR, et al. Quality criteria were proposed for measurement properties of health status questionnaires. J Clin Epidemiol 2007;60:34-42.

31. Spooren Al, Janssen-Potten YJ, Post MW, Kerckhofs E, Nene A, Seelen HA. Measuring change in arm hand skilled performance in persons with a cervical spinal cord injury: responsiveness of the Van Lieshout Test. Spinal Cord 2006;44:772-779.

32. Post MW, Van Lieshout G, Seelen HA, Snoek GJ, ljzerman MJ, Pons C. Measurement properties of the short version of the Van Lieshout test for arm/hand function of persons with tetraplegia after spinal cord injury. Spinal Cord 2006;44:763-771.

33. Marino RJ, Patrick M, Albright W, et al. Development of an objective test of upper-limb function in tetraplegia: the capabilities of upper extremity test. Am J Phys Med Rehabil 2012;91:478486.

34. Velstra IM, Bolliger M, Baumberger M, Rietman JS, Curt A. Epicritic sensation in cervical spinal cord injury: diagnostic gains beyond testing light touch. J Neurotrauma 2013;30:1342-1348.

35. van Hedel $\mathrm{HJ}$, Curt A. Fighting for each segment: estimating the clinical value of cervical and thoracic segments in SCI. J Neurotrauma 2006;23:1621-1631.

36. Guyatt G, Walter S, Norman G. Measuring change over time: assessing the usefulness of evaluative instruments. Journal of chronic diseases 1987;40:171-178.

37. Beaton DE, Bombardier C, Katz JN, Wright JG. A taxonomy for responsiveness. J Clin Epidemiol 2001;54:1204-1217.

38. Deyo RA, Centor RM. Assessing the responsiveness of functional scales to clinical change: an analogy to diagnostic test performance. Journal of chronic diseases 1986;39:897-906.

39. Deyo RA, Diehr P, Patrick DL. Reproducibility and responsiveness of health status measures. Statistics and strategies for evaluation. Controlled clinical trials 1991;12:142S-158S.

40. Burns AS, Ditunno JF. Establishing prognosis and maximizing functional outcomes after spinal cord injury: a review of current and future directions in rehabilitation management. Spine (Phila Pa 1976) 2001;26:S137-145.

41. Yarkony GM, Roth EJ, Heinemann AW, Lovell L, Wu YC. Functional skills after spinal cord injury rehabilitation: three-year longitudinal follow-up. Arch Phys Med Rehabil 1988;69:111114.

42. Ditunno JF, Jr., Cohen ME, Hauck WW, Jackson AB, Sipski ML. Recovery of upper-extremity strength in complete and incomplete tetraplegia: a multicenter study. Arch Phys Med Rehabil 2000;81:389-393.

43. Marino RJ, Ditunno JF, Jr., Donovan WH, Maynard F, Jr. Neurologic recovery after traumatic spinal cord injury: data from the Model Spinal Cord Injury Systems. Arch Phys Med Rehabil 1999;80:1391-1396.

44. Steeves JD, Kramer JK, Fawcett JW, et al. Extent of spontaneous motor recovery after traumatic cervical sensorimotor complete spinal cord injury. Spinal Cord 2011;49:257-265.

45. Wirth B, van Hedel HJ, Kometer B, Dietz V, Curt A. Changes in activity after a complete spinal cord injury as measured by the Spinal Cord Independence Measure II (SCIM II). Neurorehabil Neural Repair 2008;22:145-153.

46. Wallace D, Duncan PW, Lai SM. Comparison of the responsiveness of the Barthel Index and the motor component of the Functional Independence Measure in stroke: the impact of using different methods for measuring responsiveness. J Clin Epidemiol 2002;55:922-928. 
47. Studenski S, Hayes RP, Leibowitz RQ, et al. Clinical Global Impression of Change in Physical Frailty: development of a measure based on clinical judgment. Journal of the American Geriatrics Society 2004;52:1560-1566. 



\title{
Epicritic sensation in cervical spinal cord injury: diagnostic gains beyond testing light touch
}

\author{
Inge-Marie Velstra \\ Marc Bolliger \\ Michael Baumberger \\ Johan S. Rietman \\ Armin Curt
}

J Neurotrauma 2013;30(15):1342-1348

Reprinted with permission. 


\section{Abstract}

Applied as a bedside test of gross dorsal column function, the testing of light touch sensation is of high clinical value in the diagnosis of human spinal cord injury (SCI). However, the assessment of overall dorsal column deficit by testing only light touch may be limited, because the dorsal column pathway conveys several large diameter afferent modalities (e.g., sensation of touch, two-point discrimination and proprioception). Therefore, the objective of this study was to compare the epicritic sensation assessed by light touch (LT), Semmes Weinstein Monofilament (SWM) and electrical perception threshold (EPT) across cervical dermatomes (C3 to C8) in individuals with cervical $\mathrm{SCl}$. A multicenter cross sectional study was performed at 6 months after cervical SCI applying combined measures of LT, SWM and EPT, bilaterally over predefined key sensory points ( $\mathrm{C} 3$ to $\mathrm{C} 8$ ). A total of 300 left- and right-sided dermatomes were tested for each outcome measure in 25 participants. The percentage agreement between classifications according to LT and SWM/EPT testing for all dermatomes between C3 and C8 ranged from $95.5 \%$ to $36.2 \%$. The degree of agreement showed considerably variable kappa coefficients $\left(-0.1 \geq k_{w} \leq 0.7\right)$ for each dermatome between C3 and C8. The additional measurements of epicritic sensation by SWM and EPT increased sensitivity by detecting and quantifying differences in sensory thresholds above, at and below the LT level of injury. This is relevant for early clinical trials (phase I/II), where disclosing any biological activity of an intervention may be revealed by subtle sensory changes that might gain a clinical relevance. 


\section{Introduction}

Testing of sensory function is essential to sufficiently measure the degree of dysfunction and recovery after acute cervical spinal cord injury (C-SCI), which is paramount for setting reasonable goals in rehabilitation and for accurate stratification in a clinical trial. ${ }^{1,2}$ However difficulties arise in selecting outcome measures that can detect small changes to evaluate the success of rehabilitation and to test the efficacy of new interventions, for the different sensory modalities and in the interpretation of those test results.

Light touch (LT) and pinprick (PP) assessment examined according to the International Standards for Neurological Classification of Spinal Cord Injury (ISNCSCI) 3 is used routinely during neurological examination of sensibility in patients with a $\mathrm{SCl}$. However, the sensory assessment by the ISNCSCI might not be sufficiently sensitive to monitor safety and/or to detect subtle therapeutic benefits. ${ }^{4}$ Furthermore, it may not be able to elucidate potential mechanisms of recovery. ${ }^{5}$ Therefore, additional quantitative sensory assessments, such as the Electrical Perception Threshold (EPT) and the Semmes and Weinstein Monofilament (SWM), may be used in combination with LT and PP to improve the sensitivity to discrete sensory changes and robustness of sensibility examination in the clinic and research. ${ }^{6-11}$ LT, SWM and EPT assess the posterior column pathway for detecting thresholds for tactile cutaneous sensation and electrical cutaneous sensation. ${ }^{7,12-14}$

So far SWM remains the only internationally recognized handheld instrument specifically designed to control application force variables, and to meet sensitivity and repeatability requirements for an objective outcome measure of sensation. ${ }^{10,15-17}$ However, to the best of our knowledge, SWM has not been systematically applied in spinal cord disorders, ${ }^{18}$ while clinical experience in peripheral nerve damage (i.e. nerve repair surgery in upper extremities) has proven its validity and is well established in clinical use. ${ }^{17,19}$

The pocket version of SWM consists of 5 nylon monofilaments with variable stiffness that apply different amount of grams to quantify cutaneous sensation. The SWM sensory threshold is defined as the force of the lightest filament at which the patient reports sensation. In participants with chronic cervical SCI, the SWM has been reported to have high validity and excellent overall inter- and intra-tester reliability (ICC ranged between 0.84-0.95). ${ }^{8}$ However, the ICC between individual cervical dermatomes has not been reported. Recently it has been shown that the ICC is fair for the SWM in C4, T1, T8 and L4 dermatomes in healthy subjects. ${ }^{20}$ However ICC values can vary between healthy subjects and patients as a result of inter individual variations of sensory thresholds as well as differences in sensibility between dermatomes. The determination of the SWM sensory threshold has the advantage of being a simple and unobtrusive/discreet method. 
EPT testing has been introduced in the assessment of sensory function in spinal cord disorders and holds promise in providing sensitive readouts beyond the clinical scaling. . $^{72,21-23}$ The EPT is defined as the lowest ascending electrical stimulus intensity expressed in $\mathrm{mA}$ at which the patient reports sensation. ${ }^{6,24}$ The overall inter- and intratester reliabilities of EPT appear to be moderate to good in healthy participants ${ }^{20,21}$ and participants with incomplete $\mathrm{SCl} .{ }^{24}$ However, the intraclass correlation coefficient (ICC) varied considerably between individual dermatomes in healthy participants. ${ }^{23}$ Thus there are different normative values for each dermatome. The EPT can be applied to all sensory dermatomes and results can be interpreted quickly, but the method is more time-consuming than LT testing.

The LT assessment of sensation roughly grades the ability of detecting a light touch in the affected dermatome by "absent", "impaired" or "normal". Thus detection of subtle improvements in sensation or minor sensory impairments is rather impossible. The SWM and EPT contain a greater range of discernable response levels for detecting a tactile cutaneous sensation and electrical stimulation and therefore have the potential of being more sensitive. ${ }^{22,25}$ This encompasses a wide range of degrees of impairment and can include hypersensitivity as well as hyposensitivity. However, it is unknown, if EPT or SWM are more sensitive than clinical sensory examination (LT) for an individual cervical dermatome.

While SCI physicians and clinicians have gained great experience of LT testing in the clinical management of patients suffering from SCl the actual sensitivity of LT testing to assess dorsal column function in patients with $\mathrm{SCl}$ is less established. To our knowledge there is no study that compares epicritic sensation assessed by LT, SWM and EPT across cervical dermatomes ( $\mathrm{C} 3$ to $\mathrm{C} 8$ ) in participants at 6 months after cervical SCl. The following study hypothesized that the segmental assessment of epicritic sensation in human $\mathrm{SCl}$ can be improved by additional semi-quantitative sensory measures complementary to LT. The latter findings are required for the consideration if LT testing can be assumed sensitive enough in interventional studies.

\section{Methods}

Study design

A cross-sectional multi-center study in two specialized SCI rehabilitation centers. 


\section{Study population}

Participants were recruited between March 2010 and May 2011 from two Swiss SCI centers: the University Hospital Balgrist, Zurich and the Swiss Paraplegic Center, Nottwil. Inclusion criteria consisted of traumatic or non-traumatic cervical SCI with an ASIA Impairment Scale (AIS) grade A, B, C, or D. ${ }^{3}$ Exclusion criteria were individuals with any accompanying severe neurological (e.g., traumatic brain injury) or medical disorders and age less than 16 years. The participants were enrolled after having providing written informed consent. The local Ethics Committees of the two participating centers approved the study.

\section{Procedures}

The assessors were clinicians (physicians and occupational therapists) who had long lasting experience in working with individuals with $\mathrm{SCl}$. To ensure high-quality and reliable examinations, assessors were trained how to perform all applied outcome measures. A standardized protocol that outlined detailed instructions on performing the assessments was followed for each outcome measure. The recording techniques and materials were standardized across both centers. All participants were tested in a quiet room throughout the exams. For EPT and LT testing, participants were lying in a comfortable supine position, and for SWM testing participants were seated. First the testing procedure was explained to the participants. Before testing of cervical dermatomes, the stimuli of the different outcome measures were applied to a dermatome with normal sensation, i.e. the face, in order for the patient to recognize the sensation. Subsequently, the participants were asked to close both of their eyes, and the testing was started. All outcome measures were assessed in a random order at 6 months after SCI (defined as a time window between 150-186 days).

\section{Assessments}

The SWM and EPT outcome measures were applied bilaterally over predefined ASIA sensory key points in the dermatomes C3 to C8.

The clinical neurological examination of touch sensation was assessed by the ASIA $\mathrm{LT}$ testing according to the ISNCSCI protocol for the whole body. ${ }^{3}$ The PP assessment involves the anterior column pathway (i.e. spino-thalamic fiber tracts) and is therefore not included in the present study. Appreciation of LT sensation at each of the ASIA sensory key points was scored on an ordinal three-point scale as follows: $2=$ normal; $1=$ impaired and $0=$ absent. The LT level of lesion was defined as the last intact sensory level as indicated by normal LT testing. ${ }^{3}$ 
The tactile cutaneous sensation threshold was assessed by the pocket version of SWM $^{10}$ (North Coast Medical, Inc, Campbell, Canada) according to a strict and standardized assessment protocol. ${ }^{10}$ The sensory threshold of the SWM was defined as the force of the lightest filament at which the individual reports sensation. An ascending method of threshold testing was used, starting with the smallest diameter monofilament (lightest filament, lowest force, most difficult to detect) and continuing in order of increasing diameter if the patient did not respond to the previous filament. Only ASIA sensory key point locations, which did not respond to the previous filament, were tested with the next filament. The exam was continued until the patient recognized a force/touch in all test locations or until it was established that the patient did not feel even the heaviest filament. Two of three applications of the lightest filament had to be felt, to obtain a positive result. All the other, heavier filaments were applied only once according to the manufacturer's instructions. ${ }^{10}$ In the study of Voerman et al., ${ }^{13}$ filament marking 3.61 represents the normal value for sensory thresholds in all cervical dermatomes. In the present study the qualification of normal values was based on the mean threshold and the $95 \% \mathrm{Cl}$ according to Voerman et al. ${ }^{13}$ The log of grams of force were represented by numeric values ranging from 0 to 4 as described in the instructions of the SWM mini-kit: $4=$ filament $3.61 ; 3=$ filament 4.31; 2 = filament 4.56; 1 = filament 6.65 and $0=$ no response..$^{10,13,16}$ In our study, a SWM value of 1,2 or 3 points was defined as impaired, 4 points were defined as normal and 0 points was defined as absent.

The EPT ${ }^{6,22}$ was assessed according to previous studies, ${ }^{6,22}$ using a modified mobile Compex 2 stimulator (Compex Medical SA, Switzerland), which delivered a square shaped stimulus of $0.5 \mathrm{~ms}$ duration at $3 \mathrm{~Hz}$. The perceptual threshold was defined as the lowest ascending stimulus intensity $(\mathrm{mA})$ at which the patient reported sensation. The maximal stimulator-output and the smallest increment were adjusted to $26.9 \mathrm{~mA}$ and $0.21 \mathrm{~mA}$, respectively. The skin was thoroughly cleaned with alcohol wipes, and disposable, selfadhesive ECG-electrodes (cathode) with a diameter of 18mm (3M Red DotTM - type 2248) were applied over the ASIA sensory key points. A large $(50 \times 90 \mathrm{~mm})$ inactive electrode (anode; Synapse Electrodes, Ambu, Denmark) was attached to the forearm of the testing side. For every dermatome tested, the stimulus intensity was manually increased and decreased with changes applied exactly once per second, until the patient first reported the sensation (ascending) under the cathode. This was repeated three times and the lowest EPT (expressed in $\mathrm{mA}$ ) of the three measurements was included in the analysis. Van Hedel et al., ${ }^{23}$ have established the normal values for the electrical perception threshold for each 
cervical dermatome. In our study the qualification of normal values was based on the mean value of the upper limits of the $95 \% \mathrm{Cl}$ from the two measurements which reflects normality according to van Hedel et al. ${ }^{23}$ In the present study, an impaired EPT value was defined as any value greater than the normative EPT value. A normal EPT value was defined as any value equal or smaller than the normative EPT value, and the EPT value was considered absent, if the maximum current intensity (threshold at $8.4 \mathrm{~mA}$ ) was not perceived. A stimulation above $8.4 \mathrm{~mA}$ was avoided as at this level of intensity also additional pathways (i.e. nociceptive A-delta or C-fibers) than dorsal column fibers (i.e. A-beta fibers) might become effectively stimulated and could falsify the perceived sensation by the subjects. ${ }^{12}$

\section{Statistical analysis}

Descriptive statistics were used to determine the frequency, median and range of the study participants' characteristics including cause of injury, AIS grade, AIS sensory LT level, sex and age. For comparison with LT scores the study participants' SWM and EPT data were classified as normal, impaired or absent and scored with 2, 1 or 0 , respectively. All comparisons were made for the right and left side combined, because there was no statistically significant difference between the right and left side. Dermatomes were classified as having abolished, impaired or normal sensation based on LT, SWM and EPT testing. The frequency and percentage of classification agreement between LTSWM and LT-EPT for all C3 to C8 dermatomes was determined. Finally the degree of agreement between the three measures, weighted (Fleiss-Cohen) kappa coefficients and confidence intervals were calculated for each dermatome between C3 and C8 as well as for all dermatomes between C3 and C8. Agreement was assessed using the standards as established by Altman: 0.00 , poor; $0.01-0.20$, slight; $0.21-0.40$, fair; $0.41-0.60$, moderate; $0.61-0.80$, substantial; and $0.81-1.00$, almost perfect. ${ }^{26}$

All data were analysed using SPSS version 18.0 for Windows and $\mathrm{R}$ version 2.15.1 for Windows.

\section{Results}

\section{Participants}

Table 4.1 presents the characteristics of the 25 cervical SCl participants with a high percentage of incomplete SCl. SCl was scored as complete (AIS A) in five individuals and incomplete in the remaining 20 individuals (AIS B, C and D). In five individuals the 
Table 4.1 Demographic and clinical characteristics of participants $(n=25)$

\begin{tabular}{lc}
\hline Characteristics & All participants \\
\hline Cause of SCI & \\
Traumatic & $23(92 \%)$ \\
Non-traumatic & $2(8 \%)$ \\
Site & \\
Uniklinik Balgrist Zurich (CH) & $3(12 \%)$ \\
Swiss Paraplegic Centre Nottwil (CH) & $22(88 \%)$ \\
Sex & \\
Females & $5(20 \%)$ \\
Males & $20(80 \%)$ \\
Age (years) & \\
Median & 56 \\
Min-max & $20-84$ \\
AIS & \\
A & $5(20 \%)$ \\
B & $3(12 \%)$ \\
C & $1(4 \%)$ \\
D & $16(64 \%)$ \\
Sensory light touch level & \\
C2 & $1(4 \%)$ \\
C3 & $3(12 \%)$ \\
C4 & $7(28 \%)$ \\
C5 & $4(16 \%)$ \\
C6 & $3(12 \%)$ \\
C7 8 & $1(4 \%)$ \\
Below C8 & $1(4 \%)$ \\
No detectable light touch level & $2(8 \%)$ \\
\hline A & $3(12 \%)$ \\
\hline &
\end{tabular}

Abbreviations: $\mathrm{SCl}$, spinal cord injury; $\mathrm{n}$, sample size;

AIS, American Spinal Injury Association Impairment Scale.

cervical dermatomes did not reveal any touch sensation disturbance. A total of 300 leftand right-sided dermatomes were tested for each outcome measure between C3 and C8 in all 25 participants.

\section{Distribution of findings in LT, SWM and EPT}

Classification of dermatomes according to LT, SWM and EPT testing are shown in Figure 4.1. The greatest number of dermatomes was classified as intact when using LT testing (62.7\%), while applying SWM (44.3\%) and EPT (29.3\%) revealed fewer intact dermatomes. Accordingly, the number of dermatomes classified as impaired increased from LT (30\%) 


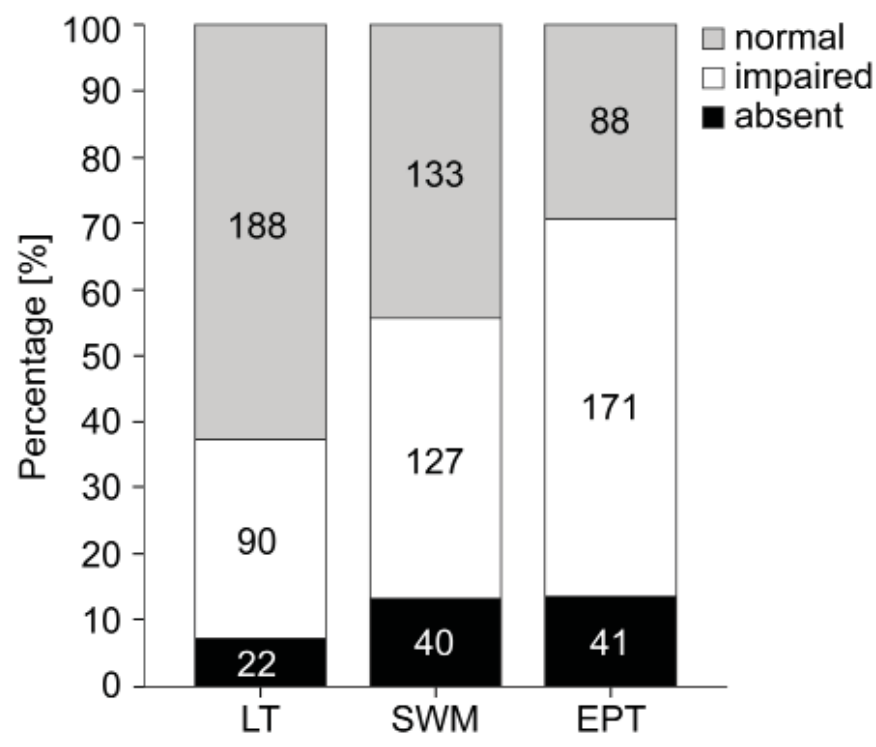

Figure 4.1 Numbers and percentage of dermatomes in absent, impaired and normal sensation for light touch (LT), Semmes and Weinstein Monofilament (SWM), and electrical perception threshold (EPT). A total of 300 dermatomes for each outcome measure between cervical 3 and cervical 8 were tested.

to SWM testing (42.3\%) and being greatest for EPT testing (57\%) across dermatomes. The number of dermatomes classified as absent were rather similar for SWM and EPT testing $(30.3 \%$ and $30.7 \%)$ and lower for LT $(7.3 \%)$.

\section{Agreement of LT and SWM classification}

The agreement of classification between LT and SWM for all dermatomes between C3 and C8 is reported in Table 4.2. The overall agreement of classifications comparing LT and SWM within same categories was for absent sensation 95.5\% (21/22), for impaired sensation 47.8\% (43/90) and for normal sensation 54.3\% (102/188). In dermatomes with absent light touch sensation (LT 0), 4.5\% reported some SWM sensation. However in dermatomes with preserved LT, either impaired (LT 1) or normal LT (LT 2), a high degree of discordance was observed compared to SWM. In dermatomes with normal LT, the SWM testing classified $44.1 \%$ (83/188) of dermatomes as being impaired. All 83 dermatomes classified by impaired SWM were at and above the LT level of injury. In dermatomes classified with impaired LT scores, SWM testing revealed normal or absent sensation in $34.4 \%$ (31/90) and 17.8\% (16/90), respectively. All 31 dermatomes classified by normal SWM were below the LT level of injury. 


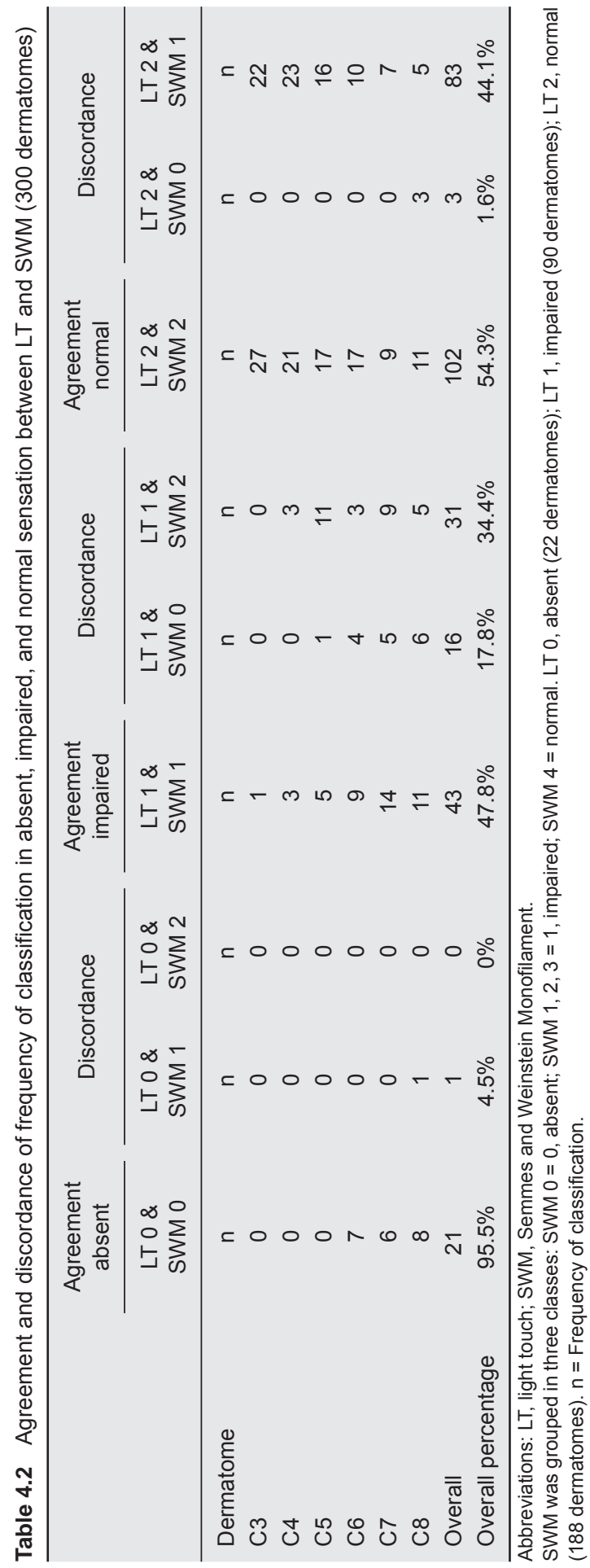


Agreement of LT and EPT classification

The agreement of classifications between LT and EPT for all dermatomes between C3 and C8 are reported in Table 4.3. Overall the agreement between classifications examined by LT and EPT testing was $90.9 \%$ (20/22) for absent of sensation, $52 / 90$ (57.8\%) for impaired sensation and 68/188 (36.2\%) for normal sensation. A high discordance of classifications for impaired and normal sensation between LT and EPT were recorded in the remaining dermatomes. In dermatomes with normal LT, the EPT testing classified 62.2\% (117/188) of dermatomes as being impaired. All 117 classified by impaired EPT were at and above the LT level of injury. Furthermore, in dermatomes classified with impaired LT scores, EPT testing revealed normal sensation in 22.2\% (20/90) and an absent sensation in 20\% (18/90). All 20 dermatomes classified by normal EPT were below the LT level of injury.

\section{Degree of agreement between LT-SWM and LT-EPT}

Table 4.4 shows the level of classification agreement between LT-SWM and LT-EPT for all dermatomes between C3 and C8. The overall weighted kappa coefficient between LT and SWM was moderate (0.5) and fair (0.4) for LT-EPT. However, when evaluated for individual dermatomes the agreement varied considerably. In dermatomes C3, C4 and C5 the weighted kappa coefficients $(\leq 0.2)$ revealed that the level of classification agreement between LT and SWM, as well as between LT and EPT, occurred rather by chance. However in dermatomes C6, C7 and C8 the weighted kappa coefficients was found moderate to substantial (weighted kappa range $=0.5$ to 0.7 ) between the different testing methods.

\section{Discussion}

The study reveals for the first time a comprehensive comparison of epicritic sensation as assessed by LT, SWM and EPT across cervical dermatomes (C3 to C8) in individuals with $\mathrm{SCl}$. The sensory testing focused on the assessment of sensory integrity of distinct predefined dermatomes in patients suffering from cervical SCI. Improving the assessment of epicritic sensation will be important in early clinical trials (phase I/II), where segmental and subtle changes in sensory function might provide important readouts about beneficial as well as detrimental (i.e., descending and ascending levels of lesion, respectively) effects of novel interventions. The presented study provides evidence that the segmental assessment of epicritic sensation can be improved by SWM and EPT. 

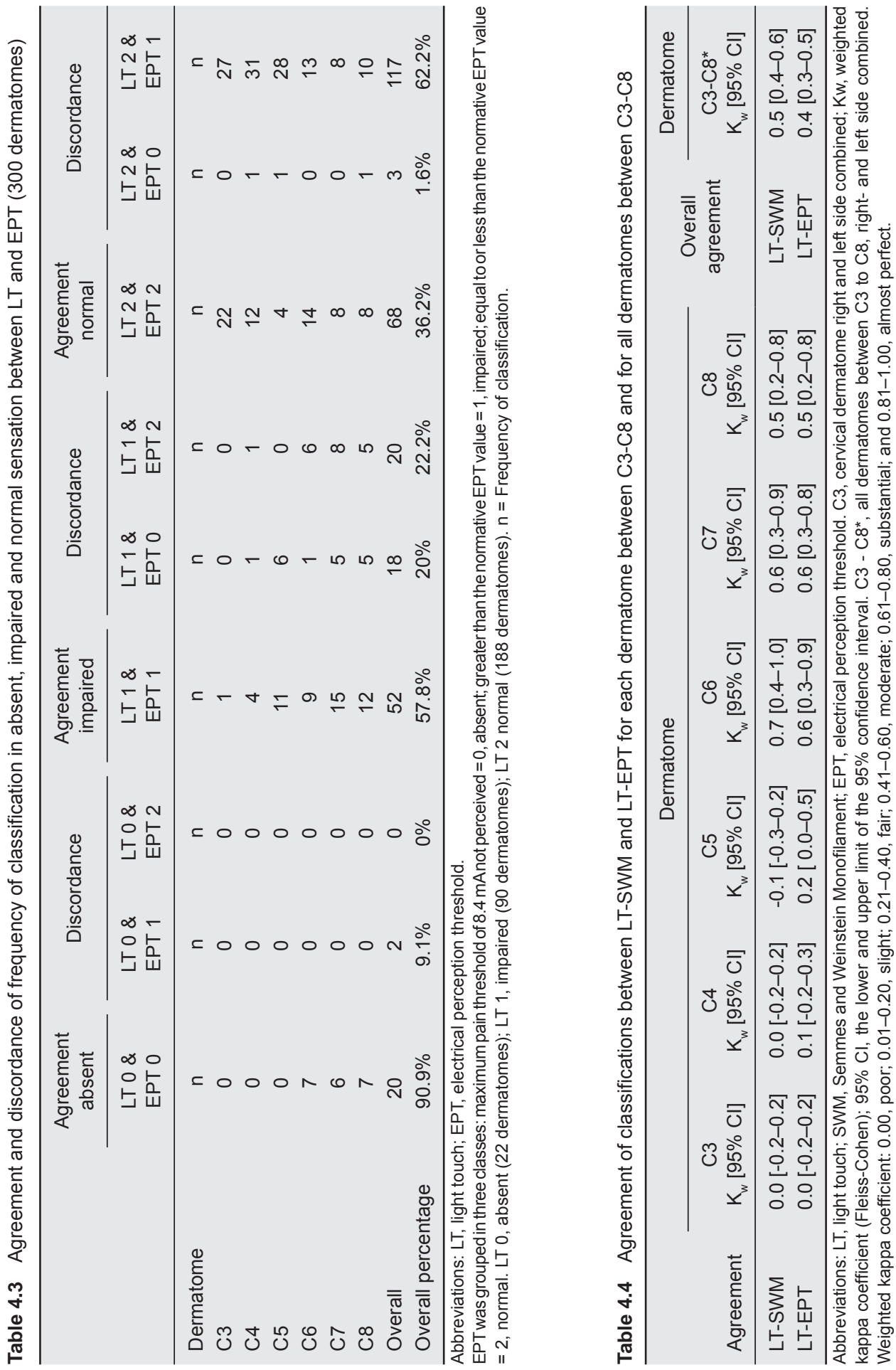
The challenge of sensory testing

Clinical testing of sensory function is commonly challenged by limitations of test reliability, which is true to some extent for almost all sensory qualities (like epicritic sensation and prothopatic sensation). ${ }^{7,9,27,28}$ This becomes even more demanding when assessing different levels of sensory impairment where the subjective rating of patients is not able to define incremental levels of impairment but becomes rather limited to a simplified categorical (ordinal scale, e.g. normal, impaired and abolished sensation) gross scoring. Therefore, the ability to understand minor changes during recovery (improvements or deterioration) is very challenging and clinical testing of one specific sensory quality (like LT) within a complex domain of sensory function (like epicritic sensation conveyed by dorsal column pathways) will be likely of limited sensitivity. One approach to overcome these challenges is to introduce measures with a more defined scaling of sensation (like SWM testing by applying different sensory modalities) or to combine complementary sensory measures that are considered to reflect to some extent the integrity within similar fiber tracts. The latter approach would require modalities that are not redundant, but are able to reveal subtle differences regarding the integrity of function within an entire sensory system. In this context the applied measures should also represent the same anatomical areas (i.e. distinct dermatomes) which can be well achieved by using LT, SWM and EPT testing.

\section{Disparity and sensitivity}

The value of combining sensory testing of EPT and SWM complementary LT testing resides in the intention that they provide different insights in the integrity or impairment of epicritic sensation. Accordingly they should not be just redundant but reveal changes that cannot be disclosed by LT testing. Indeed a mismatch of segmental epicritic sensation was observed as SWM was classified in $44.1 \%$ dermatomes and EPT classified in $62.2 \%$ dermatomes as impaired whereas LT revealed normal response. Interestingly those findings were all at or above the LT level according to ISNCSCI and are for the EPT findings in accordance

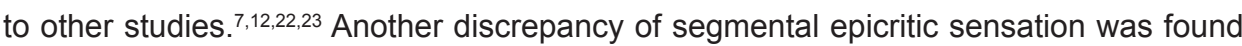
below the level of injury according to ISCNSCI. LT revealed impaired response whereas SWM classified $34.4 \%$ dermatomes and EPT classified $22.2 \%$ dermatomes as normal. These SWM findings are in agreement with the results of Kalsi-Ryan et al. ${ }^{8}$ In their study, they used SWM in individuals with chronic cervical SCI and observed greater sensitivity when using SWM, due to increased response levels, compared to the values reported when using ISNCSCI light touch. The EPT findings are in line with the results of Kramer 
et al. ${ }^{21}$ who observed that individuals with cervical SCI have persisting EPT values below the level of lesion. ${ }^{22}$ The present results suggest that SWM and EPT might be sensitive to small sensory impairments and/or preserved innervation in sensory function above, at and below the LT level, which are less detectable by LT testing. This degree of sensitivity could be required to assess differences in sensory recovery, especially when improvements might be limited to one or two dermatomes adjacent to the LT level. Obviously the value of additional sensory testing is most relevant in dermatomes that are clinically considered to be normal or impaired. In dermatomes with abolished sensation SWM and EPT do not provide additional information to LT testing (overall agreement for abolished C3 to C8 dermatomes betweenLT and SWM / EPT testing was about $95.5 \%$ and $90.9 \%$, respectively).

Statistical analysis $\left(k_{w} \leq 0.5\right)$ confirmed that there is only a limited congruency between the three different assessments addressing epicritic sensation. Interestingly these findings were not uniform across all dermatomes and revealed a higher percentage of congruency (moderate to substantial agreement) specifically in the C6, C7 and C8 dermatomes where a higher percentage of absent sensation was found with all three testing methods. Furthermore, in the C3, C4 and C5 dermatomes a higher percentage of impaired and normal sensation was reported which revealed a poor agreement between the three different assessments. These findings emphasize, as has been shown by the different thresholds for SWM and EPT across these dermatomes, that the clinical assessment using LT is of limited sensitivity to disclose segmental differences in sensory function. Differences in findings across cervical (and thoracic) dermatomes are not specific for epicritic sensation but have been also shown for the assessment of spino-thalamic function (i.e. using laser evoked potentials or contact heat evoked potentials) that reveal marked difference between dermatomes (again these differences between dermatomes are also not adequately reflected by the clinical testing of pin prick sensation). ${ }^{29,30}$

\section{Improving readouts of sensory plasticity}

The aims of increasing the sensitivity of testing epicritic sensation after SCl are two-fold: 1) to identify changes within dermatomes, i.e. high resolution of segmental changes, and 2) to provide insight into specific pathways that for the epicritic sensation are characterized by their high level of myelination. Therefore, applying such measures in an interventional study can address if segmental changes occur that are beyond spontaneous or regular findings (both beneficial and detrimental). In addition such measures might be useful if interventions are considered to improve the myelination (i.e. concept of re-myelination) 
of damaged spinal fibers, where the recovery of A beta fibers depend on high level of myelination and might reveal superior recovery than less or un-myelinated sensory fibers (like C fibers). ${ }^{31,32}$ Therefore, in clinical trials an improved resolution of sensory function by combined LT and SWM/EPT testing could be meaningful in revealing subtle changes that for a proof of mechanism might be critical to enter a next phase where these effects can be amplified by adjusting the intervention.

\section{Conclusion}

There is limited agreement of sensory testing specifically in incompletely affected dermatomes between testing of LT, SWM and EPT. This difference is likely attributable to the measurement limitations of each testing and that they individually respond to the differently affected sensory modalities within the epicritic sensation. ${ }^{28}$ The results show that SWM and EPT testing can add complementary resolution to LT testing at 6 months after cervical $\mathrm{SCI}$ by detecting and quantifying differences in sensory thresholds above, at and below the LT level of injury. The ability of combined sensory testing to gain insights beyond LT warrants consideration in the protocol design of interventional studies where the sensitivity to indicate even subtle differences is of value both in the stratification of patients and the potential (not shown here) to reveal an improved responsiveness in sensory testing.

\section{Acknowledgements}

The authors thank the individuals with SCI participating in this study and the contribution of the staff at the Balgrist University Hospital Zurich and Swiss Paraplegic Centre Nottwil. We thank Dr. Jörg Krebs, Clinical Trial Unit, Swiss Paraplegic Centre Nottwil and Dr. John Kramer, Balgrist University Hospital Zurich for critical review and editorial assistance of the manuscript.

\section{References}

1. Steeves JD, Lammertse D, Curt A, et al. Guidelines for the conduct of clinical trials for spinal cord injury (SCI) as developed by the ICCP panel: clinical trial outcome measures. Spinal Cord 2007; 45:206-221.

2. Curt A, Schwab ME, Dietz V. Providing the clinical basis for new interventional therapies: refined diagnosis and assessment of recovery after spinal cord injury. Spinal Cord 2004;42:1-6.

3. Kirshblum SC, Waring W, Biering-Sorensen F, et al. Reference for the 2011 revision of the International Standards for Neurological Classification of Spinal Cord Injury. J Spinal Cord Med 2011;34:547-554. 
4. Steeves JD, Fawcett JW, Tuszynski MH, et al. Experimental treatments for spinal cord injury: what you should know if you are considering participation in a clinical trial. A guide for people with spinal cord injury, their families, friends \& caregivers, 2007.

5. Fawcett JW, Curt A, Steeves JD, et al. Guidelines for the conduct of clinical trials for spinal cord injury as developed by the ICCP panel: spontaneous recovery after spinal cord injury and statistical power needed for therapeutic clinical trials. Spinal Cord 2007;45:190-205.

6. Davey NJ, Nowicky AV, Zaman R. Somatopy of perceptual threshold to cutaneous electrical stimulation in man. Exp Physiol 2001;86:127-130.

7. Hayes KC, Wolfe DL, Hsieh JT, Potter PJ, Krassioukov A, Durham CE. Clinical and electrophysiologic correlates of quantitative sensory testing in patients with incomplete spinal cord injury. Arch Phys Med Rehabil 2002;83:1612-1619.

8. Kalsi-Ryan S, Beaton D, Curt A, et al. The Graded Redefined Assessment of Strength Sensibility and Prehension: reliability and validity. J Neurotrauma 2012;29:905-914.

9. Krassioukov A, Wolfe DL, Hsieh JT, Hayes KC, Durham CE. Quantitative sensory testing in patients with incomplete spinal cord injury. Arch Phys Med Rehabil 1999;80:1258-1263.

10. Bell-Krotoski JA. Sensibility testing: current concepts. In: Hunter JM, Mackin EJ, Callahan AD, eds. Rehabilitation of the hand: surgery and therapy. St Louis CV Mosby, 1995: 129-152.

11. Savic G, Bergstrom EM, Frankel HL, Jamous MA, Jones PW. Inter-rater reliability of motor and sensory examinations performed according to American Spinal Injury Association standards. Spinal Cord 2007;45:444-451.

12. Kramer JL, Moss AJ, Taylor P, Curt A. Assessment of posterior spinal cord function with electrical perception threshold in spinal cord injury. J Neurotrauma 2008;25:1019-1026.

13. Voerman VF, van Egmond J, Crul BJ. Normal values for sensory thresholds in the cervical dermatomes: a critical note on the use of Semmes-Weinstein monofilaments. Am J Phys Med Rehabil 1999;78:24-29.

14. Beric A, Dimitrijevic MR, Lindblom U. Cortical evoked potentials and somatosensory perception in chronic spinal cord injury patients. Journal of the neurological sciences 1987;80:333-342.

15. Bell-Krotoski JA, Buford WL, Jr. The force/time relationship of clinically used sensory testing instruments. J Hand Ther 1997;10:297-309.

16. Bell-Krotoski JA, Fess EE, Figarola JH, Hiltz D. Threshold detection and Semmes-Weinstein monofilaments. J Hand Ther 1995;8:155-162.

17. Rosen B, Dahlin LB, Lundborg G. Assessment of functional outcome after nerve repair in a longitudinal cohort. Scandinavian journal of plastic and reconstructive surgery and hand surgery / Nordisk plastikkirurgisk forening [and] Nordisk klubb for handkirurgi 2000;34:71-78.

18. Velstra IM, Ballert CS, Cieza A. A systematic literature review of outcome measures for upper extremity function using the international classification of functioning, disability, and health as reference. PM R 2011;3:846-860.

19. Jerosch-Herold C. Assessment of sensibility after nerve injury and repair: a systematic review of evidence for validity, reliability and responsiveness of tests. J Hand Surg Br 2005;30:252264.

20. Ellaway PH, Catley M. Reliability of the electrical perceptual threshold and Semmes-Weinstein monofilament tests of cutaneous sensibility. Spinal Cord 2013;51:120-125. 
21. Kramer JK, Taylor P, Steeves JD, Curt A. Dermatomal somatosensory evoked potentials and electrical perception thresholds during recovery from cervical spinal cord injury. Neurorehabil Neural Repair 2010;24:309-317.

22. Savic G, Bergstrom EM, Frankel HL, Jamous MA, Ellaway PH, Davey NJ. Perceptual threshold to cutaneous electrical stimulation in patients with spinal cord injury. Spinal Cord 2006;44:560566.

23. van Hedel HJ, Kumru H, Rohrich F, Galen S. Changes in Electrical Perception Threshold Within the First 6 Months After Traumatic Spinal Cord Injury: A Multicenter Responsiveness Study. Neurorehabil Neural Repair 2011.

24. King NK, Savic G, Frankel H, Jamous A, Ellaway PH. Reliability of cutaneous electrical perceptual threshold in the assessment of sensory perception in patients with spinal cord injury. J Neurotrauma 2009;26:1061-1068.

25. Kalsi-Ryan S, Curt A, Verrier MC, Fehlings MG. Development of the Graded Redefined Assessment of Strength, Sensibility and Prehension (GRASSP): reviewing measurement specific to the upper limb in tetraplegia. Journal of neurosurgery Spine 2012;17:65-76.

26. Altman DG. Practical statistics für medical research. Boca Raton: CRC, 1991.

27. Cohen ME, Sheehan TP, Herbison GJ. Content validity and reliability of the International Standards for Neurological Classification of Spinal Cord Injury. Top Spinal Cord Inj Rehabil 1996:15-31.

28. Jonsson M, Tollback A, Gonzales H, Borg J. Inter-rater reliability of the 1992 international standards for neurological and functional classification of incomplete spinal cord injury. Spinal Cord 2000;38:675-679.

29. Haefeli J, Curt A. Refined sensory measures of neural repair in human spinal cord injury: bridging preclinical findings to clinical value. Cell and tissue research 2012;349:397-404.

30. Kramer JL, Taylor P, Haefeli J, et al. Test-retest reliability of contact heat-evoked potentials from cervical dermatomes. Journal of clinical neurophysiology : official publication of the American Electroencephalographic Society 2012;29:70-75.

31. Powers BE, Lasiene J, Plemel JR, et al. Axonal thinning and extensive remyelination without chronic demyelination in spinal injured rats. The Journal of neuroscience : the official journal of the Society for Neuroscience 2012;32:5120-5125.

32. Mekhail M, Almazan G, Tabrizian M. Oligodendrocyte-protection and remyelination post-spinal cord injuries: a review. Progress in neurobiology 2012;96:322-339. 



\title{
Prediction and stratification of upper limb function and self-care in acute cervical spinal cord injury (SCl) with the Graded Redefined Assessment of Strength, Sensibility and Prehension \\ (GRASSP)
}

\author{
Inge-Marie Velstra \\ Marc Bolliger \\ Lorenzo Tanadini \\ Michael Baumberger \\ Rainer Abel \\ Johan S. Rietman \\ Armin Curt
}

Neurorehabil Neural Repair 2014;28(7):632-642 Copyright $\odot$ (2014) by The American Society of Neurorehabilitation. Reprinted by permission of SAGE Publications, Inc. 


\section{Abstract}

Background: There is inherent heterogeneity within individuals suffering from cervical spinal cord injury (SCI), and early prediction of upper limb function and self-care is challenging. As a result, considerable uncertainty exists regarding the prediction of functional outcome following cervical $\mathrm{SCl}$ within 1 year of injury.

Objective: To evaluate the value of Graded Redefined Assessment of Strength, Sensibility, and Prehension (GRASSP) in predicting upper limb function and self-care outcomes in individuals with cervical SCI.

Method: A prospective longitudinal multicenter study was performed. Data from the GRASSP, the Spinal Cord Independence Measure (SCIM III), and the American Spinal Injury Association (ASIA) Impairment Scale were recorded at 1, 6, and 12 months after cervical SCI. For prediction of functional outcome at 6 and 12 months, a logistic regression model, receiver operating characteristics (ROC), and unbiased recursive partitioning conditional inference tree (URP-CTREE) were used with 8 different predictor variables. Results: Logistic regression analysis, ROC analysis, and URP-CTREE all revealed that the strength subtest within GRASSP is the strongest predictor for upper limb function and self-care outcomes. URP-CTREE provides useful information on the distribution of different outcomes in acute cervical $\mathrm{SCl}$ and can be used to predict cohorts with homogeneous outcomes.

Conclusion: The GRASSP at 1 month can accurately predict upper limb function and self-care outcomes even in a heterogeneous group of individuals across a wide spectrum of neurological recovery. The application of URP-CTREE can reveal the distribution of outcome categories and, based on this, inform trial protocols with respect to outcomes analysis and patient stratification. 


\section{Introduction}

Individuals with cervical spinal cord injury (SCI) suffer from a broad spectrum of upper limb impairments. They may experience loss of strength, sensation and movements, as well as limited ability to perform activities of daily living (ADLs). This ultimately leads to decreased independence and restricted participation as well as quality of life. Indeed, individuals with cervical SCI report a strong desire to regain arm and hand function and that such a gain would improve their independence and quality of life..$^{1,2}$ Therefore, reliable prediction of future upper limb function and self-care at an early stage after cervical $\mathrm{SCl}$ has become increasingly important for several reasons; clinically it would help in treatment planning and goal setting, in a research context it would permit evaluation of novel interventions and patient stratification ${ }^{3-6}$ and, from a socioeconomic perspective, would be of benefit in predicting the likely degree of capacity for independent living and required level of caregiver support. After a lesion of the cervical spinal cord, arm and hand function outcomes vary significantly and are not only dependent on the level and completeness of the lesion but also on the degree of recovery, motivation and occupational performance of the individual. This inherent heterogeneity within individuals following cervical $\mathrm{SCl}^{7,8}$ renders early prediction of upper limb function and self-care challenging. ${ }^{9}$

Although several outcome measures of upper limb function are available ${ }^{10}$ only a few have been specifically developed for $\mathrm{SCl}$ and psychometric testing of these measures has revealed deficits. ${ }^{10,11}$ The predictive validity of quantitative measures has not yet been established, ${ }^{12}$ while the aforementioned cohort heterogeneity in cervical SCI makes identifying appropriate outcome measures difficult. ${ }^{7,13}$ To overcome this limitation, the Graded and Redefined Assessment of Strength, Sensibility and Prehension (GRASSP) was developed as a quantitative outcome measure specific to upper limb function in cervical SCI. Most importantly, GRASSP covers different aspects of upper limb function in order to evaluate changes within the motor and sensory systems and how changes in the level of impairment contribute to complex upper limb functional tasks. ${ }^{14}$ In individuals with chronic cervical SCI (i.e. more than 6 months post-injury), the GRASSP has shown high validity and excellent overall inter- and intra-rater reliability, ${ }^{15}$ while analysis of responsiveness is still pending.

Little has been published on prediction of functional outcome in general following $\mathrm{SCl}^{16-19}$ and, in particular, data on prediction and stratification of upper limb function and self-care after incomplete cervical $\mathrm{SCl}$ is lacking. ${ }^{8}$ The aim of the study, therefore, was to evaluate the predictive value of GRASSP for upper limb function and self-care outcome at 6 and 12 months post-injury in individuals with acute cervical SCI. 


\section{Methods}

\section{Study design}

Prospective longitudinal multicenter study.

\section{Study population}

Participants were recruited from five European SCI centers specialized in the rehabilitation of individuals with spinal cord injury (SCI). Participants were recruited between January 2009 and May 2011. Inclusion criteria consisted of traumatic or non-traumatic, acute (16-40 days after injury) tetraplegia with an ASIA Impairment Scale (AIS) grade of A, B, C, or D. ${ }^{20}$ Patients were included if their injury was between $C 3$ and T1 in the case of ASIAA patients and C1-T1 in those with incomplete injuries. Excluded were those individuals with any accompanying severe neurological (e.g., traumatic brain injury) or medical disorders or aged less than 16 years. Participants were recruited after providing written informed consent and the study was approved by the relevant local ethics committees.

\section{Procedures}

Assessors (physicians and occupational therapists) with at least one year's experience in working with individuals with SCl performed the measurements. To ensure high-quality examinations and to reduce inter-observer variability, ${ }^{21}$ assessors received training in how to perform all outcome measure assessments. A unified protocol, outlining in detail how the assessments should be performed, was followed for each outcome measure, with recording techniques and materials standardized across all centers. A quiet room, free of distractions was used for all assessments. For ASIA testing, participants lay in a comfortable supine position while for GRASSP testing they were seated. The SCIM III was scored by trained and experienced physical therapists, nurses and occupational therapists. Assessments were performed at 1 month (range 16-40 days), 6 months (range 150-186 days) and 12 months (range 300-400 days) after cervical SCI.

The AIS classifications were calculated by a computer algorithm, ${ }^{22}$ in accordance with definitions in the International Standards for Neurological Classification of Spinal Cord Injury. ${ }^{20}$

\section{Predictor variables}

The GRASSP is an upper limb outcome measure for individuals with tetraplegia which includes manual muscle testing (MMT), Semmes and Weinstein monofilament (SWM) 
testing, adoption of three prehensile positions (qualitative grasping [Q|G]) and performance of six task-oriented prehension skills (quantitative grasping [QtG]). The subtests within GRASSP, assessed between day 16 and 40 after cervical SCI, were selected as baseline predictor variables:

Manual Muscle Testing (MMT). Strength was assessed for both arms using the $\mathrm{MMT}^{23}$ in 10 muscles of the upper limb (three in the arm, seven in the hand). Each item (muscle) was given a score varying from 0 (response absent) to 5 (normal power). The sum of the MMT subtest score for both sites therefore ranges from 0 to 100 points and the sum of the distal (hand) muscle group of the MMT subtest score for both sites ranges from 0 to 70 points.

Semmes and Weinstein Monofilament (SWM). The tactile cutaneous sensation threshold was assessed with the pocket version of SWM ${ }^{24}$ (North Coast Medical, Inc, Campbell, CA) with four probes on three dorsal and palmar sensory test locations in each hand as described in the instructions of the SWM mini-kit ${ }^{24}$ and the GRASSP manual. The pressure applied was recorded on an ordinal scale corresponding to numeric values varying from 0 (absent) to 4 (normal). The sum of the dorsal or palmar sensation subtest score for both sites therefore ranges from 0 to 24 points.

Qualitative grasping (Q/G). The ability of the participant to perform a cylindrical grasp, lateral key pinch and tip-to-tip pinch was assessed for both hands. Each grasp was given a score varying from 0 (no voluntary control of wrist and digits when grasping) to 4 (normal voluntary control of wrist and digits when generating the grasp). The sum of the grasping quality subtest score for both upper limbs thus ranges from 0 to 24 points.

Quantitative grasping (QtG). The ability of the participant to perform six prehension tasks for each arm separately (like grasping or moving a coin) was assessed in a standardized way. The tasks were scored between 0 and 5 according to the grasp used. One minute and 15 seconds were allowed for the completion of each task and, if the individual was unable to perform the task within this time period, the individual was asked to move on to the next task. ${ }^{25}$ The maximum possible sum of the quantitative subtest score was 60 for both sites.

The SCIM III assesses independence in fundamental daily activities and is useful for measuring the status of, or improvement in, everyday functions relevant to individuals with SCI. ${ }^{26}$ The SCIM III has been shown to perform well under psychometric testing ${ }^{27-29}$ and the self-care subcategory (SCIM-SS) is particularly notable for its high inter-rater reliability and internal consistency. ${ }^{27}$ The SCIM III consists of three subcategories: (1) Self-care 
(SCIM-SS), (2) respiration and sphincter management, and (3) mobility. In our study, the SCIM III-SS was selected as a predictor variable. The sum of the SCIM-SS ranges from 0 to 20 points.

Clinical neurological examination was performed according to the ISNCSCI protocol. ${ }^{20}$ Injury characteristics were classified according to the neurological level of injury (NLI) and the overall AIS grade. The Upper Extremity Motor Score (UEMS) of ASIA was selected as predictor variable. Strength in five key muscle groups of the upper limb in both arms (two muscles in the arm, three in the hand) were scored between 0 (absent response) and 5 (normal power). The sum value of this score ranges from 0 and 100 for both sides.

\section{Outcome measures}

For the purpose of this paper, upper limb function is defined as the capacity to use the upper limb for skilled actions, such as reaching, grasping, and manipulation of objects used in daily life. The GRASSP subtest QtG is therefore taken as reflecting upper limb function. QtG and the SCIM-SS (for details, see above) were used as anchor outcome measures of upper limb function and self-care at 6 and 12 months after cervical SCI.

\section{Statistical analysis}

Descriptive statistics were used to determine the frequency, median and range of the study participants' characteristics including AIS grade, lesion level and lateralization, sex and age.

We dichotomized the two outcomes for the logistic regression analysis to assign patients into a failure or success group. For QtG, individuals were allocated to the failure group (0-36 points) if they met any of the following three conditions: i) not able to perform the task at all, ii) not able to complete the task, and iii) able to complete the task only by using an alternative (i.e. compensatory) grasp (not able to perform standard grasps). All individuals who were able to complete the task using the standard grasp were allocated to the success group (37-60 points), irrespective of any difficulties while performing the task. To distinguish between individuals who were dependent or independent with respect to self-care with or without devices, a cut-off SCIM-SS score of 12 was applied, with scores of 0-12 points defined as dependent (failure), and scores of 13-20 as independent (success), irrespective of supplementary device usage.

Binary logistic regression was performed on the dichotomized outcomes QtG and self-care with the goal to predict upper limb function and self-care at 6 and 12 months using predictors gathered between day 16 and 40 after cervical SCI. The number of 
predictors was minimized in line with the goal of producing the simplest possible model suitable for subsequent deployment in clinical practice as a simple bedside test used by rehabilitation staff within six weeks after cervical SCI. We did not use stepwise statistical variable selection procedures, such as forward inclusion or backward elimination, because this may result in biased estimates of regression coefficients and exaggeration of variable p-values. ${ }^{30-32}$ Two different single predictors were investigated: MMT subtest strength total score and SWM sensation subtest total score (with the palmar and dorsal components combined). A correlation analysis using Spearman's correlation coefficient was performed between the predictor and outcome variables of the logistic regression $\left(r_{s}\right)$ to determine the relationships between them. The level of significance was set at 0.05 . Correlations in the range of 0 to 0.25 were interpreted as none to poor, 0.26 to 0.50 as fair, 0.51 to 0.75 as moderate to good, and 0.76 to 1.0 as very good to excellent.

The performance of each model was assessed by calculating receiver operating characteristics (ROC) curves. The area under the curve (AUC) is a measure for quantifying the discriminative ability of the model. ${ }^{33}$ Values between 0.90 and 1.00 indicate excellent predictive discrimination.

Unbiased recursive partitioning is a flexible statistical model used for a variety of regression problems. A regression tool from the family of unbiased recursive partitioning methods called conditional inference tree (URP-CTREE) ${ }^{34}$ was used to produce classification trees for the outcomes QtG and self-care at 6 and 12 months, using predictors assessed between day 16 and 40 after cervical SCI. Eight different predictors were investigated: MMT strength subtest total score, MMT distal strength subtest total score, SWM (palmar and dorsal components combined) sensation subtest total score, SWM palmar sensation subtest total score, QIG subtest total score, QtG subtest total score, SCIM-SS and UEMS subtest total score. URP-CTREE creates decision rules, which divide the initial, heterogeneous patient population into increasingly homogeneous subgroups (with respect to outcome). Each rule in the classification tree is based on the singular most significant predictor, and the splits are set as to maximize discrepancy between the subsequently formed groups. The tree stops growing when there is no longer any significant predictor. The decision rules allow prediction of the response variable, and, at the same time, can be used as a stratification tool.

All data were analyzed using SPSS version 18.0 for Windows and R version 2.14.0 for Windows. 


\section{Results}

\section{Study population}

Of the 61 participants included, 56 had a traumatic and five a non-traumatic SCI. Some data was missing for four patients at the 6 month assessment and for five patients at the 12 month assessment. Injury severity and lesion level were variable. Detailed cohort characteristics are presented in Table 5.1.

Table 5.1 Demographic and clinical characteristics of participants $(n=61)$

\begin{tabular}{|c|c|}
\hline Characteristics & All participants \\
\hline \multicolumn{2}{|l|}{ Cause of $\mathrm{SCl}$} \\
\hline Traumatic & $56(91.8 \%)$ \\
\hline Non-traumatic & $5(8.2 \%)$ \\
\hline \multicolumn{2}{|l|}{ Site } \\
\hline Klinik Hohe Warte Bayreuth (D) & $20(32.8 \%)$ \\
\hline Unfallklinik Murnau (D) & $1(1.6 \%)$ \\
\hline Orthopädische Universitätsklinik Heidelberg (D) & $1(1.6 \%)$ \\
\hline Balgrist University Hospital Zurich $(\mathrm{CH})$ & $14(23 \%)$ \\
\hline Swiss Paraplegic Center Nottwil $(\mathrm{CH})$ & $25(41 \%)$ \\
\hline \multicolumn{2}{|l|}{ Gender } \\
\hline Female & $16(26.2 \%)$ \\
\hline Male & $45(73.8 \%)$ \\
\hline \multicolumn{2}{|l|}{ Age (years) } \\
\hline Median & 48 \\
\hline Mean (SD) & $46(19)$ \\
\hline $\operatorname{Min} / \max$ & $17-80$ \\
\hline \multicolumn{2}{|l|}{ AIS } \\
\hline 1 month (range $16-40$ days) $(n=61)$ & A: 16; B: 9; C: 7 ; D: 29 \\
\hline 6 months (range $150-186$ days) $(n=57)$ & A: 13; B: $7 ; C: 4 ; D: 33$ \\
\hline 12 months (range $300-400$ days) $(n=56)$ & A: 10; B: $6 ; C: 5 ; D: 35$ \\
\hline \multicolumn{2}{|l|}{ Neurological level 1 month (range $16-40$ days) } \\
\hline C1 & $3(4.9 \%)$ \\
\hline $\mathrm{C} 2$ & $6(9.8 \%)$ \\
\hline $\mathrm{C} 3$ & $11(18 \%)$ \\
\hline $\mathrm{C} 4$ & $22(36.1 \%)$ \\
\hline $\mathrm{C} 5$ & $11(18 \%)$ \\
\hline C6 & $5(8.2 \%)$ \\
\hline $\mathrm{C} 7$ & $1(1.6 \%)$ \\
\hline $\mathrm{C} 8$ & $1(1.6 \%)$ \\
\hline $\mathrm{T} 1$ & $1(1.6 \%)$ \\
\hline
\end{tabular}

Abbreviations: SCl, spinal cord injury; n, sample size; AIS, American Spinal Injury Association Impairment Scale; C2, cervical dermatome 2; $\mathrm{SD}$, standard deviation; D, Germany; $\mathrm{CH}$, Switzerland. 


\section{Spearman correlations}

The correlation between MMT total score with the outcome variables QtG and self-care at 6 and 12 months was excellent (QtG, 6 months, $r=0.885, p<0.001 ; 12$ months, $r$ $=0.904, p<0.001$; self-care, 6 months, $r=0.821, p<0.001 ; 12$ months, $r=0.820, p$ $<0.001)$. There was a moderate to good correlation between SWM total score and the outcome variables QtG and self-care at 6 and 12 months (QtG, 6 months, $r=0.651, p$ $<0.001$; 12 months, $r=0.639, p<0.001$; self-care, 6 months, $r=0.781, p<0.001 ; 12$ months, $r=0.643, p<0.001)$.

\section{Logistic regression}

For prediction of QtG and self-care outcome at 6 and 12 month based on MMT total score at 1 month, specificity ranged between $72.4 \%$ and $92.1 \%$. Sensitivity of MMT total score at 1 month ranged from $81.8 \%$ to $90.9 \%$ for the two outcomes at 6 and 12 months. In contrast, the SWM total score at 1 month performed less well with predictive specificity ranging from $69.6 \%$ to $78.9 \%$ and sensitivity from $68.2 \%$ to $84.4 \%$ at 6 and 12 months for QtG and self-care. Detailed results of the logistic regression analysis are presented in Table 5.2.

\section{ROC}

The results of the ROC analysis in predicting QtG and self-care outcome at 6 and 12 months were in line with the results of the logistic regression analysis. The AUC value for MMT was larger (ranged from 0.917 to 0.961 , p < 0.001) compared to SWM (ranged from 0.802 to $0.842, p<0.001$ ) at 6 as well as 12 months for both outcome measures. Detailed results for ROC analysis are available in Table 5.3.

\section{URP-CTREE}

We analyzed eight different predictor variables from our heterogeneous cohort of SCI patients. When these variables were placed into the recursive partitioning-based algorithm, well-defined cohorts for QtG and self-care at 6 and 12 months after cervical SCI could be distinguished. Figure 5.1a, Figure 5.1b, Figure 5.2a and Figure 5.2b show the URP-CTREE for QtG and self-care at 6 and 12 months. 


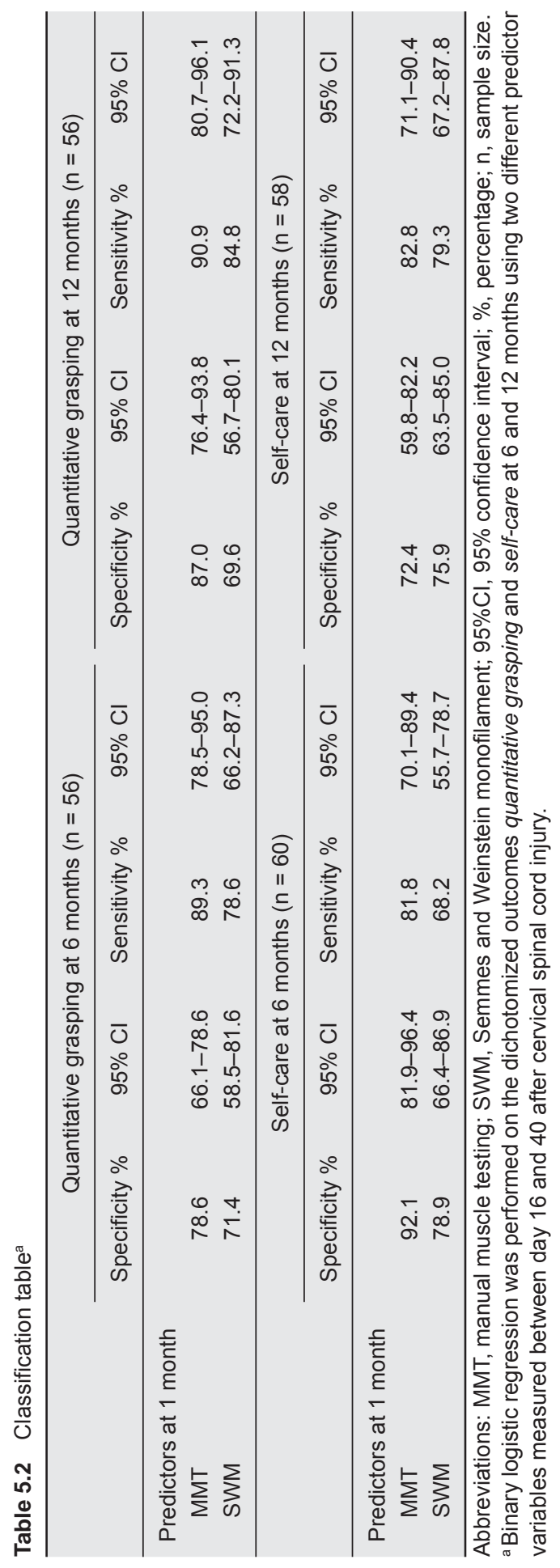




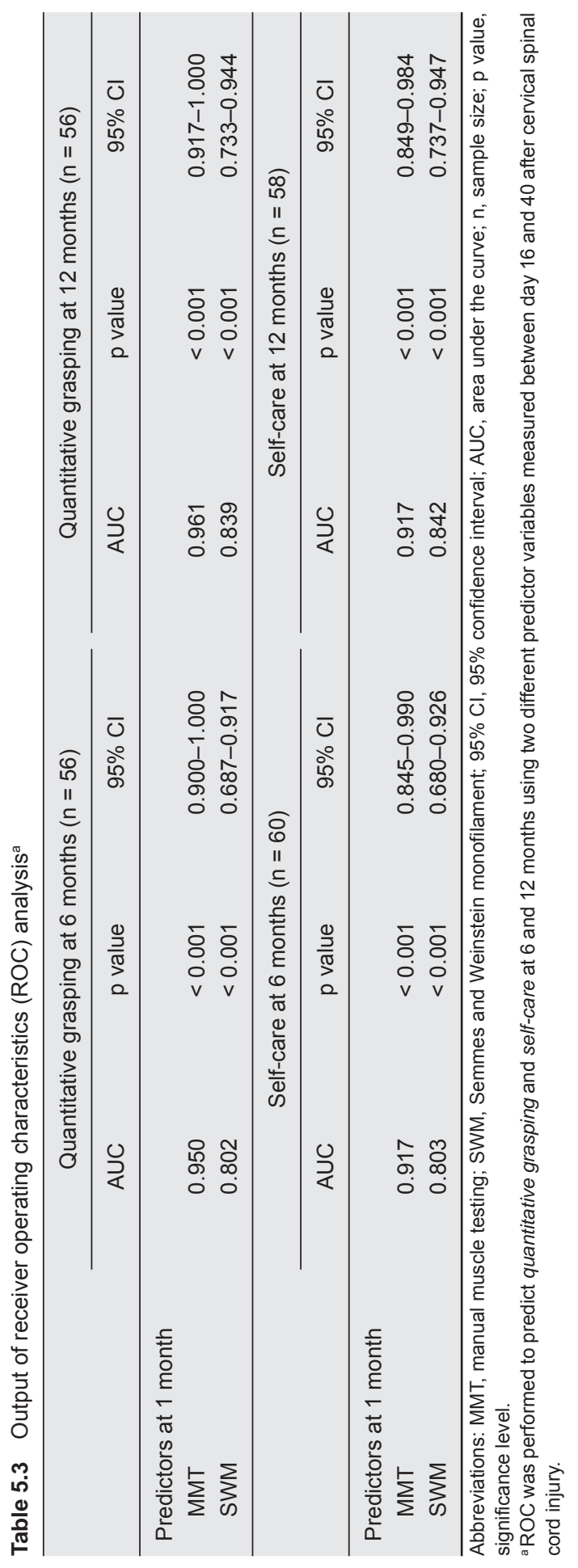

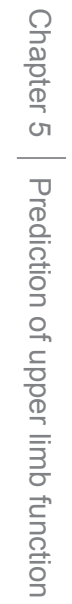



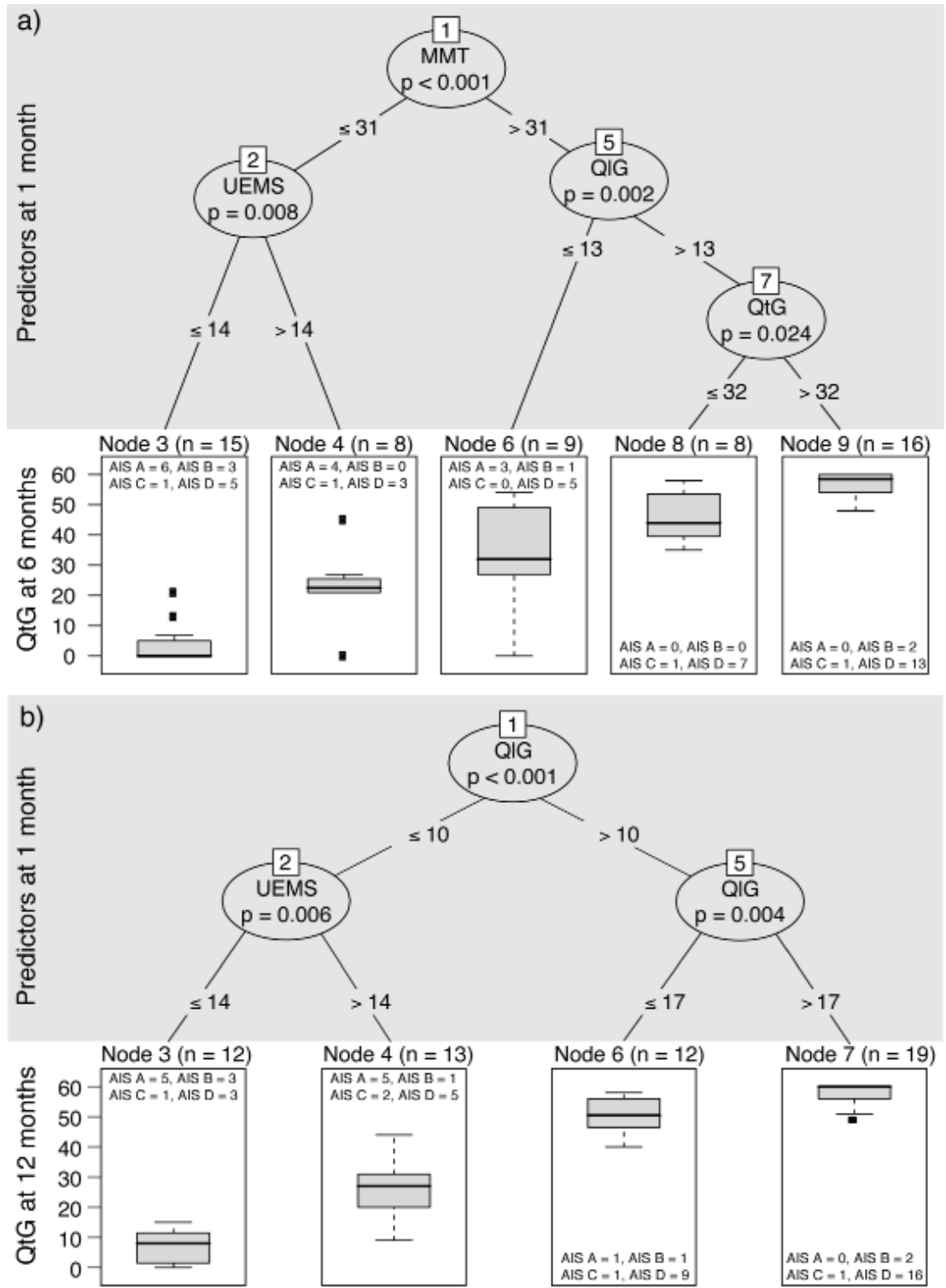

Figure 5.1 Unbiased recursive partitioning conditional inference tree (URP-CTREE) for quantitative grasping at 6 and 12 months.

(a) The algorithm led to a partition of the initial patient population into five terminal nodes. Node size is indicated above each terminal node. From left to right, the terminal nodes represent patient subgroups with an increasingly positive quantitativ grasping outcome at 6 months. The first split separates patients with an initial MMT $\leq 31$ or $>31$ as indicated by the cut-off values on the "branches". Further separation is achieved by UEMS for patients with $\leq 31$ MMT and by QIG and QtG for patients with > 31 MMT. For each inner node, a Bonferroni-adjusted p-value describing statistical association between the predictor and the outcome is given. (b) For details on the interpretation of the conditional inference tree for QtG at 12 months, please refer to the explanatory notes for "a." Abbreviations: MMT, manual muscle testing; UEMS, upper extremity motor score; QIG, qualitative grasping; QtG, quantitative grasping; AIS, American Spinal Injury Association Impairment Scale; $n$, sample size; $p$, significance level; $\leq$ less than equal to; > greater than. 

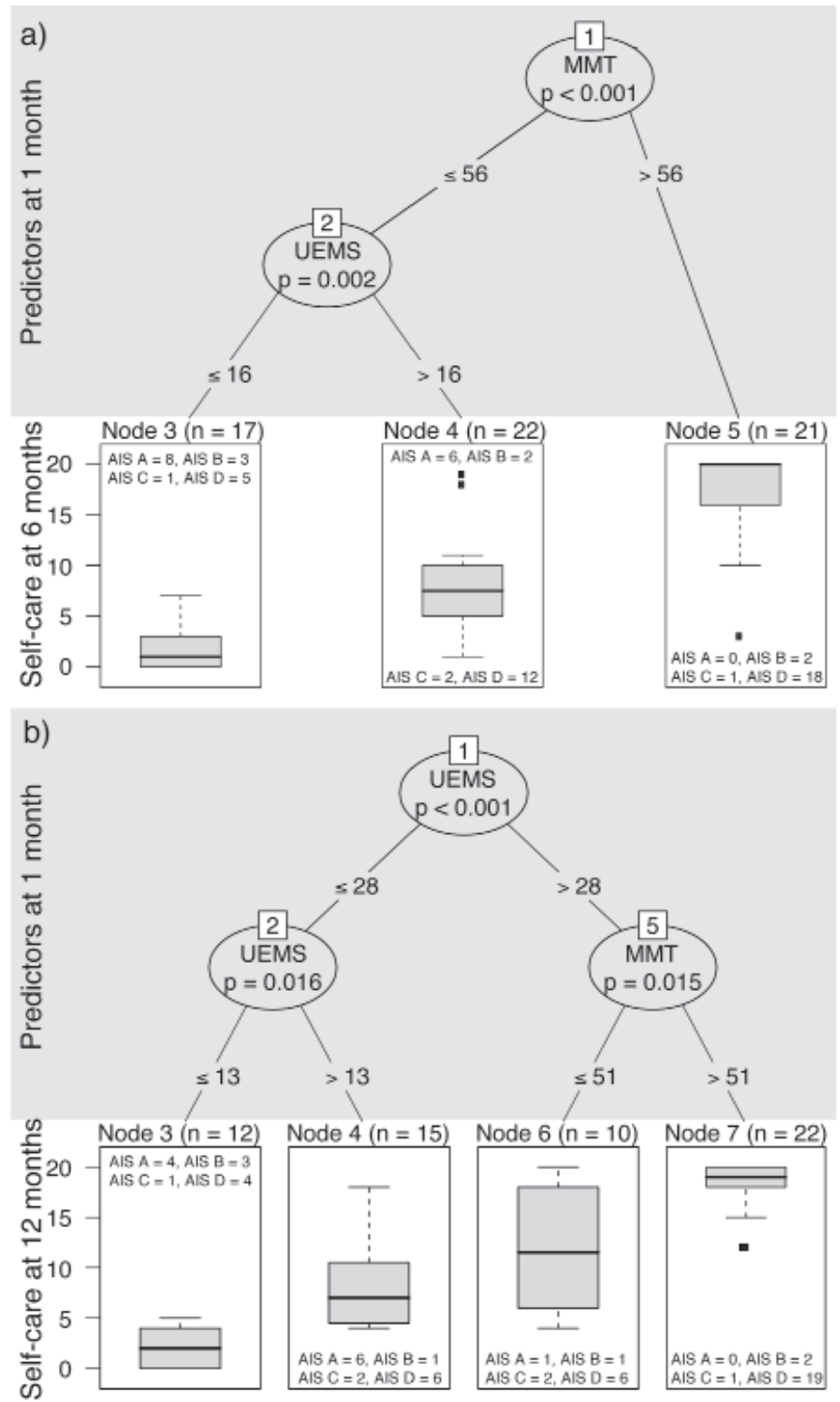

Figure 5.2 Unbiased recursive partitioning conditional inference tree (URP-CTREE) for self-care at 6 and 12 months. (a) The algorithm led to a partition of the initial patient population into three terminal nodes. Node size is indicated above each terminal node. From left to right, the terminal nodes represent patient subgroups with an increasingly positive self-care outcome at 6 months. The first split separates patients with an initial MMT $\leq 56$ or $>56$ as indicated by the cut-off values on the "branches". Further separation is achieved by UEMS for patients with $\leq 56$ MMT. For each inner node, a Bonferroni-adjusted p-value describing statistical association between the predictor and the outcome is given. (b) For details on the interpretation of the conditional inference tree for self-care at 12 months, please refer to the explanatory notes for "a".

Abbreviations: MMT, manual muscle testing; UEMS, upper extremity motor score; QIG, qualitative grasping; QtG, quantitative grasping; AIS, American Spinal Injury Association Impairment Scale; $n$, sample size; $p$, significance level; $\leq$ less than equal to; $>$ greater than. 
We now present in detail how to read the figure for QtG at 6 months (Figure 5.1a). The algorithm led to a partition of the initial sample $(n=56)$ into five terminal nodes (cohorts). The terminal nodes represent subgroups with different outcomes ranging from low to high values for $\mathrm{QtG}$ at 6 months. Initial MMT was selected as a first predictor variable ( $p$ $<0.001$ ) and separates the sample into two newly formed subgroups, MMT $\leq 31$ or $>31$ as indicated by the cut-off value at the "branches". Below this MMT $\leq 31$ subgroup ( $n=$ 23), further separation was achieved with a UEMS cut-off of 14 points ( $p=0.008)$ giving subgroups UEMS $\leq 14(n=15$; least favorable outcome) and UEMS $>14$ subgroup $(n=$ 8; second least favorable outcome). Proceeding from the MMT > 31 subgroup $(n=33)$, separation was achieved once more through the definition of a QIG cut-off of 13 points ( $p=0.002)$, giving two subgroups; $\mathrm{QIG} \leq 13(\mathrm{n}=9$; intermediate outcome) and $\mathrm{QIG}>13$ subgroup $(n=24)$. This latter grouping (QIG > 13) was further subdivided by $Q t G$ score, with a cut-off of 32 showing the largest discrepancy, again with two subgroups QtG $\leq 32$ ( $n=8$; second most favorable outcome) and QtG > 32 ( $n=16$; most favorable outcome).

\section{Discussion}

The aim of this prospective study was to evaluate the value of the GRASSP assessment tool in predicting the outcome of upper limb function and self-care at 6 and 12 months post-injury in individuals with acute cervical SCI. For this purpose, the outcome of upper limb function was assessed based on the performance of hand / upper limb activities (such as the QtG subtest) and ADLs (i.e. self-care items in the SCIM III).

The prediction of upper limb function and self-care in patients with acute cervical $\mathrm{SCl}$ can be achieved by using the GRASSP tool, of which the motor scoring in particular is of excellent predictive value for clinical outcomes at 6 and 12 months. The reliable prediction of functional outcome categories is essential for improving the stratification of patients for clinical interventions, in which the enrolment of rather homogenous patient cohorts is required. Improved stratification rules will be of benefit in trials assessing the safety and efficacy of interventions in which the detection of even subtle changes is of crucial importance in the evaluation of therapies.

\section{Clinical outcomes of upper limb function}

Given the serious consequences on patients' independence, quality of life, health care service needs and their associated socioeconomic costs, there is strong interest in the development of reliable assessment and categorization of upper limb function in tetraplegia. 
While assessments such as the Van Lieshout test (VLT) ${ }^{35}$ and the Capability of Upper Extremity Test (CUE) ${ }^{36}$ provide important information regarding overall arm and hand usage, they are not designed to provide detailed and reliable information about changes in specific sensory and motor impairments affecting upper limb function. Likewise, the utilization of a global outcome measure such as the SCIM III, although providing clinically meaningful categorization of functioning in ADLs, does not provide insights into the underlying sensorimotor function driving functional recovery. Accordingly, the SCIM III is not well positioned to discern functional improvement arising from actual repair of damaged spinal cord tissue versus rehabilitation training, motivation and mood factors when performing tasks - whether those tasks are performed bimanually or with compensatory movements given the SCIM's focus on gained independence. ${ }^{26}$

In contrast, measures designed to capture neurological deficits (e.g. the UEMS of the ISNCSCI protocol $)^{20}$ that, as opposed to functional readouts, provide detailed scoring of segmental sensorimotor deficits, have been proven to be of value in the diagnosis and prognosis of $\mathrm{SCl}$. Clinical experience, backed up by the literature, ${ }^{7,8}$ shows that recovery of upper limb function is highly variable and an assessment matrix combining neurological and functional readouts for application in acute cervical SCI may be desirable. ${ }^{13}$ To this end, the GRASSP was developed in an attempt to demonstrate how changes in impairment (i.e. neurological deficit) contribute to complex upper limb function tasks. ${ }^{15}$

\section{Prediction of upper limb function}

Analysis of a heterogeneous group of patients with acute and sub-acute cervical SCI revealed that the initial MMT correlated very well both with subsequent upper limb function and self-care at 6 and 12 months and was furthermore superior to the SWM. These findings support previous studies in which significant correlation of MMT and cervical motor levels with self-care in acute and chronic cervical SCI was shown. , $^{7,37,38}$ The strong influence of motor impairment on self-care outcomes has been shown in several studies looking at diverse functional outcome domains, including independence and ambulation. ${ }^{16,17,39-41}$ The impact of recovery of sensation, however, although shown to be critical after peripheral nerve damage, ${ }^{42}$ is of less obvious relevance in $\mathrm{SCl}$. While recordings of somatosensory evoked potentials (SSEP) correlate with the sensory impairment and have some predictive value for outcome and recovery of hand function, the immediate impact on complex arm / hand function is less marked. ${ }^{19}$ In a recent study using the GRASSP, however, it was noted that preserved sensation positively affects upper limb function, ${ }^{43}$ although the nature and degree of these relationships during the course of recovery is unknown. 
The significant role of MMT as a predictor variable with a high sensitivity and specificity in upper limb function and self-care at 6 and 12 months was also demonstrated using logistic regression and ROC. Both models corroborate the high within-sample validity of using MMT within GRASSP as predictor variable. SWM, although less influential than MMT, was also able to predict outcome of self-care and upper limb function and might be specifically applicable for prediction when motor assessments are limited (for instance when key motor muscles are not defined above C5) or when muscle activation is hindered by other factors, such as limb fractures or bruising. For the logistic regression and ROC analysis, the SCIM-SS and the GRASSP subtest QtG were converted into two dichotomous outcome measures ("dependent" vs "independent" for self-care; "non-functional” vs "functional" for grasping) which represented a wide range of upper limb performance in all subgroups. Logistic regression and ROC analysis do not, however, provide sufficient information about the distribution of outcomes.

\section{URP-CTREE}

Few studies to date have examined the stratification of outcomes of upper limb function and self-care in acute tetraplegia., ${ }^{7,8}$ For ambulation, recent studies have developed prediction rules in acute $\mathrm{SCI},{ }^{16,17,44}$ primarily based on regression analysis with the attendant, aforementioned limitation in terms of providing information about outcomes distribution. For this reason, we applied URP-CTREE to predict upper limb function and self-care as outcome measures at 6 and 12 months based on different predictor variables assessed at 1 month after injury. The results showed that URP-CTREE enables the prediction of the distribution of different outcomes in acute cervical $\mathrm{SCl}$ and the definition of more homogenous outcome cohorts. Again, in line with logistic regression and ROC analyses, MMT remained the strongest predictor for outcome of upper limb function and self-care.

MMT, as defined in the GRASSP, includes a greater number of muscles compared to the ISNCSCI protocol (UEMS) by incorporating distal (extensor digitorum, opponens pollicis, flexor pollicis longus, flexor digitorum profundus (tendon to third digit), first dorsal interosseus and abductor digiti minimi) and proximal (anterior deltoid) muscle groups. This expanded combination of distal and proximal muscle groups probably contributes to the high outcome prediction seen in this study, lending further support to the continued development of the GRASSP as a standardized assessment tool of upper limb function. Similar findings were reported in a recent review of upper extremity impairment after stroke in which it was concluded that the whole limb is important for overall function. ${ }^{45}$ 
Using URP-CTREE, we were able to show that predictors in the model demonstrated significantly differentiated predictive capacity when compared with the logistic and ROC models, including SWM and MMT as single predictors. Studies that assess the significance of combining individual parameters to improve outcome prediction are sparse. ${ }^{16,17} \mathrm{We}$ provide evidence that the combination of MMT with other predictors, such as QIG and QtG, can improve outcome prediction.

Interestingly, URP-CTREE identified UEMS as a predictor specific to individuals with less favorable functional outcomes. For patients with more favorable functional outcomes, MMT in combination with QIG and QtG demonstrated predictive utility. These data reveal that the combination of MMT strength and dexterity (QIG and QtG) interact to predict improved outcomes of upper limb function, supporting the findings of a previous study. ${ }^{46}$ The GRASSP permits the gathering of more comprehensive information (especially in motor incomplete lesions) and is capable of disentangling neurological and functional changes.

In contrast, for self-care outcomes, URP-CTREE demonstrated that the tests of muscle strength (MMT and UEMS) were useful predictor variables while QIG / QtG were not. This finding support those of a previous study which demonstrated that GRASSP subtests QIG and QtG were not superior to the muscle strength tests (UEMS and MMT) in estimating self-care independence. ${ }^{37}$

\section{Limitation}

True external validity of the proposed prediction models can only be proven through confirmatory analysis of an independent data set. Many clinical assessments like UEMS, SCIM and GRASSP are analyzed as sum scores of different items and treated as continuous variables, even though they are ordinal scales. We acknowledge that this could produce misleading results where summed scores do not represent a consistent scoring metric.

\section{Conclusion}

The GRASSP is a feasible and reliable assessment tool for the prediction of upper limb function and self-care outcomes in individuals with acute cervical SCI. The GRASSP at 1 month can accurately predict functional outcome at 6 and 12 months, even in a heterogeneous group of individuals across a wide spectrum of neurological recovery. Prediction of outcomes can be used to inform rehabilitation goals and regimens and can be applied in improved stratification of patients in evaluation of interventions. The additional 
application of URP-CTREE permits insights into the distribution of outcome categories on which clinical trial outcome analysis and stratification may be based.

\section{Acknowledgments}

We thank the patients with SCI who participated in this study and occupational therapists at the participating centers: Spinal Cord Injury Center, Hohe Warte, Bayreuth, Germany; Orthopädische Universitätsklinik Heidelberg, Germany; Unfallklinik Murnau, Germany; Balgrist University Hospital, Zurich, Switzerland and the Swiss Paraplegic Center, Nottwil, Switzerland. Furthermore we thank Dr Aurelio Tobón (Nottwil) for performing the clinical neurological examinations, Mr Tim Killeen (Balgrist) for critically reviewing the manuscript and his invaluable assistance with English language editing and Dr Doris Maier (Murnau) and Dr Rüdiger Rupp (Heidelberg) for their collaboration on this project.

\section{Funding}

The study was supported by the Swiss Paraplegic Center, Nottwil, Switzerland and funded by the NeuroRehab Clinical Research Priority Program at the University of Zurich, Switzerland.

\section{References}

1. Anderson KD. Targeting recovery: priorities of the spinal cord-injured population. J Neurotrauma 2004;21:1371-1383.

2. Snoek GJ, IJzerman MJ, Hermens HJ, Maxwell D, Biering-Sorensen F. Survey of the needs of patients with spinal cord injury: impact and priority for improvement in hand function in tetraplegics. Spinal Cord 2004;42:526-532.

3. Curt A, Schwab ME, Dietz V. Providing the clinical basis for new interventional therapies: refined diagnosis and assessment of recovery after spinal cord injury. Spinal Cord 2004;42:1-6.

4. Fawcett JW, Curt A, Steeves JD, et al. Guidelines for the conduct of clinical trials for spinal cord injury as developed by the ICCP panel: spontaneous recovery after spinal cord injury and statistical power needed for therapeutic clinical trials. Spinal Cord 2007;45:190-205.

5. Geisler FH, Coleman WP, Grieco G, Poonian D. Measurements and recovery patterns in a multicenter study of acute spinal cord injury. Spine (Phila Pa 1976) 2001;26:S68-86.

6. Ragnarsson KT, Wuermser LA, Cardenas DD, Marino RJ. Spinal cord injury clinical trials for neurologic restoration: improving care through clinical research. Am J Phys Med Rehabil 2005;84:S77-97; quiz S98-100.

7. Kramer JL, Lammertse DP, Schubert M, Curt A, Steeves JD. Relationship between motor recovery and independence after sensorimotor-complete cervical spinal cord injury. Neurorehabil Neural Repair 2012;26:1064-1071. 
8. Steeves JD, Lammertse DP, Kramer JL, et al. Outcome Measures for Acute/Subacute Cervical Sensorimotor Complete (AIS-A) Spinal Cord Injury During a Phase 2 Clinical Trial. Top Spinal Cord Inj Rehabil 2012;18:1-14.

9. Schonherr MC, Groothoff JW, Mulder GA, Eisma WH. Prediction of functional outcome after spinal cord injury: a task for the rehabilitation team and the patient. Spinal Cord 2000;38:185191.

10. Velstra IM, Ballert CS, Cieza A. A systematic literature review of outcome measures for upper extremity function using the international classification of functioning, disability, and health as reference. PM R 2011;3:846-860.

11. van Tuijl JH, Janssen-Potten YJ, Seelen HA. Evaluation of upper extremity motor function tests in tetraplegics. Spinal Cord 2002;40:51-64.

12. Boakye M, Harkema S, Ellaway PH, Skelly AC. Quantitative testing in spinal cord injury: overview of reliability and predictive validity. Journal of neurosurgery Spine 2012;17:141-150.

13. Aidinoff E, Benjamini $Y$, Galili $T$, et al. Non-linear formulas for the spinal cord injury ability realization measurement index. Spinal Cord 2012;50:324-327.

14. Kalsi-Ryan S, CurtA, Fehlings MG, Verrier MC. Assessment of the Hand in Tetraplegia Using the Graded Redefined Assessment of Strength, Sensibility and Prehension (GRASSP): Impairment Versus Function. Top Spinal Cord Inj Rehabil 2009;14:34-46.

15. Kalsi-Ryan S, Beaton D, Curt A, et al. The Graded Redefined Assessment of Strength Sensibility and Prehension (GRASSP): Reliability and Validity. J Neurotrauma 2011.

16. van Middendorp JJ, Hosman AJ, Donders AR, et al. A clinical prediction rule for ambulation outcomes after traumatic spinal cord injury: a longitudinal cohort study. Lancet 2011;377:10041010.

17. Zorner B, Blanckenhorn WU, Dietz V, Curt A. Clinical algorithm for improved prediction of ambulation and patient stratification after incomplete spinal cord injury. J Neurotrauma 2010;27:241-252.

18. Wilson JR, Grossman RG, Frankowski RF, et al. A clinical prediction model for long-term functional outcome after traumatic spinal cord injury based on acute clinical and imaging factors. J Neurotrauma 2012;29:2263-2271.

19. Curt A, Dietz V. Traumatic cervical spinal cord injury: relation between somatosensory evoked potentials, neurological deficit, and hand function. Arch Phys Med Rehabil 1996;77:48-53.

20. Kirshblum SC, Waring W, Biering-Sorensen F, et al. Reference for the 2011 revision of the International Standards for Neurological Classification of Spinal Cord Injury. J Spinal Cord Med 2011;34:547-554.

21. Wright JG, Feinstein AR. Improving the reliability of orthopaedic measurements. J Bone Joint Surg Br 1992;74:287-291.

22. Schuld C, Wiese J, Hug A, et al. Computer implementation of the international standards for neurological classification of spinal cord injury for consistent and efficient derivation of its subscores including handling of data from not testable segments. J Neurotrauma 2012;29:453461.

23. Daniels L, Worthingham C. Daniels and Worthingham's Muscle Testing: Techniques of Manual Examination, 6th ed. Washington, 1995.

24. Mackin E, Callahan A, Skiver T, Schneider L, Osterman A. Hunter-Mackin Callahan Rehabilitation of the Hand and Upper Extremity, 5th ed. St. Louis: Mosby, 2003. 
25. Sollerman C, Ejeskar A. Sollerman hand function test. A standardised method and its use in tetraplegic patients. Scandinavian journal of plastic and reconstructive surgery and hand surgery / Nordisk plastikkirurgisk forening [and] Nordisk klubb for handkirurgi 1995;29:167-176.

26. Catz A, Itzkovich M, Agranov E, Ring H, Tamir A. SCIM--spinal cord independence measure: a new disability scale for patients with spinal cord lesions. Spinal Cord 1997;35:850-856.

27. Anderson KD, Acuff ME, Arp BG, et al. United States (US) multi-center study to assess the validity and reliability of the Spinal Cord Independence Measure (SCIM III). Spinal Cord 2011;49:880-885.

28. Catz A, Itzkovich M, Tesio L, et al. A multicenter international study on the Spinal Cord Independence Measure, version III: Rasch psychometric validation. Spinal Cord 2007;45:275291.

29. Itzkovich M, Gelernter I, Biering-Sorensen F, et al. The Spinal Cord Independence Measure (SCIM) version III: reliability and validity in a multi-center international study. Disabil Rehabil 2007;29:1926-1933.

30. Murray GD, Butcher I, McHugh GS, et al. Multivariable prognostic analysis in traumatic brain injury: results from the IMPACT study. J Neurotrauma 2007;24:329-337.

31. Harrel F. Regression Modeling Strategies with Applications to Linear Models, Logistic Regression and Survival Analysis. New York: Springer Verlag, 2001

32. Steyerberg E. Clinical Prediction Models: A Practical Approach to Development, Validation, and Updating. New York: Springer, 2009.

33. Hanley JA. A method of comparing the areas under receiver operating characteristic curves derived from the same cases. Radiology 1983;148:839-843.

34. Hothorn T, Hornik K, Zeileis A. Unbiased recursive partitioning: a conditional inference framework. J Comput Graph Stat 2006;15:651-674.

35. Post MW, Van Lieshout G, Seelen HA, Snoek GJ, ljzerman MJ, Pons C. Measurement properties of the short version of the Van Lieshout test for arm/hand function of persons with tetraplegia after spinal cord injury. Spinal Cord 2006;44:763-771.

36. Marino RJ, Patrick M, Albright W, et al. Development of an objective test of upper-limb function in tetraplegia: the capabilities of upper extremity test. Am J Phys Med Rehabil 2012;91:478486.

37. Rudhe C, van Hedel HJ. Upper extremity function in persons with tetraplegia: relationships between strength, capacity, and the spinal cord independence measure. Neurorehabil Neural Repair 2009;23:413-421.

38. van Hedel HJ, Curt A. Fighting for each segment: estimating the clinical value of cervical and thoracic segments in SCI. J Neurotrauma 2006;23:1621-1631.

39. Curt A, Keck ME, Dietz V. Functional outcome following spinal cord injury: significance of motor-evoked potentials and ASIA scores. Arch Phys Med Rehabil 1998;79:81-86.

40. Marino RJ, Rider-Foster D, Maissel G, Ditunno JF. Superiority of motor level over single neurological level in categorizing tetraplegia. Paraplegia 1995;33:510-513.

41. Waters RL, Adkins RH, Yakura JS, Sie I. Motor and sensory recovery following incomplete tetraplegia. Arch Phys Med Rehabil 1994;75:306-311.

42. Rosen B, Dahlin LB, Lundborg G. Assessment of functional outcome after nerve repair in a longitudinal cohort. Scandinavian journal of plastic and reconstructive surgery and hand surgery / Nordisk plastikkirurgisk forening [and] Nordisk klubb for handkirurgi 2000;34:71-78. 
43. Kalsi-Ryan S, Wilson J, Yang JM, Fehlings M. Neurologic Grading in Traumatic Spinal Cord Injury. World neurosurgery, 2013.

44. Wilson JR, Arnold PM, Singh A, Kalsi-Ryan S, Fehlings MG. Clinical prediction model for acute inpatient complications after traumatic cervical spinal cord injury: a subanalysis from the Surgical Timing in Acute Spinal Cord Injury Study. Journal of Neurosurgery Spine 2012;17:46-51.

45. Lang CE, Bland MD, Bailey RR, Schaefer SY, Birkenmeier RL. Assessment of upper extremity impairment, function, and activity after stroke: foundations for clinical decision making. J Hand Ther 2013;26:104-114;quiz 115.

46. Zariffa J, Kapadia N, Kramer JL, et al. Feasibility and efficacy of upper limb robotic rehabilitation in a subacute cervical spinal cord injury population. Spinal Cord 2012;50:220-226. 



\title{
Predictive value of upper limb muscles and grasp patterns on functional outcome in cervical spinal cord injury
}

\author{
Inge-Marie Velstra \\ Marc Bolliger \\ Jörg Krebs \\ Johan S. Rietman \\ Armin Curt
}

Neurorehabil Neural Repair 2015; in press Copyright (C) (2015) by The American Society of Neurorehabilitation. Reprinted by permission of SAGE Publications, Inc. 


\section{Abstract}

Objective: To determine which single or combined upper limb muscles as defined by the International Standards for the Neurological Classification of Spinal Cord Injury (ISNCSCI); upper extremity motor score (UEMS) and the Graded Redefined Assessment of Strength, Sensibility and Prehension (GRASSP), best predict upper limb function and independence in activities of daily living (ADLs) and to assess the predictive value of qualitative grasp movements (QIG) on upper limb function in individuals with acute tetraplegia.

Method: As part of a Europe-wide, prospective, longitudinal, multicenter study ISNCSCI, GRASSP and Spinal Cord Independence Measure (SCIM III) scores were recorded at 1 and 6 months after SCI. For prediction of upper limb function and ADLs, a logistic regression model and unbiased recursive partitioning conditional inference tree (URPCTREE) were used.

Results: Logistic regression and URP-CTREE revealed that a combination of ISNCSCI and GRASSP muscles (to a maximum of four) demonstrated the best prediction (specificity and sensitivity ranged from $81.8 \%$ to $96.0 \%$ ) of upper limb function and identified homogenous outcome cohorts at 6 months. The URP-CTREE model with the QIG predictors for upper limb function showed similar results.

Conclusion: Prediction of upper limb function can be achieved through a combination of defined, specific upper extremity muscles assessed in the ISNCSCI and GRASSP. A combination of a limited number of proximal and distal muscles along with an assessment of grasping movements can be applied for clinical decision making for rehabilitation interventions and clinical trials. 


\section{Introduction}

The neurological examination of individuals with spinal cord injury ( $\mathrm{SCl}$ ) is usually performed according to the International Standards for the Neurological Classification of Spinal Cord Injury (ISNCSCI), ${ }^{1}$ which is considered the gold standard to determine the levels of injury and to classify the severity of injury. The upper extremity motor score (UEMS, Table 6.1), a component of the ISNCSCI, is often used in clinical research to examine the course of spontaneous neurological recovery. ${ }^{2-5}$ However, the UEMS is limited to the assessment of only five key muscle groups for each upper limb in SCl. Furthermore, individuals with cervical $\mathrm{SCI}$ show a high variability in motor recovery, ${ }^{4,6-8}$ following acute injury. Therefore, the sensitivity of the UEMS is limited for both the prediction and measurement of a therapeutic benefit (i.e. suffering from floor and ceiling effects). Accordingly, the ISNCSCI worksheet was recently updated ${ }^{9}$ with the description

Table 6.1 Abbreviations

\begin{tabular}{|c|c|}
\hline ISNCSCI & International Standards for Neurological Classification of Spinal Cord Injury \\
\hline ASIA & American Spinal Injury Association \\
\hline AIS & American Spinal Injury Association Impairment Scale \\
\hline MLI & Motor level of injury \\
\hline UEMS & Upper extremity motor score \\
\hline ElbowFlex & Elbow flexors \\
\hline WristExt & Wrist extensors \\
\hline Triceps & Elbow extensors \\
\hline FDP & Long finger flexors \\
\hline AbdDigV & Small finger abductors \\
\hline SCIM & Spinal Cord Independence Measure \\
\hline SCIM-SS & Spinal Cord Independence Measure; self-care subscale \\
\hline SCIM-Mobs & Spinal Cord Independence Measure; mobility subscale \\
\hline GRASSP & Graded Redefined Assessment of Strength, Sensibility and Prehension \\
\hline MMT & Manual muscle testing \\
\hline Delto & M. anterior deltoid \\
\hline EDC & M. extensor digitorum communis \\
\hline OPP & M. opponens pollicis \\
\hline FPL & M. flexor pollicis longus \\
\hline DI1 & M. first dorsal interosseus \\
\hline SWM & Semmes and Weinstein monofilament \\
\hline QIG & Qualitative grasping \\
\hline CylGrasp & Cylindrical grasp \\
\hline LatPinch & Lateral key pinch \\
\hline TTPinch & Tip-to-tip pinch \\
\hline QtG & Quantitative grasping \\
\hline
\end{tabular}


of additional non-key muscle functions for the upper and lower extremities although their sensitivity of prediction and responsiveness has not yet been analysed.

With the intention of providing more sensitive and accurate assessments of upper limb recovery in cervical SCI, the Graded Redefined Assessment of Strength, Sensibility and Prehension (GRASSP) was developed by an international research group. ${ }^{10}$ The GRASSP has strong potential to improve the current clinical assessments of upper limb function ${ }^{6,11}$ since it consists of different comprehensive subtests (i.e. assesses an increased number of upper limb muscles) and has demonstrated excellent psychometric properties. ${ }^{6,12}$

In recent years there have been several investigations into the prediction of functional outcomes after $\mathrm{SCl},{ }^{13-16}$ increasing our knowledge of recovery and prediction of upper limb function and self-care after cervical SCI.6,7,17,18 The GRASSP allows the precise identification of recovery profiles ${ }^{6}$ and accurately predicts upper limb function and selfcare in acute tetraplegia. ${ }^{7}$

The GRASSP strength subtest assesses ten muscles, of which five are also measured in ISNCSCI-UEMS (please see Table 6.2). The number of muscles to be tested should be kept to a minimum and should only include muscles that are useful in the clinical setting and for research in order to optimise time resources. However, there is insufficient evidence regarding the influence of each individual muscle or muscle group on the prediction of upper limb function and activities of daily living (ADLs). Furthermore, the significance of specific grasp movements presenting early after injury and their potential predictive value for functional outcomes has not been studied so far. These specific grasp movements represent complex outcomes (i.e. combined sensory and motor outputs) that may classify impairment and neurological deficit of the hand more comprehensively than isolated motor and sensory scores. This prospective study on acute cervical $\mathrm{SCl}$ up to 6 months thus addressed two aims; firstly, to determine which single or combined upper limb muscles as defined by the ISNCSCI-UEMS and the GRASSP, best predict upper limb function and independence in ADLs, and secondly, to assess the predictive value of qualitative grasp movements (QIG) on upper limb function.

\section{Methods}

Study design

This is a European prospective longitudinal multicenter study. 


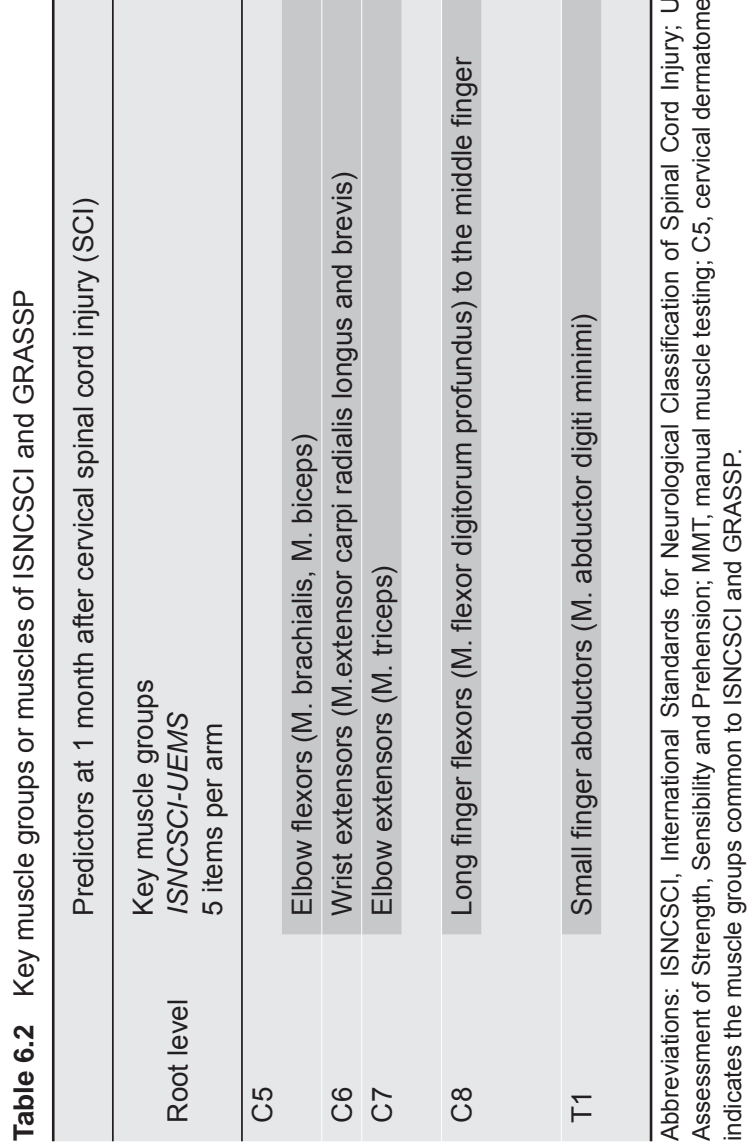




\section{Study population}

Individuals with acute tetraplegia were prospectively enrolled in five European SCI centers from 2009 to 2012. Individuals were included if they had sustained a traumatic or nontraumatic acute (16-40 days after injury) cervical spinal cord injury as defined by the protocol of the American Spinal Injury Association (ASIA ${ }^{1}$ and suffered from any Impairment Scale (AIS) grade of A, B, C or D. Participants with high cervical lesions, continuous complete ventilator dependency and complete loss of upper limb control were excluded. Participants were also excluded if they had any accompanying severe neurological disorder (e.g., traumatic brain injury), any additional cause of upper limb impairment or were younger than 16 years old. The study was approved by relevant authorities at all sites and written informed consent was obtained from all participants.

\section{Clinical assessments and procedures}

The main clinical measures included the ISNCSCI, GRASSP and the Spinal Cord Independence Measure (SCIM).

The clinical neurological examination was performed according to the ISNCSCI protocol, ${ }^{1}$ which is the gold standard to determine the levels of injury and to classify the severity of the injury. This paper focuses on subcomponents of the ISNCSCI, the UEMS, the motor level of injury (MLI) and AIS.

The GRASSP is a comprehensive measure of upper limb function with motor (manual muscle testing (MMT)), sensory (Semmes and Weinstein monofilament (SWM)), qualitative grasping (QIG) and quantitative grasping (QtG) subtests. The right and left sides are tested separately. The subtests and items within subtests can be evaluated separately or as summed scores. The GRASSP is a relatively new tool, which is being used more and more commonly in clinical research of cervical SCI and has excellent psychometric properties. ${ }^{6,12}$ More details about the GRASSP Version 1.0 are described elsewhere. ${ }^{6,7}$

The SCIM III is a global measure of function which assesses independence in fundamental daily activities specific to individuals with SCI. ${ }^{19}$ The SCIM III consists of three subcategories: (a) self-care (SCIM-SS), (b) respiration and sphincter management, and (c) mobility (SCIM-MobS). The SCIM III has been shown to perform well under psychometric testing. ${ }^{20-22}$

Rehabilitation physicians trained on the ISNCSCI protocol performed the neurological examination and occupational therapists conducted the GRASSP at 1 month (range $=16-40$ days) and 6 months (range $=150-186$ days) after cervical SCI. All occupational therapists 
involved in data collection had at least one year of experience in working with individuals with $\mathrm{SCl}$ and had successfully completed competency training on how to perform the outcome measure assessments. Experienced physical therapists, nurses and occupational therapists obtained SCIM III data at 6 months (range = 150-186 days) after cervical SCI. In addition, a standard protocol, outlining in detail how the assessments should be performed, was provided for each outcome measure with standardised recording techniques and materials across all centers.

\section{Predictor variables}

The following baseline variables (assessed between day 16 and 40 after cervical SCI) were selected as predictors: INSCSCI-AIS, INSCSCI-MLI, ISNCSCI-UEMS, GRASSP-MMT and GRASSP-QIG.

ISNCSCI-UEMS. The ISNCSCI upper limb strength measurement consists of five key muscle groups. Each muscle group is scored from 0 (completely paralysed muscle) to 5 (active movement and a full range of movement against maximum resistance) for each arm.

GRASSP-MMT. GRASSP strength measurement consists of ten muscles. Each muscle is scored from 0 (completely paralysed muscle) to 5 (active movement and a full range of movement against maximum resistance) for each arm. For this study, the five muscles which are also assessed in the UEMS were excluded from analysis. The five remaining muscles were selected as predictors. For more details regarding the ISNCSCI and GRASSP predictor variables, please see Table 6.2.

GRASSP-QIG. The ability to perform movements of the hand and fingers as they relate to a cylindrical grasp (CylGrasp), lateral key pinch (LatPinch), and tip-to-tip pinch (TTpinch) is assessed for each hand separately. Each grasp is scored from 0 (no voluntary control of wrist and digits when grasping) to 4 (normal voluntary movement control of wrist and digits when generating the grasp).

INSCSCI-AIS and INSCSCI-MLI. The AIS classifications were calculated using a computer algorithm ${ }^{23}$ in accordance with the definitions in the ISNCSCI. ${ }^{1}$ As described in the ISNCSCI, the motor level is defined as the most caudal spinal segment, as indexed by the key muscle group for that segment, having a muscle strength score of at least $3 / 5$ (full range contraction against gravity alone) while all the more rostral key muscles are normal (5/5). The MLI was split in three subgroups: 1) C1-C4; 2) C5-C6; 3) C7-T1. 


\section{Outcome variables}

The appreciation of upper limb function was distinguished into different domains like the quantitative capacity of each single hand to accomplish defined grasping tasks (QtG) and defined measures of independence in activities of daily life (SCIM items). All the outcome variables were assessed at 6 months (range $=150-186$ days) after injury.

GRASSP-QtG. The GRASSP subtest QtG reflects upper limb function based on quantitative measures of grasp performance. Six prehension tasks were performed in a standardised way. Each task is graded from 0 (the task cannot be conducted at all) to 5 (the task is conducted without difficulties using the expected grasping pattern and upper limb function is unaffected) for each arm according to the grasp used and completeness of the task within 75 seconds. The scores of the six tasks were added, giving a maximum possible QtG subtest score of 30 points for each side. The scoring was performed according to the GRASSP protocol.

SCIM III. The SCIM-SS and SCIM-MobS were selected as outcome variables because these subcategories have items predominantly related to the use of the upper limb (transfers, wheelchair mobility, grooming etc.) and reflect upper limb performance. ${ }^{24}$ The sum of the SCIM-SS ranges from 0 to 20 points. The SCIM-MobS is the sum of the SCIM mobility subcategory minus the score for the "stair" item. The sum of this SCIMMobs therefore ranges from 0 to 37 points.

\section{Data analysis}

Descriptive statistics were used to determine the frequency, mean and range of the study individuals' characteristics including AIS grade, motor level, gender and age.

Backward multiple binary logistic regression was performed to identify the muscle and muscle group variables that show the greatest effect on the prediction of the dichotomised outcomes QtG, SCIM-SS and SCIM-MobS. The following five ISNCSCI-UEMS muscle group predictors were investigated for each arm: elbow flexors (ElbowFlex), wrist extensors (WristExt), elbow extensors (Triceps), long finger flexors (FDP) and small finger abductors (AbdDigV). The five GRASSP single muscle predictors that are not included in the UEMS muscle groups were investigated in this study: M. anterior deltoid (Delto), M. extensor digitorum communis (EDC), M. opponens pollicis (Opp), M. flexor pollicis longus (FPL) and M. first dorsal intersosseus (DI1) (please see Table 6.1 and Table 6.2 for details). Ten muscles or muscle group predictor variables were therefore included in the model and were subsequently eliminated in a backward stepwise regression method using the Likelihood-Ratio (LR) test. 
Sensitivity and specificity were calculated from classification tables with $95 \%$ confidence intervals (Cls), to investigate the predictive accuracy of the full and reduced model. Dichotomisation of the QtG and SCIM-SS was based on known cutoff values described elsewhere. ${ }^{7}$ Briefly, for the unilateral QtG outcome, individuals were allocated to the success group (19-30 points) if they were able to complete the task using the standard grasp, irrespective of any difficulties while performing the task. All other individuals with scores below 19 points were allocated to the failure group. A cutoff SCIM-SS score of 12 was applied, with scores of 0 to 12 points defined as dependent (failure), and scores from 13 to 20 as independent (success). Dichotomisation of the SCIM-MobS was based on those items that characterised that individuals would need total or partial assistance and/or adaptive devices in mobility activities and are referred to as dependent in mobility (failure) while the other group consisted of individuals that are referred to as independent (success) in mobility. A SCIM-MobS score from 0 to 12 points was therefore defined as failure, and a score from 13 to 37 was defined as success.

We furthermore based our analysis on a flexible tree-structured regression model from the family of unbiased recursive partitioning methods called conditional inference tree (URPCTREE) ${ }^{25}$ which is an unbiased technique to directly identify homogenous subgroups without compromising prediction accuracy in $\mathrm{SCl} .7,26$ We generated classification trees for the outcomes QtG, SCIM-SS and SCIM-MobS at 6 months using the same ten muscle and muscle group predictors as described under the logistic regression section, assessed at 1 month after cervical SCI. In addition to the 10 muscle predictors the two ISNCSCI predictors AIS and MLI were entered in the model. URP-CTREE does not assume linear dependence between predictors and outcomes, and it specifically puts the modelling focus on interactions between predictors. Each decision in the classification tree is based on the singular most significant predictor, and the splits are set as to maximise discrepancy between the groups subsequently formed. The tree stops growing when there is no longer any significant predictor. The same statistics were performed for each hand with the three QIG predictors: CylGrasp, LatPinch and TTpinch. For the unilateral QtG (30 points maximum sum score) outcome variable, the unilateral predictor variable scores (ordinal range from 0 to 5 for strength and 0 to 4 for QIG) were used in the analysis. For the SCIM-SS (20 points maximum summed score) and SCIM-MobS (maximum summed score 37 points) outcome variables, the right and left predictor variables were combined, giving a bilateral predictor variable score (10 points maximum sum score). All data were analysed using SPSS (IBM) version 18.0 for Windows and the computing environment $R^{27}$ version 2.14.0 for Windows which based on the package "party: a laboratory for recursive partitioning". ${ }^{28}$ 


\section{Results}

\section{Study population}

A total of 61 individuals with cervical $\mathrm{SCl}$ were enrolled in the study. For four individuals, no QtG data was available at 6 months and therefore a total of 57 individuals $(n=114$ arms) were included for the GRASSP outcome QtG and 61 individuals for the SCIM-SS and SCIM-MobS outcomes. The mean age of the included participants was 47 ( \pm 19 SD;

Table 6.3 Demographic and clinical characteristics of participants $(n=61)$

\begin{tabular}{|c|c|c|}
\hline Characteristics & \multicolumn{2}{|l|}{$\mathrm{n}(\%)$} \\
\hline \multicolumn{3}{|l|}{ Cause of SCl } \\
\hline Traumatic & \multicolumn{2}{|l|}{$58(95.1 \%)$} \\
\hline Non-traumatic & \multicolumn{2}{|l|}{$3(4.9 \%)$} \\
\hline \multicolumn{3}{|l|}{ Site } \\
\hline Klinik Hohe Warte Bayreuth (D) & \multicolumn{2}{|l|}{$19(31.1 \%)$} \\
\hline Unfallklinik Murnau (D) & \multicolumn{2}{|l|}{$1(1.6 \%)$} \\
\hline Orthopädische Universitätsklinik Heidelberg (D) & \multicolumn{2}{|l|}{$3(4.9 \%)$} \\
\hline Balgrist University Hospital Zurich $(\mathrm{CH})$ & \multicolumn{2}{|l|}{$13(31.1 \%)$} \\
\hline Swiss Paraplegic Center Nottwil $(\mathrm{CH})$ & \multicolumn{2}{|l|}{$25(41.0 \%)$} \\
\hline \multicolumn{3}{|l|}{ Gender } \\
\hline Female & \multicolumn{2}{|l|}{$16(26.2 \%)$} \\
\hline Male & \multicolumn{2}{|l|}{$45(73.8 \%)$} \\
\hline Age (mean years; SD; min/max) & \multicolumn{2}{|c|}{$47( \pm 19 ; 18-81)$} \\
\hline \multicolumn{3}{|l|}{ AIS } \\
\hline 1 month (range $16-40$ days) $(n=61)$ & \multicolumn{2}{|c|}{$\begin{array}{l}\text { A } 16(26.2 \%) \text {; B } 10(16.4 \%) \text {; } \\
\text { C } 7(11.5 \%) \text {; D } 28(45.9 \%)\end{array}$} \\
\hline 6 months (range $150-186$ days) $(n=61)$ & \multicolumn{2}{|c|}{$\begin{array}{l}\text { A } 14(23.0 \%) \text {; B } 7(11.5 \%) \text {; } \\
\text { C } 4(6.6 \%) ; \text { D } 36(59.0 \%)\end{array}$} \\
\hline Motor level of injury at 1 and 6 months & 1 month & 6 months \\
\hline C1 & $3(4.9 \%)$ & $2(3.3 \%)$ \\
\hline $\mathrm{C} 2$ & $4(6.6 \%)$ & $5(8.2 \%)$ \\
\hline $\mathrm{C} 3$ & $13(21.3 \%)$ & $8(13.1 \%)$ \\
\hline $\mathrm{C} 4$ & $9(14.8 \%)$ & $3(4.9 \%)$ \\
\hline C5 & $14(23.0 \%)$ & $15(24.6 \%)$ \\
\hline C6 & $8(13.1 \%)$ & $10(16.4 \%)$ \\
\hline $\mathrm{C} 7$ & $5(8.2 \%)$ & $8(13.1 \%)$ \\
\hline $\mathrm{C} 8$ & $3(4.9 \%)$ & $7(11.5 \%)$ \\
\hline $\mathrm{T} 1$ & $2(3.3 \%)$ & $2(3.3 \%)$ \\
\hline $\mathrm{T} 2$ & & $1(1.6 \%)$ \\
\hline
\end{tabular}

Abbreviations: $\mathrm{n}$, sample size; SCl, spinal cord injury; AIS, American Spinal Injury Association Impairment Scale; C1, cervical dermatome 1; C, cervical; T1, thoracic dermatome 1; T, thoracic; dermatomes are indicated by numbers; SD, standard deviation; D, Germany; $\mathrm{CH}$, Switzerland. 
18-81) years and $45(73.8 \%)$ of the individuals were male. Detailed cohort characteristics are presented in Table 6.3.

\section{Logistic regression}

Completion of logistic regression analyses based on the previous defined binary outcome variables (see Methods) in general revealed that the best statistical models did not reduce prediction accuracy (represented by the high sensitivity and specificity levels) compared to the full model with ten muscle predictors included. The observed predictions for the full and reduced models are presented in Table 6.4.

Backward logistic regression (LR) identified the combination of ISNCSCI-FDP ( $p<$ $0.0001)$ and GRASSP-Delto $(p<0.014)$ as the best statistical model for binary outcome in QtG. For the prediction of the SCIM-SS in categorising individuals as dependent or independent in self-care, the combination of two ISNCSCI muscle predictors (ElbowFlex ( $p$ $<0.0001)$ and WristExt $(p<0.027))$ and two GRASSP muscle predictors (EDC $(p<0.028)$ and FPL $(p<0.001)$ was found as the most accurate prediction model. The combination of two ISNCSCI muscle predictors (WristExt $(p<0.047)$ and FDP $(p<0.065))$ and two GRASSP muscle predictors (Delto $(p<0.006)$ and FPL $(p<0.008))$ resulted in the best model for binary outcome in SCIM-MobS.

\section{URP-CTREE}

Figures 6.1a and $b$ and Figures 6.2 and 6.3 show the results of the URP-CTREE for the outcomes QtG, SCIM-SS and SCIM-MobS at 6 months after cervical SCI.

The URP-CTREE revealed that a combination of three significant single muscle predictor variables are sufficient to predict a range of different outcomes in QtG, SCIMSS and SCIM-MobS and identify homogenous subgroups from the cohort of cervical SCI individuals. For the QtG outcome, the URP-CTREE model with the QIG predictors (Figure 6.1b) showed similar results (medians and group formation) compared to the URP-CTREE model with the muscle predictor variables (Figure 6.1a).

As the interpretation of Figures $6.1 \mathrm{a}$ and $\mathrm{b}$ and Figures 6.2 and 6.3 are analogous, the more detailed interpretation of the URP-CTREE results will be limited to Figure 6.2 with the URP-CTREE for self-care at 6 months. The algorithm resulted in a partition of the initial cervical SCl cohort $(n=57)$ into 4 subgroups, which are represented as final nodes, based on three significant muscle predictor variables (bilateral) at one month. The final nodes represent subgroups with different outcomes ranging from low (least favorable) to high (most favorable) values for SCIM-SS at 6 months. 


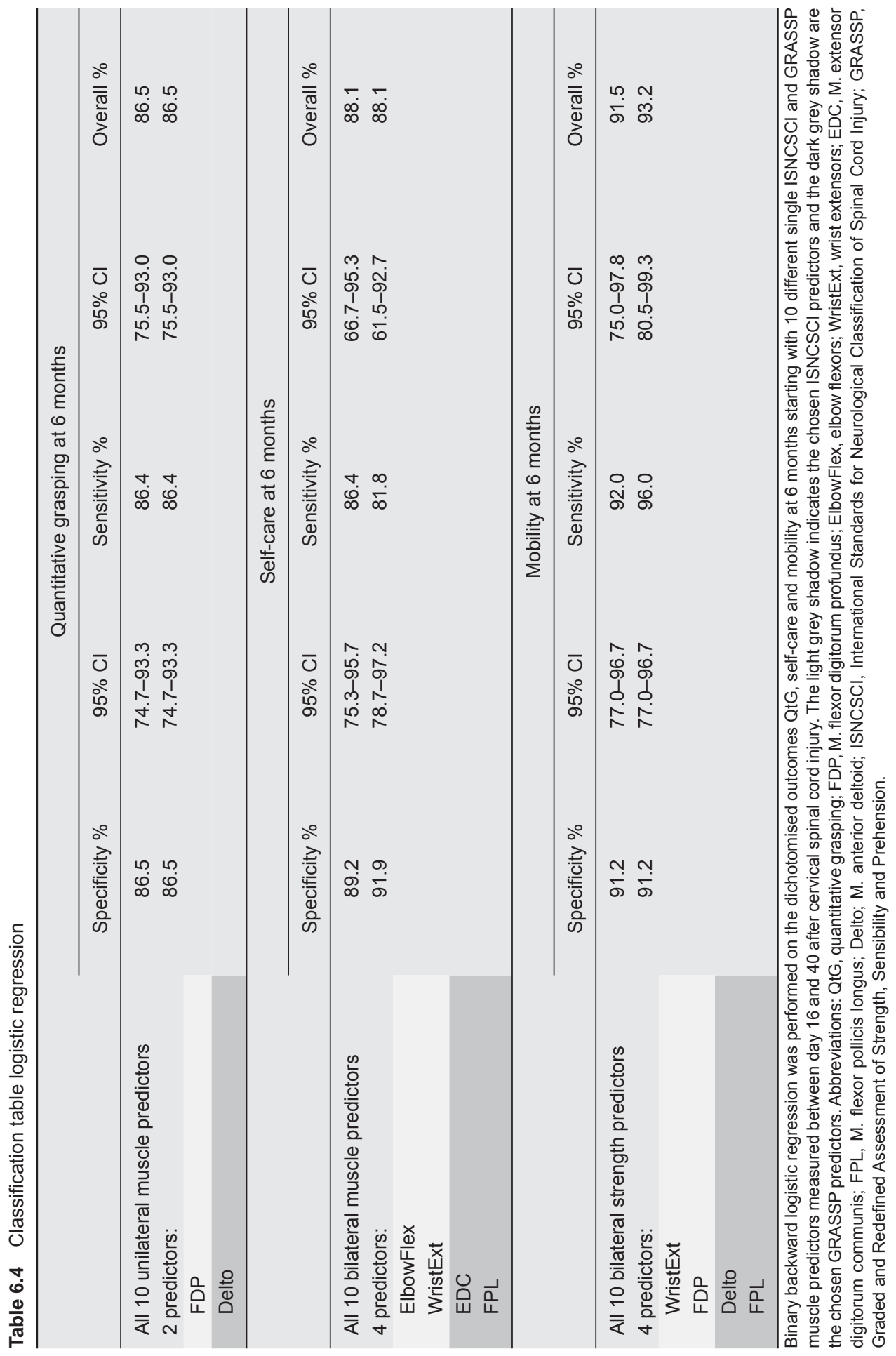



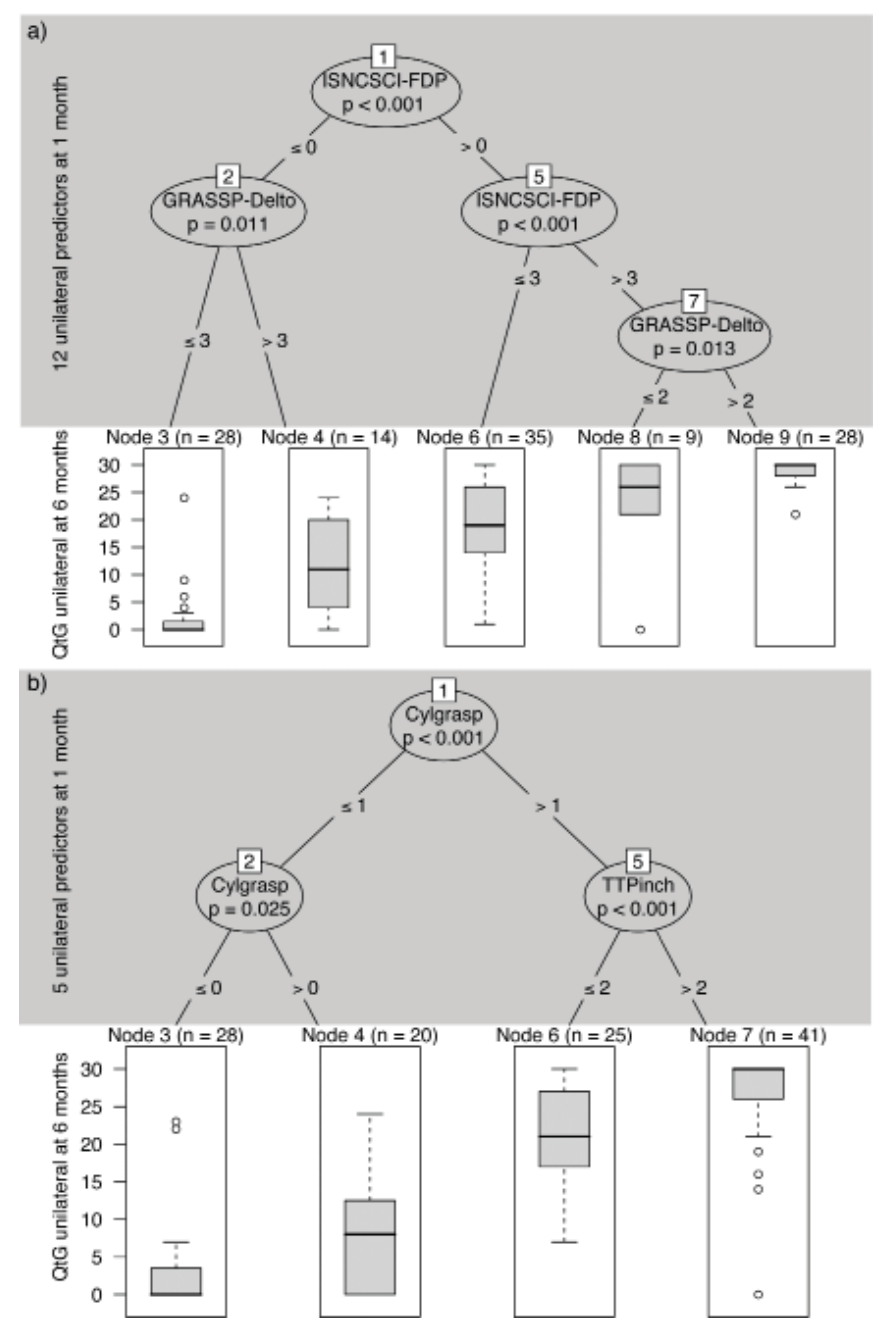

Figure 6.1 Unbiased recursive partitioning conditional inference tree (URP-CTREE) for quantitative grasping at 6 months starting with ten different single unilateral ISNCSCI and GRASSP muscle predictors and two unilateral ISNCSCI predictors AIS and MLI measured between day 16 and 40 after cervical spinal cord injury.

(a) The algorithm led to a partition of the initial cervical SCl cohort into five subgroups, which are represented as terminal nodes. Node size (number of arms) is indicated above each terminal node. From left to right, the terminal nodes represent subgroups from low (least favorable) to high (most favorable) quantitative grasping outcome at 6 months. The ISNCSCI-FDP was selected as the first unilateral predictor variable $(p<0.001)$ and split the cohort into two newly formed subgroups. The initial ISNCSCI-FDP cutoff values $\leq 0$ or $>0$ are indicated at the "branches". At each branch, a multiple-testing-adjusted p-value is given which describes the strength of the statistical association between predictor and outcome variable. Further separation is achieved by GRASSP-Delto for patients with $\leq 0$ ISNCSCI-FDP and by ISNCSCI-FDP and GRASSP-Opp for patients with > 0 ISNCSCI-FDP. (b) Unbiased recursive partitioning conditional inference tree (URP-CTREE) for quantitative grasping at 6 months starting with three single unilateral GRASSP-QIG predictors and two unilateral ISNCSCI predictors AIS and MLI measured between day 16 and 40 after cervical spinal cord injury. As the interpretation of "a" and "b" are analogous, we refer readers to the explanatory notes for "a" for more details.

Abbreviations: QtG, quantitative grasping; QIG, qualitative grasping; n, arms; p, significance level; ISNCSCI, International Standards for Neurological Classification of Spinal Cord Injury; GRASSP, Graded and Redefined Assessment of Strength, Sensibility and Prehension; FDP, M. flexor digitorum profundus; Delto; M. anterior deltoid; Opp, M. opponens pollicis; QualCyl, cylindrical grasp; TTpinch, tip to tip pinch. 




Figure 6.2 Unbiased recursive partitioning conditional inference tree (URP-CTREE) for self-care at 6 months starting with ten different single bilateral ISNCSCI and GRASSP muscle predictors and two bilateral ISNCSCI predictors AIS and MLI measured between day 16 and 40 after cervical spinal cord injury.

The algorithm led to a partition of the initial cervical SCl cohort into four subgroups, which are represented as terminal nodes. Node size (subgroups sample size) is indicated above each terminal node. From left to right, the terminal nodes represent subgroups from low (least favorable) to high (most favorable) self-care outcome at 6 months. The ISNCSCl-Triceps was selected as the first bilateral predictor variable $(p<0.001)$ and split the cohort into one newly formed subgroup. The initial ISNCSCI-Triceps cutoff values $\leq 1$ or $>1$ are indicated at the "branches". At each branch, a multiple-testing-adjusted $\mathrm{P}$-value is given which describes the strength of the statistical association between predictor and outcome variable. Below the ISNCSCI-Triceps cut-off $\leq 1$, no further separation is achieved and the next separation is achieved by GRASSP-DI1 and GRASSP-Delto for patients with > 1 ISNCSCI-Triceps.

Abbreviations: $n$, sample size; $p$, significance level; SCIM-SS, spinal cord independence measure: self-care subcategory; ISNCSCI, International Standards for Neurological Classification of Spinal Cord Injury; GRASSP, Graded and Redefined Assessment of Strength, Sensibility and Prehension; DI1, first dorsal interosseous; Delto; anterior deltoid; AIS, American Spinal Injury Association Impairment Scale; MLI; motor level of injury; C, cervical dermatome, T, thoracic dermatome. 


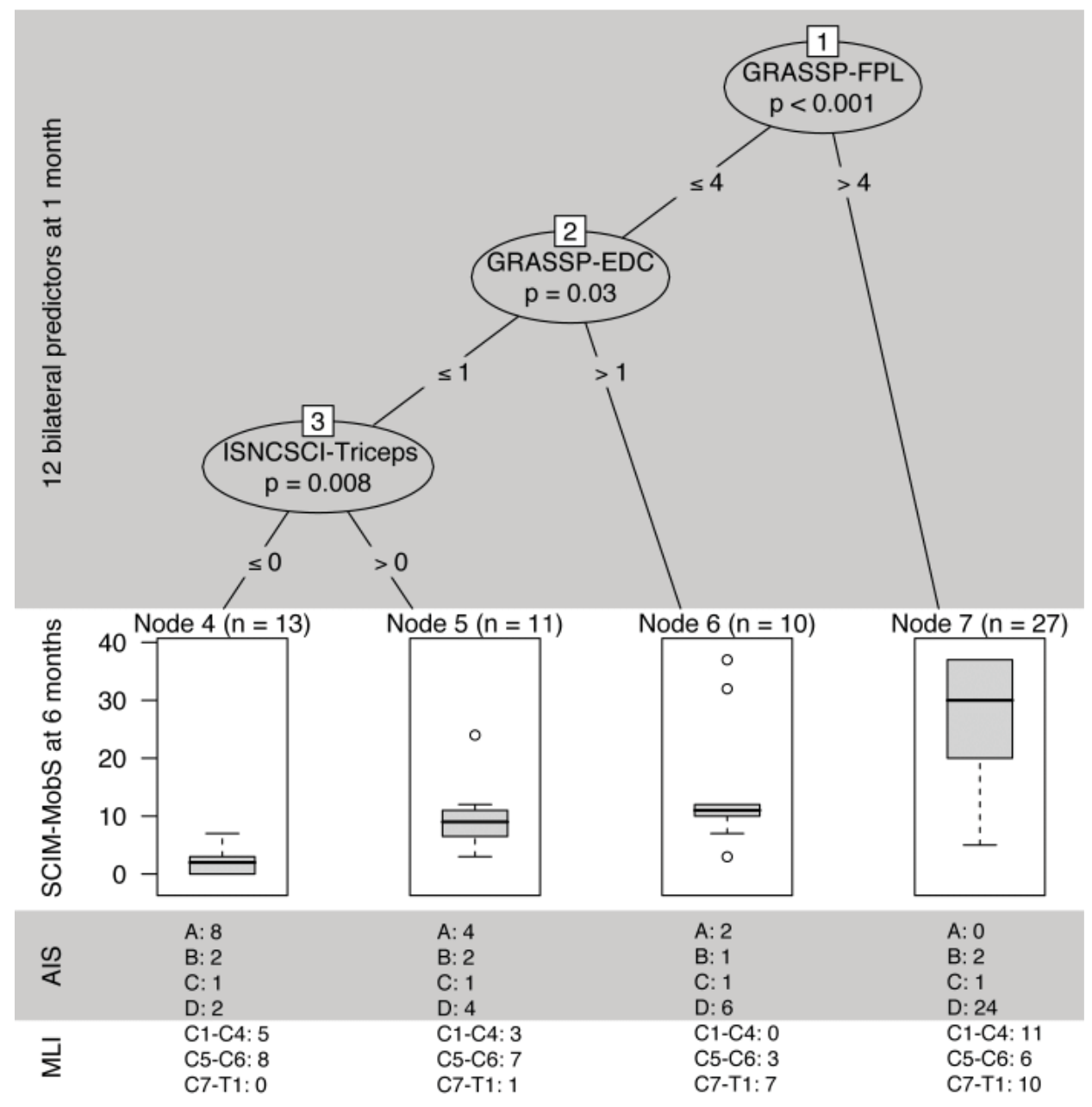

Figure 6.3 Unbiased recursive partitioning conditional inference tree (URP-CTREE) for mobility at 6 months starting with ten different single bilateral ISNCSCI and GRASSP muscle predictors and two bilateral ISNCSCI predictors AIS and MLI measured between day 16 and 40 after cervical spinal cord injury.

As the interpretation of Figure 6.2 and Figure 6.3 are analogous, we refer readers to the explanatory notes for Figure 6.2 for more details.

Abbreviations: $n$, sample size; p, significance level; SCIM-MobS, spinal cord independence measure: mobility subcategory; ISNCSCI, International Standards for Neurological Classification of Spinal Cord Injury; GRASSP, Graded and Redefined Assessment of Strength, Sensibility and Prehension; FPL, M. flexor pollicis longus; EDC, M. extensor digitorum communis, AIS, American Spinal Injury Association Impairment Scale; MLI; motor level of injury; C, cervical dermatome, $\mathrm{T}$, thoracic dermatome. 
The ISNCSCI-Triceps was selected as the first predictor variable $(p<0.001)$ and separates the population into two newly formed subgroups. The ISNCSCl-Triceps cutoff values $\leq 1$ or $>1$ are indicated at the "branches". At each branch, a multiple-testing-adjusted $p$-value is given which describes the strength of the statistical association between predictor and outcome variable. Below the ISNCSCI-Triceps cut-off $\leq 1$, no further separation was achieved (node $2: n=14$, least favorable outcome). Proceeding from the ISNCSCI-Triceps cut-off $>1$ subgroup $(n=47)$, the next separation was achieved with the DI1 cut-off at 6 points $(p=0.001)$. Proceeding from the GRASSP-DI1 cut-off $\leq 6$ subgroup $(n=36)$, the last separation was performed with the GRASSP-Delto $(p=0.017)$, identifying 2 subgroups with the GRASSP-Delto cut-off $\leq 7$ (node $5: n=17$ ) and GRASSP-Delto cut-off $>7$ (node 6: $n=19$ ) subgroups. Below the GRASSP-DI1 cut-off $>6$, no further subdividing was achieved (node 7: $\mathrm{n}=11$, most favorable outcome).

\section{Discussion}

The aims of this study were to investigate which single or combined upper limb muscles as defined by ISNCSCI-UEMS and GRASSP best predict upper limb function and independence in ADLs and to assess the predictive value of QIG on upper limb function in individuals with acute tetraplegia. The study reveals that the early assessment of motor strength of specific upper limb muscles is of high predictive value for the recovery of upper limb function and independence in ADLs at 6 months after cervical SCI. The combination of proximal and distal upper limb muscles as well as the early ability to initiate simplified grasp movements (i.e. CylGrasp, LatPinch and TTPinch), predicted upper limb function very well. Statistical methods not only allowed the elucidation of the distribution of outcomes following acute cervical SCI but also the prediction of specific cohorts of outcomes that may be specifically targeted for clinical intervention.

\section{Logistic regression}

Correlations between baseline predictors (i.e. upper limb strength) and functional outcomes following acute $\mathrm{SCl}$ have been shown in previous studies. ${ }^{7,12,29}$ Here, logistic regression was applied to reveal the best constellation of shared predictors on defined upper limb outcomes. Not unexpectly, a combination of standard ISNCSCI muscle groups i.e. FDP, ElbowFlex and WristExt, and additional GRASSP muscles, i.e. FPL, EDC and Delto, showed the best predictive value for the targeted outcomes QtG, SCIM-SS and SCIMMobS. Specifically, including a proximal shoulder muscle, in this case M. deltoid, improved 
prediction as this muscle contributes greatly to daily activities like transferring from bed, toilet or a car to the wheelchair, dressing the upper body or pouring water from a bottle into a cup. If the proximal shoulder and arm cannot be actively positioned and controlled as needed, the ability to use the hand for functional activities will be severely limited. Our refinement in including additional distal key muscles of the hand and fingers also contributed to improved prediction. Activities such as feeding, buttoning a shirt, inserting a key into a lock and turning it depend also on fine hand, finger and thumb movements, i.e. WristExt, FDP, EDC and to a major extend FPL. Our data are comparable to findings in a prior cross-sectional study on upper limb function in cervical $\mathrm{SCl},{ }^{24}$ in which a combination of distal muscle groups was proven especially useful in predicting self-care independence.

By performing a LR test, we demonstrated that the reduced model displays a greater predictive capacity than a logistic model containing all ten muscle predictors - an unexpected finding. A combination of a reduced number of proximal and distal muscles provided the same accuracy as the full model. This information is lost when using summed scores because in this case it is still unclear which single muscles contribute to outcome improvement. Moreover, in clinical settings and for research, the muscles to be tested should only include those of which that are useful in predicting functional outcome. The use of a combination of single predictors to enable more reliable prediction of long-term functional outcomes after $\mathrm{SCl}$ is in accordance with findings reported in previous longitudinal studies, ${ }^{7,14,16}$ although they have, in contrast, focused on total maximum summed scores combined with other predictor variables, making comparisons potentially unreliable.

\section{URP-CTREE}

Applying unbiased recursive partitioning resulted in the selection of a combination of proximal and distal muscles (i.e.Triceps and Delto, and FDP, EDC, DI1, FPL and Opp) as the best significant predictor variables for the outcomes QtG, SCIM-SS and SCIM-MobS. The advantage of URP-CTREE lies in the provision of a decision tree with specific threshold values (e.g. muscle strength scored from 0-5) for all outcomes. Our results demonstrated that a combination of a maximum of three significant muscle predictor variables are sufficient to predict a range of different outcomes (least favorable to most favorable) in QtG, SCIM-SS and SCIM-MobS and can reliably identify homogeneous subgroups from the cohort of cervical SCI individuals.

The AIS and MLI predictor variables were not chosen in the URP-CTREE model, indicating that AIS and MLI do not improve prediction accuracy. The combination ISNCSCI- 
UEMS and GRASSP muscle predictors with threshold values above the branches (URPCTREE) are therefore excellent indices for the stratification of patient groups and a good proxy for least and most favorable outcome irrespective of AIS and MLI. The AIS and motor level shown in the terminal nodes of Figure 6.2 and Figure 6.3 demonstrate the high heterogeneity across all nodes at 6 months.

The URP-CTREE finding that GRASSP-QIG items predicted upper limb function accurately is exciting because QIG can be quickly and easily assessed at the bedside in almost all clinical settings and highlights the importance of including this relative simply measure in standard clinical evaluations.

\section{Clinical and research implications}

The high variability in neurological recovery requires reliable and sensitive prognostic tools for cervical SCl outcomes if we are to improve the design and conduct of clinical trials. This includes the stratification and enrollment of the most suitable patients (e.g. avoiding the enrollment of patients who will recover well irrespective of treatment) and to provide information regarding meaningful clinical outcome thresholds. Simplified, visually informative yet sensitive prediction models like URP-CTREE are of great value in the clinical trial setting. The magnitude of recovery and expected functional outcome is also of great importance for future rehabilitation interventions and to provide early prognostic information to patients and their families.

Most interestingly, the testing of qualitative grasping (QIG) may be a promising assessment tool as it can be applied easily in individuals with acute tetraplegia. QIG requires little time and can complement the standard assessment of muscle strength.

\section{Limitations}

The findings presented here are based on assessments at 1 month (range $=16-40$ days) after injury and cannot be easily applied to studies performed within the first days following $\mathrm{SCl}$. The effect of different time intervals on the prediction models following SCI needs to be evaluated in more detail. Validation of an independent data set (i.e. external validation) will also be required to prove to what extent our findings can be generalised.

\section{Conclusion}

Our data show that prediction of upper limb function and independence in ADLs at 6 months can be accurately achieved through a combination of a limited number of single 
proximal and distal muscle strength tests as provided by the ISNCSCI-UEMS and GRASSP standards in individuals with acute cervical SCI. Furthermore, the combination of ISNCSCI-UEMS and GRASSP muscle predictors are ideal indices for stratifying patient groups and a good proxy for favorable and unfavorable outcomes irrespective of AIS and MLI. Qualitative grasping, a simple test with minimal time demands, predicted upper limb function very well. In summary, the predictive value of standard ISNCSCI-UEMS for upper limb function can be significantly improved with the addition of single GRASSP predictors.

\section{Acknowledgments}

We are grateful to the individuals with $\mathrm{SCI}$ who participated in this study and the cooperating centres, especially to those occupational therapists who recruited and tested the participants: Spinal Cord Injury Center, Hohe Warte, Bayreuth, Germany, Orthopädische Universitätsklinik Heidelberg, Germany, Unfallklinik Murnau, Germany, Balgrist University Hospital, Zurich, Switzerland, and the Swiss Paraplegic Center Nottwil, Switzerland. Furthermore, we thank Dr Aurelio Tobon (Nottwil) for performing the clinical neurological examinations and Mr Tim Killeen (Balgrist) for reviewing the article and his invaluable assistance with English language editing.

\section{Funding}

The authors disclose receipt of the following financial support for the research, authorship, and/or publication of this article: The study was supported by the Swiss Paraplegic Center, Nottwil, Switzerland, and funded by the Clinical Research Priority Program NeuroRehab of the University of Zurich, Switzerland.

\section{References}

1. Kirshblum SC, Waring W, Biering-Sorensen F, et al. Reference for the 2011 revision of the International Standards for Neurological Classification of Spinal Cord Injury. J Spinal Cord Med 2011;34:547-554.

2. Steeves JD, Kramer JK, Fawcett JW, et al. Extent of spontaneous motor recovery after traumatic cervical sensorimotor complete spinal cord injury. Spinal Cord 2011;49:257-265.

3. Marino RJ, Burns S, Graves DE, Leiby BE, Kirshblum S, Lammertse DP. Upper- and lowerextremity motor recovery after traumatic cervical spinal cord injury: an update from the national spinal cord injury database. Arch Phys Med Rehabil 2011;92:369-375.

4. Kramer JL, Lammertse DP, Schubert M, Curt A, Steeves JD. Relationship between motor recovery and independence after sensorimotor-complete cervical spinal cord injury. Neurorehabil Neural Repair 2012;26:1064-1071. 
5. Ditunno JF, Jr., Cohen ME, Hauck WW, Jackson AB, Sipski ML. Recovery of upper-extremity strength in complete and incomplete tetraplegia: a multicenter study. Arch Phys Med Rehabil 2000;81:389-393.

6. Velstra IM, Curt A, Frotzler A, et al. Changes in Strength, Sensation, and Prehension in Acute Cervical Spinal Cord Injury: European Multicenter Responsiveness Study of the GRASSP. Neurorehabil Neural Repair 2015.

7. Velstra IM, Bolliger M, Tanadini LG, et al. Prediction and Stratification of Upper Limb Function and Self-Care in Acute Cervical Spinal Cord Injury With the Graded Redefined Assessment of Strength, Sensibility, and Prehension (GRASSP). Neurorehabil Neural Repair 2014;28:632642.

8. Steeves JD, Lammertse DP, Kramer JL, et al. Outcome Measures for Acute/Subacute Cervical Sensorimotor Complete (AIS-A) Spinal Cord Injury During a Phase 2 Clinical Trial. Top Spinal Cord Inj Rehabil 2012;18:1-14.

9. Kirshblum SC, Biering-Sorensen F, Betz R, et al. International Standards for Neurological Classification of Spinal Cord Injury: cases with classification challenges. J Spinal Cord Med 2014;37:120-127.

10. Kalsi-Ryan S, Curt A, Fehlings MG, Verrier MC. Assessment of the Hand in Tetraplegia Using the Graded Redefined Assessment of Strength, Sensibility and Prehension (GRASSP): Impairment Versus Function. Top Spinal Cord Inj Rehabil 2009;14:34-46.

11. Velstra IM, Bolliger M, Baumberger M, Rietman JS, Curt A. Epicritic sensation in cervical spinal cord injury: diagnostic gains beyond testing light touch. J Neurotrauma 2013;30:1342-1348.

12. Kalsi-Ryan S, Beaton D, Curt A, et al. The Graded Redefined Assessment of Strength Sensibility and Prehension: reliability and validity. J Neurotrauma 2012;29:905-914.

13. Curt A, Dietz V. Traumatic cervical spinal cord injury: relation between somatosensory evoked potentials, neurological deficit, and hand function. Arch Phys Med Rehabil 1996;77:48-53.

14. van Middendorp JJ, Hosman AJ, Donders AR, et al. A clinical prediction rule for ambulation outcomes after traumatic spinal cord injury: a longitudinal cohort study. Lancet 2011;377:10041010.

15. Wilson JR, Grossman RG, Frankowski RF, et al. A clinical prediction model for long-term functional outcome after traumatic spinal cord injury based on acute clinical and imaging factors. J Neurotrauma 2012;29:2263-2271.

16. Zorner B, Blanckenhorn WU, Dietz V, Curt A. Clinical algorithm for improved prediction of ambulation and patient stratification after incomplete spinal cord injury. J Neurotrauma 2010;27:241-252.

17. Kuhn F, Halder P, Spiess MR, Schubert M. One-year evolution of ulnar somatosensory potentials after trauma in 365 tetraplegic patients: early prediction of potential upper limb function. $\mathrm{J}$ Neurotrauma 2012;29:1829-1837.

18. Kalsi-Ryan S, Beaton D, Curt A, Popovic MR, Verrier MC, Fehlings MG. Outcome of the upper limb in cervical spinal cord injury: Profiles of recovery and insights for clinical studies. J Spinal Cord Med 2014;37:503-510.

19. Catz A, Itzkovich M, Agranov E, Ring H, Tamir A. SCIM--spinal cord independence measure: a new disability scale for patients with spinal cord lesions. Spinal Cord 1997;35:850-856. 
20. Itzkovich M, Gelernter I, Biering-Sorensen F, et al. The Spinal Cord Independence Measure (SCIM) version III: reliability and validity in a multi-center international study. Disabil Rehabil 2007;29:1926-1933.

21. Catz A, Itzkovich M, Tesio L, et al. A multicenter international study on the Spinal Cord Independence Measure, version III: Rasch psychometric validation. Spinal Cord 2007;45:275291.

22. Anderson KD, Acuff ME, Arp BG, et al. United States (US) multi-center study to assess the validity and reliability of the Spinal Cord Independence Measure (SCIM III). Spinal Cord 2011;49:880-885.

23. Schuld C, Wiese J, Hug A, et al. Computer implementation of the international standards for neurological classification of spinal cord injury for consistent and efficient derivation of its subscores including handling of data from not testable segments. J Neurotrauma 2012;29:453461.

24. Rudhe C, van Hedel HJ. Upper extremity function in persons with tetraplegia: relationships between strength, capacity, and the spinal cord independence measure. Neurorehabil Neural Repair 2009;23:413-421.

25. Hothorn T, Hornik K, Zeileis A. Unbiased recursive partitioning: a conditional inference framework. J Comput Graph Stat 2006;15:651-674.

26. Tanadini LG, Steeves JD, Hothorn T, et al. Identifying Homogeneous Subgroups in Neurological Disorders: Unbiased Recursive Partitioning in Cervical Complete Spinal Cord Injury. Neurorehabil Neural Repair 2014;28:507-515.

27. Team. RDC. R: A language and environment for statistical computing. [online]. Available at: http://www.Rproject.org/.

28. Hothorn T, Hornik K, Strobl C, al. e. party: A Laboratory for Recursive Partitioning [online]. Available at: http://cran.r-project.org/web/packages/party/index.html.

29. Curt A, Keck ME, Dietz V. Functional outcome following spinal cord injury: significance of motor-evoked potentials and ASIA scores. Arch Phys Med Rehabil 1998;79:81-86. 

Chapter 7

General discussion 
The aim of this final chapter is to accumulate the findings from the previous chapters and to present clinical implications. In addition, methodological considerations and areas for future research will be formulated.

After cervical spinal cord injury (SCI), motor and sensory impairments cause limitations in upper limb function, which affect the performance of activities of daily living (ADLs), independence and, ultimately, restrict participation and quality of life. Previous studies have shown that individuals with tetraplegia consider improvements in upper limb function to be one of the most significant factors in improving quality of life..$^{1-3}$ However, appropriate outcome measures to evaluate the efficacy of rehabilitation and experimental interventions are lacking and research in this field is limited. ${ }^{4-8}$ Furthermore, cervical $\mathrm{SCl}$ shows large variability, ${ }^{9,10}$ which makes interpretation of clinical findings and research difficult. Therefore, the overall objective of this $\mathrm{PhD}$ thesis has been to study the assessment, evaluation and prediction of upper limb function up to one year post injury using the Graded Redefined Assessment of Strength, Sensibility and Prehension (GRASSP) in individuals with cervical $\mathrm{SCl}$. In order to answer the research questions of this thesis, one systematic review, one multi-center cross-sectional study and one multi-center longitudinal cohort study was performed which is presented in the five chapters of this thesis. Evidence was presented that the GRASSP provides information on multifaceted domains (e.g. strength, sensation and prehension) and allows to detect both subtle and clinical meaningful changes in upper limb function. Furthermore, the GRASSP can accurately predict upper limb function and ADLs, even in a heterogeneous group of individuals across a wide spectrum of neurological recovery. This supports the use of the GRASSP in the assessment of rehabilitation outcome as well as in clinical studies and trials.

\section{Upper limb function and clinical implications}

A systematic review of outcome measures for upper extremity function (chapter 2) was performed and these were classified on several levels according to the International Classification of Functioning, Disability, and Health (ICF). ${ }^{11}$ In the systematic review of the available outcome measures, the five most frequently used outcome measures in the four study populations were determined: (1) peripheral upper extremity conditions, (2) rheumatologic diseases, (3) stroke, and (4) tetraplegia, which resulted in a total of 17 outcome measures. The outcome measures show large variability with regard to the areas of functioning and disability addressed. It appears that there clearly is a gap in the availability of upper extremity-specific outcome measures in studies of individuals with tetraplegia. 
Information on psychometric properties of outcome measures is lacking for this specific population. This deficiency is a hindrance for rehabilitation and research in cervical $\mathrm{SCl}$, because relevant outcome measures are not validated and consequently not used. For example, the Disability of the Shoulder, Arm and Hand Questionnaire (DASH) ${ }^{12}$ has still not been validated for tetraplegia, even though it could provide great insight into activity limitations and participation restrictions of individuals with tetraplegia. Further research should be performed to study the psychometric properties of the DASH in cervical SCI. The results of the systematic review help clinicians and researchers to select the most appropriate outcome measure for their clinical population or research question according to ICF-based content validity, and psychometric properties of the measures.

The lack of relevant outcome measures to investigate impairment changes over time and how they contribute to complex upper limb tasks, was the rationale and starting point for the development of the Graded and Redefined Assessment of Strength, Sensibility and Prehension (GRASSP). ${ }^{13}$ It is an outcome measure that quantifies impairment as well as functional outcome, and it will help clinicians to adjust rehabilitation measures and treatment strategies in order to achieve better outcomes. In individuals with chronic SCI (i.e. more than 6 months post-injury), the validity and reliability of GRASSP were found to be good. ${ }^{14}$ The responsiveness of the GRASSP (chapter 3 ) was defined as its sensitivity in detecting changes, ${ }^{15-18}$ making it possible to evaluate recovery patterns and treatment outcomes in cervical SCI. It was found that the GRASSP has excellent responsiveness for the evaluation of upper limb outcomes up to one year after cervical SCI. ${ }^{19}$ The observed GRASSP subtest changes were also in accordance with the clinicians' impressions of patient changes, which is a novel finding. This finding suggests that the GRASSP is a clinically meaningful tool. Therefore, the clinician-rated outcome measure (CROM) may be useful in clinical trials for evaluating the progress of a patient. ${ }^{20}$ Experienced clinicians, such as these involved in this study, have a good understanding of neurological impairment and functional performance.

The GRASSP detected clinically significant changes complimentary to the International Standards of Neurological Classification of Spinal Cord Injury (ISNCSCI) and Spinal Cord Independence Measure self-care subscale (SCIM-SS). The combined assessment of neurological (body structure and body function) and functional outcomes, for example, prehension (activity and participation), focused on segmental cervical spinal cord functions that are closely related to other standard assessment tools (ISNCSCI and SCIM). This combination supports the use of GRASSP in the assessment of rehabilitation as well as in interventional clinical trials, which seek to detect both subtle and clinical meaningful changes. 
Furthermore, in chapter 3 more detailed strength and prehension recovery profiles are presented ${ }^{19}$ compared to most other published reports. ${ }^{9,10,21-27}$ In the past, the understanding of recovery in cervical SCI was mainly based on ISNCSCI subcategories like the upper extremity motor score (UEMS) and ASIA impairment scale (AIS) grade.9,23-26,28,29 It was the overall aim of the GRASSP development to provide a measurement tool of upper limb outcome/function on different ICF levels that will be more sensitive and more comprehensive regarding the neurological changes compared to other measures (e.g. ISNCSCI, SCIM). The data suggest that this has been achieved, and comparable studies in this field are yet to be done to confirm this finding.

The ISNCSCI is considered the gold standard in SCI, and is a measure of impairment that generates detailed scoring of segmental sensorimotor deficits. It has been proven to be of value in the diagnosis and prognosis of $\mathrm{SCl}$. It is designed as a classification measure to capture neurological deficits for the whole range of $\mathrm{SCl}$, making it non-specific to the upper limb. Chapter 3 provides evidence that the GRASSP is more responsive than ISNCSCI. ${ }^{19}$ The likely reasons for the superior responsiveness of the GRASSP muscle strength and sensation are the expanded combination of muscles and the broader scaling of sensation (by applying different sensory modalities) as well as the additional palmar test locations, respectively.

Furthermore throughout this thesis, the SCIM III was used as a global outcome measure to establish clinically meaningful categorization of functioning in ADLs. In the past ${ }^{30}$ as well as in chapter 3 , the SCIM-SS has been found to be responsive to change. ${ }^{19}$ Likewise it does not provide insights into the underlying sensorimotor function driving functional recovery. Accordingly, the SCIM is not positioned to discern functional improvement arising from actual repair or damaged spinal cord tissue versus rehabilitation training, motivation and mood factors when performing tasks. The SCIM's focus is on gained independence and thus cannot differentiate whether the tasks are performed bimanually or with compensatory movements. ${ }^{30}$ Therefore, relying on an activity measure like the SCIM introduces the risk of other unaccounted factors contributing to the outcome and altering the accurate interpretation of a benefit realized by a therapeutic intervention.

Several other assessments such as the Tetraplegie Hand Activity Measure (THAQ), ${ }^{31}$ the Van Lieshout Test (VLT), ${ }^{32,33}$ the Motor Capacity Scale (MCS), ${ }^{34,35}$ Capability of Upper Extremity Test (CUE) ${ }^{36,37}$ and Grasp Release Test (GRT) ${ }^{38}$ have been developed, however these assessments are not designed to provide detailed and reliable information about the changes in specific sensory and motor impairments affecting upper limb function. 
The GRASSP subtest scores were able to disentangle motor and sensory functions contributing to the outcome of upper limb function. The GRASSP assessment reveals, how changes in function are related to neurological improvements following $\mathrm{SCl}$. In addition, the GRASSP subtests also include standardised prehension tests that are related to changes in neurological outcomes. These combined assessments permit to determine whether changes in function are based on improvement through compensatory movements or on improvement of neurological function. Accordingly, quantitative grasping (QtG) provides a detailed scoring of standardised tasks focusing on the grasp form and is therefore able to explain how changes up to 1 year post injury are achieved.

\section{Sensation and clinical implications}

Light touch (LT) and pinprick (PP) assessments examined according to the ISNCSCI ${ }^{39}$ are used routinely during neurological examination of sensibility in $\mathrm{SCl}$ patients. Research is impeded by limited test reliability, which is true for all sensory qualities, such as epicritic sensation and prothopatic sensation, to some extent. ${ }^{40-44}$ The assessment of different levels of sensory impairment is furthermore limited, because the subjective rating of patients is not able to define incremental levels of impairment, but represents a rather simplified categorical (ordinal scale, e.g., normal, impaired, and abolished sensation) gross scoring. Therefore, it is very difficult to determine minor changes during recovery (improvements or deterioration), and the clinical testing of one specific sensory quality, such as LT, within a complex domain of sensory function, such as epicritic sensation, conveyed by dorsal column pathways will likely have limited sensitivity. The Semmes and Weinstein monofilaments (SWM) and the electrical perception threshold (EPT) contain a greater range of discernible response levels for detecting a tactile cutaneous sensation and electrical stimulation, respectively. Thus they have the potential of being more sensitive. Chapter 4 is the first report on the degree of agreement between the two complementary quantitative methods (SWM or EPT) and the ISNCSCI-LT. It has been shown that SWM or EPT reveal deficits that LT alone does not. Therefore, this study provides evidence that the segmental assessment of epicritic sensation is improved by SWM or EPT. This is important in early clinical trials (phase 1, phase 2) where segmental and subtle changes in sensory function provide important information about the beneficial or detrimental (i.e. descending or ascending levels of lesion) effects of a novel intervention. The EPT or SWM should be used in combination with the clinical sensory examination in order to improve the sensitivity to discrete sensory changes and the robustness of sensation examination in 
clinical practice and research. Furthermore, comparisons between individual dermatomes can be performed. Therefore the chapters 3 and 4 of this thesis are timely in that they extend the quest for improved assessment techniques that reveal the extent of SCI during the first $6 / 12$ months. This time window is relevant for clinical trials of rehabilitative or regenerative treatments for the restoration of function in $\mathrm{SCl}$ individuals. As such, these papers will attract the attention of those designing clinical trials for the recovery of $\mathrm{SCl}$ and will influence the selection of outcome measures.

\section{Prediction of upper limb function and clinical implications}

Reliable prediction of functional outcomes can be used to formulate rehabilitation goals and regimens and is essential for improving the stratification of patients for clinical interventions, for which the enrollment of rather homogenous patient cohorts is required. Improved stratification rules will be of benefit in trials assessing the safety and efficacy of interventions, where the detection of even subtle changes is crucially important in the evaluation of therapies. In chapter $\mathbf{5}$, the focus was on prediction and stratification of upper limb function and self-care after cervical SCI. Results indicate that the GRASSP at 1 month can predict functional outcome at 6 and 12 months accurately, even in a heterogeneous group of individuals across a wide spectrum of neurological recovery. Moreover, evidence has been found that the GRASSP motor scoring (manual muscle testing (MMT) in particular) has an excellent predictive value for clinical outcomes at 6 and 12 months after cervical $\mathrm{SCl}$ with a high sensitivity and specificity. Both receiver operating characteristics (ROC) and logistic regression analyses corroborate the high within-sample validity of the MMT within GRASSP as a predictor variable. Semmes and Weinstein monofilament although less influential than muscle strength, is also able to predict outcome of self-care and upper limb function and is specifically useful for prediction when motor assessments are limited (for instance when key motor muscles are not defined above C5) or when muscle activation is hindered by other factors, such as limb fractures or bruising. Logistic regression and ROC analysis do not, however, provide sufficient information about the distribution of outcomes. For this reason, also a regression tool from the family of unbiased recursive partitioning methods called conditional inference tree ${ }^{45}$ (URP-CTREE) was used. Due to the relatively low incidence of traumatic $\mathrm{SCl}^{46}$ the recruitment of individuals for clinical studies is limited. Cervical $\mathrm{SCI}$ comprises a heterogeneous population regarding the diversity of recovery patterns as well as the severity and level of injury, ${ }^{47}$ which results in narrow inclusion criteria. The consequences are slow enrollment rates and prolonged study 
duration. Conditional inference trees ${ }^{45}$ overcome some of these challenges. The results in chapter $4^{48}$ show that simplified, visual informative models like URP-CTREE enable the prediction of the distribution of different outcomes in acute cervical $\mathrm{SCl}$ and the definition of more homogenous outcome cohorts for the stratification of study participants, which has also been shown in other recent studies. ${ }^{28,45}$ Within the URP-CTREE analysis, in line with logistic regression and ROC analyses, MMT remained the strongest predictor for upper limb function and self-care outcome. The motor scoring, as defined in the GRASSP, includes a greater number of muscles compared to the UEMS within ISNCSCI by incorporating distal and proximal muscle groups. This expanded combination of muscle groups probably contributes to the high outcome prediction seen in this study, lending further support to use the GRASSP as a standardised assessment tool of upper limb function. Studies that assess the significance of combining individual parameters to improve outcome prediction are sparse. ${ }^{49,50}$ Based on the results in chapter 5 , the combination of MMT with other predictors, such as qualitative grasping (Q|G) and quantitative grasping (QtG), can improve outcome prediction.

At the beginning of this thesis, little knowledge was available on the prediction of functional outcome in $\mathrm{SCl}$. In recent years, there have been several investigations into the prediction of functional outcome after $\mathrm{SCl},{ }^{49-52}$ increasing our knowledge of recovery and prediction of upper limb function and self-care after cervical SCI. ${ }^{19,48,53,54}$ Evidence was presented that the GRASSP allows the precise identification of recovery profiles ${ }^{19}$ and accurately predicts upper limb function and self-care in acute tetraplegia. ${ }^{48}$ However, there is still limited evidence regarding the influence of each individual muscle or muscle group on the prediction of upper limb function and ADLs. Furthermore, the effect of specific grasp patterns, as described in the GRASSP, on the prediction of upper limb function and functional outcome has not yet been investigated. Therefore, chapter 6, describes the predictive value of upper limb muscles and grasp patterns for functional outcome in cervical $\mathrm{SCl}$. The UEMS is often used in clinical research to examine the course of spontaneous

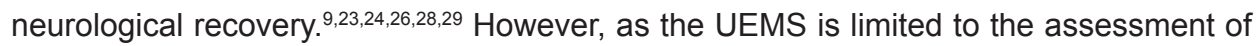
only five key muscle groups for each upper limb in cervical SCI and individuals with cervical $\mathrm{SCl}$ can show a high variability in motor recovery following acute injury, the precision of the UEMS regarding motor recovery is not sufficient. The GRASSP-MMT assesses ten muscles, of which five are also measured in ISNCSCI-UEMS. The number of muscles to be tested should be kept to a minimum and should only include muscles that are as useful in the clinical setting and for research. The data in chapter 6 show that prediction of upper 
limb function and independence in ADLs at 6 months can be accurately achieved using a combination of some single proximal and distal muscle strength tests, as provided by the ISNCSCI-UEMS, and the GRASSP-MMT in individuals with acute cervical SCI. The AIS and motor level of injury (MLI) predictor variables were not chosen in the URP-CTREE model, indicating that AIS and MLI do not improve prediction accuracy. Therefore, AIS grades and MLI might not be appropriate for stratification in clinical studies or trials. Furthermore, the combination of these limited muscle predictors are ideal indices for stratifying patient groups and a good proxy for favorable and unfavorable outcomes irrespective of AIS and MLI.

In addition, the URP-CTREE finding that GRASSP-QIG items predict upper limb function accurately is exciting, because QIG can be assessed quickly and easily at the bedside in almost all clinical settings. This highlights the importance of including this relative simply measure in standard clinical evaluations. Therefore, the predictive value of standard ISNCSCI-UEMS for upper limb function can be improved significantly with the addition of single GRASSP predictor. From a clinical point of view, it is therefore worthwhile to consider the redundancies and benefits of the different motor testing methods used in the clinic, in order to optimise time resources.

\section{Methodological issues and future directions}

- $\quad$ As outlined within this thesis, cervical SCI can affect people in a number of different ways. Relatively narrow inclusion criteria have been used in these investigations. Individuals with high cervical lesions and continuous complete ventilator dependency and complete loss of upper limb control were excluded. Therefore, the results of this thesis are most appropriately generalised to individuals with at least a motor grade of 1 in the M. biceps bilaterally.

- $\quad$ A range of 61 to 74 participants were included in the longitudinal studies. Although this is not a large group, it can be considered to be a good representation of the total population with cervical SCI in Germany and Switzerland. Especially in the field of cervical SCl where the number of patients capable- and willing to participate in clinical research is relatively small and difficult to recruit. Multi-center studies are a solution for the low availability of study participants.

- $\quad$ Further investigations using an independent dataset will be required to prove to what extent the findings in this thesis can be generalised. It is recommended to set up similar longitudinal studies in other countries, in order to compare the results of our German / Swiss longitudinal study with those from other countries. 
- Both individuals with traumatic and non-traumatic tetraplegia were included. The overall findings have not been affected by including a few individuals with nontraumatic cervical SCl as the overall percentage of these was around 5 to $8 \%$ in the different studies. However, it would be of great interest to investigate the GRASSP in a non-traumatic SCl cohort. There is already an ongoing longitudinal study with individuals suffering from cervical spondylotic myelopathy in North America.

- $\quad$ In the longitudinal studies, the baseline measurement (within 10 days after $\mathrm{SCl}$ ) was excluded, due to the small sample size $(n=40)$. The effect of this early time interval is unknown and needs to be evaluated in more detail in longitudinal studies starting early after SCl.

- $\quad$ Novel questionnaires like the CROM have to be interpreted with caution as they may be influenced by other factors (e.g. clinical judgment, past experience, beliefs regarding treatment effectiveness etc.). Systematic bias in the results cannot be entirely excluded as some assessments of GRASSP and CROM in individual patients have been performed by the same therapist. Depending on the study design and research question, it is of course advisable that independent clinicians perform the GRASSP and CROM, thereby minimizing examiner bias.

- $\quad$ The URP-CTREE is applicable to several types of regression problems, including nominal, ordinal and numeric variables. ${ }^{45}$ Many clinical assessments like ISNCSCI, SCIM, and GRASSP were analysed as sum scores of different items and treated as continuous variables, even though they are ordinal scales. It is acknowledged that this could produce misleading results ${ }^{55}$ when summed scores rely on counts of potentially unequal units and do not represent a consistent metric scoring. The number of statistical methods to analyse this sort of data is limited and using Raschanalysis, ${ }^{56,57}$ e.g., should be considered in future studies.

- In clinical practice, the findings of this thesis will help to improve early management decisions, like discharge and multidisciplinary intervention planning at acute $\mathrm{SCl}$ rehabilitation centers. As a consequence, subsequent multidisciplinary rehabilitation services can be optimised in line with the probability for regaining some upper limb function and self-care independence. Knowing the underlying neurological changes of upper limb function, future research should be conducted to further understand the determinants of upper limb function. It would be of great interest to investigate, if and how factors (determinants) like specific injury causes, hand dominance preand post-injury, age, length of stay, standard therapies, rehabilitation programs or 
intervention frequency, duration of therapy sessions and the start of upper limb rehabilitation program affect upper limb function outcome and the validity of our results. Therefore, future large multi-center studies are needed to study the effect of different determinants, which play a role in outcome effectiveness studies, on upper limb function and thus the prescription of therapeutic interventions. It would also be interesting to determine the optimal time frame and type of intervention as well as to systematic collect and assess costs associated with an intervention (cost analysis).

Furthermore, the change scores up to one year warrant further investigation for their value in determining clinical endpoints for interventional studies, particularly those engaging individuals with acute traumatic cervical SCI.

There is little evidence supporting conventional therapies like splinting or developing a tenodesis grasp. ${ }^{58,59}$ In contrast, interventions targeting upper limb recovery and upper limb function are an area which is growing continually. Although efficacy of functional electrical stimulation (FES) ${ }^{60-62}$ and somatosensory stimulation ${ }^{63,64}$ has been established and despite the functional gains after upper limb surgery, ${ }^{65-70}$ as well as recent insights into an upper limb surgery registry, ${ }^{71}$ more well designed effectiveness studies are required. The measurement approaches must be standardised across centers and should have consistent baseline and follow up data to enable comparisons between studies and to assess efficacy. The comparison of intervention outcomes requires large homogeneous samples which will require cooperation between multiple centers around the world. GRASSP can serve as an initial step in elucidating upper limb recovery in tetraplegia and will help to establish outcomes that are useful for future clinical studies and trials.

\section{Conclusions}

a. This study provides clinicians and researchers with a guide for the selection of the most appropriate outcome measure for their clinical population or research question, taking ICF based content validity ("what do the outcome measures address?"), reliability and responsiveness into consideration.

b. The GRASSP showed excellent responsiveness within the first year after cervical $\mathrm{SCl}$. It detected distinct changes in strength and prehension relating to the severity of cervical SCI. GRASSP detected clinically significant changes complimentary to the ISNCSCI and SCIM-SS assessments. 
c. The additional measurements of epicritic sensation by SWM or EPT increased sensitivity by detecting and quantifying differences in sensory thresholds above, at and below the LT level of injury.

d. The GRASSP at 1 month accurately predicted upper limb function and selfcare outcomes at 6 and 12 months after cervical SCI. URP-CTREE revealed the distribution of outcome categories and can be used to predict cohorts with homogenous outcomes.

e. The predictive value of standard ISNCSCI-UEMS for upper limb function can be improved significantly with the addition of single GRASSP predictors.

\section{References}

1. Anderson KD. Targeting recovery: priorities of the spinal cord-injured population. J Neurotrauma 2004;21:1371-1383.

2. Hanson RW, Franklin MR. Sexual loss in relation to other functional losses for spinal cord injured males. Arch Phys Med Rehabil 1976;57:291-293.

3. Snoek GJ, MJ IJ, Hermens HJ, Maxwell D, Biering-Sorensen F. Survey of the needs of patients with spinal cord injury: impact and priority for improvement in hand function in tetraplegics. Spinal Cord 2004;42:526-532.

4. Alexander MS, Anderson KD, Biering-Sorensen F, et al. Outcome measures in spinal cord injury: recent assessments and recommendations for future directions. Spinal Cord 2009;47:582-591.

5. Dunn JA, Sinnott KA, Bryden AM, Connolly SJ, Rothwell AG. Measurement issues related to upper limb interventions in persons who have tetraplegia. Hand Clin 2008;24:161-168.

6. Mulcahey MJ, Hutchinson D, Kozin S. Assessment of upper limb in tetraplegia: considerations in evaluation and outcomes research. J Rehabil Res Dev 2007;44:91-102.

7. van Tuijl JH, Janssen-Potten YJ, Seelen HA. Evaluation of upper extremity motor function tests in tetraplegics. Spinal Cord 2002;40:51-64.

8. Velstra IM, Ballert CS, Cieza A. A systematic literature review of outcome measures for upper extremity function using the international classification of functioning, disability, and health as reference. PM R 2011;3:846-860.

9. Kramer JL, Lammertse DP, Schubert M, Curt A, Steeves JD. Relationship between motor recovery and independence after sensorimotor-complete cervical spinal cord injury. Neurorehabil Neural Repair 2012;26:1064-1071.

10. Steeves JD, Kramer JK, Fawcett JW, et al. Extent of spontaneous motor recovery after traumatic cervical sensorimotor complete spinal cord injury. Spinal Cord 2011;49:257-265.

11. Organization WH. International Classification of Functioning, Disability and Health (ICF) [online]. Available at: http://www.who.int/.

12. Hudak PL, Amadio PC, Bombardier C. Development of an upper extremity outcome measure: the DASH (disabilities of the arm, shoulder and hand) [corrected]. The Upper Extremity Collaborative Group (UECG). Am J Ind Med 1996;29:602-608. 
13. Kalsi-Ryan S, CurtA, Fehlings MG, Verrier MC. Assessment of the Hand in Tetraplegia Using the Graded Redefined Assessment of Strength, Sensibility and Prehension (GRASSP): Impairment Versus Function. Top Spinal Cord Inj Rehabil 2009;14:34-46.

14. Kalsi-Ryan S, Beaton D, Curt A, et al. The Graded Redefined Assessment of Strength Sensibility and Prehension: reliability and validity. J Neurotrauma 2012;29:905-914.

15. Husted JA, Cook RJ, Farewell VT, Gladman DD. Methods for assessing responsiveness: a critical review and recommendations. J Clin Epidemiol 2000;53:459-468.

16. Guyatt G, Walter S, Norman G. Measuring change over time: assessing the usefulness of evaluative instruments. Journal of chronic diseases 1987;40:171-178.

17. Deyo RA, Diehr P, Patrick DL. Reproducibility and responsiveness of health status measures. Statistics and strategies for evaluation. Controlled clinical trials 1991;12:142S-158S.

18. Beaton DE, Bombardier C, Katz JN, et al. Looking for important change/differences in studies of responsiveness. OMERACT MCID Working Group. Outcome Measures in Rheumatology. Minimal Clinically Important Difference. J Rheumatol 2001;28:400-405.

19. Velstra IM, Curt A, Frotzler A, et al. Changes in Strength, Sensation, and Prehension in Acute Cervical Spinal Cord Injury: European Multicenter Responsiveness Study of the GRASSP. Neurorehabil Neural Repair 2015.

20. Studenski S, Hayes RP, Leibowitz RQ, et al. Clinical Global Impression of Change in Physical Frailty: development of a measure based on clinical judgment. Journal of the American Geriatrics Society 2004;52:1560-1566.

21. Yarkony GM, Roth EJ, Heinemann AW, Lovell L, Wu YC. Functional skills after spinal cord injury rehabilitation: three-year longitudinal follow-up. Arch Phys Med Rehabil 1988;69:111-114.

22. Wirth B, van Hedel HJ, Kometer B, Dietz V, Curt A. Changes in activity after a complete spinal cord injury as measured by the Spinal Cord Independence Measure II (SCIM II). Neurorehabil Neural Repair 2008;22:145-153.

23. Waters RL, Adkins RH, Yakura JS, Sie I. Motor and sensory recovery following incomplete tetraplegia. Arch Phys Med Rehabil 1994;75:306-311.

24. Waters RL, Adkins RH, Yakura JS, Sie I. Motor and sensory recovery following complete tetraplegia. Arch Phys Med Rehabil 1993;74:242-247.

25. Marino RJ, Ditunno JF, Jr., Donovan WH, Maynard F, Jr. Neurologic recovery after traumatic spinal cord injury: data from the Model Spinal Cord Injury Systems. Arch Phys Med Rehabil 1999;80:1391-1396.

26. Ditunno JF, Jr., Cohen ME, Hauck WW, Jackson AB, Sipski ML. Recovery of upper-extremity strength in complete and incomplete tetraplegia: a multicenter study. Arch Phys Med Rehabil 2000;81:389-393.

27. Burns AS, Ditunno JF. Establishing prognosis and maximizing functional outcomes after spinal cord injury: a review of current and future directions in rehabilitation management. Spine (Phila Pa 1976) 2001;26:S137-145.

28. Tanadini LG, Steeves JD, Hothorn T, et al. Identifying Homogeneous Subgroups in Neurological Disorders: Unbiased Recursive Partitioning in Cervical Complete Spinal Cord Injury. Neurorehabil Neural Repair 2014;28:507-515.

29. Marino RJ, Burns S, Graves DE, Leiby BE, Kirshblum S, Lammertse DP. Upper- and lowerextremity motor recovery after traumatic cervical spinal cord injury: an update from the national spinal cord injury database. Arch Phys Med Rehabil 2011;92:369-375. 
30. Catz A, Itzkovich M, Agranov E, Ring H, Tamir A. SCIM--spinal cord independence measure: a new disability scale for patients with spinal cord lesions. Spinal Cord 1997;35:850-856.

31. Land NE, Odding E, Duivenvoorden HJ, Bergen MP, Stam HJ. Tetraplegia Hand Activity Questionnaire (THAQ): the development, assessment of arm-hand function-related activities in tetraplegic patients with a spinal cord injury. Spinal Cord 2004;42:294-301.

32. Post MW, Van Lieshout G, Seelen HA, Snoek GJ, ljzerman MJ, Pons C. Measurement properties of the short version of the Van Lieshout test for arm/hand function of persons with tetraplegia after spinal cord injury. Spinal Cord 2006;44:763-771.

33. Spooren Al, Janssen-Potten YJ, Post MW, Kerckhofs E, Nene A, Seelen HA. Measuring change in arm hand skilled performance in persons with a cervical spinal cord injury: responsiveness of the Van Lieshout Test. Spinal Cord 2006;44:772-779.

34. Fattal C. Motor capacities of upper limbs in tetraplegics: a new scale for the assessment of the results of functional surgery on upper limbs. Spinal Cord 2004;42:80-90.

35. Fattal C, Thery JM, Micallef JP. [Validation of the motor capacities scale: a specific evaluation of manual abilities in tetraplegics who undergo functional surgery of the upper limbs]. Annales de readaptation et de medecine physique : revue scientifique de la Societe francaise de reeducation fonctionnelle de readaptation et de medecine physique 2004;47:537-545.

36. Marino RJ, Patrick M, Albright W, et al. Development of an objective test of upper-limb function in tetraplegia: the capabilities of upper extremity test. Am J Phys Med Rehabil 2012;91:478486.

37. Marino RJ, Shea JA, Stineman MG. The Capabilities of Upper Extremity instrument: reliability and validity of a measure of functional limitation in tetraplegia. Arch Phys Med Rehabil 1998;79:1512-1521.

38. Wuolle KS, Van Doren CL, Thrope GB, Keith MW, Peckham PH. Development of a quantitative hand grasp and release test for patients with tetraplegia using a hand neuroprosthesis. J Hand Surg Am 1994;19:209-218.

39. Kirshblum SC, Waring W, Biering-Sorensen F, et al. Reference for the 2011 revision of the International Standards for Neurological Classification of Spinal Cord Injury. J Spinal Cord Med 2011;34:547-554.

40. Krassioukov A, Wolfe DL, Hsieh JT, Hayes KC, Durham CE. Quantitative sensory testing in patients with incomplete spinal cord injury. Arch Phys Med Rehabil 1999;80:1258-1263.

41. Jonsson M, Tollback A, Gonzales H, Borg J. Inter-rater reliability of the 1992 international standards for neurological and functional classification of incomplete spinal cord injury. Spinal Cord 2000;38:675-679.

42. Hayes KC, Wolfe DL, Hsieh JT, Potter PJ, Krassioukov A, Durham CE. Clinical and electrophysiologic correlates of quantitative sensory testing in patients with incomplete spinal cord injury. Arch Phys Med Rehabil 2002;83:1612-1619.

43. Cohen ME, Sheehan TP, Herbison GJ. Content validity and reliability of the International Standards for Neurological Classification of Spinal Cord Injury. Top Spinal Cord Inj Rehabil 1996:15-31.

44. Furlan JC, Fehlings MG, Tator $\mathrm{CH}$, Davis AM. Motor and sensory assessment of patients in clinical trials for pharmacological therapy of acute spinal cord injury: psychometric properties of the ASIA Standards. J Neurotrauma 2008;25:1273-1301. 
45. Hothorn T, Hornik K, Zeileis A. Unbiased recursive partitioning: a conditional inference framework. J Comput Graph Stat 2006;15:651-674.

46. Singh A, Tetreault L, Kalsi-Ryan S, Nouri A, Fehlings MG. Global prevalence and incidence of traumatic spinal cord injury. Clinical epidemiology 2014;6:309-331.

47. Fawcett JW, Curt A, Steeves JD, et al. Guidelines for the conduct of clinical trials for spinal cord injury as developed by the ICCP panel: spontaneous recovery after spinal cord injury and statistical power needed for therapeutic clinical trials. Spinal Cord 2007;45:190-205.

48. Velstra IM, Bolliger M, Tanadini LG, et al. Prediction and Stratification of Upper Limb Function and Self-Care in Acute Cervical Spinal Cord Injury With the Graded Redefined Assessment of Strength, Sensibility, and Prehension (GRASSP). Neurorehabil Neural Repair 2014;28:632642.

49. van Middendorp JJ, Hosman AJ, Donders AR, et al. A clinical prediction rule for ambulation outcomes after traumatic spinal cord injury: a longitudinal cohort study. Lancet 2011;377:10041010.

50. Zorner B, Blanckenhorn WU, Dietz V, Curt A. Clinical algorithm for improved prediction of ambulation and patient stratification after incomplete spinal cord injury. J Neurotrauma 2010;27:241-252.

51. Wilson JR, Grossman RG, Frankowski RF, et al. A clinical prediction model for long-term functional outcome after traumatic spinal cord injury based on acute clinical and imaging factors. J Neurotrauma 2012;29:2263-2271.

52. Curt A, Dietz V. Traumatic cervical spinal cord injury: relation between somatosensory evoked potentials, neurological deficit, and hand function. Arch Phys Med Rehabil 1996;77:48-53.

53. Kuhn F, Halder P, Spiess MR, Schubert M. One-year evolution of ulnar somatosensory potentials after trauma in 365 tetraplegic patients: early prediction of potential upper limb function. $\mathrm{J}$ Neurotrauma 2012;29:1829-1837.

54. Kalsi-Ryan S, Beaton D, Curt A, Popovic MR, Verrier MC, Fehlings MG. Outcome of the upper limb in cervical spinal cord injury: Profiles of recovery and insights for clinical studies. J Spinal Cord Med 2014;37:503-510.

55. Scott SC, Goldberg MS, Mayo NE. Statistical assessment of ordinal outcomes in comparative studies. J Clin Epidemiol 1997;50:45-55.

56. Velozo CA, Kielhofner G, Lai JS. The use of Rasch analysis to produce scale-free measurement of functional ability. Am J Occup Ther 1999;53:83-90.

57. Catz A, Itzkovich M, Tesio L, et al. A multicenter international study on the Spinal Cord Independence Measure, version III: Rasch psychometric validation. Spinal Cord 2007;45:275291.

58. Doll U, Maurer-Burkhard B, Spahn B, Fromm B. Functional hand development in tetraplegia. Spinal Cord 1998;36:818-821.

59. Harvey L, Baillie R, Bronwyn R, Simpson D, Pironello D, Glinsky J. Does three months of nightly splinting reduce the extensibility of the flexor pollicis longus muscle in people with tetraplegia? Physiother Res Int 2007;12:5-13.

60. Popovic MR, Thrasher TA, Adams ME, Takes V, Zivanovic V, Tonack MI. Functional electrical therapy: retraining grasping in spinal cord injury. Spinal Cord 2006;44:143-151. 
61. Popovic MR, Kapadia N, Zivanovic V, Furlan JC, Craven BC, McGillivray C. Functional electrical stimulation therapy of voluntary grasping versus only conventional rehabilitation for patients with subacute incomplete tetraplegia: a randomized clinical trial. Neurorehabil Neural Repair 2011;25:433-442.

62. Kohlmeyer KM, Hill JP, Yarkony GM, Jaeger RJ. Electrical stimulation and biofeedback effect on recovery of tenodesis grasp: a controlled study. Arch Phys Med Rehabil 1996;77:702-706.

63. Beekhuizen KS, Field-Fote EC. Massed practice versus massed practice with stimulation: effects on upper extremity function and cortical plasticity in individuals with incomplete cervical spinal cord injury. Neurorehabil Neural Repair 2005;19:33-45.

64. Beekhuizen KS, Field-Fote EC. Sensory stimulation augments the effects of massed practice training in persons with tetraplegia. Arch Phys Med Rehabil 2008;89:602-608.

65. Johanson ME, Hentz VR, Smaby N, Murray WM. Activation of brachioradialis muscles transferred to restore lateral pinch in tetraplegia. J Hand Surg Am 2006;31:747-753.

66. Laffont I, Hoffmann G, Dizien O, Revol M, Roby-Brami A. How do C6/C7 tetraplegic patients grasp balls of different sizes and weights? Impact of surgical musculo-tendinous transfers. Spinal Cord 2007;45:502-512.

67. Lo IK, Turner R, Connolly S, Delaney G, Roth JH. The outcome of tendon transfers for C6spared quadriplegics. J Hand Surg [Br] 1998;23:156-161.

68. Meiners T, Abel R, Lindel K, Mesecke U. Improvements in activities of daily living following functional hand surgery for treatment of lesions to the cervical spinal cord: self-assessment by patients. Spinal Cord 2002;40:574-580.

69. Rothwell AG, Sinnott KA, Mohammed KD, Dunn JA, Sinclair SW. Upper limb surgery for tetraplegia: a 10-year re-review of hand function. J Hand Surg Am 2003;28:489-497.

70. Wangdell J, Friden J. Satisfaction and performance in patient selected goals after grip reconstruction in tetraplegia. J Hand Surg Eur Vol 2010;35:563-568.

71. Sinnott KA, Dunn JA, Rothwell AG, Hall AS, Post MW. The development of the NZ-based international upper limb surgery registry. Spinal Cord 2014;52:611-615. 

Summary 
The goal of rehabilitation in general is regaining and/or maintaining functionality by decreasing the consequences of health conditions. For this reason, feasible, reliable, valid and responsive outcome measures are needed to provide insight into the underlying causes. For cervical spinal cord injury $(\mathrm{SCl})$ however, research in upper limb function outcome is limited. Moreover, the course of cervical $\mathrm{SCl}$ shows large variability regarding recovery, lesion level and density, which makes the interpretation of clinical findings and research challenging.

Chapter 1 of this thesis reports that few outcome measures have been developed for cervical $\mathrm{SCl}$ and that they have limited psychometric properties. Thus, there is a clear need for valid, reliable and responsive outcome measures in cervical $\mathrm{SCl}$, in order to assess upper limb function accurately.

The overall aim of this $\mathrm{PhD}$ thesis is therefore to study the assessment, evaluation and prediction of upper limb function up to one year post injury using the Graded and Redefined Assessment of Strength, Sensibility and Prehension (GRASSP) in individuals with cervical SCI.

Chapter 2 presents a systematic review of the literature on the availability of current outcome measures for upper extremity function in the four populations: (1) peripheral upper extremity conditions, (2) rheumatologic diseases, (3) stroke and (4) tetraplegia. Seventeen most frequently used outcome measures were identified and classified according the International Classification of Functioning, Disability, and Health (ICF). For each outcome measure a description of the concept, operationalisation into variables and instruments as well as information on the reliability and responsiveness is given. The outcome measures show large variability with regard to the areas of functioning and disability addressed. Reliability and responsiveness data are missing for some outcome measures or for certain populations for which they have been used. Thus, the overview table (table 2) in this chapter can be used to facilitate the selection process of outcome measures for investigations or clinical practice. Furthermore, the table provides an indication of the areas of upper extremity outcome measures in which future research is needed. In addition, the findings in this chapter show that research with a wider focus is needed to encompass the multifaceted problems of upper extremity function. It is therefore important that outcome measures related to upper extremity function capture the entire spectrum of functioning and disability.

This chapter provides clinicians and researchers with a guide for the selection of the most appropriate outcome measure for their clinical population or research question, 
taking into consideration ICF based content validity ("what do the outcome measures address?"), reliability, and responsiveness.

Due to the limited research on upper limb function outcome measures in cervical $\mathrm{SCI}$ an international research team developed the GRASSP. The GRASSP is an outcome measure that quantifies upper limb impairment changes, and how they contribute to complex upper limb tasks. In chapter 3, a prospective study of individuals with acute cervical SCI up to 1 year post injury investigated (1) the responsiveness of the GRASSP subtests, (2) the responsiveness of the GRASSP subtests compared and related to the upper extremity motor score (UEMS) and light touch (LT) scores according to the International Standards of Neurological Classification of Spinal Cord Injury (ISNCSCI) and the spinal cord independence self-care subscale (SCIM-SS), (3) the clinical appreciation of changes in GRASSP and SCIM-SS by using a clinician-rated outcome measure (CROM), and (4) recovery profiles in GRASSP strength and prehension. The outcome measures were assessed at 1, 3, 6 and 12 months after cervical SCI. Seventy-four individuals with acute cervical SCI from 5 European SCI centers were included for this study. The GRASSP subtests proved responsive (standardized response mean [SRM] ranged from 0.79 to 1.48 for strength, 0.50 to 1.03 for prehension, and 0.14 to 0.64 for sensation) between all examination time points. In comparison, UEMS and LT showed lower responsiveness (SRM UEMS ranged from 0.69 to 1.29 and SRM LT ranged from 0.30 to -0.13). All GRASSP subtests revealed significant, moderate-to-excellent correlations with UEMS, LT and SCIM-SS at each time point, and the changes in GRASSP subtests and SCIM-SS were in accordance with the CROM. The large SRMs and the agreement of the GRASSP with the clinicians' ratings (CROM) reflect great clinical relevance. The GRASSP prehension and motor recovery was greatest between 1 and 3 months.

In conclusion, the GRASSP showed excellent responsiveness, detecting distinct changes in strength and prehension relating to the severity of cervical SCI. It detected clinically significant changes complimentary to the ISNCSCI and SCIM-SS assessments.

The study in chapter 4 compared the epicritic sensation assessed by LT, SemmesWeinstein monofilament (SWM) and electrical perception threshold (EPT) across cervical dermatomes (C3-C8) in individuals with cervical SCI. A total of 300 left- and right-sided dermatomes were tested for each outcome measure in 25 individuals with tetraplegia 6 months after cervical SCI. The percentage agreement between classifications according to LT and SWM/EPT ranged from $95.5 \%$ to $36.2 \%$. The degree of agreement showed considerably variable kappa coefficients $(-0.1 \geq \mathrm{kw} \leq 0.7)$ for each dermatome between C3 and C8. 
In conclusion, the additional measurements of epicritic sensation by SWM or EPT increased sensitivity by detecting and quantifying differences in sensory thresholds above, at and below the LT level of injury.

There is inherent heterogeneity within individuals suffering from cervical $\mathrm{SCl}$, and early prediction of upper limb function and self-care is challenging. As a result, considerable uncertainty exists regarding the prediction of functional outcome following cervical $\mathrm{SCI}$ within 1 year of injury. Therefore, the study in chapter 5 evaluated the value of GRASSP in predicting upper limb function and self-care outcomes in individuals with cervical SCI. A prospective longitudinal multicenter study was performed. Data from the GRASSP, the SCIM III, and the American Spinal Injury Association (ASIA) Impairment Scale (AIS) were recorded at 1, 6, and 12 months after cervical SCI. For prediction of functional outcome at 6 and 12 months, a logistic regression model, receiver operating characteristics (ROC) and unbiased recursive partitioning conditional inference tree (URP-CTREE) were used with 8 different predictor variables. Sixty-one individuals were included for analysis. Logistic regression analysis, ROC analysis, and URP-CTREE all revealed that the motor scoring within GRASSP is the strongest predictor for upper limb function and self-care outcomes. Moreover, the combination of the motor scoring with other predictors, such as qualitative grasping (QIG) and quantitative grasping (QtG), improved outcome prediction. This combination supports the use of GRASSP in the assessment of rehabilitation as well as in interventional clinical trials, which seek to detect both subtle and clinical meaningful changes. In addition, URP-CTREE provided useful information on the distribution of different outcomes even in a heterogeneous group across a wide spectrum of neurological recovery. In conclusion, the GRASSP at 1 month accurately predicted upper limb function and selfcare outcomes at 6 and 12 months after cervical SCI. URP-CTREE revealed the distribution of outcome categories and can be used to predict cohorts with homogenous outcomes.

Evidence was presented that the GRASSP allows the precise identification of recovery profiles and accurately predicts upper limb function and self-care in acute tetraplegia. However, there was still limited evidence regarding the influence of each individual muscle or muscle group on the prediction of upper limb function and activities of daily living (ADLs). Furthermore, the effect of specific grasp patterns, as described in the GRASSP, on the prediction of upper limb function and functional outcome had not yet been investigated. In chapter 6 therefore, it was determined which single or combined upper limb muscles as defined by the ISNCSCI-UEMS and the GRASSP, best predict upper limb function and independence in ADLs. Furthermore, the predictive value of qualitative grasp movements 
(QIG) on upper limb function in individuals with acute tetraplegia was assessed. In this study ISNCSCI, GRASSP and SCIM III scores were recorded at 1 and 6 months after SCI. For the prediction of upper limb function and ADLs, a logistic regression model and URP-CTREE were used with 10 different muscle predictor variables and 2 ISNCSCI predictor variables, motor level of injury (MLI) and AIS. Logistic regression and URP-CTREE revealed that a combination of ISNCSCI and GRASSP muscles (to a maximum of four) demonstrated the best prediction (specificity and sensitivity ranged from $81.8 \%$ to $96.0 \%$ ) of upper limb function and identified homogenous outcome cohorts at 6 months. The URP-CTREE model with the QIG predictors for upper limb function showed similar results. The AIS and MLI predictor variables were not chosen in the URP-CTREE model. Therefore, AIS grades and MLI might not be appropriate for stratification in clinical studies or trials. Prediction of upper limb function can be accurately achieved through a combination of defined, specific upper limb muscles assessed in the ISNCSCI and GRASSP. These limited muscle predictors are ideal indices for stratifying patient groups and a good proxy for favourable and unfavourable outcomes irrespective of AIS and MLI. In addition, the combination of a limited number of proximal and distal muscles along with QIG represents a quick and easy assessment tool for clinical decision making during rehabilitation interventions and clinical trials. In conclusion, the predictive value of standard ISNCSCI-UEMS for upper limb function can be improved significantly with the addition of single GRASSP predictors.

Finally, in chapter 7 , the main findings of this thesis are presented and discussed, and recommendations are made for further research.

\section{Overall conclusion}

The work contained in this $\mathrm{PhD}$ thesis provides advanced insights and useful data regarding upper limb function outcome in cervical SCI. Evidence was presented that the GRASSP provides information on multifaceted domains (e.g. strength, sensation and prehension) and allows to detect both subtle and clinical meaningful changes in upper limb function. Furthermore, the GRASSP can accurately predict upper limb function and ADLs, even in a heterogeneous group of individuals across a wide spectrum of neurological recovery. This supports the use of the GRASSP in the assessment of rehabilitation outcome as well as in clinical studies and trials. 

Samenvatting 
Revalidatie heeft als doel om bij mensen met een gezondheidsprobleem het dagelijks functioneren te herstellen of te behouden. Bruikbare, betrouwbare en valide meetinstrumenten, die ook veranderingen in de gezondheidstoestand kunnen vastleggen, zijn nodig om binnen het revalidatieproces inzicht te krijgen in de onderliggende oorzaken van het verminderd functioneren.

Bij mensen met een cervicale dwarslaesie is de arm- en handfunctie aangedaan en daarom is de behandeling van de bovenste extremiteiten van groot belang. Er is echter beperkt onderzoek gedaan naar uitkomstmaten van arm- en handfunctie. Verder kan de uitingsvorm en het verloop van een cervicale dwarslaesie heel verschillend zijn, wat voornamelijk wordt bepaald door variatie in de hoogte, de ernst en het herstel van de laesie. Deze verschillen maken het lastig om de verschijnselen en testresultaten bij individuele patiënten te interpreteren en het bemoeilijkt ook de interpretatie van onderzoeksgegevens.

Hoofdstuk 1 van dit proefschrift laat zien dat er maar weinig meetinstrumenten voor mensen met een cervicale dwarslaesie zijn ontwikkeld en dat de meetinstrumenten die er wel zijn, geen, beperkte of matige klinimetrische meeteigenschappen bezitten. Om arm- en handfunctie nauwkeurig te kunnen weergeven is het daarom nodig om voor deze patiënten betrouwbare, valide en responsieve meetinstrumenten te ontwikkelen of om bij bestaande instrumenten de meeteigenschappen verder te onderzoeken. Het hoofddoel van dit proefschrift is om bij mensen met een cervicale dwarslaesie de arm- en handfunctie te meten, te evalueren en te voorspellen tot een jaar na het ontstaan van de laesie, middels de 'Graded and Redefined Assessment of Strength, Sensibility and Prehension' (GRASSP).

Hoofdstuk 2 beschrijft een systematische literatuurstudie naar de beschikbaarheid van meetinstrumenten voor arm- en handfunctie in vier verschillende patiëntengroepen: 1) aandoeningen van schouder-arm-hand; 2) reumatische ziekten; 3) herseninfarct; 4) tetraplegie. Met tetraplegie wordt een verlamming van zowel beide armen als beide benen bedoeld, zoals die kan ontstaan na een cervicale dwarslaesie. Er bleken 17 veelgebruikte meetinstrumenten te zijn, die volgens de 'International Classification of Functioning, Disability, and Health' (ICF) ingedeeld kunnen worden. Voor ieder meetinstrument is het onderliggend concept, de uit te voeren metingen en informatie over betrouwbaarheid en responsiviteit voor veranderingen gegeven. Samenvattend kan gezegd worden dat deze veelgebruikte meetinstrumenten onderling behoorlijke verschillen vertonen in concept en de metingen. Tevens mist er vaak informatie over de klinimetrische eigenschappen. Het overzicht (tabellen 2.1 en 2.2 in hoofdstuk 2) kan gebruikt worden om de keuze voor een meetinstrument in de klinische praktijk of voor een studie te vergemakkelijken. 
Vanwege het gebrek aan goede meetinstrumenten voor de arm- en handfunctie voor mensen met een cervicale dwarslaesie, heeft een internationaal onderzoeksteam de 'Graded and Redefined Assessment of Strength (spierkracht), Sensibility (tastzin) and Prehension (reiken, grijpen, loslaten en manipuleren)' (GRASSP) ontwikkeld. De GRASSP is een meetinstrument dat veranderingen in functie van de arm en hand kan vastleggen, in relatie tot complexe vaardigheden van de arm en hand. In hoofdstuk 3 wordt een studie beschreven bij 74 patiënten met een acute cervicale dwarslaesie die op 1, 3, 6 en 12 maanden na de dwarslaesie zijn onderzocht. Onderzocht is of de GRASSP responsief is voor veranderingen over die tijd, in vergelijking met andere gestandaardiseerde tests (zoals de 'upper extremity motor score' (UEMS), de gevoeligheid voor lichte aanraking ('light touch', LT) die volgens internationale richtlijnen (ISNCSCI) getest worden en een schaal voor het niveau van zelfstandigheid bij activiteiten van de zelfverzorging (SCIMSS)). Verder is er gekeken naar het herstelpatroon van de onderdelen 'spierkracht' en 'prehension' in de GRASSP en is er gekeken in hoeverre veranderingen in de GRASSP en SCIM-SS overeenkwamen met veranderingen volgens het oordeel van behandelaars.

De GRASSP is uitstekend in staat is om tot een jaar na de dwarslaesie klinisch relevante veranderingen in arm- en handfunctie te meten. GRASSP spierkracht en prehension herstel waren het grootst tussen 1-3 maanden na het ontstaan van de dwarslaesie en zijn gerelateerd aan de ernst van de dwarslaesie. De metingen van de GRASSP bleken van toevoegende waarde te zijn bij de bestaande standaardmetingen (INSCSCI en SCIM-SS).

Bij mensen met een cervicale dwarslaesie treden niet alleen spierparesen op, maar bestaat er ook uitval van gevoel en tastzin. In hoofdstuk 4 worden drie verschillende meetmethoden getest, die gebruikt worden om te onderzoeken wat mensen met een tetraplegie nog kunnen waarnemen met de huid van schouder arm en hand (op de dermatomen tussen C3-C8): de gevoeligheid voor lichte aanraking (LT), de Semmes-Weinstein monofilament (SWM) test en de grenswaarde om een elektrische prikkeling nog net waar te nemen (EPT). Bij 25 patiënten met tetraplegie zijn, 6 maanden na de laesie, in totaal 300 huidgebieden getest, zowel links als rechts.

Het percentage van overeenstemming tussen de LT en SWM/EPT testuitslagen varieerde van $95.5 \%$ tot $36.2 \%$. De meetinstrumenten SWM en EPT zijn gevoeliger om tastzin te meten dan de LT, doordat er onder, boven en op het LT-niveau van de laesie nog tastzin met EPT en SWM gedetecteerd werd. Dat betekent dat SWM of EPT een goede aanvulling is op LT. 
Er bestaat een grote variabiliteit in het verloop na het doormaken van een cervicale dwarslaesie. Voor de patiënt en voor de revalidatie zou het goed zijn als het verloop na de dwarslaesie zou kunnen worden voorspeld om daarmee de patiënten goed te informeren over hun vooruitgang. In hoofdstuk 5 staat een studie beschreven, waarin onderzocht is of je met de GRASSP-test de arm- en handfunctie en de mate van zelfstandigheid bij zelfverzorging een jaar na de dwarslaesie kan voorspellen. In de studie is bij 61 patiënten 1 maand, 6 en 12 maanden na de dwarslaesie, de GRASSP, SCIM-SS en de American Spinal Injury Association (ASIA) Impairment Scale (AIS) (Gemodificeerde Frankel Classificatie om de mate van stoornis aan te geven) afgenomen. De spierkracht in de GRASSP bleek de beste voorspeller voor het verloop van arm- en handfunctie en zelfverzorging. De voorspelling werd nog beter als de spierkracht met het kwalitatieve grijpen en/of kwantitatieve grijpen werd gecombineerd. Kwalitatief grijpen werd getest met de 'qualitative grasping subtest" (QIG) waarin verschillende grijppatronen zoals de cilindergreep, pincetgreep en lateraalgreep uitgevoerd worden zonder dat een voorwerp gepakt wordt. Kwantitatief grijpen werd getest met de 'quantitative grasping subtest' (QtG), waarin zes verschillende taken uitgevoerd worden, bijvoorbeeld water uit een flesje in een glas schenken en moeren op een boutje draaien, waarbij gekeken en beoordeeld wordt met welke greep de patiënt het voorwerp pakt. Bovendien leverde de beslisboomanalyse (decision tree analysis: URP-CTREE) belangrijke informatie op over de verdeling van verschillende homogene uitkomsten, zelfs uit een heterogene groep van patiënten waarvan het neurologische herstel zo verschillend is. In conclusie kan worden gesteld dat de GRASSP afgenomen op 1 maand na de dwarslaesie nauwkeurig het verloop van de armen handfunctie en zelfverzorging tot 1 jaar na de dwarslaesie kan voorspellen. Daarnaast laat de URP-CTREE-analyse zien dat de verdeling van uitkomsten uitstekend gebruikt kan worden om cohorten met homogene uitkomsten te voorspellen. Dit was tot nu toe erg moeilijk bij cervicale dwarslaesie en was nooit nader onderzocht. De bovengenoemde factoren ondersteunen het gebruik van de GRASSP in revalidatie en interventiestudies.

Uit de hoofdstukken 3 en 5 is gebleken dat de GRASSP geschikt is om bij mensen met een acute cervicale dwarslaesie nauwkeurig herstelpatronen te beschrijven en om de arm- en handfunctie en zelfverzorging precies te voorspellen. De daaropvolgende vraag is in hoeverre de functie van individuele spieren of spiergroepen, zoals beschreven in de GRASSP en ISNCSCI-UEMS, samenhangt met latere arm- en handfunctie en zelfstandigheid in de zelfverzorging en mobiliteit. Ook was het nog de vraag in hoeverre QIG, zoals beschreven in de GRASSP en het motorische niveau van de laesie (MLI) en de ernst van 
de laesie (AIS), samenhangt met latere functionaliteit. In het onderzoek in hoofdstuk 6 is beschreven dat een combinatie van maximaal vier verschillende spieren of spiergroepen predictoren uit ISNCSCI-UEMS en GRASSP de arm- en handfunctie 6 maanden later nauwkeurig kan voorspellen. Bovendien kan het testen van deze spieren heel bruikbaar zijn om te informeren over de juiste behandeling in de revalidatie. De QIG-predictoren leverden dezelfde resultaten op. Bij alle analyses werden de MLI- en AIS-predictoren niet in het model opgenomen. Dus een beperkt aantal spieren of spiergroepen alsook een eenvoudige en snelle test zoals QIG kunnen uitstekend de arm- en handfunctie voorspellen en patiënten in goede en slechte uitkomsten stratificeren onafhankelijk van MLI en AIS. Er kan geconcludeerd worden dat de voorspellende waarde van ISNCSCI-UEMS voor arm- en handfunctie significant verbeterd kan worden door GRASSP-predictoren toe te voegen.

In hoofdstuk 7 worden de belangrijkste bevindingen van dit proefschrift gepresenteerd en besproken, en worden aanbevelingen gedaan voor verder onderzoek. Het werk in dit proefschrift geeft nieuw inzicht en waardevolle data over de arm- en handfunctie bij mensen met een cervicale dwarslaesie. De GRASSP is een relatief nieuwe test die informatie geeft over verschillende domeinen van arm- en handfunctie, zoals spierkracht, tastzin en de vaardigheid om naar voorwerpen te reiken, te grijpen, los te laten en te manipuleren. De GRASSP is in staat om de soms kleine maar klinisch belangrijke veranderingen in arm- en handfunctie te meten, die belangrijk zijn voor functieherstel. Met de uitslag van de GRASSP kan ook het verloop van arm- en handfunctie en zelfverzorging nauwkeurig voorspeld worden, zelfs als bij cervicale dwarslaesie de gevolgen voor patiënten zo verschillend kunnen zijn. Dit ondersteunt het gebruik van de GRASSP voor het meten van de uitkomsten in de revalidatie van mensen met een cervicale dwarslaesie en ondersteunt tevens het gebruik van de GRASSP in klinische studies. 



\section{Danksagungen Acknowledgements \\ Dankwoord}


Voller Freude darf ich die nun abgeschlossene PhD-Arbeit in einem Buch der Öffentlichkeit vorlegen. In den letzten Jahren habe ich mein Wissen im Bereich des wissenschaftlichen Arbeitens erweitert. Die PhD-Arbeit war für mich ein Zusammenspiel von Organisieren, Koordinieren, Überprüfen, Führen, Schreiben, Analysieren, Durchhalten, Zuhören, exaktem und effizientem Arbeiten, Relativieren, Präsentieren und vielem mehr. Symbolisch habe ich in dieser Zeit immer den Hausberg von Luzern, den Pilatus, mit all seinen Kontrasten vor Augen gehabt. Im dicksten Nebel oder sehr klar mit grosszügiger Aussicht; Grautöne oder eine hervorragende Farbenpracht; anspruchsvollen Passagen oder langsam erholend auf vertrauten Wegen an Höhe gewinnen; absteigen und heimkehren oder den Gipfel anstreben. Ich hatte ein PhD-Ziel vor Augen und musste dieses je nach Gegebenheiten immer wieder ein wenig anpassen oder einen ganz neuen Weg einschlagen. Jetzt sind die vielen spannenden Jahre voll intensiver Arbeit vorbei, und ich bin oben auf dem Gipfel angekommen. Ebenso freut es mich, all jenen zu danken, die einen wertvollen Beitrag geliefert haben, um mir die Erstellung meiner PhD-Arbeit zu ermöglichen.

Allen voran den Probanden, die an den Studien teilgenommen haben. Ich bin euch sehr dankbar, dass jetzt die Resultate vorliegen, die die klinische Rehabilitation und Forschung im Bereich Tetraplegie vorangetrieben haben. Ohne euch hätte ich keine Studien und keine Ergebnisse. Vielen Dank für die schönen und wertvollen Begegnungen.

Grote dank ben ik verschuldigd aan mijn promotor Prof.dr. Hans Rietman, die mij in 2011 de kans bood om een PhD-traject aan de Universiteit van Twente te doorlopen. Hoewel ik niet veel in Enschede ben geweest, stond je altijd ongedwongen voor me klaar. Ik wil je heel graag bedanken voor de vrijheid die je me hebt gegeven tijdens het onderzoek, voor je prettige samenwerking en ondersteuning door de jaren heen.

Mein grosser und ganz besonderer Dank geht an meinen Promotor Prof. Dr. Armin Curt, welcher die Grundlage für meine PhD-Arbeit gelegt hat, mir das Vertrauen gegeben hat diese GRASSP Studie in Europa durchzuführen und mir mit seinem Fachwissen zur Seite stand. Ohne deine wertvollen klinischen und wissenschaftlichen Hinweise, konstruktiven Gespräche und kritischem Durchlesen all meiner Manuskripte wäre diese Arbeit nicht entstanden. Bedanken möchte ich mich auch für die Zeit, die du dir genommen hast, wenn ich es gebraucht habe. Danke auch für den Raum den du mir gegeben hast, eine $\mathrm{PhD}$-Arbeit in Kombination mit Arbeit und Mutterschaft zu gestalten. 
Ein sehr grosser Dank geht an meinen Supervisor Dr. Marc Bolliger, der für mich immer Ansprechpartner war und mich in den vergangenen Jahren bei den unterschiedlichsten Aufgaben sehr kompetent beraten und mir bei inhaltlichen sowie methodischen Fragen immer zur Seite stand. Du hast mir die Freiheit gelassen, diese Arbeit nach eigenen Vorstellungen zu entwickeln. Deine offene, lockere, positive und motivierende Art sowie die privaten Gespräche habe ich sehr geschätzt. Sie machten die teilweise sehr langen Tage ein bisschen leichter und angenehmer. Vielen Dank für die lehrreiche Zeit und dein Engagement.

Bedanken möchte ich mich ebenfalls beim ganzen Forschungsteam des Zentrums für Paraplegie der Universitätsklinik Balgrist, vor allem bei Urs Albisser und Claudia Rudhe, für das Durchführen der Messungen in der Universitätsklinik Balgrist und die bereichernden Diskussionen hinsichtlich „GRASSP”, bei René Koller, der am Anfang meiner PhD-Zeit all meine Fragen zu AIS-Klassifikationen sehr kompetent und innerhalb kürzester Zeit beantwortet hat und bei Lorenzo Tanadini für die lehrreichen Statistik-Stunden und Unterstützung beim Recursive Partitioning. A great thank to Tim Killeen who critical read and definitely optimised my last papers.

Ebenfalls möchte ich Diana Sigrist-Nix und Aurelio Tobón für die Durchführung dieser Studie in der Abteilung Ergotherapie und Neurologie im Schweizer Paraplegiker-Zentrum Nottwil (SPZ) danken. Ein sehr grosser Dank geht an Diana Sigrist-Nix, Bart de Kimpe und Friederike Ebert, die mir manchmal GRASSP Messungen abgenommen haben, als auch an Aurelio Tobón und Trudy Röösli für die Durchführung von neurologischen Befunden. Ihr habt mich entlastet und dazu beigetragen, dass nach 14 Monaten Rekrutieren eine hohe Fallzahl mit vielen kompletten Datensets vorhanden war. Herzlichen Dank.

Bedanken möchte ich mich bei Doris Meier und Orpheus Mach, Unfallklinik Murnau, Deutschland, Rudiger Rupp, Orthopädische Universitätsklinik Heidelberg, Deutschland, und Rainer Abel, Zentrum Hohe Warte Bayreuth, Deutschland, für die freundliche Bereitschaft zur Teilnahme und Zusammenarbeit an dieser Studie. Insbesondere danke ich Kristin Hautsch, Petra Schatz und Elke Engelbrecht (Ergotherapie, Bayreuth), Marlis Euler und Ruth Krahl (Ergotherapie, Heidelberg) und Denise Dengg mit den zuständigen GRASSP Therapeutinnen und Therapeuten (Ergotherapie Murnau) für die ausdauernde Durchführung der Datenerhebung. Ihr habt viel Arbeit geleistet und das Projekt wesentlich mitgeprägt. Vielen Dank. 
Ein ganz spezieller Dank geht an meine Kolleginnen und Kollegen Angela Frotzler und das Clinical Trial Unit Team: Gabi Müller-Verbiest, Jörg Krebs, Anja Raab, Stefanie Tesini, Anne Brust und Mathias Guler, die mir zwischendurch Aufgaben abnahmen und meine unterschiedliche Emotionen, vor allem am Ende meiner PhD-Zeit, ertrugen. Die fröhliche Stimmung, die bereichernden Tipps, wissenschaftliche Inputs, konstruktive Gespräche und eure Unterstützung habe ich sehr geschätzt. Herzlichen Dank für eure Bereitschaft, Teile von meiner PhD-Arbeit zu lesen und zu kontrollieren. Die privaten Gespräche und die unterschiedlichsten Anlässe (;) waren wertvolle und sehr willkommene Wegbegleiter. Ich freue mich auf weitere, spannende Zeiten mit euch.

Natürlich möchte ich mich bei ganz vielen (ehemaligen) Mitarbeiterinnen und Mitarbeitern im SPZ Nottwil bedanken, die auf unterschiedlichen Ebenen an meiner PhD-Arbeit mitgeholfen haben: Medizin, Therapie, Pflege, Sekretariat, Planung, Informatik, Bibliothek und Management. Ich könnte alle nennen, aber ich habe Angst jemanden zu vergessen. Hervorheben möchte ich hier Beat Villiger, Michael Baumberger und Hans Peter Gmünder, die dazu beigetragen haben, diese Studie im SPZ Nottwil zu starten und zu implementieren.

Weiterhin möchte ich mich auch bei allen ehemaligen Kolleginnen und Kollegen der Schweizer Paraplegiker Forschung (SPF) und einer ehemaligen Mitarbeiterin der Clinical Trial Unit bedanken. Eure Hilfsbereitschaft auf den unterschiedlichsten Ebenen und eure offene Art, wissenschaftliche Themen zu diskutieren, schätze ich sehr. Insbesondere Alarcos Cieza, Alexandra Rauch, Carolina Ballert, Kathrin Hartmann, Reuben Escorpizo, Teresa Brinkel, Tom Brinkel, Ursina Arnet, Veronika Lay und Wolfgang Segerer. Ebenfalls möchte ich Gerold Stucki und Mirjam Brach für die wissenschaftlichen Erfahrungen, angeregten Diskussionen und Unterstützung während meiner SPF Zeit bedanken.

An dieser Stelle möchte ich mich bei Joëlle, Teresa und Tom für die vielen schönen, ablenkenden und energietankenden Stunden beim Sport, die wir gemeinsam in Nottwil und Umgebung zusammen verbrachten, ganz herzlich bedanken. Mit euch habe ich oft rennend viele gute Gespräche geführt und immer den Kopf auslüften können.

I would like to thank John Steeves for his enriched inputs at the time he was in Switzerland at the Uniklinik Balgrist in Zurich.

I am grateful to the International GRASSP research and design team: Armin Curt, Susan Duff, Michael Fehlings, Sukhvinder Kalsi-Ryan, Claudia Rudhe and Mary C. Verrier, who granted me to use the GRASSP and especially Sukhvinder Kalsi-Ryan for her collaboration on this study in Europe. 
I would like to thank the members of the reading committee: Prof.dr. V. Dietz, Prof.dr. M.W.M. Post, Prof.dr.ir. H.F.J.M. Koopman, Prof.dr.ir. H.J. Hermens and the chairman Prof.dr. G.P.M.R. Dewulf, for their time and evaluation of my PhD thesis.

Nicht minder aufreibend waren die vergangenen Jahren für alle, die indirekt oder direkt an diesem Werk in allen Phasen mit jeder möglichen Unterstützung geholfen haben. Eine PhD-Arbeit schreiben ist nicht wirklich eine soziale Beschäftigung. Ich bedanke mich an dieser Stelle ganz herzlich für eure liebevolle Fürsorge, Unterstützung, Aufmunterungen und euer Verständnis. Ohne eure grossartigen Betreuungseinsätze, hätte mein PhDZeit sicher länger gedauert. Nebenbei habt ihr dafür gesorgt, dass das Leben nicht nur aus Forschung und PhD bestand, sondern dass ich mich jedes Mal freuen konnte auf Freizeitbeschäftigungen wie ein Kino- oder Theaterbesuch, Kaffeetrinken, Essen, Kochen, Backen, Grillieren, Zelten, Biwakieren, kreatives Gestalten, Spielabenden, Plaudern, Ski(hoch)touren, Klettern, Wandern, Biken, Joggen, Skifahren und abkühlende Momente in und auf dem Wasser. Ohne jemanden hervorheben zu wollen, möchte ich folgende Personen namentlich nennen: Sibylle, Thomas, Daniela, Christian, Brigitte, Eveline, Joachim, Sabine, Chrigi, Ursula, Ulrich, Susanne, Silvia, Fladi, Erika, Urs, Markus, sowie die Skitourengruppe „Wicky-Frey”, Regula, Michael, Cornelia, Felix, Jacqueline, Peter, Agnes, Andreas, Anne-Marie und Ernst in der Schweiz; Evelyn, Aad, Mascha, Jaap, Yvonne, Wout, Annelies und David aus der Niederlande.

Auch möchte ich mich bei Antoinette, praktizierende Physiotherapeutin, Mutter von vier Kindern und „best in class Tagesmutter” ganz herzlich bedanken für deine Flexibilität und spontanen Betreuungseinsätze.

Ebenfalls den Grosseltern Käthi und Willy möchte ich herzlich danken, nehmt ihr Quirine hin und wieder zu euch in die Ferien.

Natuurlijk wil ik mijn paranimfen Evelyn en Jaap heel graag bedanken. Hoewel mijn promotieplechtigheid op het moment van dit schrijven nog moet plaats vinden, hebben jullie me nu al geholpen met de laatste loodjes.

Lieve Evelyn, jij bent sinds onze studententijd in Amsterdam een goede vriendin van mij en ik hoop dat ik nog lang deelgenoot van je leven en gezin mag zijn, ondanks dat bij jou de kinderen nu bijna de deur uit zijn. De humor die we samen delen, onze waardevolle vrijetijdsbestedingen, je nuchterheid, gezelligheid en ondersteuning waren zeer welkome en nuttige afleidingen tijdens het schrijven van mijn proefschrift. Daarom sta je vandaag ook naast me als paranimf. 
Lieve Jaap (of beter gezegd, Japie), nu sta ik er dus echt, jij geloofde er altijd in dat het ooit zou gebeuren. Ikzelf heb zo mijn twijfels gehad of het wel wat zou worden. Jij bent door de jaren in Zwitserland als collega verworden tot goede vriend. Het is geruststellend zo iemand als jou als paranimf naast me te hebben. Je bent betrokken geweest bij mijn promotieonderzoek en je vroeg altijd hoe het ging met de voortgang. Daarnaast heb je mij op allerlei manieren ondersteund en geholpen. Zonder je vriendschap, advies en gezamelijke ondernemingen met en zonder familie weet ik niet of ik dit proefschrift had volgehouden.

Lieve pa en ma, jullie hebben mij de bouwstenen gegeven om me te ontplooien en boden me de kansen om te studeren. Jullie hebben me geleerd om door te zetten als je iets graag wilt bereiken en om het positieve uit een situatie te halen. Helaas kan mijn vader dit niet meer meemaken, maar wat zou hij er trots op geweest zijn.

Een dikke merci aan mijn zussen en aanhang alsook de rest van mijn familie voor de interesse, aanmoedigingen en afleiding.

Lieve Quirine, iedere dag geniet ik van je lachen, je stralende grote ogen en onuitputtelijke energie. Heerlijk dat je er bent. Hartelijk dank voor het ontwerpen van de voorkant van dit proefschrift. Veel liefs en een dikke knuffel van mij. xxx 

Curriculum vitae 
Inge-Marie Velstra was born in 1967 on the 1st of May in Apeldoorn, The Netherlands (NL). She completed her secondary education in 1986 at the Myrtus College in Apeldoorn (NL). In 1990, she obtained her Bachelor's degree as an occupational therapist at the College of Amsterdam, Department of Occupational Therapy, Amsterdam (NL).

In 1992, she moved to Switzerland $(\mathrm{CH})$ and was working as a clinician mainly in the field of spinal cord injury (SCl) care in the Swiss Paraplegic Centre Nottwil $(\mathrm{CH})$ and the Swiss Paraplegic Centre, University Hospital Balgrist, Zurich (CH) until 1997. In 1994, she became the group leader of the occupational therapy department, responsible for the $\mathrm{SCl}$ ward at the Uniklinik Balgrist.

In 1997, she started working as a clinician in the sector of peripheral upper limb conditions at the Department of Rheumatology and the Institute of Physical Medicine of the University Hospital Zurich $(\mathrm{CH})$ as the vice head of the occupational therapy department. Additionally, starting in 1998, she was responsible for building up the new hand therapy department and was the head of this department. During those years she has gained experience in management, organisation and teaching and was involved operationally in projects that aimed at improving clinical quality. Due to her professional experience in $\mathrm{SCl}$ care, hand therapy and rehabilitation, and the treatment and assessment of patients, she got interested in developing / improving her scientific skills. Inge-Marie received her Master's degree in Health Service Research at the Erasmus University Rotterdam (NL) in 2005. She conducted her master thesis "Applicability of the willingness-to-pay method to evaluate health effects in hand injury rehabilitation" at the Department of Rheumatology and the Institute of Physical Medicine, University Hospital Zurich $(\mathrm{CH})$ in collaboration with the Department of Physical Medicine and Rehabilitation, University Hospital Munich, Ludwig-Maximillian-University, Germany.

At the end of 2006, Inge-Marie began to work as a research assistant at the Swiss Paraplegic Research Institute in Nottwil (CH).

In 2010, she started her PhD studies in the Clinical Trial Unit of the Swiss Paraplegic Centre in Nottwil $(\mathrm{CH})$. Her promotors were Prof.dr. Hans Rietman, Enschede (NL) and Prof.dr. Armin Curt, Zurich ( $\mathrm{CH})$, and she was supervised by Dr. Marc Bolliger, Zurich $(\mathrm{CH})$.

Inge-Marie continues her research with the focus on upper limb function and outcome research in SCl. Furthermore, she is looking for opportunities and new challenges to contribute to the improvement of quality, efficiency and effectiveness in $\mathrm{SCl}$ research and care. She is still working at the Clinical Trial Unit in Nottwil (CH) and since spring 2015, an important part of her work is monitoring clinical studies at the Swiss Paraplegic Centre, Nottwil (CH). 

RRD Progress-range 
$\mathrm{PhD}$ Theses en andere boeken zijn gepubliceerd in de RRD Progress-range. De volgende boeken zijn gepubliceerd:

Progress 1: J.W.G.A. Pot, H. Boer, W.H. van Harten, H.J. Hermens, E.R. Seydel. Comprehensive Need-Assessment. Ontwikkeling van een meetinstrument voor zorgbehoeften en kwaliteitsbeoordeling door patiënten, Roessingh Research \& Development, The Netherlands, September 1994, ISBN 90-25452-01-2.

Progress 2: N.G.A. van Leerdam, H.J. Hermens. Revalidatietechnologie in Euregio, Roessingh Research \& Development, The Netherlands, July 1995, ISBN 90-75452-02-0.

Progress 3: L. Duda. L.O. van Noort, S. Röseler, B.O.L. Greitemann, W.H. van Harten, N.S. Klazinga. Rehabilitation in Germany and the Netherlands, A comparison of two rehabilitationsystems, Roessingh Research \& Development, The Netherlands, August 1995, ISBN 90-7545203-9.

Progress 4: Hermie J. Hermens, Anand V. Nenen, Gerald Zilvold. Electrophysiological Kinesiology, Proceedings of the 11th congress of the International Society of Electrophysiologie and Kinesiology in Enschede, The Netherlands 1996, Roessingh Research \& Development, The Netherlands, October 1996, ISBN 90-75452-04-7.

Progress 5: Wim H. van Harten. Bouwen aan een kwaliteitssysteem in de revalidatiezorg. Een poging tot constructieve technology assessment van een kwaliteitssysteem in een gezondheidszorginstelling, Roessingh Research \& Development, The Netherlands, December 1997, ISBN 90-75452-07-1.

Progress 6: Gert Baardman, Maarten J. IJzerman. Design and evaluation of a hybrid orthosis for people with paraplegia, Roessingh Research \& Development, The Netherlands, November 1997, ISBN 90-75452-08-X.

Progress 7: Miriam M.R. Hutten. Lumbar Dynamometry: A useful method for assessment of patients with chronic low back pain? Roessingh Research \& Development, The Netherlands, November 1999, ISBN 90-75452-13-6.

Progress 8: A. van der Salm, W.H. van Harten, C.G. B. Maathuis. Ketenkwaliteit Cerebrale Parese Zorg. Een beschrijving van de cerebrale parese zorg en mogelijke verbeteringen hierin, Roessingh Research \& Development, The Netherlands, April 2001, ISBN 90-75452-19-5.

Progress 9: Marc J. Nederhand. Muscle activation patterns in post traumatic neck pain, Roessingh Research \& Development, The Netherlands, March 2003, ISBN 90-75452-27-6.

Progress 10: Michiel J.A. Jannink. Usabillity of custom-made orthopaedic shoes in patients with degenerative disorders of the foot, Roessingh Research \& Development, The Netherlands, September 2004, ISBN 90-75452-28-4. 
Progress 11: Mariëtte Blokhorst. State-dependent factors and attention in whiplash associated disorder, Roessingh Research \& Development, The Netherlands, January 2005, ISBN 90-3652111-4.

Progress 12: Jaap H. Buurke. Walking after stroke co-ordination patterns \& functional recovery, Roessingh Research \& Development, The Netherlands, February 2005, ISBN 90-365-2140-8.

Progress 13: Arjan van der Salm. Spasticity reduction using electrical stimulation in the lower limb of spinal cord injury patients, Roessingh Research \& Development, The Netherlands, October 2005, ISBN 90-365-2253-6.

Progress 14: Govert J. Snoek. Patient preferences for reconstructive interventions of the upper limb in tetraplegia, Roessingh Research \& Development, The Netherlands, December 2005, ISBN 90-365-2255-2.

Progress 15: Joke de Kroon. Therapeutic electrical stimulation of the upper extremity in stroke, Roessingh Research \& Development, The Netherlands, December 2005, ISBN 90-365-2269-2.

Progress 16: $\mathrm{H}$. van Dijk. Motor skill learning, age and augmented feedback. Roessingh Research and Development, The Netherlands, March 2006, ISBN 90-365-2302-9.

Progress 17: C.A.J. Mes. Improving non-optimal results in chronic pain treatment. Roessingh Research and Development, The Netherlands, January 2007, ISBN 90-365-2435-0.

Progress 18: G.E. Voerman. Musculoskeletal neck-shoulder pain: a new ambulant myofeedback intervention approach. Roessingh Research and Development, The Netherlands, March 2007, ISBN 90-365-2460-1.

Progress 19: L.A.C. Kallenberg. Multi-channel array EMG in chronic neck-shoulder pain. Roessingh Research and Development, The Netherlands, March 2007, ISBN 90-365-2459-8.

Progress 20: M.H.A. Huis in 't Veld. Work-related neck-shoulder pain: The role of cognitivebehavioural factors and remotely supervised treatment. Roessingh Research and Development, The Netherlands, December 2007, ISBN 978-90-365-2584-8.

Progress 21: Judith F.M. Fleuren. Assessment of Spasticity: From EMG to patients' perception. Roessingh Research and Development, The Netherlands, October 2009, ISBN 978-90-3652869-6.

Progress 22: Jasper Reenalda. Dynamic sitting to prevent pressure ulcers in spinal cord injured. Roessingh Research and Development, The Netherlands, October 2009, ISBN 978-90-3652884-9. 
Progress 23: G.B. (Gerdienke) Prange. Rehabilitation Robotics: Stimulating restoration of arm function after stroke. Roessingh Research and Development, The Netherlands, October 2009, ISBN 978-90-365-2901-3.

Progress 24: M. Vos-van der Hulst. Prognostic factors and underlying mechanisms in chronic low back pain. Roessingh Research and Development, The Netherlands, November 2009, ISBN 978-90-365-2881-8.

Progress 25: Anke I.R. Kottink-Hutten. Assessment of a two-channel implantable peroneal nerve stimulator post-stroke. Roessingh Research and Development, The Netherlands, February 2010, ISBN: 978-90-365-2959-4.

Progress 26: Marit G.H. van Weering. Towards a new treatment for chronic low back pain patients. Roessingh Research and Development, The Netherlands, May 2011, ISBN: 978-90-365-3180-1.

Progress 27: Jitske Gulmans. Crossing Boundaries: Improving Communication in cerebral palsy care. Roessingh Research and Development, The Netherlands, Febuary 2012, ISBN: 978-90365-3305-8.

Progress 28: Birgit Molier. Influence of augmented feedback on learning upper extremity tasks after stroke. Roessingh Research and Development, The Netherlands, March 2012, ISBN: $978-$ 90-365-3296-9.

Progress 29: Rosemary Dubbeldam. Towards a better understanding of foot and ankle kinematics in rheumatoid arthritis. Roessingh Research and Development, The Netherlands, October 2012, ISBN: 978-90-365-3407-9.

Progress 30: Richard Evering. Ambulatory feeback at daily physical activity patterns. Roessingh Research and Development, The Netherlands, April 2013, ISBN: 978-90-365-3512-0.

Progress 31: Malhotra S. Does spasticity interfere with funktional recovery after stroke? Roessingh Research and Development, The Netherlands, November 2013, ISBN: 978-90-365-3567-0.

Progress 32: Monique Tabak. Telemedicine for patients with COPD. New treatment approaches to improve daily activity behaviour. Roessingh Research and Development, The Netherlands, February 2014, ISBN: 978-94-6108-590-0.

Progress 33: Hester Trompetter. ACT with pain. Measurement, efficacy and mechanisms of Acceptance \& Commitment Therapy. Roessingh Research and Development, The Netherlands, September 2014, ISBN: 978-90-365-3708-7.

Progress 34: Harm op den Akker. Smart Tailoring of Real-Time Physical Activity Coaching Systems. Roessingh Research and Development, The Netherlands, October 2014, ISBN: 97890-365-3762-9. 
Progress 35: Stephanie Jansen-Kosterink. The added value of telemedicine for physical rehabilitation. Roessingh Research and Development, The Netherlands, December 2014, ISBN: 978-90-823196-0-6.

Progress 36: Inge-Marie Velstra. Advanced insights in upper limb function of individuals with cervical spinal cord injury. Roessingh Research and Development, The Netherlands, October 2015, ISBN: 978-90-365-3929-6. 


